AperTO - Archivio Istituzionale Open Access dell'Università di Torino

Transcriptional remodeling in primary hippocampal astrocytes from an alzheimer's disease mouse model

This is a pre print version of the following article:

Original Citation:

Availability:

This version is available http://hdl.handle.net/2318/1784701

since 2021-04-10T00:34:50Z

Published version:

DOI:10.2174/1567205015666180613113924

Terms of use:

Open Access

Anyone can freely access the full text of works made available as "Open Access". Works made available under a Creative Commons license can be used according to the terms and conditions of said license. Use of all other works requires consent of the right holder (author or publisher) if not exempted from copyright protection by the applicable law. 


\section{Transcriptional remodeling in primary hippocampal astrocytes from an Alzheimer's disease mouse} model

Ruffinatti $F^{\text {a\#\# }}$, Tapella L ${ }^{\text {a\# }}$, Gregnanin I ${ }^{\mathrm{b}}$, Stevano $\mathrm{A}^{\mathrm{a}}$, Chiorino $\mathrm{G}^{\mathrm{b}}$, Canonico PL ${ }^{\mathrm{a}}$, Distasi $\mathrm{C}^{\mathrm{a}}$, Genazzani $\mathrm{AA}^{* \mathrm{a}}, \operatorname{Lim} \mathrm{D}^{* \mathrm{a}}$

Affiliations:

${ }^{\mathrm{a}}$ Department of Pharmaceutical Sciences, Università del Piemonte Orientale, Via Bovio 6, 28100, Novara,

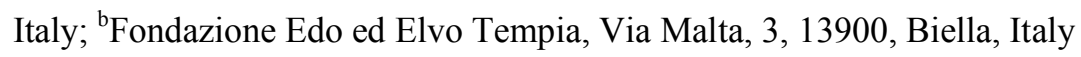

* Corresponding authors: Dmitry Lim, Department of Pharmaceutical Sciences, Università del Piemonte Orientale, Via Bovio 6, 28100, Novara, Italy. E-mail: dmitry.lim@uniupo.it; Armando Genazzani, Department of Pharmaceutical Sciences, Università del Piemonte Orientale, Via Bovio 6, 28100, Novara, Italy. E-mail: armando.genazzani@uniupo.it

\section{\# These Authors contributed equally.}

Running title: Transcriptome of cultured astrocytes from 3xTg-AD mice.

Keywords: whole-genome microarray, 3xTg-AD mice, cultured astroglia, cross-platform normalization, early Alzheimer's disease, FAD mutations. 
Abstract

Background: It is well known that alterations in astrocytes occur in Alzheimer's Disease and reactive astrogliosis is one of the hallmarks of the disease. Recently, data has emerged that suggests that alterations in astrocytes may also occur early in the pathogenesis of the disease.

Objective: The aim of present work was to characterize the transcriptional alterations occurring in cultured astrocytes from $3 \mathrm{xTg}-\mathrm{AD}$ mouse pups compared to control non-transgenic mice. Furthermore, we also compared these changes to those reported by others in astrocytes from symptomatic AD mice.

Method: We conducted a whole-genome microarray study on primary cultured astrocytes from the hippocampus of 3xTg-AD and non-transgenic mouse newborn pups. We used cross-platform normalization and an unsupervised hierarchical clustering algorithm to compare our results with other datasets of cultured or freshly isolated astrocytes, including those isolated from plaque-stage APPswe/PS1dE9 AD mice.

Results: We found a set of 993 genes differentially expressed in 3xTg-AD as compared with non-Tg astrocytes. Over-represented gene ontology terms were related to calcium, cell-cell communication, mitochondria, transcription, nucleotide binding and phosphorylation. Of note, no genes related to inflammation were found in cultured $3 \times \mathrm{Tg}-\mathrm{AD}$ astrocytes. Comparison with astrocytes isolated from plaque stage APPswe/PS1dE9 showed that 882 out of 993 genes were selectively changed in primary 3xTg-AD astrocytes while 50 genes were co-regulated and 61 were anti-regulated (regulated in the opposite direction in the datasets).

Conclusion: Our data show that in cultured astrocytes from an $\mathrm{AD}$ mouse model, transcriptional changes occur and are different from those reported in models mimicking later stages of the disease. 
1

2

3

4

5

6

7

8

9

\section{INTRODUCTION}

Alzheimer's disease (AD) is the most common dementia in the elderly. AD begins with a loss of episodic memory and progresses to cognitive decline and dementia. At the cellular level, AD is characterized by dysfunction of synaptic connectivity and neuronal death [1] and it is established that reactive astrogliosis accompanies the late stages of $\mathrm{AD}[2]$.

Astrocytes are a heterogeneous group of cells in the nervous system responsible for housekeeping and homeostatic functions. Implicated in pathogenesis of many neurodegenerative diseases [3], astrocytes may be important determinants of synaptic dysfunction in incipient $\mathrm{AD}[4 ; 5]$. For example, recent investigations on mice bearing mutations associated with familial forms of $\mathrm{AD}$ (FAD) have demonstrated that, during the disease, astrocytes do not merely become activated, but undergo complex biphasic changes. Thus in $3 x T g-$ AD mice, at three months of age astrocytes are found to be atrophic, but, after the burden of amyloid- $\beta$ (A $\beta$ ) deposits, turn to hypertrophic in the areas proximal to amyloid plaques $[5 ; 6]$.

Transcriptome studies on whole-brain tissues and on fluorescence-activated cell sorting (FACS)-isolated astrocytes from human $\mathrm{AD}$ postmortem brains as well as from a symptomatic AD mouse model have shown that at the late $\mathrm{AD}$ stages astrocytes exhibit strong inflammatory phenotypes concomitantly with $\mathrm{A} \beta$ plaque development [7]. However, much less is known about gene expression alterations and astroglial remodeling in pre-symptomatic AD. Previously, we have shown that, in primary astroglial cultures, A $\beta$ deregulates the expression of genes involved in $\mathrm{Ca}^{2+}$ signaling through the activation of $\mathrm{Ca}^{2+}$-dependent gene transcription which determines increased response to a group I glutamate receptor agonist DHPG [8-10]. Deregulation of $\mathrm{Ca}^{2+}$ signaling has been found also in astrocytes from the hippocampus of $3 \mathrm{xTg}-\mathrm{AD}$ mice, in which ATP, but not DHPG-induced $\mathrm{Ca}^{2+}$ signals were potentiated in a region-specific manner [11].

In the present study we used primary cultures of purified hippocampal astrocytes from 3xTg-AD mouse pups to explore the effects of FAD-related mutations on gene expression. Using whole-genome microarray and gene ontology (GO) analysis we show that FAD mutations alter expression of a distinct set of genes related to calcium, phosphorylation, mitochondria, gene expression and cell-cell communication. 


\section{MATERIALS AND METHODS}

\subsection{Animal handling}

The 3xTg-AD mice were obtained from Alexej Verkhratsky (Manchester University, UK; [12], 3xTg-AD mice develop both senile plaques and neurofibrillary tangles [12]). 3xTg-AD and non-transgenic (non-Tg) control mice were housed in the animal facility of the Università del Piemonte Orientale, were kept at three to four per cage, and had unlimited access to water and food. Animals were managed in accordance with European directive 2010/63/UE and with Italian law D.1. 26/2014. The procedures were approved by the local animal-health and ethical committee (Università del Piemonte Orientale) and were authorized by the national authority (Istituto Superiore di Sanità; authorization number N. 22/2013). All efforts were made to reduce the number of animals by following the $3 \mathrm{R}$ 's rule.

\subsection{Astroglial cultures}

Primary astroglial cultures were from postnatal day 0-3 (P0-P3) non-Tg and 3xTg-AD mouse pups. Hippocampi were dissected from brains, washed twice in cold HBSS (Sigma, Cat. H6648), minced with a scalpel and incubated in 1x Trypsin (Sigma) for $25 \mathrm{~min}$ ( $5 \mathrm{ml}$ of Trypsin for each 6-8 pups). After neutralization with complete culture medium (DMEM (Sigma, Cat. D5671) supplemented with $2 \mathrm{mM}$ glutamine and $1 \%$ penicillin/streptomycin (P/S, both from Sigma) ), the tissues were centrifuged ( $250 \mathrm{xg} 3$ min) and resuspended in HBSS supplemented with $10 \%$ fetal bovine serum (FBS, Gibco, Cat. 10270), 20 $\mathrm{u} / \mathrm{ml}$ DNAse and $\mathrm{MgSO}_{4}$. Hippocampal tissues were disaggregated with 30 strokes of $1000 \mu 1$ automatic pipette. $5 \mathrm{ml}$ of HBSS were added and tissue residues were allowed to sediment. Cellular suspension was transferred in a fresh falcon and, after centrifugation ( $200 \mathrm{xg}, 5 \mathrm{~min})$, pellet was resuspended in complete culture medium and cells were plated in $25 \mathrm{~mm}^{2}$ flasks (Falcon, Corning, USA). When confluent, astrocytes were detached with trypsin and contaminating microglial cells were eliminated by negative selection using anti-CD11b-conjugated magnetic-activated cell sorting (MACS) beads (Miltenyi Biotech, Cat. 130-093634). MACS was performed according to manufacturer's instructions. At the end of the separation purified astrocytes were resuspended in complete culture medium and plated for experiments. Immunocytochemical examination using anti-GFAP and anti-Iba1 antibody showed absence of microglial cells present in cultures.

\subsection{RNA isolation and whole-genome microarray}

Gene expression profiling was evaluated from 5 samples from non-Tg control mice and 5 samples from $3 \times T g-A D$ mice. For each sample $0.5 \times 10^{6}$ of MACS-purified astrocytes were plated in $65 \mathrm{~mm}$ Petri dishes in $4 \mathrm{ml}$ of complete culture medium. At 80\% confluence the medium was changed to $4 \mathrm{ml} \mathrm{DMEM/F12} \mathrm{(Gibco,}$ Cat. 21041-025, supplemented with $2 \mathrm{mM}$ glutamine and 1\% P/S). After 24 hours astrocytes were washed with PBS and lysed in $700 \mu \mathrm{l}$ QIAzole (Qiagen, Milan, Italy). Total RNA was extracted using Absolutely RNA miRNA kit (Agilent) according to manufacturer's instructions. The samples were subject to one-color gene expression profiling with SurePrint G3 mouse GE 8x60K microarrays (Agilent Technologies, Santa 
Clara, CA). Slides were scanned with an Agilent $\mathrm{C}$ dual-laser microarray scanner and images analyzed with Agilent Feature Extraction software. Raw data were normalized through the quantile between-array normalization algorithm and the empirical Bayes method (LIMMA/Bioconductor package) was used to compute a moderated $t$-statistics for two-class comparison analysis [13]. $P$-values were adjusted for multiple testing by using Benjamini-Hochberg (BH) false discovery rate (FDR) correction [14]. Because of the high degree of correlation between the genewise variance and the average expression $(\rho=0.9)$, no intensity filtering procedure was applied to not alter the variance distribution. On the contrary, LIMMA was applied to the whole unfiltered dataset to preserve full statistical power and then the expression values of the genes called as significantly regulated have been checked a posteriori [15]. Functional annotation was performed using the DAVID Bioinformatics Resources v. 6.7 (https://david.ncifcrf.gov/) [16]. False discovery rate (FDR) correction was applied to overrepresented GO terms and cutoff was set to $p<0.1(10 \%)$.

\subsection{Real-time PCR}

Validation of the microarray results by real-time PCR was performed on 4 non-Tg and 4 3xTg-AD astroglial cultures. Total RNA extraction was performed as described above. 0.5-1 $\mu$ g of total RNA was retrotranscribed using random hexamers and ImProm-II RT system (Promega, Milan, Italy). Real-time PCR was performed using iTaq qPCR master mix according to manufacturer's instructions (Bio-Rad, Segrate, Italy) on a SFX96 Real-time system (Bio-Rad). To normalize raw real-time PCR data, three housekeeping genes were tested: i) $\beta$-actin (Actin), ii) S18 ribosomal subunit and iii) polymerase (RNA) II (DNA directed) polypeptide A (RP2a). S18 showed highest stability across the samples and was used for normalization of raw data in all real-time PCR experiments. Sequences of oligonucleotide primers are provided in Supplementary Materials. The real-time PCR data are expressed as delta-C(t) of gene of interest to S18 allowing appreciation of the expression level of a single gene.

\subsection{Comparison with the transcriptome database of acutely isolated purified CNS cellular models}

To assess the relationship existing among our astrocyte cultures and the samples belonging to the transcriptome framework of acutely isolated purified central nervous system (CNS) cell types as provided by Cahoy et al. [17], GSE9566 series was downloaded from Gene Expression Omnibus (GEO) database (https://www.ncbi.nlm.nih.gov/geo/). It consisted in a transcriptome database made up of 48 independent murine samples measuring the expression levels of more than 20,000 genes. Three main CNS cell types, astrocytes, neurons and oligodendrocytes, are profiled at various postnatal ages, allowing for the identification of those enrichment patterns specifically characterizing each cell type. In particular, for this study, Affymetrix Mouse Genome 4302.0 Array platform was used, featuring 45,037 oligonucleotide probes (representing 20,832 unique genes) per array. Text matrix file available from GEO database contained $\log _{2}$ expression values that were already between-array normalized. In order to relate our expression data with those provided by Cahoy and colleagues an "early integration" approach was needed which allowed the data to merge before analysis [18]. To this purpose, the cross-platform normalization (XPN) algorithm was used 
as described by Shabalin et al. in [19] to produce a single unified dataset. More in detail, Ensembl BioMart on-line application (https://www.ensembl.org) was used to match probes from different platforms (namely Agilent and Affymetrix) and just those 20,932 probes (12,416 unique genes) identified by Cahoy as significantly and consistently expressed in at least one CNS cell type (Supplemental Table S3 in [17]) were used to assembly the common probe-set. This procedure led to a final list of 18,135 cross-platform corresponding probes, representing 11,589 different genes (93.3\% of Cahoy's filtering selection). Moreover, only the samples corresponding to one of the groups of interest were retained for XPN procedure and downstream comparison with our dataset, namely acutely isolated $\mathrm{P} 17$ astrocytes $(\mathrm{n}=5)$, cultured astroglia $(n=4)$ and acutely isolated P16 neurons $(n=3)$. Prior to XPN merging, expression values from each platform were column standardized (zero mean and unitary standard deviation). To reliably evaluate the distance of our samples compared to those from Cahoys's dataset - that includes many different experimental groupsnon- $\mathrm{Tg} \log _{2}$ expression values were separately integrated into Cahoy's dataset through XPN algorithm. This precaution allowed indeed to maximally reduce batch effect and helped to rule out possible confounding factors reflecting the inner structure of the two datasets. Specifically, the common experimental conditions adopted for tissue dissociation and cell culturing were likely to put in contact our two cellular models when clustering samples, thus possibly distorting their individual relation to Cahoy's models. Unsupervised hierarchical clustering for sample positioning assessment was performed using Euclidean metric and average linkage criterion. All dendrograms presented in the text reflect this particular choice even if other different metrics would have led to analogous results from a qualitative point of view (not shown). The analysis then proceeded as described in the Results section, searching for those particular genes that were enriched in (or shared by) non-Tg astrocytes cultures compared to the cellular models of interest present in Cahoy's dataset. All these ranked lists of differentially expressed genes were produced through a two-class unpaired rank product (RP) algorithm [20], conducted on $\log _{2}$ expression values as returned by XPN procedure, controlling FDR at 0.005 level as detailed in Results section. Just for the gene list-associated heatmaps, a further gene/row-wise normalization step (log-mean subtraction) was performed in order to represent the expression values of each gene in terms of $\log _{2}$ fold-enrichment over their mean value across all samples.

\subsection{Comparison with APPswe/PS1dE9 dataset and normally aged mice}

To compare our microarray dataset with the AD dataset from the APPswe/PS1dE9 double-transgenic mouse model [7], GSE74615 series was downloaded from Gene Expression Omnibus (GEO) database (https://www.ncbi.nlm.nih.gov/geo/). It consisted in a text matrix file containing $\log _{2}$ intensity values from 22 samples of cortical microglia and astrocytes isolated from 15- to 18-month-old AD and wild-type (WT) mice. RNA samples were hybridized onto Mouse GE 4x44k v2 microarrays (Agilent). In particular, each array featured 39,429 different probes, the $1 \%$ of which (399 probes) being within-array replicates, for a total of 43,020 probes per array. Downloaded data were already normalized through quantile between-array algorithm. Thanks to the presence of an internal control (WT mice), we could address this comparison avoiding the introduction of additional confounding factors by a "late integration" (or meta-analysis) 
approach [18]. A cross-platform normalization between Orre's and our dataset was indeed not suitable being the two studies representative of two different healthy and AD pathological models. Therefore, unlike what we did for integrating our dataset into the transcriptome framework provided by Cahoy and colleagues, in this case the individual microarray studies were separately processed to obtain two lists of differentially expressed genes (ranked on the basis of their $p$-values) which have been then combined in the final step of the analysis. In particular, for both dataset, differential expression of all genes among groups was tested for significance through an empirical Bayes moderated $t$-test (LIMMA/Bioconductor package). $P$-values were adjusted for multiple comparisons by using BH FDR correction and genes featuring an adj.p.val $\leq 0.05$ were marked as significant. APPswe/PS1dE9 whole dataset was employed for the empirical Bayes test, but replicate probes were finally collapsed by mean and microglia data were discarded to retain only the contrast of interest (AD astrocytes vs. WT astrocytes) for downstream comparison with our dataset (4 samples for $\mathrm{AD}$ and 4 samples for WT; 2,578 differentially expressed unique genes out of 39.429 different probes). Sharing the same technological platform (i.e. Agilent), the gene-matching procedure between the two datasets was straightforward. All of our 993 significant genes found a correspondence into the APPswe/PS1dE9 dataset: some of them were co- or anti-regulated genes while others genes resulted significantly regulated only in our model. The analysis of the transcriptome intersections between the two datasets then proceeded as described in the Results, while its statistical background is detailed in Appendix: Common significant gene analysis. To compare the changes in cultured 3xTg-AD astrocytes with those in young-versus-old mice reported by [21], as there is no available original dataset for young mice in GEO database, a list of 926 significantly regulated genes in aged compared to young cortical astrocytes was directly used for comparison (see Supplementary Table 3 in [21]). Official gene symbols were used for gene matching and the whole array (Agilent, Mouse GE 4x44k v2 microarray) has been used as reference for the subsequent analysis. The significance of the transcriptome intersections was assessed through the same approach already mentioned and described in Appendix.

\subsection{Comparison with human AD dataset}

The human AD reference dataset used for comparing our microarray output was made available by Berchtold et al. [22] through the publicly accessible GEO database: series accession number GSE48350. This dataset consisted of 253 samples from 4 brain regions (hippocampus, entorhinal cortex, superior frontal cortex and post-central gyrus), from both normal controls and AD patients. RNA samples were hybridized onto Affymetrix Human HgU133 plus 2.0 microarrays. In particular, each array featured 54,675 different probes and no within-array replicates. Downloaded data were already between-array normalized (GC-Robust Multiarray background adjustment and quantile normalization). Also in this case, thanks to the presence of an internal control (normal non-AD subjects), we could address this comparison between different models through the same "late integration" approach [18] already used for the comparison with APPswe/PS1dE9 double-transgenic mouse dataset. Again, individual microarray studies were separately processed to identify two lists of differentially expressed genes (ranked on the base of their $p$-values) which have been then 
combined in the final step. In particular, data from Berchtold's dataset were then $\log _{2}$-transformed and differential expression of all genes among the groups of interest (69- to 99-year-aged control hippocampus, $\mathrm{n}=24$; 74- to 95-year-aged AD hippocampus, $\mathrm{n}=18$ ) was tested for significance through an empirical Bayes moderated $t$-test (LIMMA/Bioconductor package). $P$-values were adjusted for multiple comparisons by using BH FDR correction and genes with an adj.p.val $\leq 0.05$ were marked as significant. This procedure led to the identification of 746 differentially expressed genes. Ensembl BioMart on-line application (https://www.ensembl.org) was then used to find human-mouse orthologous genes and to match probes from different platforms (Agilent and Affymetrix respectively). A virtual set of 21,213 one-to-one orthologous genes was obtained (96.4\% of which featuring a "high human orthology confidence"), but just 495 out of the 993 genes that were significant into our murine dataset found an orthologous correspondence within the human dataset provided by Berchtold et al. (329 genes did not featured any human orthology while 169 were not present in Berchtold's dataset). As for the previous comparison with APPswe/PS1dE9 samples, we found co-regulated genes, anti-regulated genes and genes significantly regulated only in our model. The subsequent analysis of the transcriptome intersections between the two datasets is described in the Results section while its statistical background is detailed in the Appendix Common significant gene analysis.

\subsection{Software used for microarray analysis}

Quantile normalizations and empirical Bayes moderated $t$-tests were conducted in R (using LIMMA/Bioconductor package). XPN algorithm was run in MATLAB environment using the code as provided by the author [19]. RP procedures for gene selection and unsupervised hierarchical clustering, such as unsupervised hierarchical clustering and related dendrograms were performed using MeV 4.9.0 (Multi Experiment Viewer, TM4 Software Suite) [23]. The same software was used also to draw dendrograms and heatmaps from expression data. Common significant gene analysis was performed as detailed in Appendix through homemade code. 


\section{RESULTS}

\subsection{3 genes are differentially expressed in 3xTg-AD vs non-Tg cultured hippocampal astrocytes}

Nine independent cultures were prepared from non-Tg or 3xTg-AD mice. Before plating, astroglial cultures were depleted of microglial cells to avoid contaminations. This resulted in virtually no microglial cells detected by anti-Ibal immunocytochemical analysis (Fig. 1A). Five non-Tg and five 3xTg-AD cultures were processed for microarray analysis while the remaining cultures were used for validation using real-time PCR. A total of 55,681 Agilent mouse probes were analyzed. Based on our previous experience, we expected overall low changes in gene expression $[9 ; 10]$. We therefore did not apply any cut-off for fold change and considered all genes which passed BH FDR correction for multiple comparisons (Adjusted p-value, Adj.p.val) with $\mathrm{p} \leq 0.05$. Probes representing long intergenic non-coding RNAs (lincRNA), as well as probes with no gene symbol, no gene name and no description were excluded from the selection. Such an approach yielded 993 differentially expressed genes in 3xTg-AD vs non-Tg samples (Fig. 1B). 536 genes were upregulated and 457 genes were down-regulated. The full list of differentially expressed genes may be found in Supplementary Table 1, while the top 40 of up- and 40 down regulated genes are listed in Table 1. In total, 33 genes were used for validation by real-time PCR on 4 independent cultures. Of these, 27 genes passed validation and are listed in Supplementary Table 2. Figure 2A demonstrates the high degree of correlation between differential expression obtained in microarray and real-time PCR, while real-time PCR results of a set of 14 genes is shown in Figure 2B, demonstrating the reliability of the microarray results.

\subsection{Gene Ontology analysis does not detect inflammation but impairment of cell-cell communication and transcription.}

The possible biological significance of the differentially expressed genes was analyzed using DAVID online GO tool v. 6.7 (https://david.ncifcrf.gov/). In the up-regulated genes, the most general over-represented GO terms were phosphoprotein, cytoplasm, alternative splicing, acetylation, transferase and nucleotide binding. More specific GO terms included mitochondrion, ATP binding, cell projection, Zinc finger, C2H2-like and Krueppel-associated box, compositionally biased region:Poly-Arg and vesicle (Table 2). The down-regulated genes were specifically enriched in the following general GO terms: phosphoprotein, cytoplasm, alternative splicing, cytoplasm and transport. More specific over-represented GO terms were nucleolus, cell projection and cell junction, calcium, apoptotic process, synapse and dendritic spine, and extrinsic apoptotic signaling pathway. The most enriched GO terms among down-regulated genes were EH domain, positive regulation of $T$ cell mediated cytotoxicity, triglyceride catabolic process and extrinsic apoptotic signaling pathway (Table 2). Previously, we reported that astroglial $\mathrm{Ca}^{2+}$ signaling is deregulated in $\mathrm{A} \beta$-treated astrocytes [8-10] as well as in astrocytes from 3xTg-AD mice [11]. Therefore $\mathrm{Ca}^{2+}$-related genes were of special interest. However, overrepresented $\mathrm{Ca}^{2+}$-related genes in down-regulated hits did not contain genes of the classical " $\mathrm{Ca}^{2+}$ signaling toolkit" but almost all coded for $\mathrm{Ca}^{2+}$-regulated proteins involved in cell adhesion, exocytosis but mostly in formation of extracellular matrix (Table 3). Validation of these genes by real-time PCR is 
shown in Figure 2. Taken together, GO analysis suggests that $\mathrm{Ca}^{2+}$-regulated cell adhesion and cell-cell communication, mitochondria and transcription may be specifically deregulated in 3xTg-AD cultured astrocytes.

\subsection{Comparison with cultured and freshly FACS-isolated astrocytes.}

To our knowledge, there are no published reports on the transcriptional profile of cultured astrocytes from the hippocampus. Therefore, it was interesting to compare the transcriptional profile of our cultured non-Tg astrocytes with that of cultured cortical astrocytes, freshly FACS-isolated forebrain astrocytes P17 pups, or freshly isolated neurons from P16 pups provided by Cahoy and colleagues [17]. We used the Ensembl BioMart on-line application (https://www.ensembl.org) for matching genes between different platforms, namely Agilent Mouse GE 4x44k v2 and Affymetrix Mouse Genome 430 2.0 Arrays. This procedure led to a set of 18,135 common probes, where only those probes filtered by Cahoy as corresponding to "genes with significant expression in the CNS" (Supplemental Table S3 in [17]) were taken into account. After the two datasets were properly integrated and renormalized through XPN algorithm (see Materials and Methods), they were merged into one single dataset. Since XPN algorithm succeeded in removing the batch effect without altering the original clusterization among samples from Cahoy's dataset (not shown), we could proceed performing an unsupervised hierarchical clustering (Euclidean distance metric and average linkage criterion) to inspect the positioning of our non-Tg astrocytes compared to the groups profiled by Cahoy et al. Figure $3 \mathrm{~A}$ shows that our cultured astrocytes resulted more similar to the cultured and freshly isolated astroglia than in respect to any other cell type in Cahoy's dataset. Secondly, both cultured cellular models were more similar to acutely isolated astrocytes than to neurons. Incidentally, the fact that our samples could penetrate Cahoy's dataset, clustering with its cultured astroglia, is a good evidence that the batch effect was efficiently removed. Afterwards, we took advantage of the merged dataset to search for the most up- and down-regulated genes in our non-Tg astrocyte cultures compared to acutely isolated astrocytes. We used the rank product method (two class, unpaired) as described in [20] controlling FDR at the strict level of 0.005 to get a basic ranked list of genes representing the most enriched expression patterns in both the groups. Results are shown in Figure 3B,C respectively. In particular, we found 130 up-regulated genes in non-Tg compared to acutely isolated astrocytes (Supplementary Table 3) and 230 down-regulated genes in non-Tg compared to acutely isolated astrocytes (Supplementary Table 4). Notably, even if the statistical test was conducted only by comparing non-Tg against acutely isolated astrocytes, heatmaps clearly showed that Cahoy's cultured astrocytes closely followed our non-Tg culture expression profiles. This is another evidence of the high degree of similarity between our cultured model and Cahoy's, restricted in particular to those genes that are the most specific for cultured astrocytes. More in detail, $70.0 \%$ of genes enriched in our non-Tg cultures could also be found in Cahoy's list "Genes statistically enriched in cultured astroglia compared to in vivo astrocytes" (Supplemental Table S21 in [17]), while 76.5\% of genes down-regulated in non-Tg ould also be found in Cahoy's list "Genes statistically enriched in in vivo astrocytes compared to cultured astroglia" (Supplemental Table S20 in [17]). Taken together, these two percentages give an estimate of the technical 
reproducibility and the biological variability of in vitro astrocyte cell cultures. On the other hand, a complementary list of the most up- and down-regulated genes in our non-Tg cultures compared to Cahoy's cultured astroglia (two class unpaired rank product, $\mathrm{FDR}=0.005$ ) is provided as supplementary material (Supplementary Tables 5 and 6). As a final qualitative remark, the expression profile of acutely isolated neurons (right side of the heatmaps, Figure 3B,C) also shows how most of the genes that are differentially expressed in non-Tg cultures compared to acutely isolated astrocytes can be indeed ascribable to a neuronal phenotype.

\subsection{Comparison with isolated astrocytes from symptomatic $\mathrm{AD}$ or normally aged mice}

Notably, in cultures from 3xTg-AD, genes involved in immune responses were largely unchanged. This is in sharp contrast with changes occurring in aged plaque stage (15 mo of age) APPswe/PS1dE9 AD mice [7] in which the most prominent change was the up-regulation of a large list of genes related to different aspects of inflammation. We therefore compared our genes with the publicly available dataset by [7] (GEO database accession number: GSE74615). To this purpose, APPswe/PS1dE9 dataset was downloaded and the empirical Bayes method (LIMMA) was used to assess the significance of differential expression of all genes among groups. After $p$-values were adjusted through BH procedure, genes with an adj.p.val $\leq 0.05$ were marked as significant. While the original APPswe/PS1dE9 dataset included both cortical astrocyte and microglia samples, only the astrocyte data were retained for use in the following meta-analysis (see Materials and Methods).

Each one of the 993 genes we found to be significantly regulated in our model had a direct correspondence within Orre's dataset. In particular the comparison between 3xTg-AD significant genes and APPswe/PS1dE9 dataset allowed to define 6 distinct categories of genes corresponding to 3 relationships between the datasets (see heatmaps and table in Figure 5 for a graphical summary): 1) significantly co-regulated genes (total 50, upward 23, downward 27); 2) significantly changed only in 3xTg-AD astrocytes (total 882; up-regulated 486, down-regulated 396); and 3) genes oppositely regulated in 3xTg-AD and APPswePS1dE9 astrocytes (total 61, up-to-down 27, down-to-up 34). Notably, this kind of analysis could have been alternatively addressed through a K-means algorithm, but in that case the resulting 6 clusters would have been based merely on $\log _{2} \mathrm{FC}$ expression values, and all the information related to the $p$-values of the second dataset (APPswe/PS1dE9) would have been ignored. Conversely, to find the common and the specific gene signature of the two pathological models compared, we preferred clustering genes using the more rigorous criterion of the statistical significance compared their own whole dataset. Although a number of methods for the meta-analysis of combined lists of $p$-values from two or more studies exist [18; 24], some of them (e.g. Fisher's, Stouffer's, minP) are too liberal for our purpose - since genes having small $p$-values in just one study are likely to be detected as globally significant—while others (such as maxP) are conservative but too naïve, not addressing the problem of distinguishing concordant and discordant expression fold changes [18], nor the problem of randomly shared genes. In fact, it should be considered that when two datasets are compared in terms of shared significant genes, a certain number of co-/anti-regulated genes is expected to 
arise just by chance. For this reason, here we propose a novel method for the meta-analysis of $p$-valued lists of genes drawn from two different studies. The procedure makes use of a simple randomized null model based on the binomial distribution to determine which (if any) of the 6 relational categories of genes was significantly enriched (see Common significant gene analysis in Appendix). Overall, this method is useful to compare differentially regulated genes between two studies in order to find their " $\log _{2}$ FC-signed" intersections and their statistical significance according to quite conservative criteria.

With reference to the table in Figure 4, the central column contains all genes we found significantly changed only in 3xTg-AD astrocytes (Up-Zero and Down-Zero). All these 882 genes can be considered, as a whole, specific for cultured 3xTg-AD astrocytes, possibly representing early FAD-related alterations. They can also be used for further analysis (such as biological validation through GO analysis, see below) regardless of their categorical $p$-value. However, the smaller the $p$-value, the more likely that the two AD models compared have a substantial overlap, because the number of significant non-shared genes is too low if compared to the null model. In other words, the number of common genes differentially expressed is too high compared to a random expression pattern, such as that resulting from comparing two completely independent disease models. In our case the very low $p$-value assigned to the category of genes significantly downregulated only in 3xTg-AD suggests that at least one of the two other categories of co-regulated and anti-regulated genes (left and right column respectively) were enriched. This allowed us to search for those processes that are in common for both models of early and late AD. In particular, we found 27 common genes significantly downregulated in both the dataset (Down-Down, expected value $=14.9$; categorical $p$-value $=0.003$ ) and 34 genes significantly down-regulated in 3xTg-AD and significantly up-regulated in APPswe/PS1dE9 astrocytes (Down-Up, expected value $=22.6$; categorical $p$-value $=0.013$ ).

To analyze the biological significance of genes specific for primary astrocytes from 3xTgAD mice we first fed to DAVID GO tool a list of 882 genes that that were not changed in APPswe/PS1dE9 astrocytes (Supplementary Table 7) regardless they were up or down-regulated. These genes constitute the major part $(89 \%)$ of all differentially expressed genes of our dataset, therefore, expectedly, enriched GO terms (Supplementary Table 8) overlapped significantly with those found during the initial analysis (Table 2). This suggests that in the $3 \times \mathrm{Tg}-\mathrm{AD}$ cultured astrocytes the altered astroglial functions differ substantially from those altered later on, when deposition of $A \beta$ aggregates is fully expressed. These alterations comprise genes and functions described by GO terms phosphoproteins, cytoplasm, alternative splicing, nucleotide and ATP binding, transferase activity, mitochondrion, cell projection, apoptotic process, magnesium, Kruppel associated box and $K R A B$.

Next, we analyzed GO significance of two small categories (co-regulated and anti-regulated) in which the number of common genes significantly differed from expected values (Figure 5). In the list of co-downregulated genes (Down-Down, Figure 5) GO analysis failed to find significantly enriched GO terms. However, in the list of 34 anti-regulated genes (down 3xTg-AD and up in APPswe/PS1dE9 astrocytes, Down-Up) there were 5 GO terms with significantly enriched genes: membrane, nucleolus, ribosomal 


\subsection{Comparison with human AD microarray}

A this point it was interesting to explore if the changes found in cultured 3xTg-AD astrocytes may have had common/divergent features with the changes observed in postmortem biopsies from symptomatic $\mathrm{AD}$ patients. The hypothesis was that this was unlikely to occur, as cultures possibly represent early changes. For this, we downloaded from GEO database the dataset contributed by Berchtold et al. [22] (series accession number: GSE48350). This dataset featured samples from normal controls (young and aged) and AD cases, from 4 brain regions (hippocampus, entorhinal cortex, superior frontal cortex, post-central gyrus). After the assessment of the differential expression (LIMMA/empirical Bayes) and $p$-values adjustment for multiple comparisons (BH procedure), genes with an adj.p.val $\leq 0.05$ were marked as significant. Then we restricted our analysis to the groups of interest, retaining just hippocampal samples from both aged control subjects $(\mathrm{n}=24)$ and aged AD patients $(\mathrm{n}=18)$. During the gene-matching procedure (see Materials and Methods for details) we searched for those genes that met the following requirements: i) belonged to the subset of the genes significantly regulated in our mouse dataset; ii) featured at least one human orthologous gene iii) were present (as orthologous gene) within the Berchtold's human dataset. Because of these necessary prerequisites, the pool of significant genes available for comparison with the human dataset decreased from 993 to 495 . 
Starting from this set of selected genes, the analysis proceeded according to the same methods already described in the previous section and following the same statistical approach. Contrary to what emerged from the comparison with the APPswe/PS1dE9 model, in this case we were not able to find any particular type of enrichment in any of the 6 relational categories previously described. In other words, the empirical configuration of the common significant genes was precisely predicted by the null model, thus suggesting a substantial independence of the two datasets (Supplementary Table 10). While, on the one hand, this negative result can be an indication of the large number of different biological parameters distinguishing the two models compared (mouse vs. human, astrocytes vs. whole-tissue samples, young vs. aged, familial AD vs. sporadic $\mathrm{AD}$ ), it may also confirm the transcriptional specificity of early astroglial changes compared to the late astroglial changes. Moreover, the near-perfect agreement between empirical and expected values demonstrates the high predictive power of our null model in the case of uncorrelated datasets, and hence the substantial validity of this statistical approach.

\section{DISCUSSION}

In the present report we have investigated transcriptional alterations in purified cultures of hippocampal astrocytes of $3 \times \mathrm{Tg}-\mathrm{AD}$ mice. An obvious limitation of this work is the use of astrocytes in culture, which raises concerns on (i) to which extent the changes in gene expression in cultures reflect the changes occurring in astrocytes in vivo, and (ii) to which extent the cultured astrocytes from FAD mice models represent $\mathrm{AD}$. With regard to the first point, while it has been reported that in defined growing conditions gene profiles of cultured astrocytes in different aspects reliably replicate those of freshly isolated astrocytes ([25] and refs therein), our first objective was not to replicate gene expression of astrocytes in vivo, but to find the early FAD-related transcriptional signature specific to astrocytes in the hippocampal region as compared with non-Tg astrocytes. With this aim the purity of astrocytes in culture was our first concern because small contaminations of astrocytes with microglial cells would result in the emergence of transcripts related to inflammation. This was successfully achieved by eliminating microglia with anti-CD11b beads. Regarding the second point, we reasoned that astroglial cultures prepared from newborn mouse pups may represent a proxy model for early, pre-plaque, $\mathrm{AD}$ because the changes (if any) would have been due to the FAD-related mutations but not due to the burden of $A \beta$ species. Of the three mutations present in $3 \times T g-A D$ mice, $P S 1_{\mathrm{M} 146 \mathrm{~V}}$ mutation has been introduced by knock-in of a point mutation of in the PS1 gene and is ubiquitous as it is under the control of the endogenous PS1 promoter [26]. The other mutations, APPswe and $\mathrm{Tau}_{\mathrm{P} 301 \mathrm{~L}}$ have been added later as overexpressing human transgenes under the control of Thy-1.2 promoter, which is considered to be neuronal [12]. Therefore, in vivo the effect of the PS1 $1_{\mathrm{M} 146 \mathrm{~V}}$ mutation should have direct consequences on astroglial biology, while the effects of APP and Tau mutations should be indirect. In cultured astrocytes, the changes (if any) should be a consequence of PS1 mutation, while the effects of APP and Tau mutations appear to be less probable or indirect, driven by priming before explant. 
In contrast to works reporting proinflammatory phenotypes in cultured astrocytes from FAD mouse models [27] as well as in A $\beta$-exposed astrocytes [28], in our highly purified astroglial cultures from 3xTg-AD hippocampi we found substantially no changes in inflammatory genes with down-regulation of a few genes related to positive regulation of $\mathrm{T}$ cell mediated cytotoxicity. Recently, however, we have found that transforming growth factor $\beta$ (TGF- $\beta$ ) is released by cultured hippocampal astrocytes [29]. Now this observation is corroborated by the finding that TGF- $\beta 2$ and TGF- $\beta 3$ are expressed at high levels in hippocampal astrocytes and TGF- $\beta 3$ is significantly up-regulated in $3 \times \mathrm{Tg}-\mathrm{AD}$ with regard to non-Tg astrocytes.

Among highly up-regulated genes in 3xTg-Ad astrocytes the only gene relative to the immune system was C-C motif chemokine ligand $27 \mathrm{a}(\mathrm{Ccl} 27 \mathrm{a})$, a member of the $\beta$-chemokine family of proinflammatory mediators [30]. However, a closer examination of the 3 probes in the Agilent array for this cytokine, all of which were up-regulated, shows that two probes detected a splice variant of $\mathrm{Ccl} 27 \mathrm{a}$ in which the signal peptide was replaced with an alternative stretch of amino acids that allows for nuclear targeting of this isoform. This nucleus-targeted Ccl27a variant, which is called PESKY [30] is the most expressed isoform in primary astrocytes and is able to modulate gene transcription [31]. This finding corroborates the hypothesis that transcriptional reprogramming occurs in $3 \mathrm{xTg}-\mathrm{AD}$ astrocytes as well as the absence of inflammation. Our results suggest that hippocampal astrocytes from a mouse FAD model are unable to mount a proinflammatory phenotype in a cell-autonomous manner. The setup of inflammation likely requires participation of microglial cells which, together with astrocytes, upon exposure to activating stimuli, like $\mathrm{A} \beta$, undergo cascading reactions which result in activation of both microglial and astroglial cells [32]. Such a scenario may be illustrated by a recent report by Balducci et al. [33] in which acute in vivo intraventricular delivery of oligomeric $A \beta$ induced rapid and long-lasting inflammatory reactions with astroglial expression of pro-inflammatory cytokines. Such an observation also highlights that the strength of our model, i.e. purified astrocytes, on the other hand limits the possibility to evaluate the relationship between cell types in early $\mathrm{AD}$. In this regard it is interesting to note that in late stages AD pathology patients with APOE 4 allele astrocytes exhibited alterations in genes related to cell adhesion and signaling pathways including calcium signaling and insulin signaling, while changes in expression of inflammatory genes were not reported [34]. Previously, we have suggested that astroglial degeneration, occurring in the very early AD stages, may be linked to deregulated astroglial $\mathrm{Ca}^{2+}$ signaling [5; 35]. Reasonably, astroglial degeneration is likely to be associated with the absence or the repression of inflammatory processes, while it would be hardly reconcilable with inflammation. Instead, at later AD stages, in which the inflammatory phenotype is fully expressed [7], astrocytes are found to be reactive and hypertrophic [2].

GO analysis of our dataset suggests that physiological processes and functions which are likely to be altered in 3xTg-AD astrocytes are: phosphorylation, transcription, mitochondrial structure and function, calciumregulated cell adhesion and cell-cell communication. A survey of the literature suggests that alterations in all these functions occur in symptomatic $\mathrm{AD}[36 ; 37]$. Considering astroglial cultures from new-born pups a 
proxy model for the early developing pre-plaque $\mathrm{AD}$, our analysis suggests that phosphorylation, mitochondria, cell adhesion and cell-cell communication are among the first to be altered in astrocytes. In particular, alterations in phosphorylation cascades and in autophagy which are controlled by mammalian target of rapamycin complex 1 (mTORC1) are emerging as possible causes of metabolic dysregulation in neurodegenerative diseases including $\mathrm{AD}[36 ; 38 ; 39]$. In this regard, of note is one of the most downregulated genes in 3xTg-AD astrocytes, Sterile alpha motif domain containing protein 4 (Samd4). Samd4 is a mammalian homolog of Drosophila Smaug gene coding for a protein able to bind RNA and repress translation [40]. While the function of Samd4 in mammalian cells, including astrocytes, is largely unknown, it has recently been reported that mice with missense mutation of Samd4 were exceptionally thin and had severe deficiency in metabolic homeostasis and mTORC1 signaling [41]. Our finding that a protein required for the mTORC1 signaling is strongly downregulated in FAD-expressing astrocytes suggests that such a deregulation may initiate early in $\mathrm{AD}$ pathogenesis.

One important feature of late $\mathrm{AD}$, as it has been shown on FACS-isolated astrocytes, is the down-regulation of genes involved in cellular communication and neuronal support [7]. In our dataset, in contrast to genes coding for inflammatory mediators, we found that many gene which code for proteins involved in exocytosis, formation of processes and extracellular matrix, which generally may be involved in communication with surrounding cells and support to neurons, are massively down-regulated in 3xTg-AD astrocytes. This suggests that the impairment of communication between astrocytes and other cells in the brain may begin very early in AD. In line with this observation, Stenovec et al [42] reported impaired vesicle dynamics and reduced evoked secretion of a neuroactive peptide atrial natriuretic peptide in $3 \times \mathrm{Tg}-\mathrm{AD}$ cultured astrocytes. These alterations were attributed to mutations in PS1. The impairment of cell-cell communication, in concomitance with compromised housekeeping functions may be at the basis of early astrogliopathy that, in turn, accelerates the impairment in synaptic transmission [43].

Comparison of the dataset presented in this work with the dataset obtained from astrocytes isolated from plaque-stage symptomatic APPswe/PS1dE9 AD mice [7] shows that most altered genes (89\%) were specific for cultured astrocytes and were not changed in APPswe/PS1dE9 mice. This suggests that (1) in cultured 3xTg-AD astrocytes as a proxy for early $\mathrm{AD}, \mathrm{FAD}$-mutations produce a unique signature of transcriptional remodeling which may be related to changes occurring early in $\mathrm{AD}$; and (2) dynamics of astroglial remodeling are likely to change significantly during $\mathrm{AD}$ progression. Alongside the $\mathrm{AD}$-related changes, astrocytes are involved in ageing. Transcriptional changes in $\mathrm{AD}$, however, differ from those related to ageing by exacerbated inflammatory response and increased dysfunction [7;21]. Our attempt to compare alterations in cultured $3 \times \mathrm{Tg}-\mathrm{AD}$ astrocytes with the aged-vs-young changes in mice suggests that FADmutations in cultured astrocytes produce changes which are more related to AD pathology than to normal ageing. Considering that in different brain areas the dynamics of AD-related pathology are brain regionspecific [5;11], it is interesting to note that in humans, astrocytic genes, which are mostly expressed during ageing tend to lose their region specificity undergoing global up-regulation [44]. 
While in AD neuropathological changes are not always followed by cognitive deficit, and cognitively normal subjects may have deposition of amyloid plaques and development of neurofibrillary tangles, it appears important to correlate cognitive damage with astroglial gene expression profile. Such an attempt has been recently made by Barbash and colleagues [45]. In contrast to neuronal and oligodendrocytic genes, which were up-regulated and down-regulated, respectively, in non-demented patients with AD neuropathology, astroglial and microglial genes were up-regulated in both demented and non-demented AD patients as compared with healthy subjects. These results may be interpreted as there is no correlation in astroglial overall transcriptome changes and cognitive damage, but only with neuropathology [45]. However, more detailed examination of functional significance of changed genes is necessary for further understanding of their relation cognitive damage in $\mathrm{AD}$. In this report we analyze the alterations in primary astroglial cultures of FAD-expressing mouse pups, which likely reflect the early stages of AD and may determine or even delay development of both neuropathological and cognitive alterations.

\section{Conclusion}

Astrocytes, which are the principal housekeeping and homeostatic cells in the brain, are intimately involved in the pathogenesis of neurodegenerative diseases including AD. The spatio-temporal pattern of astroglial alterations in $\mathrm{AD}$ is likely to be complex and in the early, pre-symptomatic disease stages include astrodegeneration and atrophy [5]. In this work we used cultured hippocampal astrocytes from 3xTg-AD mice as a proxy model of early astrocyte involvement in FAD to investigate transcriptional alterations that may characterize early AD using whole-genome microarray technology and subsequent bioinformatics approaches. Our results indicate that, in astrocytes, FAD mutations produce a pattern of alterations which is distinct from that reported in astrocytes at later stages, although a number of genes appear to be common in the two mouse models. Our data reinforce and provide new details to the hypothesis of the early involvement of astrocytes in AD pathogenesis and emphasize the importance of its further investigation. 


\section{Acknowledgements}

This work had the following financial supports: grants 2013-0795 to AAG and 2014-1094 to DL from the Fondazione Cariplo; grant 2015 to CD and 2016 to DL from The Università del Piemonte Orientale; and grant PRIN-2015N4FKJ4 to PLC from the Italian Ministry of Education.

\section{Conflict of Interest Statement}

The authors declare no conflict of interests. 


\section{References}

[1] Spires-Jones TL, Hyman BT. The intersection of amyloid beta and tau at synapses in Alzheimer's disease. Neuron 82: 756-71.(2014).

[2] Ben Haim L, Carrillo-de Sauvage M-A, Ceyzériat K, Escartin C. Elusive roles for reactive astrocytes in neurodegenerative diseases. Frontiers in Cellular Neuroscience 9: 278.(2015).

[3] Ferrer I. Diversity of astroglial responses across human neurodegenerative disorders and brain aging. Brain Pathology (Zurich, Switzerland) 27: 645-74.(2017).

[4] Verkhratsky A, Zorec R, Rodríguez JJ, Parpura V. Astroglia dynamics in ageing and Alzheimer's disease. Current Opinion in Pharmacology 26: 74-79.(2016).

[5] Lim D, Rodríguez-Arellano JJ, Parpura V, Zorec R, Zeidán-Chuliá F, Genazzani AA, et al. Calcium signalling toolkits in astrocytes and spatio-temporal progression of Alzheimer's disease. Current Alzheimer Research 13: 359-69.(2016).

[6] Yeh C-Y, Vadhwana B, Verkhratsky A, Rodríguez JJ. Early astrocytic atrophy in the entorhinal cortex of a triple transgenic animal model of Alzheimer's disease. ASN neuro 3: 271-79.(2011).

[7] Orre M, Kamphuis W, Osborn LM, Jansen AHP, Kooijman L, Bossers K, et al. Isolation of glia from Alzheimer's mice reveals inflammation and dysfunction. Neurobiology of Aging 35: 274660.(2014).

[8] Grolla AA, Fakhfouri G, Balzaretti G, Marcello E, Gardoni F, Canonico PL, et al. A $\beta$ leads to $\mathrm{Ca}^{2+}$ signaling alterations and transcriptional changes in glial cells. Neurobiology of Aging 34: 511-22.(2013).

[9] Lim D, Iyer A, Ronco V, Grolla AA, Canonico PL, Aronica E, et al. Amyloid beta deregulates astroglial mGluR5-mediated calcium signaling via calcineurin and Nf-kB. Glia 61: 1134-45.(2013). [10] Ronco V, Grolla AA, Glasnov TN, Canonico PL, Verkhratsky A, Genazzani AA, et al. Differential deregulation of astrocytic calcium signalling by amyloid- $\beta$, TNF $\alpha$, IL-1 $\beta$ and LPS. Cell Calcium 55: 219-29.(2014).

[11] Grolla AA, Sim JA, Lim D, Rodriguez JJ, Genazzani AA, Verkhratsky A. Amyloid- $\beta$ and Alzheimer's disease type pathology differentially affects the calcium signalling toolkit in astrocytes from different brain regions. Cell Death \& Disease 4: e623.(2013).

[12] Oddo S, Caccamo A, Shepherd JD, Murphy MP, Golde TE, Kayed R, et al. Triple-transgenic model of Alzheimer's disease with plaques and tangles: intracellular Abeta and synaptic dysfunction. Neuron 39: 409-21.(2003).

[13] Smyth GK. Linear models and empirical bayes methods for assessing differential expression in microarray experiments. Statistical Applications in Genetics and Molecular Biology 3: Article3.(2004). 
[14] Benjamini Y, Hochberg Y. Controlling the False Discovery Rate: A Practical and Powerful Approach to Multiple Testing. Journal of the Royal Statistical Society Series B (Methodological) 57: 289-300.(1995).

[15] Bourgon R, Gentleman R, Huber W. Independent filtering increases detection power for highthroughput experiments. Proceedings of the National Academy of Sciences of the United States of America 107: 9546-51.(2010).

[16] Huang DW, Sherman BT, Lempicki RA. Systematic and integrative analysis of large gene lists using DAVID bioinformatics resources. Nature Protocols 4: 44-57.(2009).

[17] Cahoy JD, Emery B, Kaushal A, Foo LC, Zamanian JL, Christopherson KS, et al. A transcriptome database for astrocytes, neurons, and oligodendrocytes: a new resource for understanding brain development and function. The Journal of Neuroscience 28: 264-78.(2008).

[18] Walsh CJ, Hu P, Batt J, Santos CCD. Microarray Meta-Analysis and Cross-Platform Normalization: Integrative Genomics for Robust Biomarker Discovery. Microarrays (Basel, Switzerland) 4: 389-406.(2015).

[19] Shabalin AA, Tjelmeland H, Fan C, Perou CM, Nobel AB. Merging two gene-expression studies via cross-platform normalization. Bioinformatics 24: 1154-60.(2008).

[20] Breitling R, Armengaud P, Amtmann A, Herzyk P. Rank products: a simple, yet powerful, new method to detect differentially regulated genes in replicated microarray experiments. FEBS letters 573: 83-92.(2004).

[21] Orre M, Kamphuis W, Osborn LM, Melief J, Kooijman L, Huitinga I, et al. Acute isolation and transcriptome characterization of cortical astrocytes and microglia from young and aged mice. Neurobiology of Aging 35: 1-14.(2014).

[22] Berchtold NC, Sabbagh MN, Beach TG, Kim RC, Cribbs DH, Cotman CW. Brain gene expression patterns differentiate mild cognitive impairment from normal aged and Alzheimer's disease. Neurobiology of Aging 35: 1961-72.(2014).

[23] Saeed AI, Sharov V, White J, Li J, Liang W, Bhagabati N, et al. TM4: a free, open-source system for microarray data management and analysis. BioTechniques 34: 374-78.(2003).

[24] Chang L-C, Lin H-M, Sibille E, Tseng GC. Meta-analysis methods for combining multiple expression profiles: comparisons, statistical characterization and an application guideline. BMC bioinformatics 14: 368.(2013).

[25] Hertz L, Chen Y, Song D. Astrocyte Cultures Mimicking Brain Astrocytes in Gene Expression, Signaling, Metabolism and K+ Uptake and Showing Astrocytic Gene Expression Overlooked by Immunohistochemistry and In Situ Hybridization. Neurochemical Research 42: 25471.(2017). 
[26] Guo Q, Fu W, Sopher BL, Miller MW, Ware CB, Martin GM, et al. Increased vulnerability of hippocampal neurons to excitotoxic necrosis in presenilin-1 mutant knock-in mice. Nature Medicine 5: 101-06.(1999).

[27] van Gijsel-Bonnello M, Baranger K, Benech P, Rivera S, Khrestchatisky M, de Reggi M, et al. Metabolic changes and inflammation in cultured astrocytes from the 5xFAD mouse model of Alzheimer's disease: Alleviation by pantethine. PloS One 12: e0175369.(2017).

[28] Wang H-M, Zhang T, Huang J-K, Sun X-J. 3-N-butylphthalide (NBP) attenuates the amyloid$\beta$-induced inflammatory responses in cultured astrocytes via the nuclear factor- $\kappa \mathrm{B}$ signaling pathway. Cellular Physiology and Biochemistry 32: 235-42.(2013).

[29] Tapella L, Cerruti M, Biocotino I, Stevano A, Rocchio F, Canonico PL, et al. TGF- $\beta 2$ and TGF- $\beta 3$ from cultured $\beta$-amyloid-treated or 3xTg-AD-derived astrocytes may mediate astrocyteneuron communication. The European Journal of Neuroscience 47: 211-21.(2018).

[30] Baird JW, Nibbs RJ, Komai-Koma M, Connolly JA, Ottersbach K, Clark-Lewis I, et al. ESkine, a novel beta-chemokine, is differentially spliced to produce secretable and nuclear targeted isoforms. The Journal of Biological Chemistry 274: 33496-503.(1999).

[31] Nibbs RJB, Graham GJ. CCL27/PESKY: a novel paradigm for chemokine function. Expert Opinion on Biological Therapy 3: 15-22.(2003).

[32] Schubert P, Morino T, Miyazaki H, Ogata T, Nakamura Y, Marchini C, et al. Cascading glia reactions: a common pathomechanism and its differentiated control by cyclic nucleotide signaling. Annals of the New York Academy of Sciences 903: 24-33.(2000).

[33] Balducci C, Frasca A, Zotti M, La Vitola P, Mhillaj E, Grigoli E, et al. Toll-like receptor 4dependent glial cell activation mediates the impairment in memory establishment induced by $\beta$ amyloid oligomers in an acute mouse model of Alzheimer's disease. Brain, Behavior, and Immunity 60: 188-97.(2017).

[34] Simpson JE, Ince PG, Shaw PJ, Heath PR, Raman R, Garwood CJ, et al. Microarray analysis of the astrocyte transcriptome in the aging brain: relationship to Alzheimer's pathology and APOE genotype. Neurobiology of Aging 32: 1795-807.(2011).

[35] Lim D, Ronco V, Grolla AA, Verkhratsky A, Genazzani AA. Glial calcium signalling in Alzheimer's disease. Reviews of Physiology, Biochemistry and Pharmacology 167: 45-65.(2014). [36] Perluigi M, Di Domenico F, Butterfield DA. mTOR signaling in aging and neurodegeneration: At the crossroad between metabolism dysfunction and impairment of autophagy. Neurobiology of Disease 84: 39-49.(2015).

[37] Grimm A, Friedland K, Eckert A. Mitochondrial dysfunction: the missing link between aging and sporadic Alzheimer's disease. Biogerontology 17: 281-96.(2016). 
[38] Maiese K. Taking aim at Alzheimer's disease through the mammalian target of rapamycin. Annals of Medicine 46: 587-96.(2014).

[39] Kiriyama Y, Nochi H. The Function of Autophagy in Neurodegenerative Diseases. International Journal of Molecular Sciences 16: 26797-812.(2015).

[40] Chen L, Dumelie JG, Li X, Cheng MH, Yang Z, Laver JD, et al. Global regulation of mRNA translation and stability in the early Drosophila embryo by the Smaug RNA-binding protein. Genome Biology 15: R4.(2014).

[41] Chen Z, Holland W, Shelton JM, Ali A, Zhan X, Won S, et al. Mutation of mouse Samd4 causes leanness, myopathy, uncoupled mitochondrial respiration, and dysregulated mTORC1 signaling. Proceedings of the National Academy of Sciences of the United States of America 111: 7367-72.(2014).

[42] Stenovec M, Trkov S, Lasič E, Terzieva S, Kreft M, Rodríguez Arellano JJ, et al. Expression of familial Alzheimer disease presenilin 1 gene attenuates vesicle traffic and reduces peptide secretion in cultured astrocytes devoid of pathologic tissue environment. Glia 64: 317-29.(2016). [43] Verkhratsky A, Sofroniew MV, Messing A, deLanerolle NC, Rempe D, Rodríguez JJ, et al. Neurological diseases as primary gliopathies: a reassessment of neurocentrism. ASN neuro 42012). [44] Soreq L, Consortium UBE, Consortium NABE, Rose J, Soreq E, Hardy J, et al. Major Shifts in Glial Regional Identity Are a Transcriptional Hallmark of Human Brain Aging. Cell Reports 18: 557-70.(2017).

[45] Barbash S, Garfinkel BP, Maoz R, Simchovitz A, Nadorp B, Guffanti A, et al. Alzheimer's brains show inter-related changes in RNA and lipid metabolism. Neurobiology of Disease 106: 113.(2017). 


\section{Appendix}

\section{Common significant gene analysis}

Given a statistical criterion for significance assessment and a dataset $\mathrm{X}$ featuring $\mathrm{x}_{\mathrm{s}}$ significantly regulated genes, one might be interested in comparing it with another dataset $\mathrm{Y}$ (containing at least each of the $\mathrm{x}_{\mathrm{s}}$ genes) in order to find out how many of those $\mathrm{x}_{\mathrm{s}}$ genes are also significantly regulated in $\mathrm{Y}$ and how much this overlap is likely to be random, rather than representative of a meaningful common transcriptional pattern.

We say:

- $\mathrm{x}_{\mathrm{up}}$ and $\mathrm{x}_{\mathrm{down}}$ the number of genes significantly up- and down-regulated in the dataset $\mathrm{X}$, being $\mathrm{x}_{\mathrm{s}}=\mathrm{x}_{\mathrm{up}}+$ $\mathrm{x}_{\mathrm{down}}$ the total number of genes that passed the test for significance assessment within dataset $\mathrm{X}$;

- $\mathrm{y}_{\mathrm{up}}$ and $\mathrm{y}_{\mathrm{down}}$ the number of genes significantly up- and down-regulated in the dataset $\mathrm{Y}$, being $\mathrm{y}_{\mathrm{s}}=\mathrm{y}_{\mathrm{up}}+$ $\mathrm{y}_{\mathrm{down}}$ the total number of genes that passed the test for significance assessment within dataset $\mathrm{Y}$;

- $\mathrm{n}_{\mathrm{y}}$ the total number of genes present in $\mathrm{Y}$ dataset; it will be necessarily $\mathrm{n}_{\mathrm{y}} \geq \mathrm{y}_{\mathrm{s}}$, even if typically is $\mathrm{n}_{\mathrm{y}}>>$ $\mathrm{y}_{\mathrm{s}}$; moreover, since $\mathrm{Y}$ contains each of the $\mathrm{x}_{\mathrm{s}}$ genes by hypothesis, it will also be $\mathrm{n}_{\mathrm{y}} \geq \mathrm{x}_{\mathrm{s}}$.

- $\mathbf{E}[z]$ the expectation value of the discrete random variable $\mathrm{z}$.

In this problem we can have basically 6 categories of genes:

\begin{tabular}{|l|l|l|}
\hline$U U$ & $U 0$ & $U D$ \\
\hline$D D$ & $D 0$ & $D U$ \\
\hline
\end{tabular}

- $U U=$ experimental number of genes that are significantly up-regulated in $\mathrm{X}$ and significantly upregulated in Y (upward co-regulation);

- $D D=$ experimental number of genes that are significantly down-regulated in $\mathrm{X}$ and significantly downregulated in Y (downward co-regulation);

- $U 0=$ experimental number of genes that are significantly up-regulated in $\mathrm{X}$ but are not significant in $\mathrm{Y}$ ("up-to-zero");

- $D 0=$ experimental number of genes that are significantly down-regulated in $\mathrm{X}$ but are not significant in Y (“down-to-zero");

- $U D=$ experimental number of genes that are significantly up-regulated in $\mathrm{X}$ and significantly downregulated in Y ("up-to-down" anti-regulation);

- $D U=$ experimental number of genes that are significantly down-regulated in $\mathrm{X}$ and significantly upregulated in Y (“down-to-up" anti-regulation).

Notice that the problem we are addressing is not fully symmetrical in that we are not interested in those genes that are not significant in $\mathrm{X}$ (typically $\mathrm{X}$ is the dataset to be studied, while $\mathrm{Y}$ is an auxiliary dataset used as a reference, comparison or control). Also notice that $\mathrm{x}_{\mathrm{s}}$ does not necessarily have to be the full set of significantly regulated genes in $\mathrm{X}$, but just the largest subset suitable for the comparison (i.e.: \{significant genes in $\mathrm{X}\} \cap \mathrm{Y})$.

If $\mathrm{X}$ and $\mathrm{Y}$ are independent (e.g. they describe the transcriptome of two completely unrelated pathologies) there should not be any correlation between them, that is to say that significant genes are randomly distributed in the two datasets (at least mutually). Using the previous notations, assuming that $\mathrm{X}$ and $\mathrm{Y}$ are independent and that both $\mathrm{X}$ and $\mathrm{Y}$ provide a uniform (even though incomplete) coverage of the transcriptome, starting from the experimental occurrences of the genes called as significant, we can easily 
predict how many genes we should expect to find by chance in each one of the 6 categories (lower case is used to indicate a discrete random variable):

\begin{tabular}{|c|c|c|}
\hline $\mathbf{E}[u u]=x_{u p} \frac{y_{u p}}{n_{y}}$ & $\mathbf{E}[u 0]=x_{u p}\left(1-\frac{y_{s}}{n_{y}}\right)$ & $\mathbf{E}[u d]=x_{u p} \frac{y_{d o w n}}{n_{y}}$ \\
\hline $\mathbf{E}[d d]=x_{\text {down }} \frac{y_{\text {down }}}{n_{y}}$ & $\mathbf{E}[d 0]=x_{\text {down }}\left(1-\frac{y_{s}}{n_{y}}\right)$ & $\mathbf{E}[d u]=x_{\text {down }} \frac{y_{u p}}{n_{y}}$ \\
\hline
\end{tabular}

By this way we can define a randomized null model based on the empirical proportions of significant genes in $\mathrm{X}$ and $\mathrm{Y}$, the only assumption being the mutually independence of $\mathrm{X}$ and $\mathrm{Y}$. In order to compare empirical data $\{U U, U 0, U D, D D, D 0, D U\}$ to the expected values predicted by the null model, we also need a statistical model describing how the 6 random variables $\{u u, u 0, u d, d d, d 0, d u\}$ distribute around their expected values. Binomial distribution can be used to this purpose: $\operatorname{Pr}(k)=\left(\begin{array}{l}n \\ k\end{array}\right) p^{k}(1-p)^{n-k}$, where $\left(\begin{array}{l}n \\ k\end{array}\right)=\frac{n !}{k !(n-k) !}$ is the binomial coefficient, represents the probability mass function describing the probability of getting exactly $k$ successes in $n$ trials, $p$ being the probability of a single success in a single trial. In our case a success is intended to be one of the 6 possible gene combinations, $n$ is $\mathrm{x}_{\text {up }}$ or $\mathrm{x}_{\text {down }}$, while $p$ is equal to $\mathrm{y}_{\mathrm{up}} / \mathrm{n}_{\mathrm{y}}$ rather than $\mathrm{y}_{\text {down }} / \mathrm{n}_{\mathrm{y}}$, depending on the particular combination of interest. So we have:

$$
\begin{aligned}
& \operatorname{Pr}(u u=k)=\left(\begin{array}{c}
x_{u p} \\
k
\end{array}\right)\left(\frac{y_{u p}}{n_{y}}\right)^{k}\left(1-\frac{y_{u p}}{n_{y}}\right)^{x_{u p}-k}, \\
& \operatorname{Pr}(u 0=k)=\left(\begin{array}{c}
x_{u p} \\
k
\end{array}\right)\left(1-\frac{y_{s}}{n_{y}}\right)^{k}\left(\frac{y_{s}}{n_{y}}\right)^{x_{u p}-k}, \\
& \operatorname{Pr}(u d=k)=\left(\begin{array}{c}
x_{u p} \\
k
\end{array}\right)\left(\frac{y_{\text {down }}}{n_{y}}\right)^{k}\left(1-\frac{y_{\text {down }}}{n_{y}}\right)^{x_{u p}-k}, \\
& \operatorname{Pr}(d d=k)=\left(\begin{array}{c}
x_{\text {down }} \\
k
\end{array}\right)\left(\frac{y_{\text {down }}}{n_{y}}\right)^{k}\left(1-\frac{y_{\text {down }}}{n_{y}}\right)^{x_{\text {down }}-k}, \\
& \operatorname{Pr}(d 0=k)=\left(\begin{array}{c}
x_{\text {down }} \\
k
\end{array}\right)\left(1-\frac{y_{s}}{n_{y}}\right)^{k}\left(\frac{y_{s}}{n_{y}}\right)^{x_{\text {down }}-k}, \\
& \operatorname{Pr}(d u=k)=\left(\begin{array}{c}
x_{\text {down }} \\
k
\end{array}\right)\left(\frac{y_{u p}}{n_{y}}\right)^{k}\left(1-\frac{y_{u p}}{n_{y}}\right)^{x_{\text {down }}-k} .
\end{aligned}
$$

Notice that, being $\mathbf{E}[k]=n p$ the mean of a binomially distributed random variable, these probability mass functions are consistent with the expectation values already showed in table.

Actually, in order to estimate the probability of see a particular experimental configuration $\{U U, U 0, U D$, $D D, D O, D U\}$ under the null hypothesis that $\mathrm{X}$ and $\mathrm{Y}$ are independent, we are more interested in the cumulative distribution functions, rather than probability mass functions. In particular, for the first combination $U U$, we have:

$\operatorname{Pr}(u u \geq U U)=\sum_{k=U U}^{x_{u p}}\left(\begin{array}{c}x_{u p} \\ k\end{array}\right)\left(\frac{y_{u p}}{n_{y}}\right)^{k}\left(1-\frac{y_{u p}}{n_{y}}\right)^{x_{u p}-k}$.

Notably, $\operatorname{Pr}(u u \geq U U)$ can be regarded as a $p$-value concerning the enrichment of the entire category containing those genes that are significantly up-regulated in both $\mathrm{X}$ and $\mathrm{Y}$ (upward co-regulation). We call it "categorical $p$-value" (to distinguish it from ordinary $p$-values referred to individual genes) since it answers 
the question: "How likely is it to have $U U$ (or more) genes upwardly co-regulated, under the hypothesis that significant genes are randomly distributed in the two datasets?"

While the same can be said for the other "lateral" categories of genes ( $U D, D D$ and $D U)$, the interest in the two "central" categories (those featuring genes that are significantly regulated only in X) concerns their possible "impoverishment" rather than enrichment. In this case, the statistical significance (categorical $p$ value) can be calculated as follow:

$\operatorname{Pr}(u 0 \leq U 0)=\sum_{k=0}^{U 0}\left(\begin{array}{c}x_{u p} \\ k\end{array}\right)\left(1-\frac{y_{s}}{n_{y}}\right)^{k}\left(\frac{y_{s}}{n_{y}}\right)^{x_{u p}-k}$, and analogously for $D O$

Notice that, in this kind of problem, the 6 categories of interest are not mutually independent. In particular, because of the 2 constraints $x_{u p}=u u+u d+u 0$ and $x_{\text {down }}=d d+d u+d 0$ only 4 categories out of 6 are actually independent, and the same applies to the enrichment hypothesis tests. Thus, since each comparative analysis of two datasets implies 4 independent hypothesis tests, we can adjust categorical $p$-values for multiple comparisons according to the usual correction techniques: Adj.p.val $=4 \cdot p$-value (Bonferroni), Adj.p.val $=1-(1-p \text {-value })^{4}$ (Dunn-Šidák), etc.

Incidentally, because of the same two constraints cited above, the expectation values of the central categories could be alternatively computed as:

$\mathrm{E}[u 0]=x_{u p}-\mathrm{E}[u u]-\mathrm{E}[u d]$,

$\mathbf{E}[d 0]=x_{d o w n}-\mathbf{E}[d d]-\mathbf{E}[d u]$

Even if the two categories $U O$ and $D O$ can be further explored and biologically validated regardless of their categorical $p$-values (simply because they are in any case the containers of the specific gene signature, namely those genes specific for the condition studied by X respect to Y), a significant impoverishment of one of them is suggestive of some kind of correlation between $\mathrm{X}$ and $\mathrm{Y}$ (and hence between the pathologies or treatments they represent). On the contrary, the $p$-values assigned to the lateral categories allow to precisely locate any possible (and statistically significant) overlap of the two dataset (common gene signature), and further investigations should not be justified in the absence of a sufficiently low categorical $p$-value. 


\section{Legends}

Figure 1. Microarray setup. A, Immunofluorescence photographs showing astrocytes marked with GFAP (green) and microglial cells marked with Iba1 (red) antibody. Note the absence of microglia after MACS using anti-CD11b-conjugated beads. Bar, $40 \mu \mathrm{m}$. B, a scheme of the microarray setup.

Figure 2. Real-time PCR validation of microarray results. A, real-time PCR validation of genes emerged in microarray was performed on four independent astrocyte cultures. X-axis shows microarray $\log _{2}$ fold change $(\log F C)$ of $3 x T g-A D$ vs non-Tg astrocytes, while $y$-axis shows $\log F C$ of real-time PCR, $n=23$ genes. B, scatterplots of 14 genes exemplifying the results of real-time PCR validation. Date are expressed as mean $\pm \mathrm{SD} \Delta \mathrm{C}_{(\mathrm{t})}$ of 4 samples each run in triplicate.

Figure 3. Comparison with acutely isolated CNS cellular models. (A) Dendrogram showing the positioning of non-Tg astrocyte cultures compared to the other cellular models present the transcriptome database provided by Cahoy et al. (Astros = acutely isolated astrocytes; Neurons = acutely isolated neurons). Dendrograms were obtained through unsupervised hierarchical clustering (Euclidean metric, average linkage). Cahoy's cellular model are labeled in blue. (B) Heatmaps of the 130 most upregulated genes in non-Tg compared to acutely isolated astrocytes (Astros). See Supplementary Table 3 for "Genes enriched in non-Tg compared to acutely isolated astrocytes". (C) Heatmaps of the 230 most downregulated genes in non-Tg compared to acutely isolated astrocytes (Astros). See Supplementary Table 4 for "Genes enriched in acutely isolated astrocytes compared to non-Tg". Notice the high degree of similarity between our cultured model and Cahoy's cultured astroglia. Both heatmaps (and the corresponding gene lists) were obtained through a RP procedure (FDR $\leq 0.005$, two class, unpaired). Expression data were lastly gene-wise normalized (log-mean subtraction) to represent the expression values of each gene in terms of $\log _{2}$ foldenrichment over their mean value across all samples.

Figure 4. Comparison with isolated astrocytes from symptomatic APPswe/PS1dE9 AD mice. The results of the statistical comparison between present microarray dataset and the dataset provided by Orre et al. (2014) are reported in table, according to the 6-category template, the conventional notation and the null model introduced in Materials and Methods section and detailed in Appendix (Common significant gene analysis). In particular, in each box of the table are, from top to bottom, the actual (experimental) number of genes belonging to that particular category, the expectation value according to the null model and the related categorical $p$-value. Above and below the table are the $\log _{2} \mathrm{FC}$ color-coded heatmaps of the top- 20 regulated genes for each category $(\mathrm{X}=3 \mathrm{xTg}-\mathrm{AD}$ vs non-Tg dataset; $\mathrm{Y}=\mathrm{APP}$ swe/PS1dE9 AD vs WT dataset $)$. A the bottom of the Figure, as an example for the first category (Up-Up = upward co-regulated genes) provided the complete procedure for the calculation of the expectation value and the categorical $p$-value from the null model is shown. Following the arrow: (1) the binomial probability mass function representing the null model for the set of upward co-regulated genes (as given in Appendix: Common significant gene analysis); (2) the numerical values for the constants $\mathrm{x}_{\mathrm{up}}, \mathrm{y}_{\mathrm{up}}$ and $\mathrm{n}_{\mathrm{y}}$ (see Materials and Methods, section Comparison with 
1

2

3

4

5

6

7

8

9

10

11

12

13

14

15

16

17

18

19

20

21

22

23

24

25

26

27

28

29

30

31

32

33

34

35

36

37

38

39

40

41

42

43

44

45

46

47

48

49

50

51

52

53

54

55

56

57

58

59

60

APPswe/PS1dE9 dataset); (3) mean (or expected value), standards deviation and histogram for the null model-binomial distribution; (4) the cumulative distribution function (CDF) in its symbolic form (upper) and evaluated (lower) for the particular experimental value $U U=23$. CDF value corresponds to the red section of the histogram and it is equal to the categorical $p$-value shown in the table. 
Table 1. Top 40 of up- and down-regulated genes in 3xTg-AD astrocytes vs non-Tg.

\begin{tabular}{|c|c|c|c|c|c|}
\hline & Probe Name & Gene Symbol & FC & Ave Expr & adj.P.Val \\
\hline & \multicolumn{5}{|c|}{ Up-regulated genes } \\
\hline 1 & A_52_P267391 & Trim12a & 40.13 & 7.97 & $1.515 \mathrm{E}-21$ \\
\hline 2 & A_55_P1974432 & Gm5067 & 26.12 & 7.94 & 4.182E-23 \\
\hline 3 & A_55_P2070576 & $\mathrm{Ccl} 27 \mathrm{a}$ & 8.94 & 8.53 & $2.516 \mathrm{E}-15$ \\
\hline 4 & A_55_P2205650 & 2610507I01Rik & 6.80 & 8.20 & $1.896 \mathrm{E}-15$ \\
\hline 5 & A_55_P2064659 & Trim12a & 6.17 & 7.01 & 6.074E-14 \\
\hline 6 & A_55_P2256646 & C130078N14 & 5.96 & 6.98 & $4.552 \mathrm{E}-12$ \\
\hline 7 & A_55_P2068731 & Gm20878 & 5.80 & 9.50 & $5.665 \mathrm{E}-10$ \\
\hline 8 & A_52_P360330 & Map1b & 5.77 & 9.57 & $2.685 \mathrm{E}-12$ \\
\hline 9 & A_55_P2068733 & Gm20878 & 5.49 & 8.49 & $5.030 \mathrm{E}-15$ \\
\hline 10 & A_55_P2092750 & Car9 & 4.68 & 11.43 & $1.138 \mathrm{E}-02$ \\
\hline 11 & A_55_P1981756 & Vmn2r123 & 4.67 & 6.86 & $6.992 \mathrm{E}-09$ \\
\hline 12 & A_51_P349888 & Ang2 & 4.66 & 7.46 & $1.004 \mathrm{E}-08$ \\
\hline 13 & A_52_P88793 & Zfp933 & 4.56 & 8.10 & $1.763 \mathrm{E}-14$ \\
\hline 14 & A_52_P238846 & Bpnt1 & 4.53 & 8.50 & 7.344E-09 \\
\hline 15 & A_51_P288549 & Jmjd7 & 4.46 & 9.04 & $9.524 \mathrm{E}-12$ \\
\hline 16 & A_66_P130730 & Zfp963 & 4.42 & 7.71 & $2.516 \mathrm{E}-15$ \\
\hline 17 & A_55_P2112986 & K1k1b22 & 4.41 & 6.95 & $1.265 \mathrm{E}-13$ \\
\hline 18 & A_55_P2134591 & BC049715 & 4.36 & 7.12 & $3.811 \mathrm{E}-16$ \\
\hline 19 & A_55_P2097151 & Ptchd2 & 4.00 & 7.33 & $1.714 \mathrm{E}-13$ \\
\hline 20 & A_51_P277006 & Chst8 & 4.00 & 9.51 & 5.274E-04 \\
\hline 21 & A_55_P2073965 & BC049715 & 3.98 & 6.93 & $1.327 \mathrm{E}-15$ \\
\hline 22 & A_51_P217498 & Slc2a4 & 3.91 & 8.57 & 8.451E-09 \\
\hline 23 & A_55_P2045886 & Stk3 & 3.78 & 9.20 & 6.396E-07 \\
\hline 24 & A_55_P2180869 & Ocel1 & 3.77 & 11.21 & $6.741 \mathrm{E}-11$ \\
\hline 25 & A_55_P2108773 & 4930427A07Rik & 3.74 & 6.87 & $5.035 \mathrm{E}-13$ \\
\hline 26 & A_66_P105689 & Trim34a & 3.56 & 8.37 & $1.752 \mathrm{E}-08$ \\
\hline 27 & A_66_P134474 & Ang3 & 3.48 & 7.97 & $1.877 \mathrm{E}-06$ \\
\hline 28 & A_55_P1964628 & LOC102633627 & 3.40 & 13.64 & $1.627 \mathrm{E}-08$ \\
\hline 29 & A_51_P293069 & Mfsd7b & 3.36 & 9.74 & $9.791 \mathrm{E}-06$ \\
\hline 30 & A_55_P2085142 & Spp1 & 3.31 & 9.07 & $1.515 \mathrm{E}-04$ \\
\hline 31 & A_55_P2154387 & Bmp4 & 3.31 & 12.83 & $2.007 \mathrm{E}-03$ \\
\hline 32 & A_51_P413147 & Klk1b3 & 3.25 & 6.73 & $1.200 \mathrm{E}-10$ \\
\hline 33 & A_51_P112627 & St6galnac2 & 3.23 & 10.19 & 9.909E-05 \\
\hline 34 & A_51_P512820 & Dera & 3.21 & 8.49 & $1.344 \mathrm{E}-05$ \\
\hline 35 & A_55_P2095603 & Ccdc65 & 3.21 & 10.33 & $1.100 \mathrm{E}-06$ \\
\hline 36 & A_51_P477121 & Pmaip1 & 3.16 & 9.69 & $1.936 \mathrm{E}-03$ \\
\hline 37 & A_55_P2130129 & Kcnab1 & 3.14 & 9.03 & $2.465 \mathrm{E}-04$ \\
\hline 38 & A_55_P2373852 & 2310058N22Rik & 3.04 & 8.18 & $1.926 \mathrm{E}-09$ \\
\hline 39 & A_55_P2144597 & 9030025P20Rik & 2.98 & 10.33 & 4.017E-06 \\
\hline 40 & A_51_P180724 & Mlh1 & 2.94 & 10.33 & 1.772E-14 \\
\hline
\end{tabular}


Table 1 (Continue).

\begin{tabular}{|c|c|c|c|c|c|}
\hline & ProbeName & GeneSymbol & FC & AveExpr & adj.P.Val \\
\hline \multicolumn{6}{|c|}{ Down-regulated genes } \\
\hline 1 & A_55_P1966838 & Xaf1 & -71.35 & 9.99 & $1.65 \mathrm{E}-09$ \\
\hline 2 & A_52_P516409 & Col4a6 & -20.09 & 11.49 & $1.69 \mathrm{E}-16$ \\
\hline 3 & A_55_P1966774 & Serpina3i & -11.45 & 8.24 & $1.11 \mathrm{E}-03$ \\
\hline 4 & A_66_P130813 & Samd4 & -10.42 & 9.71 & $3.48 \mathrm{E}-12$ \\
\hline 5 & A_52_P303176 & 1810037I17Rik & -9.31 & 12.76 & $9.75 \mathrm{E}-16$ \\
\hline 6 & A_55_P2132207 & 1810037I17Rik & -8.88 & 11.50 & $8.74 \mathrm{E}-16$ \\
\hline 7 & A_55_P2137527 & Fam183b & -8.85 & 10.89 & 4.38E-06 \\
\hline 8 & A_52_P111031 & Pcdh17 & -8.55 & 9.29 & $4.62 \mathrm{E}-09$ \\
\hline 9 & A_55_P2026420 & Pou6f1 & -8.49 & 8.57 & $1.53 \mathrm{E}-11$ \\
\hline 10 & A_52_P206492 & Pop4 & -7.95 & 11.38 & $1.20 \mathrm{E}-16$ \\
\hline 11 & A_55_P2105944 & Olfr224 & -7.23 & 8.06 & $2.77 \mathrm{E}-14$ \\
\hline 12 & A_55_P1952482 & $\mathrm{Pbp} 2$ & -6.54 & 7.72 & $5.33 \mathrm{E}-10$ \\
\hline 13 & A_52_P393314 & P2rx7 & -5.92 & 9.92 & $1.55 \mathrm{E}-05$ \\
\hline 14 & A_51_P462428 & Galnt15 & -5.02 & 10.82 & $1.10 \mathrm{E}-05$ \\
\hline 15 & A_55_P2026270 & Cfi & -4.87 & 7.60 & $8.63 \mathrm{E}-04$ \\
\hline 16 & A_51_P159453 & Serpina3n & -4.78 & 11.29 & $2.24 \mathrm{E}-02$ \\
\hline 17 & A_52_P613498 & 4833420G17Rik & -4.39 & 11.73 & $2.19 \mathrm{E}-10$ \\
\hline 18 & A_52_P157450 & Abhd1 & -4.08 & 9.15 & $1.95 \mathrm{E}-13$ \\
\hline 19 & A_51_P297105 & Ucp2 & -4.02 & 9.28 & $3.45 \mathrm{E}-03$ \\
\hline 20 & A_55_P2074656 & Padi2 & -3.95 & 13.12 & $2.85 \mathrm{E}-06$ \\
\hline 21 & A_52_P257812 & Lpl & -3.91 & 8.51 & $3.96 \mathrm{E}-04$ \\
\hline 22 & A_51_P259296 & Lpl & -3.89 & 10.54 & $2.14 \mathrm{E}-03$ \\
\hline 23 & A_52_P597775 & Gprc5a & -3.69 & 10.69 & $4.87 \mathrm{E}-05$ \\
\hline 24 & A_55_P2091359 & Padi2 & -3.54 & 12.93 & $9.79 \mathrm{E}-06$ \\
\hline 25 & A_55_P2045642 & Stmn4 & -3.40 & 9.27 & $1.70 \mathrm{E}-02$ \\
\hline 26 & A_66_P108247 & Ucp3 & -3.32 & 8.74 & $5.58 \mathrm{E}-03$ \\
\hline 27 & A_55_P2176792 & Sh3gl3 & -3.31 & 9.35 & $1.20 \mathrm{E}-10$ \\
\hline 28 & A_66_P105175 & Bche & -3.29 & 10.68 & $1.00 \mathrm{E}-08$ \\
\hline 29 & A_51_P128667 & Lynx1 & -3.29 & 11.50 & $8.91 \mathrm{E}-07$ \\
\hline 30 & A_51_P194249 & Stmn4 & -3.23 & 9.25 & $3.23 \mathrm{E}-02$ \\
\hline 31 & A_55_P2212603 & Apba2 & -3.22 & 9.23 & $6.98 \mathrm{E}-05$ \\
\hline 32 & A_55_P1953972 & Pdhb & -3.21 & 12.85 & $1.48 \mathrm{E}-09$ \\
\hline 33 & A_55_P1978465 & H2-Q5 & -3.17 & 9.54 & $5.66 \mathrm{E}-03$ \\
\hline 34 & A_55_P2315012 & 4930458D05Rik & -3.16 & 7.04 & $1.12 \mathrm{E}-07$ \\
\hline 35 & A_55_P1968276 & Tomm22 & -3.08 & 12.76 & $5.03 \mathrm{E}-15$ \\
\hline 36 & A_55_P2096867 & Gap43 & -3.05 & 10.06 & $2.29 \mathrm{E}-03$ \\
\hline 37 & A_55_P2004179 & Col2a1 & -3.03 & 10.26 & 3.04E-02 \\
\hline 38 & A_55_P2162344 & Lrsam1 & -3.02 & 11.18 & $5.14 \mathrm{E}-04$ \\
\hline 39 & A_51_P458778 & Hpgd & -2.97 & 7.81 & $4.38 \mathrm{E}-03$ \\
\hline 40 & A_55_P2014304 & Kank1 & -2.95 & 11.85 & $8.04 \mathrm{E}-08$ \\
\hline
\end{tabular}


Table 2. Gene ontology analysis.

\begin{tabular}{|c|c|c|c|c|}
\hline GO Category & Term & Count & \begin{tabular}{|l} 
Fold \\
Enrichment
\end{tabular} & FDR, $\%$ \\
\hline \multicolumn{5}{|c|}{ Up-regulated genes } \\
\hline UP_KEYWORDS & Phosphoprotein & 159 & 1.2 & 4.78 \\
\hline GOTERM_CC_DIRECT & GO:0005737 cytoplasm & 149 & 1.2 & 1.50 \\
\hline UP_KEYWORDS+A2:G25 & Alternative splicing & 118 & 1.4 & 0.04 \\
\hline UP_SEQ_FEATURE & splice variant & 110 & 1.3 & 7.37 \\
\hline UP_KEYWORDS & Cytoplasm & 102 & 1.3 & 1.83 \\
\hline UP_KEYWORDS & Acetylation & 75 & 1.4 & 4.11 \\
\hline UP_KEYWORDS & Transferase & 49 & 1.7 & 0.43 \\
\hline UP_KEYWORDS & Nucleotide-binding & 49 & 1.6 & 1.55 \\
\hline GOTERM_CC_DIRECT & GO:0005739 mitochondrion & 46 & 1.5 & 8.31 \\
\hline GOTERM_MF_DIRECT & \begin{tabular}{|l|} 
GO:0016740 transferase \\
activity
\end{tabular} & 44 & 1.6 & 1.99 \\
\hline GOTERM_CC_DIRECT & GO:0005622 intracellular & 44 & 1.5 & 7.75 \\
\hline UP_KEYWORDS & ATP-binding & 38 & 1.6 & 6.26 \\
\hline UP_KEYWORDS & Mitochondrion & 30 & 1.6 & 9.26 \\
\hline GOTERM_CC_DIRECT & $\begin{array}{l}\text { GO:0048471 perinuclear } \\
\text { region of cytoplasm }\end{array}$ & 26 & 2.1 & 1.21 \\
\hline GOTERM_CC_DIRECT & GO:0042995 cell projection & 23 & 1.8 & 9.77 \\
\hline INTERPRO & $\begin{array}{l}\text { IPR015880:Zinc finger, C2H2- } \\
\text { like }\end{array}$ & 22 & 1.8 & 9.89 \\
\hline INTERPRO & $\begin{array}{l}\text { IPR001909:Krueppel- } \\
\text { associated box }\end{array}$ & 15 & 2.3 & 7.90 \\
\hline UP_SEQ_FEATURE & $\begin{array}{l}\text { compositionally biased } \\
\text { region:Poly-Arg } \\
\end{array}$ & 11 & 3.4 & 2.40 \\
\hline GOTERM_CC_DIRECT & GO:0031982 vesicle & 10 & 3.4 & 4.13 \\
\hline \multicolumn{5}{|c|}{ Down-regulated genes } \\
\hline UP_KEYWORDS & Phosphoprotein & 167 & 1.4 & 0.001 \\
\hline GOTERM_CC_DIRECT & GO:0005737 cytoplasm & 139 & 1.2 & 4.123 \\
\hline UP_KEYWORDS & Alternative splicing & 105 & 1.4 & 0.518 \\
\hline UP_KEYWORDS & Cytoplasm & 98 & 1.4 & 0.581 \\
\hline UP_KEYWORDS & Transport & 46 & 1.5 & 7.127 \\
\hline GOTERM_CC_DIRECT & GO:0005730 nucleolus & 29 & 2.0 & 0.893 \\
\hline GOTERM_CC_DIRECT & GO:0042995 cell projection & 24 & 2.0 & 3.785 \\
\hline UP_KEYWORDS & Calcium & 24 & 1.8 & 9.853 \\
\hline UP_KEYWORDS & Cell projection & 22 & 2.0 & 4.322 \\
\hline GOTERM_BP_DIRECT & $\begin{array}{l}\text { GO:0006915 apoptotic } \\
\text { process }\end{array}$ & 20 & 2.0 & 8.671 \\
\hline UP_KEYWORDS & Cell junction & 20 & 1.9 & 9.435 \\
\hline GOTERM_CC_DIRECT & GO:0045202 synapse & 17 & 2.0 & 7.425 \\
\hline UP_KEYWORDS & Polymorphism & 10 & 3.0 & 8.706 \\
\hline GOTERM_CC_DIRECT & GO:0043197 dendritic spine & 9 & 3.5 & 5.361 \\
\hline GOTERM_BP_DIRECT & \begin{tabular}{|l|} 
GO:0097191 extrinsic \\
apoptotic signaling pathway
\end{tabular} & 5 & 7.0 & 8.711 \\
\hline
\end{tabular}




\begin{tabular}{|l|l|c|c|c|} 
UP_SEQ_FEATURE & domain:EH & 4 & 37.9 & 0.161 \\
\hline SMART & SM00027:EH & 4 & 21.5 & 0.838 \\
\hline INTERPRO & $\begin{array}{l}\text { IPR000261:EPS15 homology } \\
\text { (EH) }\end{array}$ & 4 & 22.4 & 0.950 \\
\hline GOTERM_BP_DIRECT & $\begin{array}{l}\text { GO:0001916 positive } \\
\text { regulation of T cell mediated } \\
\text { cytotoxicity }\end{array}$ & 4 & 12.0 & 6.578 \\
\hline
\end{tabular}

11 
Table 3. List of down-regulated genes related to GO term "Calcium".

\begin{tabular}{|c|c|c|c|c|c|}
\hline ProbeName & GeneSymbol & GeneName & FC & AveExpr & adj.P.Val \\
\hline A_52_P111031 & Pcdh17 & protocadherin 17 & -8.55 & 9.29 & 4.6E-09 \\
\hline A_55_P2026270 & Cfi & complement component factor i & -4.87 & 7.60 & $8.6 \mathrm{E}-04$ \\
\hline A_55_P2074656 & Padi2 & peptidyl arginine deiminase, type II & -3.95 & 13.12 & $2.9 \mathrm{E}-06$ \\
\hline A_55_P2091359 & Padi2 & peptidyl arginine deiminase, type II & -3.54 & 12.93 & $9.8 \mathrm{E}-06$ \\
\hline A_55_P2004179 & Col2a1 & collagen, type II, alpha 1 & -3.03 & 10.26 & $3.0 \mathrm{E}-02$ \\
\hline A_51_P360918 & Ehd3 & EH-domain containing 3 & -2.59 & 10.17 & $1.0 \mathrm{E}-05$ \\
\hline A_51_P382970 & Itga9 & integrin alpha 9 & -2.55 & 8.76 & $9.2 \mathrm{E}-09$ \\
\hline A_55_P2013223 & S100z & S100 calcium binding protein, zeta & -2.51 & 6.93 & $4.5 \mathrm{E}-03$ \\
\hline A_52_P590535 & Fbln2 & fibulin 2 & -2.13 & 12.94 & $3.5 \mathrm{E}-02$ \\
\hline A_55_P2080880 & Clcnkb & chloride channel Kb & -2.13 & 7.37 & $5.2 \mathrm{E}-06$ \\
\hline A_51_P502437 & Cacna2d3 & $\begin{array}{l}\text { calcium channel, voltage- } \\
\text { dependent, alpha2/delta subunit } 3\end{array}$ & -1.95 & 8.02 & $2.1 \mathrm{E}-02$ \\
\hline A_51_P135340 & Panx1 & pannexin 1 & -1.87 & 10.51 & $2.4 \mathrm{E}-02$ \\
\hline A_52_P529195 & Pcdhb4 & protocadherin beta 4 & -1.80 & 7.04 & $2.1 \mathrm{E}-05$ \\
\hline A_52_P489295 & Adamts1 & $\begin{array}{l}\text { a disintegrin-like and } \\
\text { metallopeptidase (reprolysin type) } \\
\text { with thrombospondin type } 1 \text { motif, } \\
1\end{array}$ & -1.77 & 12.75 & $2.4 \mathrm{E}-03$ \\
\hline A_55_P2185504 & Masp2 & $\begin{array}{l}\text { mannan-binding lectin serine } \\
\text { peptidase } 2\end{array}$ & -1.69 & 6.99 & $1.2 \mathrm{E}-03$ \\
\hline A_55_P2021266 & Hpse & heparanase & -1.68 & 6.97 & 7.7E-03 \\
\hline A_55_P2007771 & Catsper2 & cation channel, sperm associated 2 & -1.65 & 8.37 & $4.4 \mathrm{E}-02$ \\
\hline A_55_P1985410 & Reps2 & $\begin{array}{l}\text { RALBP1 associated Eps domain } \\
\text { containing protein } 2\end{array}$ & -1.60 & 8.04 & $9.2 \mathrm{E}-05$ \\
\hline A_52_P348031 & Syt9 & synaptotagmin IX & -1.57 & 8.33 & $2.9 \mathrm{E}-03$ \\
\hline A_51_P455807 & Ehd4 & EH-domain containing 4 & -1.52 & 11.96 & $3.3 \mathrm{E}-02$ \\
\hline A_55_P1968858 & Cadps & $\begin{array}{l}\text { Ca2+-dependent secretion } \\
\text { activator }\end{array}$ & -1.51 & 9.34 & $1.4 \mathrm{E}-02$ \\
\hline A_66_P104309 & Myl2 & $\begin{array}{l}\text { myosin, light polypeptide } 2, \\
\text { regulatory, cardiac, slow }\end{array}$ & -1.45 & 7.42 & $1.5 \mathrm{E}-02$ \\
\hline A_55_P2153496 & Ppp2r3d & $\begin{array}{l}\text { protein phosphatase } 2 \text { (formerly } \\
2 A \text { ), regulatory subunit } B^{\prime \prime} \text {, delta }\end{array}$ & -1.38 & 7.11 & $3.8 \mathrm{E}-03$ \\
\hline A_55_P1967500 & Nell1 & NEL-like 1 & -1.38 & 6.77 & $1.4 \mathrm{E}-02$ \\
\hline A_51_P184223 & Pcdhb7 & protocadherin beta 7 & -1.28 & 7.92 & $3.9 \mathrm{E}-02$ \\
\hline
\end{tabular}


A
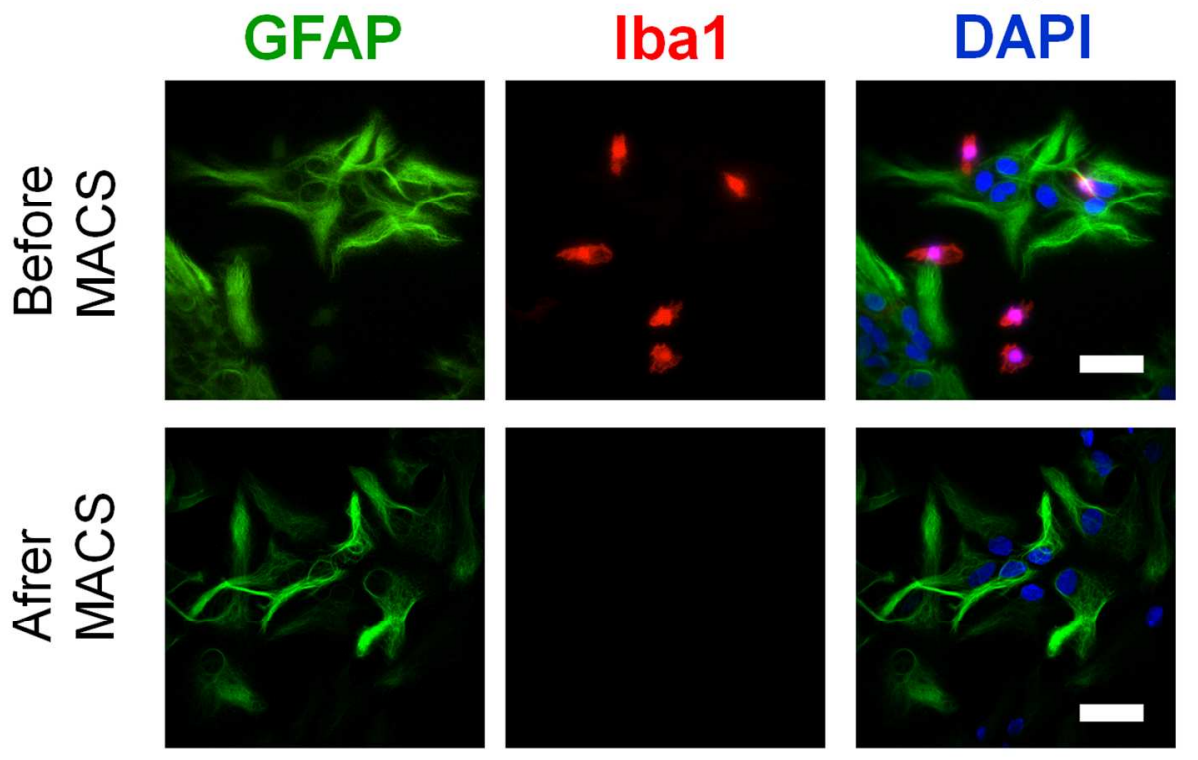

B

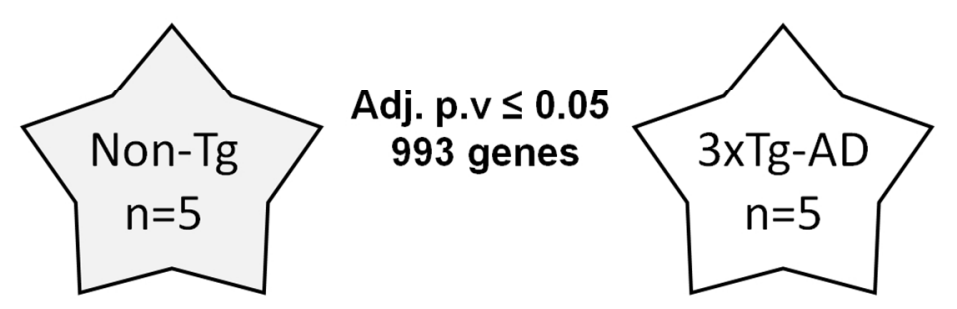

Figure 1. Microarray setup.

$122 \times 143 \mathrm{~mm}(300 \times 300 \mathrm{DPI})$ 
A

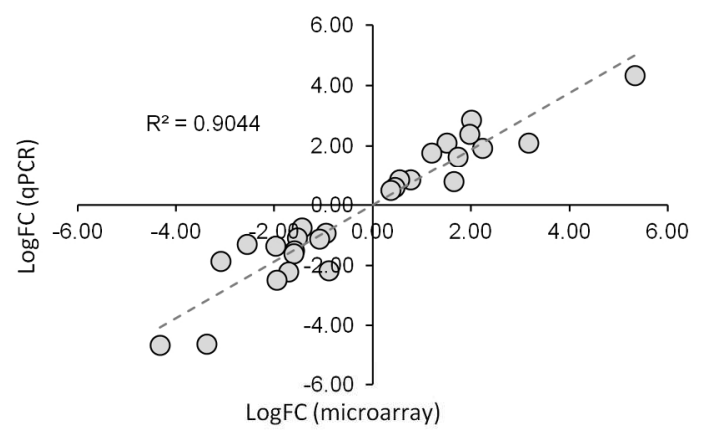

B
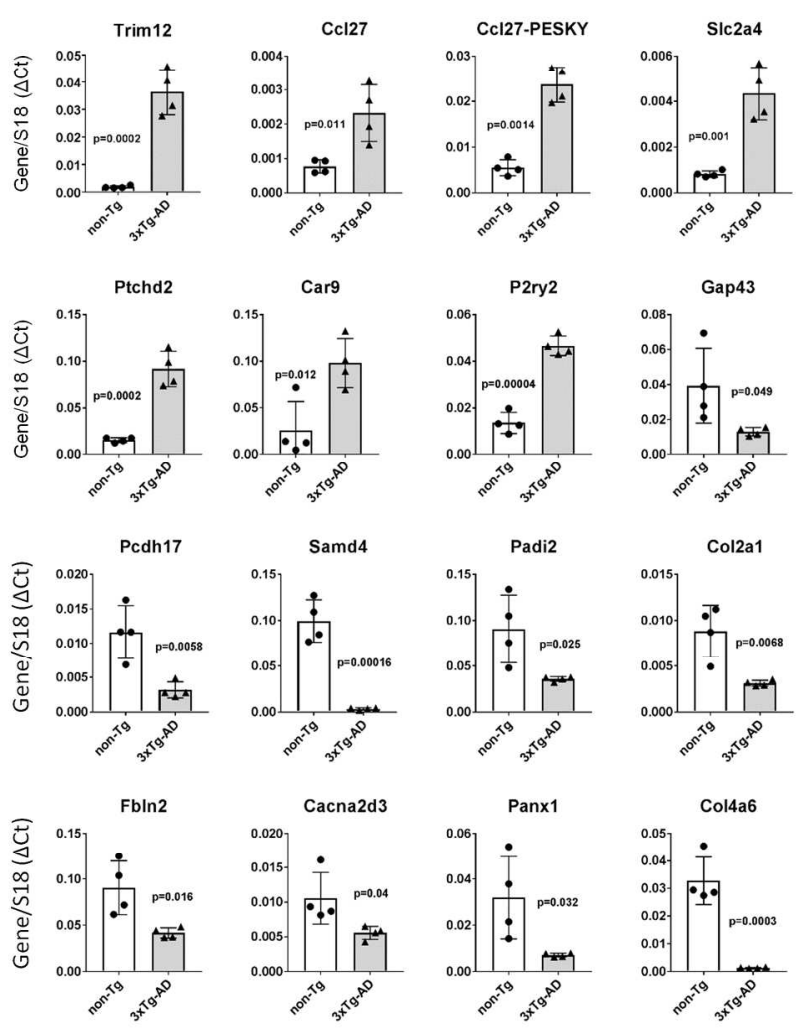

Figure 2. Real-time PCR validation of microarray results. $133 \times 226 \mathrm{~mm}(300 \times 300 \mathrm{DPI})$ 

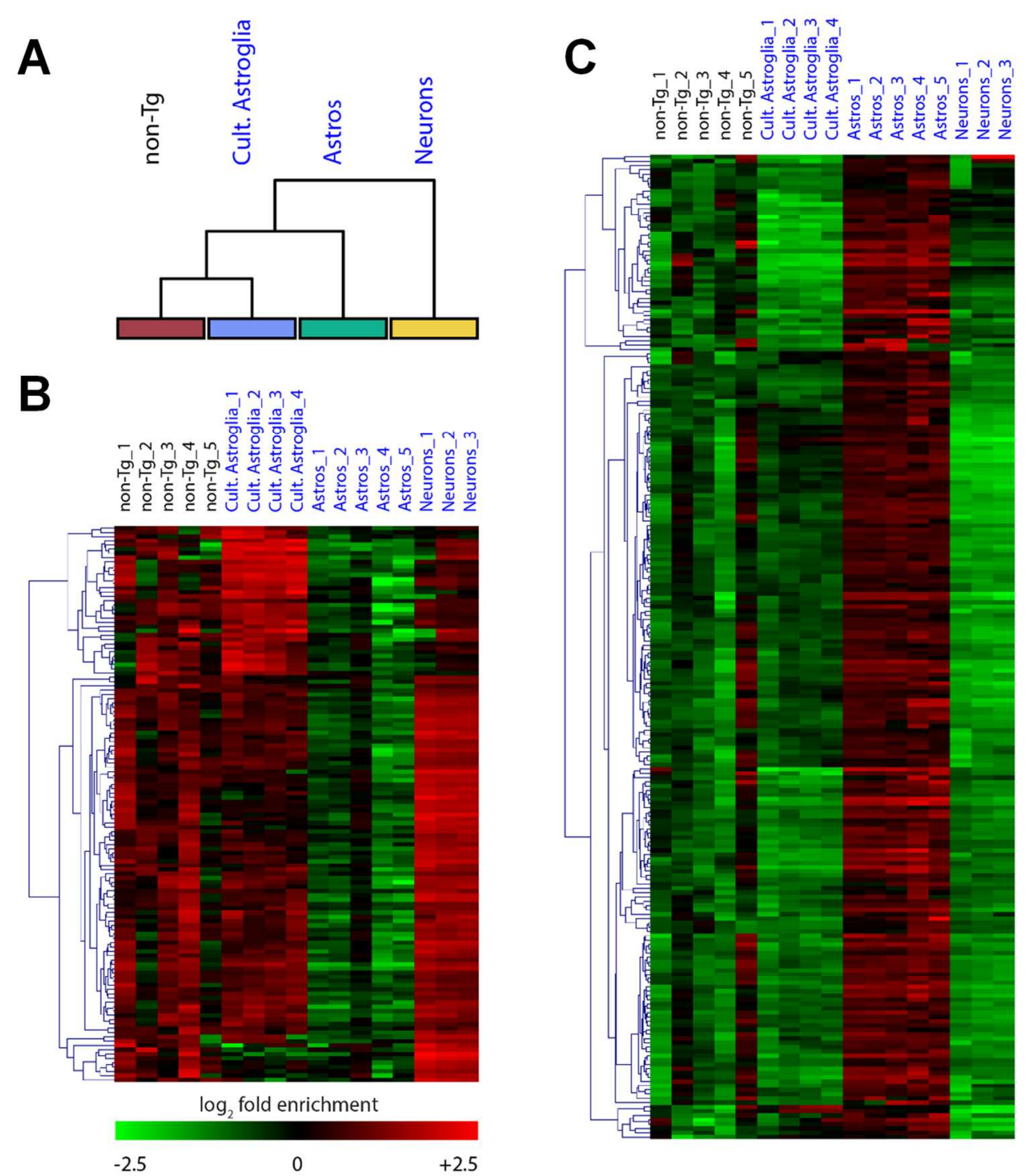

Figure 3. Comparison with acutely isolated CNS cellular models. $150 \times 172 \mathrm{~mm}(300 \times 300 \mathrm{DPI})$ 
Figure 4. Comparison with isolated astrocytes from symptomatic APPswe/PS1dE9 AD mice. $137 \times 217 \mathrm{~mm}(300 \times 300 \mathrm{DPI})$ 


\section{SUPPLEMENTARY MATERIAL}

Transcriptional remodeling in primary hippocampal astrocytes from an Alzheimer's disease mouse model.

Ruffinatti FA, Tapella L, Stevano A, Gregnanin I, Chiorino G, Canonico PL, Distasi C, Genazzani AA, Lim $\mathrm{D}$

Index

\begin{tabular}{|l|l|l|}
\hline Table & Title & File \\
\hline Supplementary Material & $\begin{array}{l}\text { Oligonucleotide primers used for real- } \\
\text { time PCR }\end{array}$ & Supplementary Material \\
\hline Supplementary Table 1 & List of Differentially expressed genes & Supplementary Table 1 \\
\hline Supplementary Table 2 & List of genes validated by real-time PCR & Supplementary Table 2 \\
\hline Supplementary Table 3 & $\begin{array}{l}\text { List of genes up-regulated in non-Tg } \\
\text { compared to acutely isolated astrocytes }\end{array}$ & Supplementary Tables 3-4 \\
\hline Supplementary Table 4 & $\begin{array}{l}\text { List of genes down-regulated in non-Tg } \\
\text { compared to acutely isolated astrocytes }\end{array}$ & Supplementary Tables 3-4 \\
\hline Supplementary Table 5 & $\begin{array}{l}\text { List of genes up-regulated in non-Tg } \\
\text { astrocytes compared to cultured astroglia } \\
\text { from Cahoy's (2014) dataset }\end{array}$ & Supplementary Tables 5-6 \\
\hline Supplementary Table 6 & $\begin{array}{l}\text { List of genes down-regulated in non-Tg } \\
\text { astrocytes compared to cultured astroglia } \\
\text { from Cahoy's (2014) dataset }\end{array}$ & Supplementary Tables 5-6 \\
\hline Supplementary Table 9 & $\begin{array}{l}\text { Comparison with human AD dataset } \\
\text { gists of co- and anti-regulated genes as } \\
\text { compared with Orre et al (2014) }\end{array}$ & Supplementary Table 7 \\
\hline Supplementary Table 8 & $\begin{array}{l}\text { GO analysis of co- and anti-regulated } \\
\text { genes as compared with Orre et al (2014) }\end{array}$ & Supplementary Table 8 \\
\hline Sablentary Table 9 \\
\hline Sury Talement
\end{tabular}




\section{Oligonucleotide primers used for quantitative real-time PCR.}

\begin{tabular}{|c|c|c|c|}
\hline Gene & $\begin{array}{l}\text { Accession } \\
\text { number }\end{array}$ & $\begin{array}{l}\text { Forward } \\
\text { Reverse }\end{array}$ & Sequence 5' to 3' \\
\hline Trim12a & NM_023835.2 & $\begin{array}{l}\text { Forward } \\
\text { Reverse }\end{array}$ & $\begin{array}{l}\text { TCATTGAAGAGGTGGCCCAG } \\
\text { ACCGAACATTCTGCACATCT }\end{array}$ \\
\hline $\mathrm{Ccl} 27 \mathrm{a}$ & NM_011336.1 & $\begin{array}{l}\text { Forward } \\
\text { Reverse }\end{array}$ & $\begin{array}{l}\text { CCCGCTGTTACTGTTGCTTC } \\
\text { CAATCCTCCTCAGCAGCCT }\end{array}$ \\
\hline Ccl27a (Pesky) & NM_001048179.1 & $\begin{array}{l}\text { Forward } \\
\text { Reverse }\end{array}$ & $\begin{array}{l}\text { TCTCCAACAAGCCAGAGACT } \\
\text { GCTTGGGAGTGGCTGTCTAT }\end{array}$ \\
\hline Car9 & NM_139305.2 & $\begin{array}{l}\text { Forward } \\
\text { Reverse }\end{array}$ & $\begin{array}{l}\text { GGAGTCCCTTGGGTTAGAGG } \\
\text { TGGGGCCAGAGTAGGGTG }\end{array}$ \\
\hline Ptchd2 & NM_001083342.1 & $\begin{array}{l}\text { Forward } \\
\text { Reverse }\end{array}$ & $\begin{array}{l}\text { CACAGCCTGCAGAACAATGT } \\
\text { TTGGAGATGTACACGGTGCT }\end{array}$ \\
\hline Slc2a4 & NM_009204.2 & $\begin{array}{l}\text { Forward } \\
\text { Reverse }\end{array}$ & $\begin{array}{l}\text { TAAAACAAGATGCCGTCGGG } \\
\text { CCAAACTGAAGGGAGCCAAG }\end{array}$ \\
\hline Bmp4 & NM_007554.3 & $\begin{array}{l}\text { Forward } \\
\text { Reverse }\end{array}$ & $\begin{array}{l}\text { AGTCTGGGGAGGAGGAGG } \\
\text { GAGCTCTCACTGGTCCCTG }\end{array}$ \\
\hline Kcnab1 & NM_010597.4 & $\begin{array}{l}\text { Forward } \\
\text { Reverse }\end{array}$ & $\begin{array}{l}\text { GGGAAGGCTGAGGTGATTCT } \\
\text { GTCCCCTTTCTGTCTCAGCT }\end{array}$ \\
\hline P2ry2 & NM_008773.3 & $\begin{array}{l}\text { Forward } \\
\text { Reverse }\end{array}$ & $\begin{array}{l}\text { ATCCTCACCACCTCAAGAGC } \\
\text { GGCAACAGCACGTACTTGAA }\end{array}$ \\
\hline Tnrc18 & NM_001122730.2 & $\begin{array}{l}\text { Forward } \\
\text { Reverse }\end{array}$ & $\begin{array}{l}\text { TCCATCCTACCTCCACCTGA } \\
\text { GGTGCGGGCAGGTAGAAG }\end{array}$ \\
\hline Tgfb3 & NM_009368.3 & $\begin{array}{l}\text { Forward } \\
\text { Reverse }\end{array}$ & $\begin{array}{l}\text { AGGAGACCTCGGAGTCTGAG } \\
\text { CACTGAGGACACATTGAAACGA }\end{array}$ \\
\hline Vcp & NM_009503.4 & $\begin{array}{l}\text { Forward } \\
\text { Reverse }\end{array}$ & $\begin{array}{l}\text { CGAGTTCGCCTAGGAGATGT } \\
\text { AGAGATTGCCAGTGATGCCT }\end{array}$ \\
\hline Ankib1 & NM_001289527.1 & $\begin{array}{l}\text { Forward } \\
\text { Reverse }\end{array}$ & $\begin{array}{l}\text { CCTATCAGCACAACACACCG } \\
\text { TGTTTCATTGTGCACATTCCG }\end{array}$ \\
\hline Panx1 & NM_019482.2 & $\begin{array}{l}\text { Forward } \\
\text { Reverse }\end{array}$ & $\begin{array}{l}\text { TCACATGTATTGCCGTGGGT } \\
\text { CTCGGGGAGAAGCAGCTTAT }\end{array}$ \\
\hline Cacna2d3 & NM_009785.1 & $\begin{array}{l}\text { Forward } \\
\text { Reverse }\end{array}$ & $\begin{array}{l}\text { TGCCTGTGAACATCAGTCTGA } \\
\text { GCCATATGAGAGACGGATCCC }\end{array}$ \\
\hline Fbln2 & NM_007992.2 & $\begin{array}{l}\text { Forward } \\
\text { Reverse }\end{array}$ & $\begin{array}{l}\text { GGGCACTCACGATTGTAGCT } \\
\text { GCAGGTCTGTCACACACTCA }\end{array}$ \\
\hline Grm5 & NM_001081414.2 & $\begin{array}{l}\text { Forward } \\
\text { Reverse }\end{array}$ & $\begin{array}{l}\text { GAGGGTTGTACCTTCGGATG } \\
\text { AAATCCCTTCCTTCGCTGAC }\end{array}$ \\
\hline Chl1 & NM_007697.2 & $\begin{array}{l}\text { Forward } \\
\text { Reverse }\end{array}$ & $\begin{array}{l}\text { AGCAGCTGAAATACCACTCTC } \\
\text { GGGTTTCCTTTCGCTTCACA }\end{array}$ \\
\hline Col2a1 & NM_001113515.2 & $\begin{array}{l}\text { Forward } \\
\text { Reverse }\end{array}$ & $\begin{array}{l}\text { GTGAGCCATGATCCGCCTC } \\
\text { GGTTCTCCTTTCTGCCCCTT }\end{array}$ \\
\hline Gap43 & NM_008083.2 & Forward & GGAAGAAGGCAGGGGAAGAT \\
\hline
\end{tabular}




\begin{tabular}{|l|l|l|l|}
\hline & & Reverse & CTGAATTTTGGTCGCAGCCT \\
\hline Lynx1 & NM_011838.4 & $\begin{array}{l}\text { Forward } \\
\text { Reverse }\end{array}$ & $\begin{array}{l}\text { TCATGACCCATCTGCTCACA } \\
\text { GTGGTCATACAGTAGGTGGC }\end{array}$ \\
\hline Padi2 & NM_008509.2 & $\begin{array}{l}\text { Forward } \\
\text { Reverse }\end{array}$ & $\begin{array}{l}\text { GAGATGGAGAGCAAAGCCCT } \\
\text { GTCTTCAGGGGTCCTTAGGG }\end{array}$ \\
\hline P2rx7 & NM_008812.2 & $\begin{array}{l}\text { Forward } \\
\text { Reverse }\end{array}$ & $\begin{array}{l}\text { GGGAAAATATGCTGCGGGAAC } \\
\text { CACCTTCCGAGTGCTTCAGG }\end{array}$ \\
\hline Pcdh17 & NM_011027.3 & $\begin{array}{l}\text { Forward } \\
\text { Reverse }\end{array}$ & $\begin{array}{l}\text { ACTGCAGACTACACCTTCCC } \\
\text { TACAACGCCGGTCAGAAGAG }\end{array}$ \\
\hline Samd4 & NM_001013753.2 & $\begin{array}{l}\text { Forward } \\
\text { Reverse }\end{array}$ & $\begin{array}{l}\text { CTAATTCGAGCGAGACCCCT } \\
\text { CTCTCAGGCTGGCTCTTTCT }\end{array}$ \\
\hline Co14a6 & NM_001037221.2 & $\begin{array}{l}\text { Forward } \\
\text { Reverse }\end{array}$ & $\begin{array}{l}\text { TTGTGCCTCAGTATGACGGA } \\
\text { AACCCTGAAGATGGCTGACA }\end{array}$ \\
\hline S18 & NM_053185.2 & $\begin{array}{l}\text { Forward } \\
\text { Reverse }\end{array}$ & $\begin{array}{l}\text { CAAGCATGCACCCTGGATTG } \\
\text { AGACTCCATGGCAATCTCGG }\end{array}$ \\
\hline GAPDH & NM_213557 & $\begin{array}{l}\text { Forward } \\
\text { Reverse }\end{array}$ & $\begin{array}{l}\text { TGCGAGTACTCAACACCAACA } \\
\text { CTGCTTTCCTCAACACCACA }\end{array}$ \\
\hline RP2a & NM_017008 & $\begin{array}{l}\text { Forward } \\
\text { Reverse }\end{array}$ & $\begin{array}{l}\text { CAAGGTCATCCATGACAACTTTG } \\
\text { GGGCCATCCACAGTCTTCTG }\end{array}$ \\
\hline
\end{tabular}




\subsection{Common significant gene analysis}

Given a statistical criterion for significance assessment and a dataset $\mathrm{X}$ featuring $\mathrm{x}_{\mathrm{s}}$ significantly regulated genes, one might be interest in comparing it with another dataset $Y$ (containing at least each of the $x_{\mathrm{s}}$ genes) in order to find out how many of those $\mathrm{x}_{\mathrm{s}}$ genes are also significantly regulated in $\mathrm{Y}$ and how much this overlap is likely to be random, rather than representative of a meaningful common transcriptional pattern.

We say:

- $\mathrm{x}_{\mathrm{up}}$ and $\mathrm{x}_{\text {down }}$ the number of genes significantly up- and down-regulated in the dataset $\mathrm{X}$, being $\mathrm{x}_{\mathrm{s}}=\mathrm{x}_{\mathrm{up}}+\mathrm{x}_{\text {down }}$ the total number of genes that passed the test for significance assessment within dataset $\mathrm{X}$;

- $\mathrm{y}_{\mathrm{up}}$ and $\mathrm{y}_{\text {down }}$ the number of genes significantly up- and down-regulated in the dataset $\mathrm{Y}$, being $\mathrm{y}_{\mathrm{s}}=\mathrm{y}_{\text {up }}+\mathrm{y}_{\text {down }}$ the total number of genes that passed the test for significance assessment within dataset $\mathrm{Y}$;

- $\mathrm{n}_{\mathrm{y}}$ the total number of genes present in $\mathrm{Y}$ dataset; it will be necessarily $\mathrm{n}_{\mathrm{y}} \geq \mathrm{y}_{\mathrm{s}}$, even if typically is $\mathrm{n}_{\mathrm{y}}>>\mathrm{y}_{\mathrm{s}}$;

- $\mathbf{E}[z]$ the expectation value of the discrete random variable $\mathrm{z}$.

In this problem we can have basically 6 categories of genes:

\begin{tabular}{|l|l|l|}
\hline$U U$ & $U 0$ & $U D$ \\
\hline$D D$ & $D 0$ & $D U$ \\
\hline
\end{tabular}

- $U U=$ experimental number of genes that are significantly up-regulated in $\mathrm{X}$ and significantly up-regulated in Y (upward co-regulation);

- $D D=$ experimental number of genes that are significantly down-regulated in $\mathrm{X}$ and significantly down-regulated in Y (downward co-regulation);

- $U 0=$ experimental number of genes that are significantly up-regulated in X but are not significant in Y ("up-to-zero");

- $D O=$ experimental number of genes that are significantly down-regulated in $\mathrm{X}$ but are not significant in Y (“down-to-zero");

- $U D=$ experimental number of genes that are significantly up-regulated in $\mathrm{X}$ and significantly down-regulated in Y ("up-to-down" anti-regulation);

- $D U=$ experimental number of genes that are significantly down-regulated in $\mathrm{X}$ and significantly up-regulated in Y (“down-to-up” anti-regulation).

Notice that the problem we are addressing is not fully symmetrical in that we are not interested in those genes that are not significant in $\mathrm{X}$ (typically $\mathrm{X}$ is the dataset to be studied, while $\mathrm{Y}$ is an auxiliary dataset used as reference/comparison/control).

If $\mathrm{X}$ and $\mathrm{Y}$ are independent (e.g. they describe the transcriptome of two completely unrelated pathologies) there should not be any correlation between them, that is to say that significant genes are randomly distributed in the two datasets (at least mutually). Using the previous notations, assuming that $\mathrm{X}$ and $\mathrm{Y}$ are independent and given the experimental occurrences of significant genes present in the two dataset, we can easily predict how many genes we should expect to find by chance in each one of the 6 categories (lower case is used to indicate a discrete random variable): 
By this way we can define a randomized null model based on the empirical proportions of significant genes in $\mathrm{X}$ and $\mathrm{Y}$, the only assumption being the mutually independence of $\mathrm{X}$ and $\mathrm{Y}$. In order to compare empirical data $\{U U, U 0, U D, D D, D O, D U\}$ to the expected values predicted by the null model, we also need a statistical model describing how the 6 random variables $\{u u, u 0, u d, d d, d 0, d u\}$ distribute around their expected values. Binomial distribution can be used to this purpose: $\operatorname{Pr}(k)=\left(\begin{array}{l}n \\ k\end{array}\right) p^{k}(1-p)^{n-k}$, where $\left(\begin{array}{l}n \\ k\end{array}\right)=\frac{n !}{k !(n-k) !}$ is the binomial coefficient, represents the probability mass function describing the probability of getting exactly $k$ successes in $n$ trials, $p$ being the probability of a single success in a single trial. In our case a success is intended to be one of the 6 possible gene combination, $n$ is $\mathrm{x}_{\text {up }}$ or $\mathrm{x}_{\text {down }}$, while $p$ is equal to $\mathrm{y}_{\mathrm{up}} / \mathrm{n}_{\mathrm{y}}$ rather than $\mathrm{y}_{\text {down }} / \mathrm{n}_{\mathrm{y}}$, depending on the particular combination of interest. So we have:

$$
\begin{aligned}
& \operatorname{Pr}(u u=k)=\left(\begin{array}{c}
x_{u p} \\
k
\end{array}\right)\left(\frac{y_{u p}}{n_{y}}\right)^{k}\left(1-\frac{y_{u p}}{n_{y}}\right)^{x_{u p}-k}, \\
& \operatorname{Pr}(u 0=k)=\left(\begin{array}{c}
x_{u p} \\
k
\end{array}\right)\left(1-\frac{y_{s}}{n_{y}}\right)^{k}\left(\frac{y_{s}}{n_{y}}\right)^{x_{u p}-k}, \\
& \operatorname{Pr}(u d=k)=\left(\begin{array}{c}
x_{u p} \\
k
\end{array}\right)\left(\frac{y_{\text {down }}}{n_{y}}\right)^{k}\left(1-\frac{y_{\text {down }}}{n_{y}}\right)^{x_{u p}-k}, \\
& \operatorname{Pr}(d d=k)=\left(\begin{array}{c}
x_{\text {down }} \\
k
\end{array}\right)\left(\frac{y_{\text {down }}}{n_{y}}\right)^{k}\left(1-\frac{y_{\text {down }}}{n_{y}}\right)^{x_{\text {down }}-k}, \\
& \operatorname{Pr}(d 0=k)=\left(\begin{array}{c}
x_{\text {down }} \\
k
\end{array}\right)\left(1-\frac{y_{s}}{n_{y}}\right)^{k}\left(\frac{y_{s}}{n_{y}}\right)^{x_{\text {down }}-k}, \\
& \operatorname{Pr}(d u=k)=\left(\begin{array}{c}
x_{\text {down }} \\
k
\end{array}\right)\left(\frac{y_{u p}}{n_{y}}\right)^{k}\left(1-\frac{y_{u p}}{n_{y}}\right)^{x_{\text {down }}-k} .
\end{aligned}
$$

Notice that, being $\mathbf{E}[k]=n p$ the mean of a binomially distributed random variable, these probability mass functions are consistent with the expectation values already showed in table.

Actually, in order to estimate the probability of see a particular experimental configuration $\{U U, U 0, U D$, $D D, D 0, D U\}$ under the null hypothesis that $\mathrm{X}$ and $\mathrm{Y}$ are independent, we are more interested in the cumulative distribution functions, rather than probability mass functions. In particular, for the first combination $U U$, we have:

$\operatorname{Pr}(u u \geq U U)=\sum_{k=U U}^{x_{u p}}\left(\begin{array}{c}x_{u p} \\ k\end{array}\right)\left(\frac{y_{u p}}{n_{y}}\right)^{k}\left(1-\frac{y_{u p}}{n_{y}}\right)^{x_{u p}-k}$

Notably, $\operatorname{Pr}(u u \geq U U)$ can be regarded as a $p$-value concerning the enrichment of the entire category containing those genes that are significantly up-regulated in both $\mathrm{X}$ and $\mathrm{Y}$ (upward co-regulation). We call it "categorical $p$-value" (to distinguish it from ordinary $p$-values referred to individual genes) since it answer the question: "How likely is it to have $U U$ (or more) genes upwardly co-regulated, under the hypothesis that significant genes are randomly distributed in the two datasets?" 
While the same can be said for the other "lateral" categories of genes ( $U D, D D$ and $D U$ ), the interest in the "central" categories (those featuring genes that are significantly regulated only in X) concerns their possible impoverishment rather than enrichment. In this case, the statistical significance (categorical $p$-value) can be calculated as follow:

$\operatorname{Pr}(u 0 \leq U 0)=\sum_{k=0}^{U 0}\left(\begin{array}{c}x_{u p} \\ k\end{array}\right)\left(1-\frac{y_{s}}{n_{y}}\right)^{k}\left(\frac{y_{s}}{n_{y}}\right)^{x_{u p}-k}$, and analogously for $D 0$.

Notice that, in this kind of problem, the 6 categories of interest are not mutually independent. In particular, because of the 2 constraints $x_{u p}=u u+u d+u 0$ and $x_{\text {down }}=d d+d u+d 0$ only 4 categories out of 6 are actually independent, and the same applies to the enrichment hypothesis tests. Since each comparative analysis of two datasets implies 4 independent hypothesis tests, we can adjust categorical $p$-values for multiple comparisons according to the usual correction techniques: Adj.p.val $=4 \cdot p$-value (Bonferroni), Adj.p.val $=1-(1-p \text {-value })^{4}$ (Dunn-Šidák), etc.

Incidentally, because of the same two constraints cited above, the expectation values of the central categories could be alternatively computed as:

$\mathrm{E}[u 0]=x_{u p}-\mathrm{E}[u u]-\mathbf{E}[u d]$,

$\mathbf{E}[d 0]=x_{\text {down }}-\mathbf{E}[d d]-\mathbf{E}[d u]$.

Even if the two categories $U O$ and $D O$ can be further explored and biologically validated regardless of their categorical $p$-values (simply because they are in any case the container of all those genes specific for the condition studied by $\mathrm{X}$ respect to $\mathrm{Y}$ ), a significant impoverishment of one of them is suggestive of some kind of correlation between $\mathrm{X}$ and $\mathrm{Y}$ (and hence between the pathologies or treatments they represent). On the contrary, the $p$-values assigned to the lateral categories allow to precisely locate any possible (and statistically significant) overlapping of the two dataset, and further investigations are not justified in the absence of a sufficiently low categorical $p$-value. 
1

2

3

4

5

6

7

8

9

\section{List of differentially expressed genes between non-T ProbeName GeneSymb GeneName}

A_52_P267391 Trim12a tripartite motif-containing 12A

A_55_P1974432 Gm5067 predicted gene 5067

A_55_P2070576 Ccl27a chemokine (C-C motif) ligand 27A

A_55_P2205650 2610507I01Ri RIKEN cDNA 2610507I01 gene

A_55_P2064659 Trim12a tripartite motif-containing 12A

A_55_P2256646 C130078N14 uncharacterized protein C130078l

A_55_P2068731 Gm20878 predicted gene, 20878

A_52_P360330 Map1b microtubule-associated protein 1B

A_55_P2068733 Gm20878

A 55_P2092750 Car9

A_55_P1981756 Vmn2r123

A_51_P349888 Ang2

A_52_P88793 Zfp933

A 52 P238846 Bpnt1

A_51_P288549 Jmjd7

A_66_P130730 Zfp963

A_55_P2112986 Klk1b22

A_55_P2134591 BC049715

A_55_P2097151 Ptchd2

A 51 P277006 Chst8

A 55_P2073965 BC049715

predicted gene, 20878

carbonic anhydrase 9

vomeronasal 2, receptor 123

angiogenin, ribonuclease $A$ family

zinc finger protein 933

bisphosphate 3'-nucleotidase 1

jumonji domain containing 7

zinc finger protein 963

kallikrein 1-related peptidase b22 cDNA sequence BC049715

patched domain containing 2

carbohydrate ( $\mathrm{N}$-acetylgalactosam cDNA sequence BC049715

A_51_P217498 Slc2a4

A_55_P2045886 Stk3

A_55_P2180869 Ocel1

solute carrier family 2 (facilitated $\mathrm{g}$ serine/threonine kinase 3 occludin/ELL domain containing 1 A_55_P2108773 4930427A07RRIKEN cDNA 4930427A07 gene A_66_P105689 Trim34a tripartite motif-containing 34A A_66_P134474 Ang3 angiogenin, ribonuclease A family A_55_P1964628 LOC1026336ء tropomyosin alpha-4 chain-like A 51_P293069 Mfsd7b major facilitator superfamily domai A_55_P2085142 Spp1 secreted phosphoprotein 1 A_55_P2154387 Bmp4 bone morphogenetic protein 4 A_51_P413147 Klk1b3 kallikrein 1-related peptidase b3 A_51_P112627 St6galnac2 ST6 (alpha-N-acetyl-neuraminyl-2 A_51_P512820 Dera A_55_P2095603 Ccdc65

A_51_P477121 Pmaip1

A 55 P2130129 Kcnab1 2-deoxyribose-5-phosphate aldola coiled-coil domain containing 65 phorbol-12-myristate-13-acetate-ir potassium voltage-gated channel,

A_55_P2373852 2310058N22FRIKEN cDNA 2310058N22 gene

A_55_P2144597 9030025P20RRIKEN cDNA 9030025P20 gene A_51_P180724 Mlh1 mutL homolog 1 (E. coli)

A_51_P142896 Cd59a CD59a antigen

A_55_P2003561 Luzp2

A_51_P417321 Zfp236

A 51_P115953 Ctxn3

A_55_P2068723 Ccl27a

A_51_P451458 Mamdc2

leucine zipper protein 2

zinc finger protein 236

cortexin 3

chemokine (C-C motif) ligand 27A MAM domain containing 2

A_55_P2068734 Ccl27a

A_55_P2015912 Zfp961

A_55_P1961395 Pdpn

A_51_P358722 Lancl3 chemokine (C-C motif) ligand 27A zinc finger protein 961 podoplanin LanC lantibiotic synthetase compc A 55 P2053551 Mast4 microtubule associated serine/thre A_55_P2019577 1500011B03RRIKEN cDNA 1500011B03 gene A_52_P532687 Ermard ER membrane associated RNA d $\epsilon$ A_52_P209484 Tmem88 transmembrane protein 88 A_52_P490207 Ermard A_55_P2059357 Myo7a

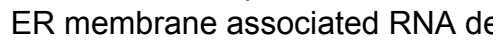
myosin VIIA 

A_51_P507899 Ttc8
A_55_P2194064 BC023969
A 55 P2067727 Mxra7
A 55 P2197338 Tnfsf13os
A_51_P345316 Cep76
A_55_P2065726 Snx29
A_55_P2179793
A_52_P642012
A_52_P616332
A 51P179504 Ang3
A 55P2169963 Gm13152
A_52_P559545 Cercam
A_55_P2180196 Ccdc32
A_52_P135707 Creb3
tetratricopeptide repeat domain 8 cDNA sequence BC023969
matrix-remodelling associated 7
tumor necrosis factor (ligand) supe
centrosomal protein 76
sorting nexin 29
0
cDNA sequence BC006965
ATPase, class $V$, type 10D
angiogenin, ribonuclease $A$ family predicted gene 13152
cerebral endothelial cell adhesion coiled-coil domain containing 32
cAMP responsive element binding

A 52 P 598634

A_52_P587738

A 55_P2380428 5430416G10

A 66 P122158 Pisd-ps3

A 52 P592909 Dgat2

A_52_P549977 Fam32a

A_51_P125368 Hars

A_55_P2019054 Acacb

A_55_P1987196 Gm3448

A_55_P1960167 Bcat2

A 51_P480013 Car11

A_55_P2137611 Irgm2

A_55_P2120866 Gm7120

A_51_P418908 Larp1

A_55_P1975874 Bcl2l15

A_51_P286814 Ncor2

A_55_P2108883 AV356131

A_51_P155458 Dok7

A 55 P2054350 Fbxo44

A_55_P2083213 Purb

A_51_P490747 Al593442

A_52_P311853 Ddit4I

A_55_P2065059 Wnt2

A_55_P2056325 Anxa3

A 55 P1998401 Eif2ak4

A_55_P2079158 Fam154b

A_55_P2153941 Zfp386

A_51_P237383 Rnase4

A_55_P1974602 Map2k7

A_51_P155174 Zfp672

A_55_P2097156 Ptchd2

A_52_P295104 Smim5

A_55_P2151138 Dynlt1f

A 55 P2201612 Slc30a7

A_55_P2109544 Trnt1

A 66 P120770 Ywhaz

A_55_P1983268

A_51_P191520

A_66_P130366

A 55 P2018330 Gm13298

A_55_P2005055 Pepd

A_51_P470989 Paip1

A_55_P2127587 Smcr8

A_55_P1972490 Psg16 RIKEN cDNA 1190007107 gene purinergic receptor P2Y, G-proteir RIKEN cDNA 5430416G10 gene phosphatidylserine decarboxylase diacylglycerol O-acyltransferase 2 family with sequence similarity 32 , histidyl-tRNA synthetase acetyl-Coenzyme A carboxylase b predicted gene 3448 branched chain aminotransferase carbonic anhydrase 11 immunity-related GTPase family N predicted gene 7120

La ribonucleoprotein domain famil BCLI2-like 15

nuclear receptor co-repressor 2 expressed sequence AV356131 docking protein 7

F-box protein 44

purine rich element binding proteir expressed sequence Al593442

DNA-damage-inducible transcript wingless-related MMTV integratior annexin A3

eukaryotic translation initiation fac family with sequence similarity 15 , zinc finger protein 386 (Kruppel-lik ribonuclease, RNase A family 4 mitogen-activated protein kinase $k$ zinc finger protein 672

patched domain containing 2 small integral membrane protein 5 dynein light chain Tctex-type $1 \mathrm{~F}$ solute carrier family 30 (zinc trans: tRNA nucleotidyl transferase, CCF tyrosine 3-monooxygenase/tryptor 930444P10R RIKEN cDNA 4930444P10 gene START domain containing 10 serine/threonine kinase 38 like RIKEN cDNA $1700001 \mathrm{C} 19$ gene predicted gene 13298 peptidase $D$ polyadenylate binding protein-inte Smith-Magenis syndrome chromo: pregnancy specific glycoprotein $1 \epsilon$ 
1

2

3

4

5

6

7

8

9 growth factor receptor bound prote predicted gene 10845

ribosomal protein $\mathrm{L} 15$ cDNA sequence BC026762 dedicator of cytokinesis 4 retinal degeneration 3 A_51_P494863 Vmac vimentin-type intermediate filamer A_55_P2149382 Gm3448 predicted gene 3448 A 55 P2221647 Al605517 expressed sequence Al605517 A 51_P140237 Fhl2 four and a half LIM domains 2 A_55_P1989524 Fndc1 fibronectin type III domain contain A 51 P159293 Zbbx A_55_P1995924 Gm13157

A_51_P452820 Rpl31

A_52_P123738 Rnf41

A_52_P222230

A_51_P327564 Glb1

A_51_P422335 Zfp420

A_55_P2146749 Rps13

A_66_P116461 Mro

A_51_P375558 Myoc

A_51_P123604 Ppwd1

A_55_P1962756 Ttll2

A_51_P316801 Wdr60

A_51_P228193 Ociad1

A_55_P1998995 Speg

A_52_P489778 Ablim1

A_52_P512553 Atg1612

A_65_P08864 Dph5

A_55_P1985428 Atg1612

A_52_P325477 Trim16

A_66_P108434 Ccdc65

A_51_P502150 Slc9a3r1

A 55 P1970033 Per1

A_51_P409893 Prkar2a

A_55_P2161465 Gm10516

A_55_P2031547 Vmn2r121

A_55_P2062133 Etv3

A_51_P314153 Nr2c2ap

A_55_P1967539 Hunk

A_51_P140607 Asun

A_55_P2012694 Kcnh5

A_51_P209183 Cxcl14

A_52_P253179 lgfbp3

A_55_P1968977 Stk38I

A_55_P1982454 Eps8

zinc finger, B-box domain containi predicted gene 13157

ribosomal protein L31

ring finger protein 41

0

galactosidase, beta 1

zinc finger protein 420

ribosomal protein $\mathrm{S} 13$

maestro

myocilin

peptidylprolyl isomerase domain a tubulin tyrosine ligase-like family, I WD repeat domain 60

OCIA domain containing 1

SPEG complex locus

actin-binding LIM protein 1

autophagy related 16 -like 2 (S. ce DPH5 homolog (S. cerevisiae) autophagy related 16 -like 2 (S. ce tripartite motif-containing 16 coiled-coil domain containing 65 solute carrier family 9 (sodium/hyc period circadian clock 1

protein kinase, cAMP dependent $r$ predicted gene 10516

vomeronasal 2, receptor 121

ets variant gene 3

nuclear receptor $2 \mathrm{C} 2$-associated $\mathrm{r}$ hormonally upregulated Neu-asso asunder, spermatogenesis regulat potassium voltage-gated channel, chemokine (C-X-C motif) ligand $1 /$ insulin-like growth factor binding $p$ serine/threonine kinase 38 like epidermal growth factor receptor $\mathrm{F}$ A_55_P2000007 LOC1026393: uncharacterized LOC102639358 A_51_P481821 Spcs3 signal peptidase complex subunit A_51_P480202 Dlx2

A_55_P2127258 Dok7

A_55_P2062688 Msi1

A_55_P2023697 Zfp386

A_51_P320022 Atp10a

A_51_P356579 Mxra7

A_55_P1967514 Dnah7a

A_52_P217710 Fzd6

A_55_P2036723 Stk36

A_55_P1978226 Park2 distal-less homeobox 2

docking protein 7

musashi RNA-binding protein 1 zinc finger protein 386 (Kruppel-lik ATPase, class $V$, type $10 \mathrm{~A}$ matrix-remodelling associated 7 dynein, axonemal, heavy chain $7 \mathrm{~A}$ frizzled homolog 6 (Drosophila) serine/threonine kinase 36 Parkinson disease (autosomal rec 
A 55 P1999958

0

A_66_P112301 C230072F16FRIKEN cDNA C230072F16 gene

A_51_P244824 Dapp1 dual adaptor for phosphotyrosine :

A_55_P1969431 Lyrm5 LYR motif containing 5

A_55_P2145521 Stk38I serine/threonine kinase 38 like

A_55_P2259125 D7Ertd143e DNA segment, Chr 7, ERATO Doi

A_55_P2206605 5830444B04RRIKEN cDNA 5830444B04 gene

A_55_P2151143 Dynlt1c dynein light chain Tctex-type 1C

A_51_P333965 Cisd2

A 52 P2710 Cml5

A 55P2176248

CDGSH iron sulfur domain 2

A 52 P456561 Abcd1

camello-like 5

A_55_P2183735 Pisd

A_52_P565279 Cecr5

A_51_P448391 Nkiras1

A_55_P1985693 Fhdc1

A_51_P269634 Zfp14

A_55_P2033480 Gm13298

0

ATP-binding cassette, sub-family I phosphatidylserine decarboxylase cat eye syndrome chromosome re NFKB inhibitor interacting Ras-lik $\epsilon$ $\mathrm{FH} 2$ domain containing 1 zinc finger protein 14 predicted gene 13298

A 55 P2170509 Yipf4 Yip1 domain family, member 4

A 51 P143468 KIhl26 kelch-like 26

A_52_P436590 Wbscr17

A_52_P547589 Spag1

A_55_P1990134 Cox18

A_55_P2002226 Dzip1

A_66_P101108 Tnrc18

A_55_P1987151 Nlrp5

A_55_P1988882 Sept9

Williams-Beuren syndrome chrom sperm associated antigen 1 cytochrome $c$ oxidase assembly $p$ DAZ interacting protein 1 trinucleotide repeat containing 18 NLR family, pyrin domain containiı septin 9

A_55_P2144280 Nnt

A_55_P2255737

A_55_P2045114 Tmem242 nicotinamide nucleotide transhydrı

A_55_P2186558 Tmem242

0

A_55_P2212498 C030005K06F RIKEN cDNA C030005K06 gene

A_55_P2067652 Boc biregional cell adhesion molecule-

A_55_P1995992 Gm14432 predicted gene 14432

A_51_P263246 Dusp8 dual specificity phosphatase 8

A_51_P433091 Purb

A_52_P106620 Tnfrsf11b

A_51_P324934 Mcm3

A_55_P2049211 Pisd-ps1

A_51_P193302 Mrps7

A_51_P342707 Pold2

A_55_P2163729 Tvp23a

A_55_P2085333 Fbxo44

purine rich element binding proteir tumor necrosis factor receptor sup minichromosome maintenance de phosphatidylserine decarboxylase mitchondrial ribosomal protein S7 polymerase (DNA directed), delta trans-golgi network vesicle protein F-box protein 44

A_55_P1967820 Al661453

A_55_P1980292 Purb

A_55_P1964638 Cxadr

A_55_P1956918 Adamts5

A_55_P2037883 Ino80

A_55_P2173927 Insr expressed sequence Al661453 purine rich element binding proteir coxsackie virus and adenovirus re a disintegrin-like and metallopeptic INO80 homolog (S. cerevisiae) insulin receptor

A_51_P487913 2600006K01RRIKEN cDNA 2600006K01 gene A_55_P2028370 0

A_55_P1991851 Speg

A_55_P2144285 Nnt

A_55_P1955568 Ext12

SPEG complex locus

A_55_P1976993 Strn

A_51_P475228 Armc6

A_55_P2140118 Qpct

A_52_P260696 Arnt2 nicotinamide nucleotide transhydri exostoses (multiple)-like 2 striatin, calmodulin binding protein armadillo repeat containing 6 glutaminyl-peptide cyclotransferas aryl hydrocarbon receptor nuclear 
1

2

3

4

5

6

7

8

9

\begin{tabular}{|c|c|c|}
\hline _52_P627068 & Disp2 & dispatched homolog 2 (Drosophila \\
\hline _55_P2211937 & E130101M22 & uncharacterized protein E130101/ \\
\hline _55_P2045658 & Nme6 & NME/NM23 nucleoside diphospha \\
\hline _55_P2322029 & $3830406 \mathrm{C} 13 \mathrm{~F}$ & RIKEN cDNA 3830406C13 gene \\
\hline 55_P1979929 & Prcp & prolylcarboxypeptidase (angiotens \\
\hline _52_P646979 & D16Ertd472e & DNA segment, Chr 16 , ERATO Dc \\
\hline _55_P2146500 & Ccdc107 & coiled-coil domain containing 107 \\
\hline _51_P127915 & Rnasek & ase, RNase $\mathrm{K}$ \\
\hline _51_P383629 & Vps4 & vacuolar protein sorting \\
\hline _52_P67200 & Stt3b & STT3, subunit of the olig \\
\hline A_55_P2026818 & Slc4a7 & solute carrier fam \\
\hline 55_P1955548 & Ezr & ezrin \\
\hline 55_P1995874 & Gm14326 & predicted gen \\
\hline _55_P2122633 & Airn & antisens \\
\hline A_66_P110742 & 0 & 0 \\
\hline _51_P414548 & Casp7 & caspase 7 \\
\hline _55_P2035717 & Pgap2 & post-GPI attachment to proteins \\
\hline _55_P197 & Stard6 & elated lipid transfer (STAR1 \\
\hline I55_P204 & Zfp74 & \\
\hline _55_P1979330 & Dapp1 & osphotyrosine : \\
\hline A_55_P19 & Zfp868 & \\
\hline A_52_P14526 & Zyg & cycle \\
\hline A_52_P484838 & Rfxank & or X-associated ank \\
\hline A_52_P604629 & Csrnp1 & ch nuclear protei \\
\hline A_55_P2161695 & Kdelc1 & KDEL (Lys-Asp-Glu-Leu) containir \\
\hline A_51_P102507 & Vps33a & sorting 33A (yeas \\
\hline A_55_P200 & Ccdc144b & ain containing $144 \mathrm{E}$ \\
\hline A_55_P20 & Atat1 & |ltransferase 1 \\
\hline A_51_P3 & Oxsm & CP synthase, $\mathrm{m}$ \\
\hline A_55_P2 & Gyk & \\
\hline A_55_P2 & D17Zt10e & DN \\
\hline A_66_P & Net1 & ng gi \\
\hline A_52_P2 & D16Ertd472e & DNA \\
\hline A_51_P48 & Kcnk2 & , subfa \\
\hline A_51_P233 & Rmdn3 & icrotubule dynamics \\
\hline A_51_P31C & Galr2 & \\
\hline A_51_P119031 & Naa30 & $\mathrm{N}($ alp \\
\hline A_55_P21 & Arhgef39 & otide exchange \\
\hline A_55_P & Stac & and cys \\
\hline A_b1. & Psca & intigen \\
\hline A_51 & Tex33 & \\
\hline & 0 & \\
\hline A_55_P20 & Frmd4a & FEF \\
\hline A_55_P2 & Tubd1 & \\
\hline A_51_P18 & Rbks & ribo \\
\hline A_51_P280532 & Supt16 & supp \\
\hline A_55_P21 & Zfp882 & \\
\hline A_55_P19 & Ntng1 & \\
\hline A_52_P6 & Dhrsx & ductase (SDR fi \\
\hline A_66_P138 & 4833419G08F & FIKEN cDNA 4833419G08 gene \\
\hline A_55_P199 & Trim16 & tripa \\
\hline A_55_P199 & Emx2os & opposite strand/antisense tr \\
\hline & & \\
\hline _52_P1187949 & Trim5 & \\
\hline A_51_P367780 & Adamtsl2 & ADA \\
\hline A_55_P2146996 & Wdr52 & \\
\hline A_51_P14 & & peta (A4) precursor protei \\
\hline A_55_P2173( & & \\
\hline A_51_P2832 & Gm14326 & predicted gene 14326 \\
\hline
\end{tabular}



A_52_P376360 Pdgfc
A_55_P1954436 Gm7967
platelet-derived growth factor, $\mathrm{C} \mathrm{p}$ predicted gene 7967
A_55_P1988424 Tpi1
A 65-P02321 Usp36
A_51_P343851 Tgfbrap1
triosephosphate isomerase 1
ubiquitin specific peptidase 36
transforming growth factor, beta $\mathrm{r} \epsilon$
A_55_P1967538 Hunk
A_51_P246727 Mlxip
hormonally upregulated Neu-asso
MLX interacting protein
A_55_P2187043 Tpm1 tropomyosin 1, alpha
A_51_P454103 Manba
mannosidase, beta A, lysosomal
A 52_P494514 Insr
A_55_P2057994 Mif4gd
insulin receptor
A_55_P2007243 Kcnc1
A_51_P441494 BC100451
MIF4G domain containing potassium voltage gated channel, cDNA sequence BC100451
A_52_P74576 Ccdc65
coiled-coil domain containing 65
A_55_P1993876 Otud7a
OTU domain containing $7 \mathrm{~A}$
A_51_P382928 Cstf3
cleavage stimulation factor, 3 ' pre-
A_52_P259558 Ogfod1 2-oxoglutarate and iron-dependen
A_55_P2037081 2610305D13FRIKEN cDNA 2610305D13 gene
A_52_P412529 Fbxo3 F-box protein 3
A_51_P403636 Smad7
SMAD family member 7
A_55_P2062469 Col12a1
collagen, type XII, alpha 1
A_55_P1992715 lgfbp3
insulin-like growth factor binding $p$
A_55_P2113356 Miip
A_55_P2060278 Fam45a migration and invasion inhibitory $p$
A_55_P2039556 Pak6 family with sequence similarity 45 , A 55 P2033445 Tnfrsf1b tumor necrosis factor receptor sup A_51_P308029 2010107G23F RIKEN cDNA 2010107G23 gene A_55_P2034600 Gm5523 glyceraldehyde-3-phosphate dehy A_55_P2054300 Alg8 asparagine-linked glycosylation 8 A_52_P563617 Ssbp4 single stranded DNA binding prot $\epsilon$ A_55_P2241204 1500015A07RRIKEN cDNA 1500015A07 gene A_51_P124748 Tgfb3 transforming growth factor, beta 3 A_66_P113662 Tmem62 transmembrane protein 62
A_55_P1985554 B4galt4 UDP-Gal:betaGIcNAc beta 1,4-ga A_55_P2148641 Rab4a RAB4A, member RAS oncogene $f$ A_55_P1996086 Gm14325 predicted gene 14325
A_55_P1966958 Mef2d
A_52_P144794 Tceanc2 transcription elongation factor A (ई A_55_P2427685 Agl amylo-1,6-glucosidase, 4-alpha-gl A_55_P1969166 Fxr2 fragile $X$ mental retardation, autos A_55_P1973447 $\mathrm{Ybx} 2 \quad \mathrm{Y}$ box protein 2 A_55_P2052563 Id1 inhibitor of DNA binding 1 A_55_P2078213 1700020I14Ri RIKEN cDNA 1700020114 gene A_52_P110068 Rqcd1 rcd1 (required for cell differentiatic A_55_P2147791 Fam129c family with sequence similarity 12? A_55_P2026109 Rpap1 RNA polymerase II associated prc A_55_P2354336 0
A_66_P125212 Mapk1ip1I mitogen-activated protein kinase 1 A_55_P2076927 Ints10 integrator complex subunit 10 A_52_P558382 Mapk8 mitogen-activated protein kinase $\varepsilon$ A_55_P2030721 Ankle1 A_55_P2006525 AdamtsI4 A_52_P505143 Jrk A_51_P144648 Vps13b
A_55_P2147457 Gm6410
A_55_P2013396 Gm14305
A_55_P2172566 Insr A_52_P137691 Trappc5 A_55_P2187038 Tpm1 ankyrin repeat and LEM domain $\mathrm{C}$ ADAMTS-like 4 jerky vacuolar protein sorting 13B (yeas predicted gene 6410 predicted gene 14305 insulin receptor trafficking protein particle complex tropomyosin 1 , alpha 
1

2

3

4

5

6

7

8

9

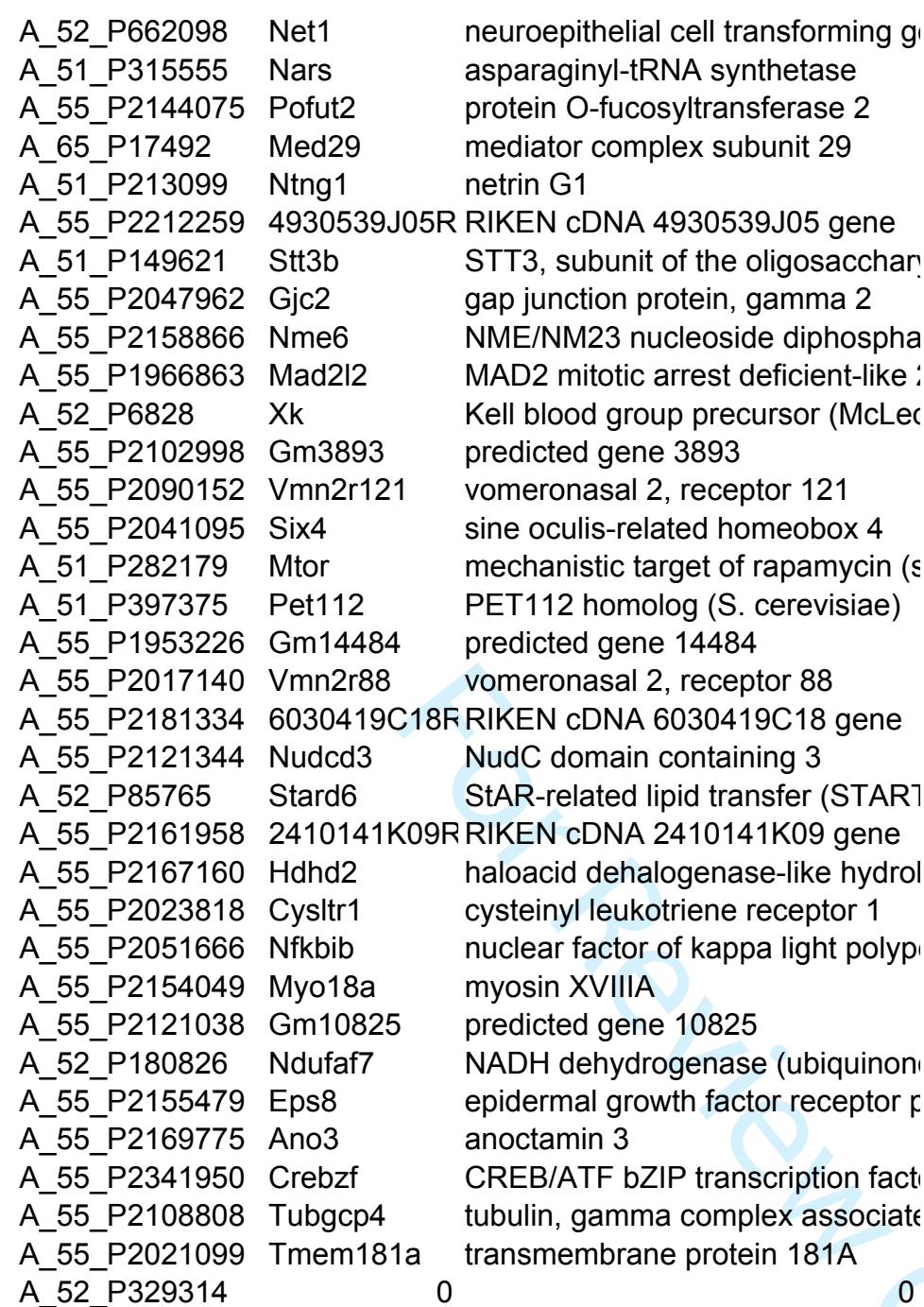

A_55_P2015715 Rab4a

A_55_P2266880 Kirrel

A_51_P215190 Efcab11

A_51_P517381 Cers2

A_55_P2134645 Fam227a

A_55_P2115995 Sms

A_51_P211341 Eif3j1

A_55_P1998194 Snrpn

A_52_P65494 Iqgap2

A_52_P404895 Tmem62

A_55_P2042146 Fech

A_55_P2081398

A_51_P221132 L2hgdh

A_55_P1974442 Sumf2

RAB4A, member RAS oncogene $f$ kin of IRRE like (Drosophila)

EF-hand calcium binding domain . ceramide synthase 2

family with sequence similarity 22 ; spermine synthase

eukaryotic translation initiation fac small nuclear ribonucleoprotein $\mathrm{N}$ IQ motif containing GTPase activa transmembrane protein 62 ferrochelatase

0 L-2-hydroxyglutarate dehydrogene sulfatase modifying factor 2

A_55_P2105563 1700104L18R RIKEN cDNA 1700104L18 gene A_51_P257684 Stau1 staufen (RNA binding protein) hon A_52_P412452 Cntn6 contactin 6

A_55_P2187171 Sv2c synaptic vesicle glycoprotein 2c

A_55_P1961645 Vcp valosin containing protein

A_55_P1955172 Camk2d calcium/calmodulin-dependent prc

A_55_P2433218 Bloc1s6 biogenesis of lysosomal organelle

A_51_P292368 Tmco6 transmembrane and coiled-coil do

A_55_P2168118 4933406C10FRIKEN cDNA 4933406C10 gene

A_52_P363216 Gcnt2 glucosaminyl (N-acetyl) transferas

A_55_P2094881 Fgfr2 fibroblast growth factor receptor 2 
A_55_P2326337 BC026513 cDNA sequence BC026513

A_66_P111216 4632427E13RRIKEN cDNA 4632427E13 gene

A_55_P2133165 Wwc1 WW, C2 and coiled-coil domain cc

A_55_P2086983 Atxn2 ataxin 2

A_55_P1962174 Rab8a RAB8A, member RAS oncogene $f$

A_55_P2018681 G630016G05F RIKEN cDNA G630016G05 gene

A_52_P417990 Zfp868 zinc finger protein 868

A_51_P125183 Coq5 coenzyme Q5 homolog, methyltra

A_55_P2261772 Lzts1 leucine zipper, putative tumor sup

A_55_P2163363 Clec2f C-type lectin domain family 2, mer

A_55_P1998392 Eif2ak4 eukaryotic translation initiation fac

A_55_P2098305 Coq5 coenzyme Q5 homolog, methyltra

A_55_P2032478 Sfxn5 sideroflexin 5

A_55_P2092776 Apoo apolipoprotein O

A_55_P2012241 Ctso cathepsin O

A_55_P2452384 Mga MAX gene associated

A_52_P663526 Nmrk1 nicotinamide riboside kinase 1

A_55_P1974063 Gm2545 predicted gene 2545

A_55_P2050592 Gm5785 predicted gene 5785

A_51_P411728 2900026A02RRIKEN cDNA 2900026A02 gene

A_55_P1970578 Aamdc adipogenesis associated Mth938 c

A_52_P299358 Lclat1 lysocardiolipin acyltransferase 1

A_55_P2288285 2310007J06R RIKEN cDNA 2310007J06 gene

A_55_P2115968 Gm14325 predicted gene 14325

A_51_P336391 Tmem18 transmembrane protein 18

A_52_P67983 Lcmt2 leucine carboxyl methyltransferast

A_51_P114456 Tceanc2 transcription elongation factor A (

A_55_P1962384 Churc1 churchill domain containing 1

A_55_P2053206 Kdm4c lysine (K)-specific demethylase 4C

A_55_P1979246 Cep192 centrosomal protein 192

A_51_P341010 Ercc8 excision repaiross-complementing

A_55_P2388687 1700003G18FRIKEN cDNA 1700003G18 gene

A_55_P2043430 00

A_55_P2078675 00

A_55_P2174273 Slco5a1 solute carrier organic anion transp

A_52_P147666 Slc30a7 solute carrier family 30 (zinc trans

A_55_P2085731 Stk38 serine/threonine kinase 38

A_55_P2160737 0

A_55_P1965674 Alg2 asparagine-linked glycosylation 2

A_55_P2170911 Gm10366 predicted gene 10366

A_52_P425510 Dnah7a dynein, axonemal, heavy chain 7A

A_55_P2104532 Acacb acetyl-Coenzyme A carboxylase b

A_55_P2383283 2310001H17FRIKEN cDNA 2310001H17 gene

A_52_P600946 Ccdc88c coiled-coil domain containing 88C

A_55_P1999883 Gm14499 predicted gene 14499

A_52_P916539 Zbtb34 zinc finger and BTB domain conta

A_66_P107790 Myl12a myosin, light chain 12A, regulator)

A_55_P2045535 Rsg1 REM2 and RAB-like small GTPasi

A_55_P1973896 Vmn2r86 vomeronasal 2, receptor 86

A_55_P2012107 Vmn2r10 vomeronasal 2, receptor 10

A_52_P151905 Gm5132 predicted gene 5132

A_55_P2169909 Ostm1 osteopetrosis associated transmeI

A_55_P2186460 Emx2os Emx2 opposite strand/antisense tr

A_51_P381230 Zhx2 zinc fingers and homeoboxes 2

A_55_P2161485 Ptchd2 patched domain containing 2

A_55_P2160910 Faim2 Fas apoptotic inhibitory molecule :

A_55_P1986208 Ccnb1ip1 cyclin B1 interacting protein 1

A_52_P622694 Adal adenosine deaminase-like

A_55_P2293967 Tbc1d5 TBC1 domain family, member 5 
1

2

3

4

5

6

7

8

9

$\begin{array}{lll}\text { A_55_P1953984 } & \text { Gm11033 } & \text { predicted gene 11033 } \\ \text { A_66_P130911 } & \text { Proser2 } & \begin{array}{l}\text { proline and serine rich 2 } \\ \text { citrate lyase beta like }\end{array} \\ \text { A_52_P88818 } & \text { Clybl } & \text { predicted gene 13298 } \\ \text { A_55_P2033481 } & \text { Gm13298 } & \text { CD84 antigen }\end{array}$

A 51 P320357 Grin2b

A_52_P504330 Ankib1

A_51_P394154 Ddx51

A 55 P2055642 $2810407 A$

A 52 P219913 Cdan1 congenital dyserythropoietic anem

A_51_P220150 Angpt17 angiopoietin-like 7

A_51_P228865 Zfp72 zinc finger protein 72

A_55_P1993094 Mesdc1

A_55_P2005853 Nacc2

A_51_P473953 Arhgef26

A_55_P2057247 Etohi1

A_51_P226962 Capn15

A_51_P266774 Mfn2

A_51_P343429 Slc25a37

A_55_P2022434 Gpi1

A_55_P2025363 Ccnl2

A_55_P2030433 Gpi1

A_55_P2002918 Klc2

A_55_P1977649 Hoxd8

A_55_P2031382 Crebzf

A_55_P2124016 Nipsnap1

A_55_P2057268 Magi1

A_55_P2139713 Phf13

A_65_P10433 Rpp14

A_55_P2005680 Ipo11

A_55_P2134351 Lancl3

A_66_P122053 Kcnq5

A_55_P2002220 Dzip1

A_55_P1956687 Rab37

A_52_P221588 Gm5382

A_55_P2062787 Mfap3

A_55_P2094484 Gm14137

A_52_P18765 Hsbp1l1

A_55_P1983999 Desi1

A_51_P495581 TIr1

mesoderm development candidate nucleus accumbens associated 2 , Rho guanine nucleotide exchange ethanol induced 1

calpain 15

mitofusin 2

solute carrier family 25 , member 3 glucose phosphate isomerase 1

cyclin L2

glucose phosphate isomerase 1

kinesin light chain 2

homeobox D8

CREB/ATF bZIP transcription fact 4-nitrophenylphosphatase domain membrane associated guanylate $k$ PHD finger protein 13

ribonuclease $\mathrm{P} 14$ subunit

importin 11

LanC lantibiotic synthetase compc potassium voltage-gated channel,

DAZ interacting protein 1

RAB37, member RAS oncogene $f$ predicted gene 5382

microfibrillar-associated protein 3 predicted gene 14137

heat shock factor binding protein 1 desumoylating isopeptidase 1 toll-like receptor 1

A_55_P2197134 A930018M24F RIKEN cDNA A930018M24 gene A_52_P146403 Arhgef38 Rho guanine nucleotide exchange A_55_P2066219 Gm3455 predicted gene 3455

A_51_P390775 Ube2e1 ubiquitin-conjugating enzyme E2E

A_51_P494006 Scaf8 SR-related CTD-associated factor

A_51_P243134 Adcy6 adenylate cyclase 6

A_55_P1955851 Al593442 expressed sequence Al593442

A_55_P2024555 Ppap2a phosphatidic acid phosphatase tyr

A_55_P2108868 Nufip1 nuclear fragile $X$ mental retardatio

A_51_P375693 Tmem135 transmembrane protein 135

A_55_P1982494 1700012L04R RIKEN cDNA 1700012L04 gene

A_51_P129100 Sec63 SEC63-like (S. cerevisiae)

A_55_P2003228 Rerg RAS-like, estrogen-regulated, groI

A_55_P2060722 Uso1 USO1 vesicle docking factor

A_55_P2052210 Gdi2 guanosine diphosphate (GDP) dis

A_55_P1970887 Vmn2r16 vomeronasal 2, receptor 16

A_55_P2061809 Ndufc2 NADH dehydrogenase (ubiquinon

A_65_P10180 Rad23b RAD23b homolog (S. cerevisiae)

A_55_P2049483 0
RAD23b homolog (S. cerevisiae)

https://mc04.manuscriptcentral.com/cares 
A_52_P328078 Atp5b ATP synthase, $\mathrm{H}+$ transporting $\mathrm{mi}$

A_51_P342669 Pgam1 phosphoglycerate mutase 1

A_55_P2217548 4921515G04FRIKEN cDNA 4921515G04 gene

A_51_P263290 Galnt9 UDP-N-acetyl-alpha-D-galactosan

A_55_P2078815 Xk

A 55 P2107447 Rab37

A_51_P150608 Jagn1

A_52_P580582 Nppa

A_51_P107433 Mrpl34

A_55_P1960621 Gm20764

A_51_P146303 Mvb12a

A_51_P242356 Fam114a2

A_55_P2041372 Gm3693

A_51_P476711 Skiv2l2

A_51_P133747 Ppp1r3e

A_51_P288505 Tradd

A_55_P1968683 Anks1b

A_55_P2177233 Abhd5

A_55_P2071326 Rpl36

A_51_P208377 Trappc5

Kell blood group precursor (McLec RAB37, member RAS oncogene $f$ jagunal homolog 1 (Drosophila)

natriuretic peptide type $A$ mitochondrial ribosomal protein Li predicted gene, 20764 multivesicular body subunit $12 \mathrm{~A}$ family with sequence similarity 11 。 predicted gene 3693

superkiller viralicidic activity 2 -like protein phosphatase 1 , regulatory TNFRSF1A-associated via death , ankyrin repeat and sterile alpha $\mathrm{m}$ abhydrolase domain containing 5 ribosomal protein L36

trafficking protein particle complex

A_52_P133578 Gpr158 G protein-coupled receptor 158

A_51_P289414 Spg11 spastic paraplegia 11

A_55_P2002113 Rpl36 ribosomal protein L36

A_55_P2041893 Gm6404 predicted gene 6404

A_51_P244558 Rab3gap2 RAB3 GTPase activating protein s A_55_P1999829 Thoc7 THO complex 7 homolog (Drosopl A_55_P2143499 Pgbd5 piggyBac transposable element $d \epsilon$ A_55_P2012096 Bmp8a bone morphogenetic protein 8a

A_55_P2058953 Rpl13a

A_55_P2026054 Usp37

A_55_P2027077 Shc2

A 55P2067131 Dclre1c

A_51_P131025 Ngdn

A_55_P1967648 Flcn

A_55_P2044967 Zfp74

A_52_P117576 Casp3

A_51_P184223 Pcdhb7

A_55_P1956418 Efr3b

A_51_P268843 Rasip1

A_51_P421140 Tubb6

A_52_P275678 Gpr135

A_51_P246677 Rec8

A_51_P241943 Sap30I

A_51_P234627 Nubpl

A_55_P2175915 Ccl28

A_52_P47781 Slitrk3

A_55_P1967983 Use1

A_51_P167374 Gpatch1

A_51_P456838 Fbxl21

A_55_P2056186 Siva1

A_55_P2088711 Sgsm1 ribosomal protein L13A ubiquitin specific peptidase 37 SHC (Src homology 2 domain con DNA cross-link repair $1 \mathrm{C}, \mathrm{PSO} 2 \mathrm{~h}$ neuroguidin, EIF4E binding proteir folliculin

zinc finger protein 74

caspase 3

protocadherin beta 7

EFR3 homolog B (S. cerevisiae)

Ras interacting protein 1

tubulin, beta 6 class $V$

$\mathrm{G}$ protein-coupled receptor 135

REC8 meiotic recombination prote

SAP30-like

nucleotide binding protein-like chemokine (C-C motif) ligand 28

SLIT and NTRK-like family, memb unconventional SNARE in the ER $G$ patch domain containing 1 F-box and leucine-rich repeat prot SIVA1, apoptosis-inducing factor small $G$ protein signaling modulatc

A_55_P2057941 1700049G17FRIKEN cDNA 1700049G17 gene

A_52_P561377 Fam160b1 family with sequence similarity 161

A_52_P552589 Map4k1 mitogen-activated protein kinase $k$

A_55_P2104572 6330416G13FRIKEN cDNA 6330416G13 gene

A_52_P108321 Ccdc71 coiled-coil domain containing 71

A_55_P2114863 Mgll

A_55_P2069012 Pidd1 monoglyceride lipase

p53 induced death domain protein 
1

2

3

4

5

6

7

8

9

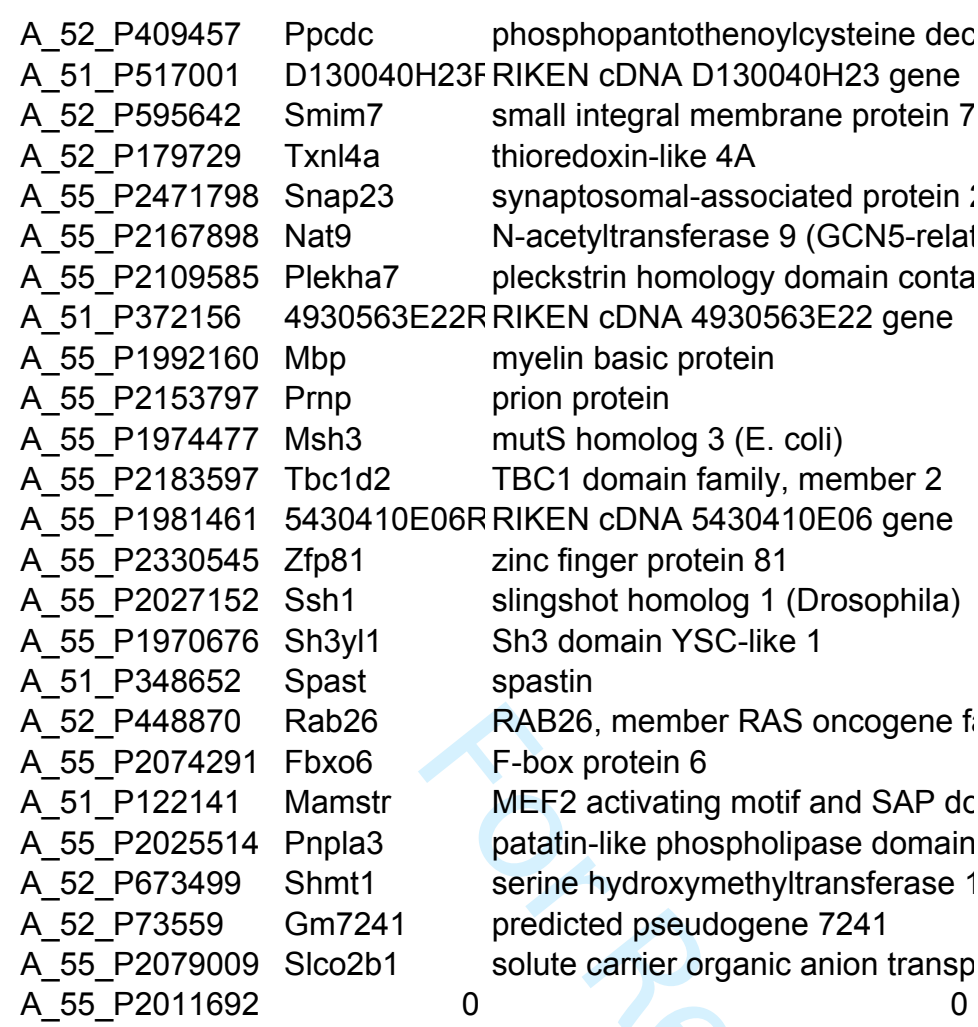

A_55_P1977454 4930570G19FRIKEN cDNA 4930570G19 gene

A_55_P2032147 Wnt9a wingless-type MMTV integration $\mathrm{s}$ A_55_P2003951 Tmem192 transmembrane protein 192

A_52_P734742 Lrrc73 leucine rich repeat containing 73

A_55_P2000027 Spink2 serine peptidase inhibitor, Kazal ty

A_55_P2013203 Oxtr oxytocin receptor

A 51_P291682 Tmed4 transmembrane emp24 protein tra

A_52_P434841 Coa4 cytochrome c oxidase assembly fe

A_55_P2051313 Gstk1 glutathione S-transferase kappa 1

A_55_P2039061 Trim12C tripartite motif-containing 12C

A_52_P327402 Cds1 CDP-diacylglycerol synthase 1

A_55_P2115225 Fap fibroblast activation protein

A_51_P105520 Nomo1 nodal modulator 1

A 55 P2308743 A430106A12FRIKEN cDNA A430106A12 gene

A 55 P2157360 Tagap1 T cell activation GTPase activatinc

A_51_P239286 Bcl2l12 BCL2-like 12 (proline rich)

A_55_P2157902 Igsf10 immunoglobulin superfamily, mem

A_51_P216605 Hbp1 high mobility group box transcripti

A_55_P1967500 Nell1 NEL-like 1

A_51_P245546 Synrg

A_55_P2153496 Ppp2r3d protein phosphatase 2 (formerly 2.

A_55_P2049448 Phtf1 putative homeodomain transcriptic

A_51_P475628 Paqr6 progestin and adipoQ receptor fan

A_55_P1963687 Tsen54 tRNA splicing endonuclease $54 \mathrm{hc}$

A_66_P128927 Gm6306 predicted gene 6306

A_66_P133993 Gm5093 predicted gene 5093

A_51_P302942 Rasl10a RAS-like, family 10, member A

A_51_P341789 Sugp1 SURP and $G$ patch domain contai

A_66_P137383 Ddx55 DEAD (Asp-Glu-Ala-Asp) box poly

A_52_P640386 Usp53 ubiquitin specific peptidase 53

A_55_P2032318 4930522L14R RIKEN cDNA 4930522L14 gene

A_51_P487547 Ccdc91 coiled-coil domain containing 91

A_55_P1991500 Obfc1 oligonucleotide/oligosaccharide-bi

A_55_P2018847 Crlf2 cytokine receptor-like factor 2 
centromere protein $\mathrm{K}$ zinc finger protein 608 coiled-coil domain containing 114 solute carrier family 10 (sodium/bil Holliday junction recognition prote D-tyrosyl-tRNA deacylase 2 progestin and adipoQ receptor fan palmdelphin ferritin light chain 1

A_55_P2123831 Thap6 THAP domain containing 6

A_55_P1961241 LOC1026345؟ uncharacterized LOC102634598

A_55_P2063316 Mgll monoglyceride lipase

A_55_P1975832 1810009N02FRIKEN cDNA 1810009N02 gene

A_51_P331827 Slc25a41 solute carrier family 25, member 4

A_55_P2007919 Akr1c19 aldo-keto reductase family 1, mer

A_51_P240801 Tmem173 transmembrane protein 173

A_55_P2143075 Ubc ubiquitin C

A 55 P2145139 0

A_51_P469968 H2-M3 histocompatibility 2, M region locu A_55_P2057127 Nim1k NIM1 serine/threonine protein kine A_51_P298802 Bfsp2 beaded filament structural protein A_51_P468762 Alkbh6 alkB, alkylation repair homolog 6 ( A_55_P2097340 Camkv CaM kinase-like vesicle-associate A_55_P2053497 Poli polymerase (DNA directed), iota A 51_P437478 Zfp566 Zinc finger protein 566

A 52 P343627 Rbp7 retinol binding protein 7, cellular A_66_P126415 Gm8013 predicted gene 8013

A_55_P2106235 Syngr2 synaptogyrin 2

A_51_P116487 Lsm4

A_52_P472302 Fxyd6

A_55_P2008936 Slc2a9

A_52_P71105 Sertad3

A_52_P593037 Acsl5

A_55_P1998872 Slitrk5

A 66 P122699 Cux2

A_66_P128445 Bend7

A_51_P192139 Zfp74

A_66_P104309 Myl2

A_55_P2000304 Terf1

A 55 P2137701 Gm13247

A_55_P2007273 Pole

A_55_P1968200 Hjurp

A_52_P357055 Ccdc91

A_52_P211418 G2e3

A_51_P230439 Ppfibp2

A_55_P1961140 Mettl3

A_55_P2018666 Thrsp LSM4 homolog, U6 small nuclear FXYD domain-containing ion trans solute carrier family 2 (facilitated $g$ SERTA domain containing 3 acyl-CoA synthetase long-chain fa SLIT and NTRK-like family, memb cut-like homeobox 2

BEN domain containing 7

zinc finger protein 74

myosin, light polypeptide 2 , regula telomeric repeat binding factor 1 predicted gene 13247

polymerase (DNA directed), epsilc Holliday junction recognition prote coiled-coil domain containing 91 G2/M-phase specific E3 ubiquitin I PTPRF interacting protein, bindins methyltransferase like 3 thyroid hormone responsive A_55_P2223282 B130019D13FRIKEN cDNA B130019D13 gene A_55_P2227580 A130072N09FRIKEN cDNA A130072N09 gene A_55_P1971174 Cd1d2 A_55_P2084332 Pigp

A_51_P151586 Gsg2

A_55_P1968908 Hypk

A_55_P2019113 Apol7b

A_52_P367675 Acin1

A_51_P465273 Mettl17

A_51_P421223 Fv1

A_55_P2131340 Churc1

A_55_P2061371 Gm15698
CD1d2 antigen phosphatidylinositol glycan anchol germ cell-specific gene 2 huntingtin interacting protein $\mathrm{K}$ apolipoprotein $\mathrm{L} 7 \mathrm{~b}$ apoptotic chromatin condensation methyltransferase like 17 Friend virus susceptibility 1 churchill domain containing 1 transcription elongation factor $B(\varsigma$ 
1

2

3

4

5

6

7

8

9 $\begin{array}{llr}\text { A_55_P1970299 } & \text { Mttp } & \text { microsomal triglyceride transfer pr } \\ \text { A_51_P108581 } & \text { Adrbk2 } & \text { adrenergic receptor kinase, beta } 2 \\ \text { A_52_P67270 } & \text { 4930515G01F RIKEN cDNA 4930515G01 gene } \\ \text { A_55_P2121846 } & 0 \\ \text { A_55_P1968858 } & \text { Cadps } & \text { Ca2+-dependent secretion activat }\end{array}$

A_66_P111430 2410006H16F RIKEN cDNA 2410006H16 gene

A_52_P257686 Rwdd3 RWD domain containing 3

A_55_P2167323 Cort

A_51_P101573 Klc4

A 55 P2457154 G2e3

A 55 P2035951 Haus8

A_51_P455807 Ehd4

A_51_P253732 II17rd

A_55_P2143251 Rims3

A_51_P414126 Rab19

A_55_P2137941 Fxyd2

A_55_P2072656 Ckmt1

A 51 P312437 Dhrs7

A_52_P664404 Zfp286

A_55_P2000533 Polk

A_51_P353392 Cript

A_55_P2417936 AW125324

A_55_P2181009 Gpr180 cortistatin kinesin light chain 4 G2/M-phase specific E3 ubiquitin I 4HAUS augmin-like complex, subı EH-domain containing 4 interleukin 17 receptor $D$ regulating synaptic membrane exc RAB19, member RAS oncogene $f$ FXYD domain-containing ion trans creatine kinase, mitochondrial $1, u$ dehydrogenase/reductase (SDR f: zinc finger protein 286

polymerase (DNA directed), kappc cysteine-rich PDZ-binding protein expressed sequence AW125324

A 55 P2028936 5033425G24FRIKEN CDNA 5033425G24 gene

A 55 P2003541 Nrcam neuron-glia-CAM-related cell adh A 51 P310164 2810459M11F RIKEN cDNA 2810459M11 gene A_52_P338956 Aspg asparaginase homolog (S. cerevis A_66_P131169 LOC1008622€ uncharacterized LOC100862268 A_51_P223569 Ddx4 DEAD (Asp-Glu-Ala-Asp) box poly A_55_P1974487 Atp8b5 A_55_P2064257 Dnaic2 A_55_P2075213 Kbtbd11 A 55 P1953103 Nudt7 A_55_P2014100 Gm7120 A_55_P2108820 Adamts3 A_52_P1092823 Irx1 A_55_P2045085 Rpl34-ps1

A_52_P640922 Dcdc2a

A 51_P327874 Pth1r

A_52_P348031 Syt9

A_55_P2031692 Gstm6

A_55_P2111148 Gemin5

A_55_P2031496 Rufy3

A_52_P434306

A_55_P1984690 Ptprr

A_51_P431870 Map1s

A_55_P2060991 BC005764

A 51P218953 Zfp536

A_55_P1985410 Reps2 ATPase, class I, type 8B, member dynein, axonemal, intermediate cr kelch repeat and BTB (POZ) dom: nudix (nucleoside diphosphate link predicted gene 7120

a disintegrin-like and metallopeptic Iroquois related homeobox 1 (Dro: ribosomal protein L34, pseudogen doublecortin domain containing $2 \varepsilon$ parathyroid hormone 1 receptor synaptotagmin IX glutathione S-transferase, mu 6 gem (nuclear organelle) associate RUN and FYVE domain containin 0 protein tyrosine phosphatase, rect microtubule-associated protein $1 \mathrm{~S}$ cDNA sequence BC005764 zinc finger protein 536 RALBP1 associated Eps domain c family with sequence similarity 83 , guanine nucleotide binding proteir A 106011 teashirt zinc finger family member

A_51_P394833 Tshz1

A_51_P477019 Rnaset2a ribonuclease T2A

A_55_P2169311 4930515G01FRIKEN cDNA 4930515G01 gene

A_51_P461108 Osbpl10 oxysterol binding protein-like 10

A_55_P2149951 Prx periaxin

A_51_P116906 Rapgef3 Rap guanine nucleotide exchange A_55_P2108784 Arhgap22 Rho GTPase activating protein 22 A_51_P413507 2010109A12R RIKEN cDNA 2010109A12 gene 
A_51_P481159 Cbr3

carbonyl reductase 3

A_55_P1954092

0

A_66_P112024

A_55_P1979027 Tmem65

0

A_51_P451588 Plekhb1

transmembrane protein 65

A_55_P2045007 Hrh1

pleckstrin homology domain conta

histamine receptor $\mathrm{H} 1$

A_55_P2096368 LOC1026327iADP-ribosylation factor 1-like

A_55_P2115127 Mphosph10 M-phase phosphoprotein 10 (U3 s

A_55_P1975120 Gm10777

A_55_P2106150 Cenpk

predicted gene 10777

centromere protein $\mathrm{K}$

A_55_P2036813

A_55_P2007771 Catsper2

A_55_P2157966 Map1a

0

cation channel, sperm associated microtubule-associated protein $1 \mathrm{f}$ TRAF-interacting protein protein tyrosine phosphatase, non seven in absentia homolog 3 (Dro: heparanase iduronidase, alpha-Lpredicted gene 2696 mannan-binding lectin serine pept ferritin light chain 1 bisphosphate 3'-nucleotidase 1 synaptoporin synuclein, gamma

A 55-P2185950 Bpnt1

A 55 P2187076 Sncg

A_55_P2121156 LOC10263421 uncharacterized LOC102634215

A_55_P2099540 H2afj

A_55_P2169227 Ccdc184

A_55_P2105362 Tmed5

A_51_P215374 Slc6a17

A_55_P2059986 Chst14

A_52_P558609 Clec16a

A_55_P2179834 Gatad2a

A_51_P273609 Itpka

A_55_P2124026 Synj2

$\mathrm{H} 2 \mathrm{~A}$ histone family, member $\mathrm{J}$ coiled-coil domain containing 184 transmembrane emp24 protein tra solute carrier family 6 (neurotransı carbohydrate ( $\mathrm{N}$-acetylgalactosar C-type lectin domain family 16, me GATA zinc finger domain containir inositol 1,4,5-trisphosphate 3-kina: synaptojanin 2

A_51_P348617 2310045N01FRIKEN cDNA 2310045N01 gene A_51_P408989 L3hypdh L-3-hydroxyproline dehydratase $(\mathrm{t}$ A_51_P331661 Ubiad1 UbiA prenyltransferase domain co A_55_P1998299 Itgb4

A_55_P2119892 Erbb4

A_55_P1958597 Slc27a3

A_55_P2105220 Hscb

A_55_P2059179 Slc48a1

A_66_P138584 Mnd1

A_52_P116264 Adhfe1

A_52_P650325 Slc35e1

A_55_P2095909 Lamtor3

A_52_P489295 Adamts1

A_65_P01247 Hjurp

A_55_P1953387 Fabp5

A_55_P2023707 Camta1 integrin beta 4 v-erb-a erythroblastic leukemia vir solute carrier family 27 (fatty acid . HscB iron-sulfur cluster co-chaper solute carrier family 48 (heme tran meiotic nuclear divisions 1 homolc alcohol dehydrogenase, iron conte solute carrier family 35 , member $\mathrm{E}$ late endosomal/lysosomal adaptor a disintegrin-like and metallopeptic Holliday junction recognition prote fatty acid binding protein 5 , epider E330013P04FRIKEN cDNA E330013P04 gene A_51_P134812 Chac1 ChaC, cation transport regulator 1 A_55_P1961014 Selenbp1 A_52_P529195 Pcdhb4 selenium binding protein 1

A_55_P1963508 Slc13a5 solute carrier family 13 (sodium-de

A_55_P2004960 9130017K11RRIKEN cDNA 9130017K11 gene

A_55_P2072391 Acp1 acid phosphatase 1, soluble

A_55_P2183914 Gm7120 predicted gene 7120

A_51_P147034 Ica1l islet cell autoantigen 1-like g

.


1

2

3

4

5

6

7

8

9

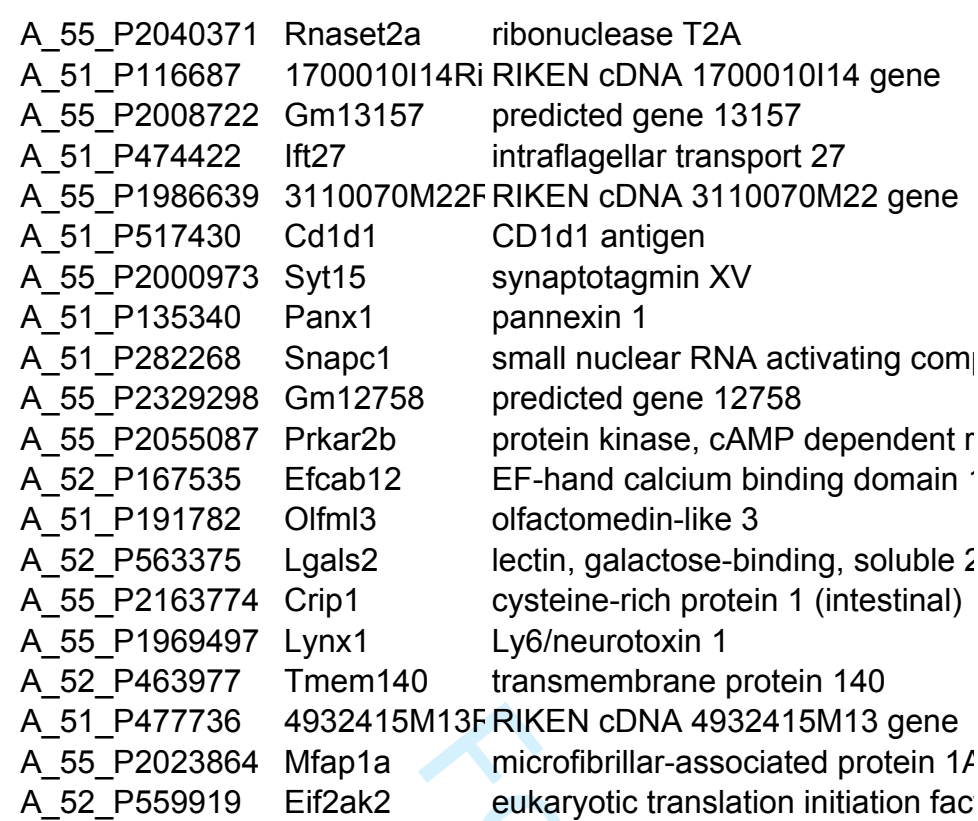

A_55_P2356736 6720483E21RRIKEN cDNA 6720483E21 gene

A_55_P2052834 Lst1 leukocyte specific transcript 1

A_55_P1953919 Me3 malic enzyme 3, NADP(+)-depenc

A_51_P446796 Camta1 calmodulin binding transcription ac

A_51_P464308 Gnb4 guanine nucleotide binding proteir

A 55 P2001628 Rps4l ribosomal protein S4-like

A_52_P38908 Tmem132b transmembrane protein 132B

A_51_P283968 Adamts18 a disintegrin-like and metallopeptic

A_52_P630867 Abcc4 ATP-binding cassette, sub-family 1

A_55_P1953920 Me3 malic enzyme 3, NADP(+)-depenc

A_55_P2105321 Acin1 apoptotic chromatin condensation

A_51_P502437 Cacna2d3 calcium channel, voltage-depende

A_51_P369803 Psmb9 proteasome (prosome, macropain

A_55_P1953087 Mcm3 minichromosome maintenance de

A_55_P2115567 Slc26a1 solute carrier family 26 (sulfate tra

A_55_P2044242 Slc13a5 solute carrier family 13 (sodium-dt

A_55_P2079619 Rnf43 ring finger protein 43

A_55_P1959076 Zfp930 zinc finger protein 930

A_55_P2048119 Slc29a4 solute carrier family 29 (nucleosidı

A_52_P400509 Atm ataxia telangiectasia mutated hom

A_51_P506733 P2rx7 purinergic receptor P2X, ligand-ga

A_55_P1973352 0

A_55_P2008417 Mnd1 meiotic nuclear divisions 1 homolc

A_55_P2106459 Zfhx3 zinc finger homeobox 3

A_55_P2370160 C130009A20F RIKEN cDNA C130009A20 gene

A_51_P417251 6330403K07RRIKEN cDNA 6330403K07 gene

A_55_P2067116 Dclre1c DNA cross-link repair 1C, PSO2 h

A_65_P03606 Rpap2 RNA polymerase II associated prc

A_55_P2062593 Gm4924 predicted gene 4924

A_55_P2155644 2010315B03RRIKEN cDNA 2010315B03 gene

A_55_P2075313 Zfp619 zinc finger protein 619

A_55_P2048478 Olfml1 olfactomedin-like 1

A_55_P2018181 A730008H23F RIKEN cDNA A730008H23 gene

A_52_P481279 Drc1 dynein regulatory complex subunit

A_55_P2073642 1600014C10FRIKEN cDNA 1600014C10 gene

A_55_P2123683 Chrdl1 chordin-like 1

A_55_P2016034 Nirc5 NLR family, CARD domain contair A_55_P2035424 Hpgd hydroxyprostaglandin dehydrogen

A_55_P2059090 Tagap T cell activation Rho GTPase acti 

A_52_P305230 Igsf21
A_55_P1960735 Gdf15
A_55_P2090330 Kcnmb4
A 52_P574653 Bid
A_55_P2080880 Clcnkb
A_52_P590535 Fbln2
A_65_P01834 Lima1
A_55_P2116650
A_55_P2056557
A_55_P2079116
immunoglobulin superfamily, mem growth differentiation factor 15 potassium large conductance calc $\mathrm{BH} 3$ interacting domain death ago chloride channel $\mathrm{Kb}$
fibulin 2
LIM domain and actin binding 1 0
0
0

A 55 P2057622 Ocel1

A_55_P2041828 Tubb3

A_52_P124472 Kcnd2

A_55_P2334927

\section{$9.13 \mathrm{E}+1$}

A_52_P573255 Cdc42ep1

A_55_P2147280 Myh1

A_55_P1983958 Gm20746

A_66_P122415 Snhg6

A_52_P48546 Rnf41

A_52_P1042732 Akap5

A_55_P2076303 March8

A_55_P2154977 Rpap3

A_55_P2006035 Galnt15

A_55_P2133205 Nmt2

A_55_P1957213 3930401B19R

A_55_P2115151 Pxylp1

A_51_P506937 Mrps12

A_55_P2110290 Tmem254a

A_55_P2088720

A_55_P2209258

A_52_P250555

A_55_P2027852

A_52_P8324

A_52_P441294

A_55_P1967553

A_51_P241210 Lhfpl3

A_55_P2029746

A_55_P1954724

A_51_P337944 Bmp2k

A_55_P2007673 Lrsam1

A_52_P447196 Col4a6

A_52_P650379 Strap

A_55_P1976898 Pnp2

A_55_P1994339 Dhrs7c

occludin/ELL domain containing 1 tubulin, beta 3 class III potassium voltage-gated channel, uncharacterized 9130022E09 CDC42 effector protein (Rho GTP myosin, heavy polypeptide 1 , skel predicted gene, 20746 small nucleolar RNA host gene 6 ring finger protein 41

A kinase (PRKA) anchor protein 5 membrane-associated ring finger I RNA polymerase II associated prc UDP-N-acetyl-alpha-D-galactosan $\mathrm{N}$-myristoyltransferase 2

\section{REN CDNA 3930401B19 gene} 2-phosphoxylose phosphatase 1 mitochondrial ribosomal protein $\mathrm{S}$. transmembrane protein $254 a$

0

pituitary tumor-transforming gene

A_55_P2011659 Anapc5 anaphase-promoting complex sub

A_55_P2213214 A030001D20F RIKEN cDNA A030001D20 gene

A_51_P140690 Stmn3 stathmin-like 3

A_55_P2013223 S100z S100 calcium binding protein, zete

A_52_P331762 Lmo1 LIM domain only 1

A_51_P355427 Timp4 tissue inhibitor of metalloproteinas

A_51_P382970 Itga9 integrin alpha 9

A_51_P497985 C2 complement component 2 (within

A_51_P360918 Ehd3 EH-domain containing 3

A_51_P309854 Kcnn2 potassium intermediate/small conc

A_66_P125035 Duxbl2 doubl homeobox B-like 2

A_55_P2063256 Lgals4

A_51_P497100 Lgals4 lectin, galactose binding, soluble 4 lectin, galactose binding, soluble 4 
1

2

3

4

5

6

7

8

9

\begin{tabular}{|c|c|c|}
\hline _51_P129149 & Gatad2a & I A Zinc finger domain containi \\
\hline 55_P2148534 & $\mathrm{Nr} 1 \mathrm{~d} 2$ & lear receptor subfamily 1 , grou \\
\hline A_55_P2010936 & Fbxo17 & F-box protein 17 \\
\hline A_55_P2033425 & Grm5 & glutamate receptor, metabotropic \\
\hline 51_P292357 & Rps3a1 & ribosomal protein S3A1 \\
\hline 52_P257625 & Esm1 & endothelial cell-specific molecule \\
\hline A_55_P2058791 & $4833420 \mathrm{G} 17 \mathrm{~F}$ & FRIKEN cDNA 4833420G17 gene \\
\hline A_55_P2106525 & Nmnat3 & nicotinamide nucleotide adenyly \\
\hline A_55_P2040600 & Exd1 & exonuclease $3^{\prime}-5^{\prime}$ domain contai \\
\hline A_55_P2140212 & 0 & 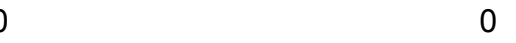 \\
\hline A_55_P2093774 & Chl1 & cell adhesion molecule with hom \\
\hline A_52_P165773 & Prmt8 & protein arginine $\mathrm{N}$-methyltra \\
\hline A_55_P2014304 & Kank1 & $\mathrm{KN}$ \\
\hline A_51_P458778 & Hpgd & hydroxyprostaglandin dehydroge \\
\hline A_55_P2162344 & Lrsam1 & leucine rich repeat and sterile alpr \\
\hline A_55_P2004179 & Col2a1 & collagen, type II, a \\
\hline A_55_P2096867 & Gap43 & grol \\
\hline A_55_P1968276 & Tomm22 & ondrial \\
\hline A_55_P2315012 & 4930458D05F & \\
\hline A_55_P1978465 & H2-Q5 & \\
\hline A_55_P1953972 & Pdh & \\
\hline A_55_P2212603 & Apba2 & \\
\hline A_51_P194249 & Stmn4 & stath \\
\hline A_51_P128667 & Lynx1 & Ly6/n \\
\hline A_66_P105175 & Bche & butyryl \\
\hline A_55_P2176792 & Sh3gl3 & 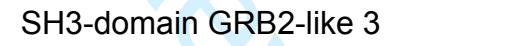 \\
\hline A_66_P10 & Ucp3 & unce \\
\hline A_55_P2045642 & Stmn4 & \\
\hline A_55_P209 & Padi2 & \\
\hline A_52_P597 & Gprc5a & ed receptor, family \\
\hline A_51_P & Lpl & \\
\hline A_52_P2578 & Lpl & lipopr \\
\hline A_55_P2074656 & Padi2 & hine deiminase, type \\
\hline A_51_P297105 & Ucp2 & nitochondria \\
\hline A_52_P157450 & Abhd1 & ontaining 1 \\
\hline 613498 & 4833420G17F & F RIKEN cDNA 4833420G17 gene \\
\hline A_51_P159453 & Serpina3n & steine) peptidase inhi \\
\hline A_55_P2026270 & $\mathrm{Cfi}$ & nt factor $\mathrm{i}$ \\
\hline A_51_ & Galnt15 & UDP-N-acetyl-alpha-D-galactos \\
\hline & & \\
\hline A_55_P1C & Pbp2 & indin \\
\hline A_55 & Olfr224 & \\
\hline A_52_P206492 & Pop4 & proce \\
\hline A_55_P2026420 & Pou6f1 & class 6 , transcriptiol \\
\hline A_52_P111 & Pcdh17 & \\
\hline A_55_P2137527 & Fam183b & family with sequence similarity 18 \\
\hline A_55_P2132207 & 1810037I17Ri & i RIKEN cDNA 1810037117 gene \\
\hline A_52_P303176 & 1810037I17Ri & Ri RIKEN cDNA $1810037 / 17$ gene \\
\hline A_66_P130813 & Samd4 & sterile alpha motif domain conta \\
\hline A_55_P1966774 & Serpina3i & serine (or cysteine) peptidase in \\
\hline & & \\
\hline A 55 P1966838 & Xaf1 & XIAP associated factor 1 \\
\hline
\end{tabular}

A_55_P2148534 Nr1d2

A 55_P2010936 Fbxo17

A_55_P2033425 Grm5

A 51 P292357 Rps3a1

A_55_P2058791 4833420G17FRIKEN cDNA 4833420G17 gene

A_55_P2106525 Nmnat3 nicotinamide nucleotide adenylyltr

A_55_P2040600 Exd1

P2140212

A_55_P2093774 Chl1

A_52_P165773 Prmt8

A_55_P2014304 Kank1

A 55 P200417

A_55_P2096867 Gap43

A 55 P1968276 Tomm22

A_55_P2315012 4930458D05F R

A 55 P1978465 H2-Q5

A 55 P1953972 Pdhb

A 55 P2212603 Apba2

A 66 P105175

A 55 P2176792 Sh3gl3

A 66 P108247 Ucp3

A_51_P259296 Lpl

A 52_P257812 Lp

Serpina3n

A 55 P2026270 Cf

A 52 P393314 P2rx7

A_55_P1952482 Pbp2

A 55 P2105944 Olfr224 


\section{g and 3xTg-AD primary hippocampal astrocytes Description}

Mus musculus tripartite motif-containing 12A (Trim12a), mRNA [NM 023835]

Mus musculus 13 days embryo male testis cDNA, RIKEN full-length enriched library, clone:6030400B02 pr Mus musculus chemokine ( $\mathrm{C}-\mathrm{C}$ motif) ligand 27A (Ccl27a), transcript variant 1, mRNA [NM_001048179]

Mus musculus RIKEN cDNA 2610507101 gene (2610507I01Rik), long non-coding RNA [NR_037964]

Mus musculus tripartite motif-containing 12A (Trim12a), mRNA [NM_023835]

Mus musculus 16 days embryo head cDNA, RIKEN full-length enriched library, clone:C130078N14 product Mus musculus predicted gene, 20878 (Gm20878), mRNA [NM_001270431]

Mus musculus microtubule-associated protein 1B (Map1b), mRNA [NM_008634]

Mus musculus predicted gene, 20878 (Gm20878), mRNA [NM_001270431]

Mus musculus carbonic anhydrase 9 (Car9), mRNA [NM_139305]

Mus musculus vomeronasal 2, receptor 123 (Vmn2r123), mRNA [NM_009485]

Mus musculus angiogenin, ribonuclease A family, member 2 (Ang2), mRNA [NM_007449]

Mus musculus zinc finger protein 933 (Zfp933), mRNA [NM_198619]

Mus musculus bisphosphate 3'-nucleotidase 1 (Bpnt1), mRNA [NM_011794]

Mus musculus jumonji domain containing 7 (Jmjd7), mRNA [NM_001114637]

Mus musculus zinc finger protein 963 (Zfp963), mRNA [NM_001200023]

Mus musculus kallikrein 1-related peptidase b22 (KIk1b22), mRNA [NM_010114]

Mus musculus cDNA sequence BC049715 (BC049715), mRNA [NM_178776]

Mus musculus patched domain containing 2 (Ptchd2), mRNA [NM_001083342]

Mus musculus carbohydrate ( $\mathrm{N}$-acetylgalactosamine 4-0) sulfotransferase 8 (Chst8), mRNA [NM_175140] Mus musculus cDNA sequence BC049715 (BC049715), mRNA [NM_178776]

Mus musculus solute carrier family 2 (facilitated glucose transporter), member 4 (SIc2a4), mRNA [NM_009: Mus musculus serine/threonine kinase 3 (Stk3), mRNA [NM_019635]

Mus musculus occludin/ELL domain containing 1 (Ocel1), mRNA [NM_029865]

Mus musculus RIKEN cDNA 4930427A07 gene (4930427A07Rik), mRNA [NM_134041]

Mus musculus tripartite motif-containing 34A (Trim34a), mRNA [NM_030684]

Mus musculus angiogenin, ribonuclease A family, member 3 (Ang3), mRNA [NM_001123394]

PREDICTED: Mus musculus tropomyosin alpha-4 chain-like (LOC102633627), misc_RNA [XR_385459]

Mus musculus major facilitator superfamily domain containing 7B (Mfsd7b), mRNA [NM_001081259]

Mus musculus secreted phosphoprotein 1 (Spp1), transcript variant 5, mRNA [NM_001204233]

Mus musculus bone morphogenetic protein 4 (Bmp4), mRNA [NM_007554]

Mus musculus kallikrein 1-related peptidase b3 (Klk1b3), mRNA [NM_008693]

Mus musculus ST6 (alpha-N-acetyl-neuraminyl-2,3-beta-galactosyl-1,3)-N-acetylgalactosaminide alpha-2,6 Mus musculus 2-deoxyribose-5-phosphate aldolase homolog (C. elegans) (Dera), mRNA [NM_172733] Mus musculus coiled-coil domain containing 65 (Ccdc65), mRNA [NM_153518]

Mus musculus phorbol-12-myristate-13-acetate-induced protein 1 (Pmaip1), mRNA [NM_021451]

Mus musculus potassium voltage-gated channel, shaker-related subfamily, beta member 1 (Kcnab1), trans Mus musculus adult male tongue cDNA, RIKEN full-length enriched library, clone:2310058N22 product:unc Mus musculus RIKEN cDNA 9030025P20 gene (9030025P20Rik), mRNA [NM_001123370]

Mus musculus mutL homolog 1 (E. coli) (Mlh1), mRNA [NM_026810]

Mus musculus CD59a antigen (Cd59a), transcript variant 2, mRNA [NM_007652]

Mus musculus leucine zipper protein 2 (Luzp2), mRNA [NM_178705]

Mus musculus zinc finger protein 236 (Zfp236), mRNA [NM_177832]

Mus musculus cortexin 3 (Ctxn3), mRNA [NM_001134697]

Mus musculus chemokine (C-C motif) ligand 27A (Ccl27a), transcript variant 2 (and 1), mRNA [NM_011336 Mus musculus MAM domain containing 2 (Mamdc2), mRNA [NM_174857]

Mus musculus chemokine (C-C motif) ligand 27A (Ccl27a), transcript variant 1, mRNA [NM_001048179]

Mus musculus zinc finger protein 961 (Zfp961), mRNA [NM_001164581]

Mus musculus podoplanin (Pdpn), transcript variant 1, mRNA [NM_010329]

Mus musculus LanC lantibiotic synthetase component C-like 3 (bacterial) (Lancl3), mRNA [NM_173414]

Mus musculus microtubule associated serine/threonine kinase family member 4 (Mast4), mRNA [NM_1751 Mus musculus RIKEN cDNA 1500011B03 gene (1500011B03Rik), transcript variant 2, long non-coding RN Mus musculus ER membrane associated RNA degradation (Ermard), transcript variant 1, mRNA [NM_001( Mus musculus transmembrane protein 88 (Tmem88), mRNA [NM_025915]

Mus musculus ER membrane associated RNA degradation (Ermard), transcript variant 1, mRNA [NM_001( Mus musculus myosin VIIA (Myo7a), transcript variant 2, mRNA [NM_008663] 
Mus musculus tetratricopeptide repeat domain 8 (Ttc8), transcript variant 1, mRNA [NM_029553]

Mus musculus lung RCB-0558 LLC cDNA, RIKEN full-length enriched library, clone:G730014H17 product:L Mus musculus matrix-remodelling associated 7 (Mxra7), mRNA [NM_026280]

PREDICTED: Mus musculus tumor necrosis factor (ligand) superfamily, member 13, opposite strand (Tnfsf Mus musculus centrosomal protein 76 (Cep76), mRNA [NM_001081073] sorting nexin 29 [Source:MGI Symbol;Acc:MGI:1921728] [ENNSMUST00000134941] GAG_IPMAE (P31790) Retrovirus-related Gag polyprotein, partial (43\%) [TC1705975] Mus musculus cDNA sequence BC006965 (BC006965), long non-coding RNA [NR_024085] Mus musculus ATPase, class V, type 10D (Atp10d), mRNA [NM_153389] Mus musculus angiogenin, ribonuclease A family, member 3 (Ang3), mRNA [NM_001123394] Mus musculus predicted gene 13152 (Gm13152), mRNA [NM_001039209] Mus musculus cerebral endothelial cell adhesion molecule (Cercam), mRNA [NM_207298] coiled-coil domain containing 32 [Source:MGI Symbol;Acc:MGI:2685477] [ENSMUSST00000110834] Mus musculus cAMP responsive element binding protein 3 (Creb3), mRNA [NM_013497] Mus musculus RIKEN cDNA 1190007 I07 gene (1190007I07Rik), transcript variant 1, mRNA [NM_0011355 Mus musculus purinergic receptor P2Y, G-protein coupled 2 (P2ry2), mRNA [NM_008773] Mus musculus 6 days neonate head cDNA, RIKEN full-length enriched library, clone:5430416G10 product: Mus musculus phosphatidylserine decarboxylase, pseudogene 3 (Pisd-ps3), non-coding RNA [NR_003518 Mus musculus diacylglycerol O-acyltransferase 2 (Dgat2), mRNA [NM_026384]

Mus musculus family with sequence similarity 32, member A (Fam32a), mRNA [NM_026455]

Mus musculus histidyl-tRNA synthetase (Hars), mRNA [NM_008214]

Mus musculus acetyl-Coenzyme A carboxylase beta (Acacb), mRNA [NM_133904]

Mus musculus predicted gene 3448 (Gm3448), mRNA [NM_001123367]

Mus musculus branched chain aminotransferase 2, mitochondrial (Bcat2), transcript variant 2, mRNA [NM Mus musculus carbonic anhydrase 11 (Car11), mRNA [NM_009800]

Mus musculus immunity-related GTPase family M member 2 (Irgm2), mRNA [NM 019440]

Mus musculus predicted gene, EG633640, mRNA (cDNA clone MGC:118117 IMAGE:6309338), complete

Mus musculus La ribonucleoprotein domain family, member 1 (Larp1), mRNA [NM_028451]

Mus musculus BCLI2-like 15 (Bcl2I15), transcript variant 1, mRNA [NM_001142959]

Mus musculus nuclear receptor co-repressor 2 (Ncor2), transcript variant 1, mRNA [NM_011424]

Mus musculus adult male spinal cord cDNA, RIKEN full-length enriched library, clone:A330057J19 product: docking protein 7 [Source:MGI Symbol;Acc:MGI:3584043] [ENSMUST00000114270]

Mus musculus F-box protein 44 (Fbxo44), transcript variant 1, mRNA [NM_173401]

Mus musculus purine rich element binding protein B (Purb), mRNA [NM_011221]

Mus musculus expressed sequence AI593442 (Al593442), transcript variant 1, mRNA [NM_001286641]

Mus musculus DNA-damage-inducible transcript 4-like (Ddit4I), mRNA [NM_030143]

Mus musculus wingless-related MMTV integration site 2 (Wnt2), mRNA [NM_023653]

Mus musculus annexin A3 (Anxa3), mRNA [NM_013470]

eukaryotic translation initiation factor 2 alpha kinase 4 [Source:MGI Symbol;Acc:MGI:1353427] [ENSMUST Mus musculus family with sequence similarity 154, member B (Fam154b), mRNA [NM_177894]

Mus musculus zinc finger protein 386 (Kruppel-like) (Zfp386), transcript variant 1, mRNA [NM_001004066] Mus musculus ribonuclease, RNase A family 4 (Rnase4), transcript variant 1, mRNA [NM_021472]

Mus musculus mitogen-activated protein kinase kinase 7 (Map2k7), transcript variant 1, mRNA [NM_00104 Mus musculus zinc finger protein 672 (Zfp672), transcript variant 2, non-coding RNA [NR_028331]

Mus musculus patched domain containing 2 (Ptchd2), mRNA [NM_001083342]

Mus musculus small integral membrane protein 5 (Smim5), mRNA [NM_183259]

Mus musculus dynein light chain Tctex-type 1F (Dynlt1f), transcript variant 2, mRNA [NM_001199948]

Mus musculus solute carrier family 30 (zinc transporter), member 7 (SIc30a7), mRNA [NM_023214]

Mus musculus tRNA nucleotidyl transferase, CCA-adding, 1 (Trnt1), transcript variant 1, mRNA [NM_0012 Mus musculus tyrosine 3-monooxygenase/tryptophan 5-monooxygenase activation protein, zeta polypeptid Mus musculus RIKEN cDNA 4930444P10 gene (4930444P10Rik), mRNA [NM_001243238]

Mus musculus START domain containing 10 (Stard10), mRNA [NM_019990]

Mus musculus serine/threonine kinase 38 like (Stk38I), mRNA [NM_172734]

Mus musculus RIKEN cDNA 1700001C19 gene (1700001C19Rik), transcript variant 1, mRNA [NM_029296 Mus musculus predicted gene 13298 (Gm13298), mRNA [NM_001085530]

Mus musculus peptidase D (Pepd), mRNA [NM_008820]

Mus musculus polyadenylate binding protein-interacting protein 1 (Paip1), transcript variant 1, mRNA [NM_ Mus musculus Smith-Magenis syndrome chromosome region, candidate 8 homolog (human) (Smcr8), tran: PREDICTED: Mus musculus pregnancy specific glycoprotein 16 (Psg16), transcript variant X2, mRNA [XM. 
Mus musculus growth factor receptor bound protein 14 (Grb14), mRNA [NM_016719]

Mus musculus predicted gene 10845 (Gm10845), long non-coding RNA [NR_033535]

Mus musculus ribosomal protein L15 (Rpl15), mRNA [NM_025586]

Mus musculus cDNA clone IMAGE:5065404. [BC029971]

Mus musculus dedicator of cytokinesis 4 (Dock4), mRNA [NM_172803]

Mus musculus retinal degeneration $3(\mathrm{Rd} 3)$, transcript variant 1 , mRNA [NM_023727]

Mus musculus vimentin-type intermediate filament associated coiled-coil protein (Vmac), transcript variant Mus musculus predicted gene, 547127, mRNA (cDNA clone IMAGE:577088), complete cds. [BC096660] Mus musculus predicted gene 3448 (Gm3448), mRNA [NM_001123367]

Mus musculus adult male olfactory brain cDNA, RIKEN full-length enriched library, clone:6430517C21 prod Mus musculus four and a half LIM domains 2 (Fhl2), transcript variant 1, mRNA [NM 010212]

Mus musculus fibronectin type III domain containing 1 (Fndc1), mRNA [NM_001081416]

Mus musculus zinc finger, B-box domain containing (Zbbx), mRNA [NM_172515]

Mus musculus predicted gene 13157 (Gm13157), mRNA [NM_001127189]

Mus musculus ribosomal protein L31 (Rpl31), transcript variant 3, mRNA [NM_053257]

Mus musculus ring finger protein 41 (Rnf41), transcript variant 1, mRNA [NM_001164237]

Mus musculus adult male aorta and vein cDNA, RIKEN full-length enriched library, clone:A530041D01 proc Mus musculus galactosidase, beta 1 (Glb1), transcript variant 1, mRNA [NM_009752]

Mus musculus zinc finger protein 420 (Zfp420), mRNA [NM 172740]

Mus musculus ribosomal protein S13 (Rps13), mRNA [NM_026533]

Mus musculus maestro (Mro), mRNA [NM_027741]

Mus musculus myocilin (Myoc), mRNA [NM_010865]

Mus musculus peptidylprolyl isomerase domain and WD repeat containing 1 (Ppwd1), mRNA [NM_172807. Mus musculus tubulin tyrosine ligase-like family, member 2 (TtIl2), mRNA [NM_001098267] Mus musculus WD repeat domain 60 (Wdr60), mRNA [NM_146039]

Mus musculus OCIA domain containing 1 (Ociad1), transcript variant 1, mRNA [NM 023429]

Mus musculus SPEG complex locus (Speg), transcript variant 3, mRNA [NM_001085371]

Mus musculus actin-binding LIM protein 1 (Ablim1), transcript variant 1, mRNA [NM 178688]

Mus musculus autophagy related 16-like 2 (S. cerevisiae) (Atg16I2), mRNA [NM_001111111]

Mus musculus 4 days neonate thymus cDNA, RIKEN full-length enriched library, clone:B630008G01 produ Mus musculus autophagy related 16-like 2 (S. cerevisiae) (Atg16I2), mRNA [NM_001111111]

Mus musculus tripartite motif-containing 16 (Trim16), mRNA [NM_053169]

Mus musculus coiled-coil domain containing 65 (Ccdc65), mRNA [NM_153518]

Mus musculus solute carrier family 9 (sodium/hydrogen exchanger), member 3 regulator 1 (Slc9a3r1), mR Mus musculus period circadian clock 1 (Per1), transcript variant 1, mRNA [NM_011065] Mus musculus protein kinase, cAMP dependent regulatory, type II alpha (Prkar2a), mRNA [NM_008924] Mus musculus predicted gene 10516 (Gm10516), long non-coding RNA [NR_033536]

Mus musculus vomeronasal 2, receptor 121 (Vmn2r121), mRNA [NM_001100616] Mus musculus ets variant gene 3 (Etv3), transcript variant 1, mRNA [NM_001286844] Mus musculus nuclear receptor 2C2-associated protein (Nr2c2ap), transcript variant 1, mRNA [NM_00102! Mus musculus hormonally upregulated Neu-associated kinase (Hunk), mRNA [NM_015755] Mus musculus asunder, spermatogenesis regulator (Asun), mRNA [NM_138757] Mus musculus potassium voltage-gated channel, subfamily $\mathrm{H}$ (eag-related), member 5 (Kcnh5), mRNA [NN Mus musculus chemokine (C-X-C motif) ligand 14 (Cxcl14), mRNA [NM_019568]

Mus musculus insulin-like growth factor binding protein 3 (Igfbp3), mRNA [NM_008343]

Mus musculus serine/threonine kinase 38 like (Stk38I), mRNA [NM_172734]

Mus musculus epidermal growth factor receptor pathway substrate 8 (Eps8), transcript variant 1, mRNA [NI PREDICTED: Mus musculus uncharacterized LOC102639358 (LOC102639358), transcript variant X1, ncR Mus musculus signal peptidase complex subunit 3 homolog (S. cerevisiae) (Spcs3), mRNA [NM_029701] Mus musculus distal-less homeobox 2 (DIx2), mRNA [NM 010054]

Mus musculus docking protein 7 (Dok7), mRNA [NM_172708]

Mus musculus musashi RNA-binding protein 1 (Msi1), mRNA [NM_008629]

Mus musculus zinc finger protein 386 (Kruppel-like) (Zfp386), transcript variant 1, mRNA [NM_001004066] Mus musculus ATPase, class V, type 10A (Atp10a), mRNA [NM_009728]

Mus musculus matrix-remodelling associated 7 (Mxra7), mRNA [NM 026280]

Mus musculus dynein, axonemal, heavy chain 7A (Dnah7a), mRNA [NM_001252070]

Mus musculus frizzled homolog 6 (Drosophila) (Fzd6), transcript variant 1, mRNA [NM_008056]

Mus musculus serine/threonine kinase 36 (Stk36), mRNA [NM_175031]

Mus musculus Parkinson disease (autosomal recessive, juvenile) 2, parkin (Park2), mRNA [NM_016694] 
Mus musculus 2 cells egg cDNA, RIKEN full-length enriched library, clone:B020040A10 product:unclassifia PREDICTED: Mus musculus RIKEN cDNA C230072F16 gene (C230072F16Rik), transcript variant X3, ncF Mus musculus dual adaptor for phosphotyrosine and 3-phosphoinositides 1 (Dapp1), mRNA [NM_011932] Mus musculus LYR motif containing 5 (Lyrm5), transcript variant 1, mRNA [NM_001163628]

Mus musculus serine/threonine kinase 38 like (Stk38I), mRNA [NM_172734]

Mus musculus DNA segment, Chr 7, ERATO Doi 143, expressed (D7Ertd143e), long non-coding RNA [NR Mus musculus RIKEN cDNA 5830444B04 gene (5830444B04Rik), transcript variant 1, long non-coding RN Mus musculus dynein light chain Tctex-type 1C (Dynlt1c), mRNA [NM_001166630]

Mus musculus CDGSH iron sulfur domain 2 (Cisd2), mRNA [NM_025902]

Mus musculus camello-like 5 (Cml5), mRNA [NM 023493]

BB001409 RIKEN full-length enriched, 13 days embryo head Mus musculus cDNA clone 3110026C13 3'. [I Mus musculus ATP-binding cassette, sub-family D (ALD), member 1 (Abcd1), mRNA [NM_007435]

Mus musculus phosphatidylserine decarboxylase (Pisd), mRNA [NM_177298]

Mus musculus cat eye syndrome chromosome region, candidate 5 (Cecr5), mRNA [NM_144815]

Mus musculus NFKB inhibitor interacting Ras-like protein 1 (Nkiras1), mRNA [NM_023526]

Mus musculus FH2 domain containing 1 (Fhdc1), transcript variant 1, mRNA [NM_001205355]

Mus musculus zinc finger protein 14 (Zfp14), transcript variant 1, mRNA [NM_011748]

Mus musculus predicted gene 13298 (Gm13298), mRNA [NM_001085530]

Mus musculus Yip1 domain family, member 4 (Yipf4), mRNA [NM_026417]

Mus musculus kelch-like 26 (KIhl26), transcript variant 2, mRNA [NM_178771]

Mus musculus Williams-Beuren syndrome chromosome region 17 homolog (human) (Wbscr17), mRNA [Nז Mus musculus sperm associated antigen 1 (Spag1), mRNA [NM_012031]

Mus musculus cytochrome c oxidase assembly protein 18 (Cox18), transcript variant 1, mRNA [NM_00116 Mus musculus DAZ interacting protein 1 (Dzip1), mRNA [NM_025943]

Mus musculus trinucleotide repeat containing 18 (Tnrc18), transcript variant A, mRNA [NM_001122730] Mus musculus NLR family, pyrin domain containing 5 (NIrp5), transcript variant 1, mRNA [NM_011860] Mus musculus septin 9 (Sept9), transcript variant 2, mRNA [NM_001113487]

Mus musculus nicotinamide nucleotide transhydrogenase (Nnt), transcript variant 1, mRNA [NM_008710] Mus musculus 16 days neonate thymus cDNA, RIKEN full-length enriched library, clone:A130026C10 prod Mus musculus transmembrane protein 242 (Tmem242), mRNA [NM_027457] Mus musculus transmembrane protein 242 (Tmem242), mRNA [NM_027457] Mus musculus 16 days neonate thymus cDNA, RIKEN full-length enriched library, clone:A130009K03 prodı Mus musculus biregional cell adhesion molecule-related/down-regulated by oncogenes (Cdon) binding prol Mus musculus predicted gene, OTTMUSG00000016609 (OTTMUSG00000016609), mRNA [NM_0011004 Mus musculus dual specificity phosphatase 8 (Dusp8), mRNA [NM_008748] Mus musculus purine rich element binding protein B (Purb), mRNA [NM_011221] Mus musculus tumor necrosis factor receptor superfamily, member 11b (osteoprotegerin) (Tnfrsf11b), mRA Mus musculus 2 days neonate thymus thymic cells cDNA, RIKEN full-length enriched library, clone:E4300C Mus musculus phosphatidylserine decarboxylase, pseudogene 1 (Pisd-ps1), non-coding RNA [NR_003517 Mus musculus mitchondrial ribosomal protein S7 (Mrps7), mRNA [NM_025305]

Mus musculus polymerase (DNA directed), delta 2, regulatory subunit (Pold2), mRNA [NM_008894]

Mus musculus trans-golgi network vesicle protein 23A (Tvp23a), mRNA [NM_001013778]

Mus musculus F-box protein 44 (Fbxo44), transcript variant 1, mRNA [NM_173401]

Mus musculus expressed sequence Al661453 (Al661453), mRNA [NM_145489]

Mus musculus purine rich element binding protein B (Purb), mRNA [NM_011221]

Mus musculus coxsackie virus and adenovirus receptor (Cxadr), transcript variant 1, mRNA [NM_0010251! Mus musculus a disintegrin-like and metallopeptidase (reprolysin type) with thrombospondin type 1 motif, 5 Mus musculus INO80 homolog (S. cerevisiae) (Ino80), mRNA [NM_026574]

Mus musculus insulin receptor (Insr), mRNA [NM_010568]

Mus musculus 10 days embryo whole body cDNĀ, RIKEN full-length enriched library, clone:2600006K01 pI BC009829 Sin3A associated protein p30-like $\{$ Homo sapiens $\}$ (exp=-1; wgp=0; cg=0), complete [TC161034 Mus musculus SPEG complex locus (Speg), transcript variant 2, mRNA [NM_001085370] Mus musculus nicotinamide nucleotide transhydrogenase (Nnt), transcript variant 1, mRNA [NM_008710] Mus musculus exostoses (multiple)-like 2 (Extl2), transcript variant 1, mRNA [NM_021388] Mus musculus striatin, calmodulin binding protein (Strn), mRNA [NM_011500] Mus musculus armadillo repeat containing 6 (Armc6), mRNA [NM_133972] Mus musculus glutaminyl-peptide cyclotransferase (glutaminyl cyclase) (Qpct), mRNA [NM_027455] Mus musculus aryl hydrocarbon receptor nuclear translocator 2 (Arnt2), mRNA [NM_007488] PREDICTED: Mus musculus uncharacterized LOC102633020 (LOC102633020), ncRNA [XR_382000] 
Mus musculus dispatched homolog 2 (Drosophila) (Disp2), mRNA [NM_170593]

Mus musculus 0 day neonate eyeball cDNA, RIKEN full-length enriched library, clone:E130101M22 produc Mus musculus NME/NM23 nucleoside diphosphate kinase 6 (Nme6), mRNA [NM_018757]

PREDICTED: Mus musculus RIKEN cDNA 3830406C13 gene (3830406C13Rik), transcript variant X1, mR Mus musculus prolylcarboxypeptidase (angiotensinase C) (Prcp), mRNA [NM_028243]

Mus musculus DNA segment, Chr 16, ERATO Doi 472, expressed (D16Ertd472e), transcript variant 2, mRI coiled-coil domain containing 107 [Source:MGI Symbol;Acc:MGI:1913423] [ENSMUST00000107922]

Mus musculus ribonuclease, RNase K (Rnasek), mRNA [NM_173742]

Mus musculus vacuolar protein sorting 4a (yeast) (Vps4a), mRNA [NM_126165]

Mus musculus STT3, subunit of the oligosaccharyltransferase complex, homolog B (S. cerevisiae) (Stt3b), Mus musculus solute carrier family 4, sodium bicarbonate cotransporter, member 7 (Slc4a7), mRNA [NM C Mus musculus ezrin (Ezr), mRNA [NM_009510]

Mus musculus predicted gene 14326 (Gm14326), transcript variant 2, mRNA [NM_001282028]

Mus musculus 12 days embryo male wolffian duct includes surrounding region cDNA, RIKEN full-length en predicted gene 9949 [Source:MGI Symbol;Acc:MGI:3647947] [ENSMUST00000067743]

Mus musculus caspase 7 (Casp7), mRNA [NM_007611]

Mus musculus post-GPI attachment to proteins 2 (Pgap2), transcript variant 1, mRNA [NM_001291358]

Mus musculus StAR-related lipid transfer (START) domain containing 6 (Stard6), transcript variant 1, mRN, Mus musculus zinc finger protein 74 (Zfp74), mRNA [NM_178384]

Mus musculus dual adaptor for phosphotyrosine and 3-phosphoinositides 1 (Dapp1), mRNA [NM_011932] Mus musculus zinc finger protein 868 (Zfp868), transcript variant 1, mRNA [NM_172754]

Mus musculus zyg-II family member B, cell cycle regulator (Zyg11b), mRNA [NM_001033634]

Mus musculus regulatory factor $X$-associated ankyrin-containing protein (Rfxank), transcript variant 1 , mRN Mus musculus cysteine-serine-rich nuclear protein 1 (Csrnp1), mRNA [NM_153287] Mus musculus KDEL (Lys-Asp-Glu-Leu) containing 1 (Kdelc1), mRNA [NM_023645] Mus musculus vacuolar protein sorting 33A (yeast) (Vps33a), mRNA [NM 029929] Mus musculus coiled-coil domain containing 144B (Ccdc144b), mRNA [NM_178418] Mus musculus alpha tubulin acetyltransferase 1 (Atat1), transcript variant 1, mRNA [NM_001142744] Mus musculus 3-oxoacyl-ACP synthase, mitochondrial (Oxsm), mRNA [NM_027695]

Mus musculus glycerol kinase (Gyk), transcript variant 1, mRNA [NM_008194]

Mus musculus D17Mia10e clone c7 mRNA, partial sequence. [U49252]

Mus musculus neuroepithelial cell transforming gene 1 (Net1), transcript variant 1, mRNA [NM_019671] Mus musculus DNA segment, Chr 16, ERATO Doi 472, expressed (D16Ertd472e), transcript variant 1, mRI Mus musculus potassium channel, subfamily K, member 2 (Kcnk2), transcript variant 2, mRNA [NM_01060 Mus musculus regulator of microtubule dynamics 3 (Rmdn3), mRNA [NM_001033136] Mus musculus galanin receptor 2 (Galr2), mRNA [NM_010254]

Mus musculus N(alpha)-acetyltransferase 30, NatC catalytic subunit (Naa30), mRNA [NM_001081430] Mus musculus Rho guanine nucleotide exchange factor (GEF) 39 (Arhgef39), mRNA [NM_001013377] Mus musculus src homology three (SH3) and cysteine rich domain (Stac), mRNA [NM_016853] Mus musculus prostate stem cell antigen (Psca), mRNA [NM_028216] Mus musculus testis expressed 33 (Tex33), transcript variant 1, mRNA [NM_001163612] Mus musculus adult male testis cDNA, RIKEN full-length enriched library, clone:4930439M07 product:uncle Mus musculus FERM domain containing 4A (Frmd4a), transcript variant 2, mRNA [NM_001177843]

Mus musculus tubulin, delta 1 (Tubd1), transcript variant 1, mRNA [NM_001199045]

Mus musculus ribokinase (Rbks), mRNA [NM_153196]

Mus musculus suppressor of Ty 16 (Supt16), mRNA [NM_033618]

Mus musculus zinc finger protein 882 (Zfp882), mRNA [NM_001166645]

Mus musculus netrin G1 (Ntng1), transcript variant a, mRNĀ [NM_030699]

Mus musculus dehydrogenase/reductase (SDR family) X chromosome (Dhrsx), mRNA [NM_001033326]

Mus musculus 0 day neonate head cDNA, RIKEN full-length enriched library, clone:4833419G08 product:u Mus musculus tripartite motif-containing 16 (Trim16), mRNA [NM_053169]

Mus musculus Emx2 opposite strand/antisense transcript (non-protein coding) (Emx2os), long non-coding I Mus musculus antizyme inhibitor 1 (Azin1), transcript variant 2, mRNA [NM_018745] tripartite motif-containing 5 [Source:MGI Symbol;Acc:MGI:3646853] [ENSMŪUST00000138557] Mus musculus ADAMTS-like 2 (Adamtsl2), mRNA [NM 029981] Mus musculus WD repeat domain 52 (Wdr52), mRNA [NM_001033247] Mus musculus amyloid beta (A4) precursor protein-binding, family B, member 2 (Apbb2), transcript variant Mus musculus zinc finger protein 931 (Zfp931), mRNA [NM_001162922] Mus musculus predicted gene 14326 (Gm14326), transcript variant 1, mRNA [NM_001190302] 
Mus musculus platelet-derived growth factor, C polypeptide (Pdgfc), mRNA [NM_019971]

PREDICTED: Mus musculus predicted gene 7967 (Gm7967), misc_RNA [XR_387291]

Mus musculus triosephosphate isomerase 1 (Tpi1), mRNA [NM_009415]

Mus musculus ubiquitin specific peptidase 36 (Usp36), mRNA [N̄M_001033528]

Mus musculus transforming growth factor, beta receptor associated protein 1 (Tgfbrap1), mRNA [NM_0010

Mus musculus hormonally upregulated Neu-associated kinase (Hunk), mRNA [NM_015755]

Mus musculus MLX interacting protein (Mlxip), transcript variant 2, mRNA [NM_133917]

Mus musculus tropomyosin 1, alpha (Tpm1), transcript variant 1, mRNA [NM_001164248]

Mus musculus mannosidase, beta A, lysosomal (Manba), mRNA [NM_027288]

Mus musculus insulin receptor (Insr), mRNA [NM 010568]

Mus musculus MIF4G domain containing (Mif4gd), transcript variant 3, mRNA [NM_001243586]

Mus musculus potassium voltage gated channel, Shaw-related subfamily, member 1 (Kcnc1), transcript val Mus musculus cDNA sequence BC100451 (BC100451), mRNA [NM_021440]

Mus musculus coiled-coil domain containing 65 (Ccdc65), mRNA [NM_153518]

Mus musculus OTU domain containing 7A (Otud7a), mRNA [NM_130880]

Mus musculus cleavage stimulation factor, 3' pre-RNA, subunit 3 (Cstf3), transcript variant 1, mRNA [NM_1 Mus musculus 2-oxoglutarate and iron-dependent oxygenase domain containing 1 (Ogfod1), transcript vari Mus musculus RIKEN cDNA 2610305D13 gene (2610305D13Rik), mRNA [NM_145078]

Mus musculus F-box protein 3 (Fbxo3), transcript variant 2, mRNA [NM_020593]

Mus musculus SMAD family member 7 (Smad7), mRNA [NM_001042660]

Mus musculus collagen, type XII, alpha 1 (Col12a1), mRNA [NM_001290308]

insulin-like growth factor binding protein 3 [Source:MGI Symbol;Acc:MGI:96438] [ENSMUST00000135887]

Mus musculus migration and invasion inhibitory protein (Miip), mRNA [NM_001025365]

Mus musculus family with sequence similarity 45 , member A (Fam45a), transcript variant 1, mRNA [NM_02 Mus musculus p21 protein (Cdc42/Rac)-activated kinase 6 (Pak6), transcript variant 1, mRNA [NM_00103: Mus musculus tumor necrosis factor receptor superfamily, member $1 \mathrm{~b}$ (Tnfrsf1b), mRNA [NM_011610] Mus musculus RIKEN cDNA 2010107G23 gene (2010107G23Rik), mRNA [NM_027251]

Mus musculus predicted pseudogene 5523 (Gm5523), non-coding RNA [NR 004447]

Mus musculus asparagine-linked glycosylation 8 (alpha-1,3-glucosyltransferase) (Alg8), mRNA [NM_19903 Mus musculus single stranded DNA binding protein 4 (Ssbp4), mRNA [NM_133772]

Mus musculus RIKEN cDNA 1500015A07 gene (1500015A07Rik), long non-coding RNA [NR_029432]

Mus musculus transforming growth factor, beta 3 (Tgfb3), mRNA [NM_009368]

Mus musculus transmembrane protein 62 (Tmem62), mRNA [NM_175285]

Mus musculus UDP-Gal:betaGIcNAc beta 1,4-galactosyltransferase, polypeptide 4 (B4galt4), transcript var Mus musculus RAB4A, member RAS oncogene family (Rab4a), mRNA [NM_009003]

Mus musculus predicted gene 14325 (Gm14325), mRNA [NM_001024849]

Mus musculus myocyte enhancer factor 2D (Mef2d), mRNA [NM_133665]

Mus musculus transcription elongation factor A (SII) N-terminal and central domain containing 2 (Tceanc2), Mus musculus amylo-1,6-glucosidase, 4-alpha-glucanotransferase (Agl), mRNA [NM_001081326]

Mus musculus fragile X mental retardation, autosomal homolog 2 (Fxr2), mRNA [NM_011814]

$Y$ box protein 2 [Source:MGI Symbol;Acc:MGI:1096372] [ENSMUST00000018698]

Mus musculus inhibitor of DNA binding 1 (Id1), mRNA [NM_010495]

Mus musculus 11 days pregnant adult female ovary and uterus CDNA, RIKEN full-length enriched library, $c$ Mus musculus rcd1 (required for cell differentiation) homolog 1 (S. pombe) (Rqcd1), mRNA [NM_021383]

Mus musculus family with sequence similarity 129, member C (Fam129c), mRNA [NM_001166213]

Mus musculus RNA polymerase II associated protein 1 (Rpap1), transcript variant 1, mRNA [NM_177294] Mus musculus activated spleen cDNA, RIKEN full-length enriched library, clone:F830223B06 product:hypo Mus musculus mitogen-activated protein kinase 1 interacting protein 1-like (Mapk1ip1I), mRNA [NM_17868 Mus musculus integrator complex subunit 10 (Ints10), mRNA [NM_027590]

Mus musculus mitogen-activated protein kinase 8 (Mapk8), mRNA [NM_016700]

Mus musculus ankyrin repeat and LEM domain containing 1 (Ankle1), mRNA [NM_172756]

Mus musculus ADAMTS-like 4 (AdamtsI4), mRNA [NM_144899]

Mus musculus jerky (Jrk), mRNA [NM_008415]

Mus musculus vacuolar protein sorting 13B (yeast) (Vps13b), mRNA [NM_177151]

PREDICTED: Mus musculus predicted gene 6410 (Gm6410), misc_RNA [XR_378684]

Mus musculus predicted gene 14305 (Gm14305), mRNA [NM_001099327]

Mus musculus insulin receptor (Insr), mRNA [NM_010568]

Mus musculus trafficking protein particle complex 5 (Trappc5), mRNA [NM_025701]

Mus musculus tropomyosin 1, alpha (Tpm1), transcript variant 1, mRNA [NM_001164248] 
Mus musculus neuroepithelial cell transforming gene 1 (Net1), transcript variant 1, mRNA [NM_019671] Mus musculus asparaginyl-tRNA synthetase (Nars), transcript variant 2, mRNA [NM_027350]

Mus musculus protein O-fucosyltransferase 2 (Pofut2), mRNA [NM 030262]

Mus musculus mediator complex subunit 29 (Med29), mRNA [NM_ 026042]

Mus musculus 0 day neonate eyeball cDNA, RIKEN full-length enriched library, clone:E130203P04 product Mus musculus RIKEN cDNA 4930539J05 gene (4930539J05Rik), long non-coding RNA [NR_030689] Mus musculus STT3, subunit of the oligosaccharyltransferase complex, homolog B (S. cerevisiae) (Stt3b), Mus musculus gap junction protein, gamma 2 (Gjc2), transcript variant 2, mRNA [NM_175452]

Mus musculus NME/NM23 nucleoside diphosphate kinase 6 (Nme6), mRNA [NM_018757]

Mus musculus MAD2 mitotic arrest deficient-like 2 (Mad2I2), mRNA [NM_027985]

Mus musculus Kell blood group precursor (McLeod phenotype) homolog (Xk), mRNA [NM_023500]

Mus musculus predicted gene 3893 (Gm3893), long non-coding RNA [NR_033506]

Mus musculus vomeronasal 2, receptor 121 (Vmn2r121), mRNA [NM_001100616]

Mus musculus sine oculis-related homeobox 4 (Six4), mRNA [NM_011382]

Mus musculus mechanistic target of rapamycin (serine/threonine kinase) (Mtor), mRNA [NM_020009]

Mus musculus PET112 homolog (S. cerevisiae) (Pet112), mRNA [NM_144896]

Mus musculus predicted gene 14484 (Gm14484), mRNA [NM_001025260]

Mus musculus vomeronasal 2, receptor 88 (Vmn2r88), mRNA [NM_011686]

Mus musculus RIKEN cDNA 6030419C18 gene (6030419C18Rik), mRNA [NM_176921]

Mus musculus NudC domain containing 3 (Nudcd3), mRNA [NM_173748]

Mus musculus StAR-related lipid transfer (START) domain containing 6 (Stard6), transcript variant 1, mRN, Mus musculus RIKEN cDNA 2410141K09 gene (2410141K09Rik), transcript variant 1, mRNA [NM_00129C Mus musculus haloacid dehalogenase-like hydrolase domain containing 2 (Hdhd2), transcript variant 3, mF Mus musculus cysteinyl leukotriene receptor 1 (Cysltr1), transcript variant 1, mRNA [NM_021476]

Mus musculus nuclear factor of kappa light polypeptide gene enhancer in B cells inhibitor, beta (Nfkbib), ml myosin XVIIIA [Source:MGI Symbol;Acc:MGI:2667185] [ENSMUST00000100795]

Mus musculus predicted gene 10825 (Gm10825), long non-coding RNA [NR_028580]

Mus musculus NADH dehydrogenase (ubiquinone) 1 alpha subcomplex assembly factor 7 (Ndufaf7), mRN, Mus musculus epidermal growth factor receptor pathway substrate 8 (Eps8), transcript variant 1, mRNA [N] PREDICTED: Mus musculus anoctamin 3 (Ano3), transcript variant X3, mRNA [XM_006499262] Mus musculus CREB/ATF bZIP transcription factor (Crebzf), transcript variant 1, mRNA [NM_145151] Mus musculus tubulin, gamma complex associated protein 4 (Tubgcp4), transcript variant 1, mRNA [NM 1! Mus musculus transmembrane protein 181A (Tmem181a), mRNA [NM_001033178]

Mus musculus activated spleen cDNA, RIKEN full-length enriched library, clone:F830048I15 product:hypotl Mus musculus RAB4A, member RAS oncogene family (Rab4a), mRNA [NM_009003]

Mus musculus kin of IRRE like (Drosophila) (Kirrel), transcript variant 1, mRNA [NM_001170985]

Mus musculus EF-hand calcium binding domain 11 (Efcab11), mRNA [NM_030172]

Mus musculus ceramide synthase 2 (Cers2), mRNA [NM_029789]

family with sequence similarity 227, member A [Source:MGI Symbol;Acc:MGI:1922979] [ENSMUSTO0000C Mus musculus spermine synthase (Sms), mRNA [NM_009214]

Mus musculus eukaryotic translation initiation factor 3, subunit J1 (Eif3j1), mRNA [NM_144545]

Mus musculus small nuclear ribonucleoprotein N (Snrpn), transcript variant 3, mRNA [NM_001082962]

Mus musculus IQ motif containing GTPase activating protein 2 (Iqgap2), mRNA [NM_0277̄11]

Mus musculus transmembrane protein 62 (Tmem62), mRNA [NM_175285]

Mus musculus ferrochelatase (Fech), mRNA [NM_007998]

Mus musculus 3 days neonate thymus cDNA, RIKEN full-length enriched library, clone:A630006J12 produc Mus musculus L-2-hydroxyglutarate dehydrogenase (L2hgdh), mRNA [NM_145443]

Mus musculus sulfatase modifying factor 2 (Sumf2), mRNA [NM_026445]

Mus musculus RIKEN cDNA 1700104L18 gene (1700104L18Rik), long non-coding RNA [NR_108033] Mus musculus staufen (RNA binding protein) homolog 1 (Drosophila) (Stau1), transcript variant 3, mRNA [ 1 Mus musculus contactin 6 (Cntn6), mRNA [NM_017383]

Mus musculus synaptic vesicle glycoprotein 2c (Sv2c), mRNA [NM_029210]

Mus musculus valosin containing protein (Vcp), mRNA [NM_009503]

Mus musculus 13 days embryo stomach cDNA, RIKEN full-length enriched library, clone:D530006I24 prodı Mus musculus biogenesis of lysosomal organelles complex-1, subunit 6, pallidin (Bloc1s6), mRNA [NM_01 Mus musculus transmembrane and coiled-coil domains 6 (Tmco6), mRNA [NM_028036] Mus musculus RIKEN cDNA 4933406C10 gene (4933406C10Rik), long non-coding RNA [NR_044986] Mus musculus glucosaminyl ( $\mathrm{N}$-acetyl) transferase 2, I-branching enzyme (Gcnt2), transcript variant 3, mRI Mus musculus fibroblast growth factor receptor 2 (Fgfr2), transcript variant 1, mRNA [NM_010207] 
Mus musculus cDNA sequence BC026513, mRNA (cDNA clone IMAGE:4909841), partial cds. [BC039787] Mus musculus 13 days embryo head cDNA, RIKEN full-length enriched library, clone:3110040I23 product:F Mus musculus WW, C2 and coiled-coil domain containing 1 (Wwc1), mRNA [NM_170779] Mus musculus ataxin 2 (Atxn2), mRNA [NM 009125] Mus musculus RAB8A, member RAS oncogene family (Rab8a), mRNA [NM_023126] PREDICTED: Mus musculus RIKEN cDNA G630016G05 gene (G630016G05Rik), transcript variant X2, mi Mus musculus zinc finger protein 868 (Zfp868), transcript variant 1, mRNA [NM_172754] Mus musculus coenzyme Q5 homolog, methyltransferase (yeast) (Coq5), mRNA [NM_026504] leucine zipper, putative tumor suppressor 1 [Source:MGI Symbol;Acc:MGI:2684762] [ENSMUST00000185 Mus musculus C-type lectin domain family 2, member f (Clec2f), mRNA [NM 001277202] eukaryotic translation initiation factor 2 alpha kinase 4 [Source:MGI Symbol;Acc:MGI:1353427] [ENSMUST Mus musculus coenzyme Q5 homolog, methyltransferase (yeast) (Coq5), mRNA [NM_026504] sideroflexin 5 [Source:MGI Symbol;Acc:MGI:2137681] [ENSMUST00000138489] Mus musculus apolipoprotein O (Apoo), transcript variant 2, mRNA [NM_001199337] Mus musculus cathepsin O (Ctso), mRNA [NM_177662] Mus musculus MAX gene associated (Mga), transcript variant 1, mRNA [NM_013720] Mus musculus nicotinamide riboside kinase 1 (Nmrk1), mRNA [NM_145497] Mus musculus 2 days neonate sympathetic ganglion cDNA, RIKEN full-length enriched library, clone:71204 PREDICTED: Mus musculus predicted gene 5785 (Gm5785), mRNA [XM_974939] Mus musculus RIKEN cDNA 2900026A02 gene (2900026A02Rik), mRNA [NM_172884] Mus musculus adipogenesis associated Mth938 domain containing (Aamdc), transcript variant 1, mRNA [N Mus musculus lysocardiolipin acyltransferase 1 (Lclat1), transcript variant 1, mRNA [NM_001081071] Mus musculus adult male tongue cDNA, RIKEN full-length enriched library, clone:2310007J06 product:uncl Mus musculus predicted gene 14325 (Gm14325), mRNA [NM_001024849] Mus musculus transmembrane protein 18 (Tmem18), mRNA [NM_172049] Mus musculus leucine carboxyl methyltransferase 2 (Lcmt2), mRNA A [NM 177846] Mus musculus transcription elongation factor $\mathrm{A}$ (SII) N-terminal and central domain containing 2 (Tceanc2), Mus musculus churchill domain containing 1 (Churc1), mRNA [NM 206534] Mus musculus lysine $(K)$-specific demethylase 4C (Kdm4c), transcript variant 1, mRNA [NM_144787] Mus musculus adult inner ear cDNA, RIKEN full-length enriched library, clone:F930115A20 product:hypoth Mus musculus excision repaiross-complementing rodent repair deficiency, complementation group 8 (Ercc\& Mus musculus 10 days embryo whole body cDNA, RIKEN full-length enriched library, clone:2610312K03 pl immunoglobulin kappa variable 16-104 [Source:MGI Symbol;Acc:MGI:2685913] [ENSMUST00000103323] Q8BSH3_MOUSE (Q8BSH3) 12 days embryo male wolffian duct includes surrounding region cDNA, RIKE| Mus musculus solute carrier organic anion transporter family, member 5A1 (SIco5a1), mRNA [NM_172841. Mus musculus solute carrier family 30 (zinc transporter), member 7 (Slc30a7), mRNA [NM_023214] Mus musculus serine/threonine kinase 38 (Stk38), mRNA [NM_134115] T cell receptor alpha variable 13N-3 [Source:MGI Symbol;Acc:MGI:3645598] [ENSMUST00000179580] Mus musculus asparagine-linked glycosylation 2 (alpha-1,3-mannosyltransferase) (Alg2), mRNA [NM_019؛ Mus musculus RCB-1283 B16 melanoma cDNA, RIKEN full-length enriched library, clone:G430107I07 pror Mus musculus dynein, axonemal, heavy chain 7A (Dnah7a), mRNA [NM_001252070]

Mus musculus acetyl-Coenzyme A carboxylase beta (Acacb), mRNA [NM_ 133904$]$

Mus musculus adult male bone cDNA, RIKEN full-length enriched library, clone:9830143P17 product:uncla Mus musculus coiled-coil domain containing 88C (Ccdc88c), mRNA [NM_026681]

Mus musculus predicted gene 14499 (Gm14499), mRNA [NM_001277184]

Mus musculus zinc finger and BTB domain containing 34 (Zbtb34), transcript variant 1, mRNA [NM_00108? Mus musculus myosin, light chain 12A, regulatory, non-sarcomeric (Myl12a), mRNA [NM_026064] REM2 and RAB-like small GTPase 1 [Source:MGI Symbol;Acc:MGI:1923416] [ENSMUST̄00000097813] Mus musculus vomeronasal 2, receptor 86 (Vmn2r86), mRNA [NM_001103365] Mus musculus vomeronasal 2, receptor 10 (Vmn2r10), mRNA [NM_009491] Mus musculus predicted gene 5132 (Gm5132), mRNA [NM_001085517] osteopetrosis associated transmembrane protein 1 [Source:MGI Symbol;Acc:MGI:2655574] [ENSMUST00। Mus musculus Emx2 opposite strand/antisense transcript (non-protein coding) (Emx2os), long non-coding I Mus musculus zinc fingers and homeoboxes 2 (Zhx2), mRNA [NM_199449] Mus musculus patched domain containing 2 (Ptchd2), mRNA [NM_001083342] Mus musculus Fas apoptotic inhibitory molecule 2 (Faim2), transcript variant 1, mRNA [NM_028224] Mus musculus cyclin B1 interacting protein 1 (Ccnb1ip1), mRNA [NM_001111119] Mus musculus adenosine deaminase-like (Adal), transcript variant 1, mRNA [NM_029475] Mus musculus TBC1 domain family, member 5 (Tbc1d5), transcript variant 1, mRNA [NM_001285991] 
Mus musculus lung RCB-0558 LLC cDNA, RIKEN full-length enriched library, clone:G730040L14 product:h Mus musculus 15 days embryo head cDNA, RIKEN full-length enriched library, clone:4022436C10 product: Mus musculus citrate lyase beta like (Clybl), mRNA [NM_029556]

Mus musculus predicted gene 13298 (Gm13298), mRNA_[NM_001085530]

Mus musculus CD84 antigen (Cd84), transcript variant 1, mRNA [NM_013489]

Mus musculus glutamate receptor, ionotropic, NMDA2B (epsilon 2) (Grin2b), mRNA [NM_008171]

Mus musculus ankyrin repeat and IBR domain containing 1 (Ankib1), transcript variant 2, mRNA [NM_0010 Mus musculus DEAD (Asp-Glu-Ala-Asp) box polypeptide 51 (Ddx51), mRNA [NM_027156]

PREDICTED: Mus musculus RIKEN cDNA 2810407A14 gene (2810407A14Rik), transcript variant X1, misi Mus musculus congenital dyserythropoietic anemia, type I (human) (Cdan1), mRNA [NM_026891]

Mus musculus angiopoietin-like 7 (Angptl7), mRNA [NM_001039554]

Mus musculus zinc finger protein 72 (Zfp72), mRNA [NM_001081680]

Mus musculus mesoderm development candidate 1 (Mesdc1), mRNA [NM_030705]

Mus musculus nucleus accumbens associated 2, BEN and BTB (POZ) domain containing (Nacc2), transcri Mus musculus Rho guanine nucleotide exchange factor (GEF) 26 (Arhgef26), mRNA [NM_001081295]

Mus musculus ethanol induced 1 (Etohi1), transcript variant 1, mRNA [NM_001177399]

Mus musculus calpain 15 (Capn15), mRNA [NM_015830]

Mus musculus mitofusin 2 (Mfn2), transcript variant 2, mRNA [NM_133201]

Mus musculus solute carrier family 25, member 37 (SIc25a37), mR̄NA [NM_026331]

Mus musculus glucose phosphate isomerase 1 (Gpi1), mRNA [NM_008155]

Mus musculus cyclin L2 (Ccnl2), mRNA [NM_207678]

Mus musculus glucose phosphate isomerase 1 (Gpi1), mRNA [NM_008155]

Mus musculus kinesin light chain 2 (KIc2), transcript variant 1, mRNA [NM_008451]

Mus musculus homeobox D8 (Hoxd8), transcript variant 1, mRNA [NM_008276]

Mus musculus CREB/ATF bZIP transcription factor (Crebzf), transcript variant 1, mRNA [NM_145151]

Mus musculus 4-nitrophenylphosphatase domain and non-neuronal SNAP25-like protein homolog 1 (C. ele Mus musculus membrane associated guanylate kinase, WW and PDZ domain containing 1 (Magi1), transc Mus musculus PHD finger protein 13 (Phf13), mRNA [NM_172705]

Mus musculus ribonuclease P 14 subunit (Rpp14), mRNA [NM_025938]

Mus musculus importin 11 (Ipo11), mRNA [NM_029665]

Mus musculus LanC lantibiotic synthetase component C-like 3 (bacterial) (Lancl3), mRNA [NM_173414]

Mus musculus potassium voltage-gated channel, subfamily $\mathrm{Q}$, member 5 (Kcnq5), transcript variant 1 , mRI Mus musculus mRNA for mKIAA0996 protein. [AK129256]

Mus musculus RAB37, member RAS oncogene family (Rab37), transcript variant 2, mRNA [NM_00116375 Mus musculus predicted gene 5382 (Gm5382), mRNA [NM_001034100]

Mus musculus microfibrillar-associated protein 3 (Mfap3), transcript variant 1, mRNA [NM_145426]

Mus musculus predicted gene 14137 (Gm14137), mRNA [NM_001039223]

Mus musculus heat shock factor binding protein 1-like 1 (Hsbp1l1), mRNA [NM_001136181]

Mus musculus desumoylating isopeptidase 1 (Desi1), mRNA [NM_134095]

Mus musculus toll-like receptor 1 (TIr1), transcript variant 1, mRNA [NM_030682]

Mus musculus adult male diencephalon cDNA, RIKEN full-length enriched library, clone:9330167E06 prodi Mus musculus Rho guanine nucleotide exchange factor (GEF) 38 (Arhgef38), mRNA [NM_029953]

Mus musculus adult male olfactory brain cDNA, RIKEN full-length enriched library, clone:6430528A12 prod Mus musculus ubiquitin-conjugating enzyme E2E 1 (Ube2e1), mRNA [NM_009455]

Mus musculus SR-related CTD-associated factor 8 (Scaf8), mRNA [NM_134123]

Mus musculus adenylate cyclase 6 (Adcy6), mRNA [NM_007405]

Mus musculus expressed sequence AI593442 (AI593442), transcript variant 1, mRNA [NM_001286641]

Mus musculus phosphatidic acid phosphatase type 2A (Ppap2a), transcript variant 1, mRNA [NM_008247]

Mus musculus nuclear fragile $X$ mental retardation protein interacting protein 1 (Nufip1), mRNA [NM_01374 Mus musculus transmembrane protein 135 (Tmem135), mRNA [NM_028343]

Mus musculus RIKEN cDNA 1700012L04 gene (1700012L04Rik), mRNA [NM_029588]

Mus musculus SEC63-like (S. cerevisiae) (Sec63), mRNA [NM_153055]

Mus musculus RAS-like, estrogen-regulated, growth-inhibitor (Rerg), transcript variant 1, mRNA [NM_0011

Mus musculus USO1 vesicle docking factor (Uso1), mRNA [NM_019490]

Mus musculus guanosine diphosphate (GDP) dissociation inhibitor 2 (Gdi2), mRNA [NM_008112]

Mus musculus vomeronasal 2, receptor 16 (Vmn2r16), mRNA [NM_001104627]

Mus musculus NADH dehydrogenase (ubiquinone) 1, subcomplex unknown, 2 (Ndufc2), mRNA [NM_0242.

Mus musculus RAD23b homolog (S. cerevisiae) (Rad23b), mRNA [NM_009011]

UI-M-GI0-cek-h-17-0-UI.r1 NIH_BMAP_GI0 Mus musculus cDNA clone IMAGE: 6840738 5', mRNA sequer 
Mus musculus ATP synthase, $\mathrm{H}+$ transporting mitochondrial F1 complex, beta subunit (Atp5b), mRNA [NM Mus musculus phosphoglycerate mutase 1 (Pgam1), mRNA [NM_023418]

Mus musculus adult male testis cDNA, RIKEN full-length enriched library, clone:4921515G04 product:uncle Mus musculus UDP-N-acetyl-alpha-D-galactosamine:polypeptide $\mathrm{N}$-acetylgalactosaminyltransferase 9 ( $\mathrm{Ga}$ Mus musculus Kell blood group precursor (McLeod phenotype) homolog (Xk), mRNA [NM_023500] Mus musculus RAB37, member RAS oncogene family (Rab37), transcript variant 2, mRNA [NM_00116375 Mus musculus jagunal homolog 1 (Drosophila) (Jagn1), transcript variant 1, mRNA [NM_026365]

Mus musculus natriuretic peptide type A (Nppa), mRNA [NM_008725]

Mus musculus mitochondrial ribosomal protein L34 (Mrpl34), mRNA [NM_053162]

PREDICTED: Mus musculus predicted gene, 20764 (Gm20764), mRNA [XM_003688790]

Mus musculus multivesicular body subunit 12A (Mvb12a), mRNA [NM 028617]

Mus musculus family with sequence similarity 114, member A2 (Fam114a2), transcript variant 2, mRNA [NI PREDICTED: Mus musculus predicted gene 3693 (Gm3693), transcript variant X2, misc_RNA [XR_38175! Mus musculus superkiller viralicidic activity 2-like 2 (S. cerevisiae) (Skiv2l2), mRNA [NM_028151]

Mus musculus protein phosphatase 1, regulatory (inhibitor) subunit 3E (Ppp1r3e), mRNA [NM_001167908] Mus musculus TNFRSF1A-associated via death domain (Tradd), mRNA [NM_001033161]

Mus musculus ankyrin repeat and sterile alpha motif domain containing 1B (Anks $1 \mathrm{~b}$ ), transcript variant 4, $\mathrm{m}$ Mus musculus abhydrolase domain containing 5 (Abhd5), mRNA [NM_026179]

Mus musculus ribosomal protein L36 (Rpl36), mRNA [NM_018730]

Mus musculus trafficking protein particle complex 5 (Trappc5), mRNA [NM 025701]

Mus musculus RIKEN cDNA 2810408A11 gene (2810408A11Rik), mRNA [NM_027419]

Mus musculus G protein-coupled receptor 158 (Gpr158), mRNA [NM_001004761]

Mus musculus spastic paraplegia 11 (Spg11), mRNA [NM_145531]

Mus musculus ribosomal protein L36 (Rpl36), mRNA [NM_018730]

PREDICTED: Mus musculus predicted gene 6404 (Gm6404), mRNA [XM_006517804]

Mus musculus RAB3 GTPase activating protein subunit 2 (Rab3gap2), mRNA [NM 001163754]

Mus musculus THO complex 7 homolog (Drosophila) (Thoc7), transcript variant 3, mRNA [NM_001285780

Mus musculus piggyBac transposable element derived 5 (Pgbd5), mRNA [NM_171824]

Mus musculus bone morphogenetic protein 8a (Bmp8a), transcript variant 1, mRNA [NM_001256019]

Mus musculus ribosomal protein L13A (Rpl13a), mRNA [NM_009438]

Mus musculus ubiquitin specific peptidase 37 (Usp37), mRNA [NM_176972]

Mus musculus SHC (Src homology 2 domain containing) transforming protein 2 (Shc2), mRNA [NM_00102 Mus musculus DNA cross-link repair 1C, PSO2 homolog (S. cerevisiae) (Dclre1c), transcript variant 2, mRI Mus musculus neuroguidin, EIF4E binding protein (Ngdn), mRNA [NM_026890]

Mus musculus folliculin (FIcn), transcript variant 2, mRNA [NM 146018]

PREDICTED: Mus musculus zinc finger protein 74 (Zfp74), transcript variant X1, mRNA [XM_006540384]

Mus musculus caspase 3 (Casp3), transcript variant 2, mRNA [NM_009810]

Mus musculus protocadherin beta 7 (Pcdhb7), mRNA [NM_053132]

Mus musculus EFR3 homolog B (S. cerevisiae) (Efr3b), mRNA [NM_001082483]

Mus musculus Ras interacting protein 1 (Rasip1), mRNA [NM_028544]

Mus musculus tubulin, beta 6 class V (Tubb6), mRNA [NM_026473]

Mus musculus $G$ protein-coupled receptor 135 (Gpr135), mRNA [NM_181752]

Mus musculus REC8 meiotic recombination protein (Rec8), mRNA [NM_020002]

Mus musculus SAP30-like (Sap30l), mRNA [NM_001081168]

Mus musculus nucleotide binding protein-like (Nubpl), mRNA [NM_029760]

Mus musculus chemokine (C-C motif) ligand 28 (Ccl28), mRNA [NM_020279]

Mus musculus SLIT and NTRK-like family, member 3 (Slitrk3), mRNA [NM_198864]

Mus musculus unconventional SNARE in the ER 1 homolog (S. cerevisiae) (Use1), transcript variant 3, mR Mus musculus $\mathrm{G}$ patch domain containing 1 (Gpatch1), mRNA [NM_026181]

Mus musculus F-box and leucine-rich repeat protein 21 (Fbxl21), mRNA [NM_178674]

Mus musculus SIVA1, apoptosis-inducing factor (Siva1), transcript variant 1, mRNA [NM_013929]

Mus musculus small $G$ protein signaling modulator 1 (Sgsm1), transcript variant 3, mRNA [NM_001162965

Mus musculus RIKEN cDNA 1700049G17 gene (1700049G17Rik), mRNA [NM_028538]

Mus musculus family with sequence similarity 160, member B1 (Fam160b1), mRNA [NM_145505]

Mus musculus mitogen-activated protein kinase kinase kinase kinase 1 (Map4k1), mRNA [NM_008279]

Mus musculus RIKEN cDNA 6330416G13 gene (6330416G13Rik), mRNA [NM_144905]

Mus musculus coiled-coil domain containing 71 (Ccdc71), mRNA [NM_133744]

Mus musculus monoglyceride lipase (Mgll), transcript variant 1, mRNA [NM_001166251]

leucine-rich and death domain containing [Source:MGI Symbol;Acc:MGI:1889507] [ENSMUST0000013806 
Mus musculus phosphopantothenoylcysteine decarboxylase (Ppcdc), mRNA [NM_176831]

Mus musculus RIKEN cDNA D130040H23 gene (D130040H23Rik), mRNA [NM_172491]

Mus musculus small integral membrane protein 7 (Smim7), mRNA [NM_172396]

Mus musculus thioredoxin-like 4A (Txnl4a), transcript variant 1, mRNA [NM_025299]

synaptosomal-associated protein 23 [Source:MGI Symbol;Acc:MGI:109356] [ENSMUST00000110711]

Mus musculus N-acetyltransferase 9 (GCN5-related, putative) (Nat9), mRNA [NM_025400]

Mus musculus pleckstrin homology domain containing, family A member 7 (Plekha7), mRNA [NM_172743]

Mus musculus RIKEN cDNA 4930563E22 gene (4930563E22Rik), mRNA [NM_001163728]

Mus musculus myelin basic protein (Mbp), transcript variant 7, mRNA [NM_010777]

Mus musculus prion protein (Prnp), transcript variant 2, mRNA [NM_001278256]

Mus musculus mutS homolog 3 (E. coli) (Msh3), mRNA [NM_010829]

Mus musculus TBC1 domain family, member 2 (Tbc1d2), mRNA [NM_198664]

Mus musculus 6 days neonate head cDNA, RIKEN full-length enriched library, clone:5430410E06 product:I zinc finger protein 81 [Source:MGI Symbol;Acc:MGI:1890752] [ENSMUST00000054072]

Mus musculus slingshot homolog 1 (Drosophila) (Ssh1), mRNA [NM_198109]

Mus musculus Sh3 domain YSC-like 1 (Sh3yl1), transcript variant 1, mRNA [NM_013709]

Mus musculus spastin (Spast), transcript variant 2, mRNA [NM_016962]

Mus musculus RAB26, member RAS oncogene family (Rab26), mRNA [NM_177375]

Mus musculus F-box protein 6 (Fbxo6), transcript variant 1, mRNA [NM_015797]

Mus musculus MEF2 activating motif and SAP domain containing transcriptional regulator (Mamstr), mRN/ Mus musculus patatin-like phospholipase domain containing 3 (Pnpla3), mRNA [NM_054088]

Mus musculus serine hydroxymethyltransferase 1 (soluble) (Shmt1), mRNA [NM_009171]

PREDICTED: Mus musculus predicted pseudogene 7241 (Gm7241), mRNA [XM_006543539]

Mus musculus solute carrier organic anion transporter family, member $2 b 1$ (SIco2b1), transcript variant 1, $n$ Mus musculus 12 days embryo spinal ganglion cDNA, RIKEN full-length enriched library, clone:D130094KC Mus musculus RIKEN cDNA 4930570G19 gene (4930570G19Rik), transcript variant 2, long non-coding RA Mus musculus wingless-type MMTV integration site 9A (Wnt9a), mRNA [NM_139298]

Mus musculus transmembrane protein 192 (Tmem192), transcript variant 1, mRNA [NM_028427]

Mus musculus leucine rich repeat containing 73 (Lrrc73), mRNA [NM_001111142]

Mus musculus serine peptidase inhibitor, Kazal type 2 (Spink2), transcript variant 2, mRNA [NM_183284]

Mus musculus oxytocin receptor (Oxtr), mRNA [NM_001081147]

Mus musculus transmembrane emp24 protein transport domain containing 4 (Tmed4), mRNA [NM_13402C

Mus musculus cytochrome $c$ oxidase assembly factor 4 (Coa4), mRNA [NM_183270]

Mus musculus glutathione S-transferase kappa 1 (Gstk1), mRNA [NM_029555]

Mus musculus tripartite motif-containing 12C (Trim12c), transcript variant 1, mRNA [NM_001146007]

Mus musculus CDP-diacylglycerol synthase 1 (Cds1), mRNA [NM_173370]

Mus musculus fibroblast activation protein (Fap), mRNA [NM_007986]

Mus musculus nodal modulator 1 (Nomo1), mRNA [NM_153057]

Mus musculus adult male urinary bladder cDNA, RIKEN full-length enriched library, clone:9530073P05 pro Mus musculus T cell activation GTPase activating protein 1 (Tagap1), mRNA [NM_147155]

Mus musculus BCL2-like 12 (proline rich) (Bcl2112), mRNA [NM_029410]

Mus musculus immunoglobulin superfamily, member 10 (Igsf10), mRNA [NM_001162884]

Mus musculus high mobility group box transcription factor 1 (Hbp1), transcript variant 2, mRNA [NM_1779s

Mus musculus NEL-like 1 (Nell1), mRNA [NM_001037906]

Mus musculus synergin, gamma (Synrg), mRNA [NM_194341]

Mus musculus protein phosphatase 2 (formerly $2 A$ ), regulatory subunit B", delta (Ppp2r3d), transcript varial Mus musculus putative homeodomain transcription factor 1 (Phtf1), transcript variant 1, mRNA [NM_01362 Mus musculus progestin and adipoQ receptor family member VI (Paqr6), mRNA [NM_198410] Mus musculus tRNA splicing endonuclease 54 homolog (S. cerevisiae) (Tsen54), mRNA [NM_029557] PREDICTED: Mus musculus predicted gene 6306 (Gm6306), mRNA [XM_006500499]

PREDICTED: Mus musculus predicted gene 5093 (Gm5093), mRNA [XM_006543383]

Mus musculus RAS-like, family 10, member A (Rasl10a), mRNA [NM_145216]

Mus musculus SURP and G patch domain containing 1 (Sugp1), mRNA [NM_027481]

Mus musculus DEAD (Asp-Glu-Ala-Asp) box polypeptide 55 (Ddx55), transcript variant 1, mRNA [NM_026، Mus musculus ubiquitin specific peptidase 53 (Usp53), mRNA [NM_133857]

Mus musculus adult male testis cDNA, RIKEN full-length enriched library, clone:4930522L14 product:simile Mus musculus coiled-coil domain containing 91 (Ccdc91), mRNA [NM_025911]

Mus musculus oligonucleotide/oligosaccharide-binding fold containing 1 (Obfc1), mRNA [NM_175360]

Mus musculus cytokine receptor-like factor 2 (Crlf2), transcript variant 1, mRNA [NM_001164735] 
Mus musculus centromere protein K (Cenpk), transcript variant 1, mRNA [NM_021790] Mus musculus zinc finger protein 608 (Zfp608), mRNA [NM_175751]

Mus musculus 3 days neonate thymus cDNA, RIKEN full-length enriched library, clone:A630055C05 produ Mus musculus solute carrier family 10 (sodium/bile acid cotransporter family), member 3 (Slc10a3), transcr Mus musculus Holliday junction recognition protein (Hjurp), mRNA [NM_198652]

Mus musculus D-tyrosyl-tRNA deacylase 2 (Dtd2), mRNA [NM 029545]

Mus musculus progestin and adipoQ receptor family member VI (Paqr6), mRNA [NM_198410]

Mus musculus palmdelphin (Palmd), mRNA [NM_023245]

Mus musculus ferritin light chain 1 (Ftl1), mRNA [NM_010240]

Mus musculus THAP domain containing 6 (Thap6), non-coding RNA [NR 028429]

PREDICTED: Mus musculus uncharacterized LOC102634598 (LOC102634598), misc_RNA [XR_386205]

Mus musculus monoglyceride lipase (Mgll), transcript variant 1, mRNA [NM_001166251]

Mus musculus RIKEN cDNA 1810009N02 gene (1810009N02Rik), mRNA [NM 026939]

Mus musculus solute carrier family 25, member 41 (SIc25a41), mRNA [NM_175333]

Mus musculus aldo-keto reductase family 1, member C19 (Akr1c19), mRNA [NM_001013785]

Mus musculus transmembrane protein 173 (Tmem173), transcript variant 1, mRNA [NM_028261]

Mus musculus ubiquitin C (Ubc), mRNA [NM_019639]

predicted gene 10654 [Source:MGI Symbol;Acc:MGI:3643366] [ENSMUST00000098653]

Mus musculus histocompatibility 2, M region locus 3 (H2-M3), mRNA [NM_013819]

Mus musculus NIM1 serine/threonine protein kinase (Nim1k), mRNA [NM_175538]

Mus musculus beaded filament structural protein 2, phakinin (Bfsp2), mRNA [NM_001002896]

Mus musculus alkB, alkylation repair homolog 6 (E. coli) (Alkbh6), mRNA [NM_198027]

Mus musculus CaM kinase-like vesicle-associated (Camkv), mRNA [NM_145621]

Mus musculus polymerase (DNA directed), iota (Poli), transcript variant 2 , mRNA [NM_011972]

Mus musculus zinc finger protein 566 (Zfp566), mRNA [NM_152814]

Mus musculus retinol binding protein 7, cellular (Rbp7), mRNA [NM_022020]

PREDICTED: Mus musculus predicted gene 8013 (Gm8013), transcript variant X4, ncRNA [XR_389350]

Mus musculus synaptogyrin 2 (Syngr2), mRNA [NM_009304]

Mus musculus LSM4 homolog, U6 small nuclear RNA associated (S. cerevisiae) (Lsm4), mRNA [NM_0158

Mus musculus FXYD domain-containing ion transport regulator 6 (Fxyd6), mRNA [NM_022004]

Mus musculus solute carrier family 2 (facilitated glucose transporter), member 9 (SIc2a9), transcript variant Mus musculus SERTA domain containing 3 (Sertad3), mRNA [NM_133210]

Mus musculus acyl-CoA synthetase long-chain family member 5 (Acsl5), mRNA [NM_027976]

Mus musculus SLIT and NTRK-like family, member 5 (Slitrk5), mRNA [NM_198865]

cut-like homeobox 2 [Source:MGI Symbol;Acc:MGI:107321] [ENSMUST00000111752]

BEN domain containing 7 [Source:MGI Symbol;Acc:MGI:2443100] [ENSMUST00000115022]

Mus musculus ES cells cDNA, RIKEN full-length enriched library, clone:C330018M05 product:similar to ZF Mus musculus myosin, light polypeptide 2, regulatory, cardiac, slow (Myl2), mRNA [NM_010861]

Mus musculus telomeric repeat binding factor 1 (Terf1), transcript variant 1, mRNA [NM_009352]

Mus musculus predicted gene 13247 (Gm13247), transcript variant 1, mRNA [NM_001243138]

Mus musculus polymerase (DNA directed), epsilon (Pole), mRNA [NM_011132]

Mus musculus Holliday junction recognition protein (Hjurp), mRNA [NM_198652]

Mus musculus coiled-coil domain containing 91 (Ccdc91), mRNA [NM_025911]

Mus musculus G2/M-phase specific E3 ubiquitin ligase (G2e3), transcript variant 2, mRNA [NM_00116796: Mus musculus PTPRF interacting protein, binding protein 2 (liprin beta 2) (Ppfibp2), transcript variant 1, mF Mus musculus methyltransferase like 3 (Mettl3), mRNA [NM_019721]

Mus musculus thyroid hormone responsive (Thrsp), mRNA [NM_009381]

Mus musculus 9.5 days embryo parthenogenote cDNA, RIKEN full-length enriched library, clone:B130019[ Mus musculus 16 days neonate thymus cDNA, RIKEN full-length enriched library, clone:A130072N09 prod Mus musculus CD1d2 antigen (Cd1d2), transcript variant 1, mRNA [NM 007640]

Mus musculus phosphatidylinositol glycan anchor biosynthesis, class $\mathrm{P}$ (Pigp), transcript variant 5 , mRNA [ Mus musculus germ cell-specific gene 2 (Gsg2), mRNA [NM_010353]

Mus musculus huntingtin interacting protein K (Hypk), mRNA [NM_026318]

Mus musculus apolipoprotein L 7b (Apol7b), mRNA [NM_001024848]

Mus musculus apoptotic chromatin condensation inducer 1 (Acin1), transcript variant 2, mRNA [NM_02319

Mus musculus methyltransferase like 17 (Mett117), mRNA [NM_001029990]

Mus musculus Friend virus susceptibility 1 (Fv1), mRNA [NM_010244]

Mus musculus churchill domain containing 1 (Churc1), mRNA [NM_206534]

Mus musculus predicted gene 15698 (Gm15698), non-coding RNA [NR_003564] 
Mus musculus microsomal triglyceride transfer protein (Mttp), transcript variant 1, mRNA [NM_001163457]

Mus musculus adrenergic receptor kinase, beta 2 (Adrbk2), transcript variant 1, mRNA [NM_177078]

Mus musculus RIKEN cDNA 4930515G01 gene (4930515G01Rik), long non-coding RNA [NR_027872] ribosomal protein S3A3 [Source:MGI Symbol;Acc:MGI:3643406] [ENSMUST00000074680]

Mus musculus Ca2+-dependent secretion activator (Cadps), transcript variant 1, mRNA [NM_012061]

Mus musculus RIKEN cDNA 2410006H16 gene (2410006H16Rik), long non-coding RNA [NR_030738]

Mus musculus RWD domain containing 3 (Rwdd3), transcript variant 2, mRNA [NM_028456]

Mus musculus cortistatin (Cort), mRNA [NM_007745]

Mus musculus kinesin light chain 4 (KIc4), mRNA [NM_029091]

Mus musculus G2/M-phase specific E3 ubiquitin ligase (G2e3), transcript variant 3, mRNA [NM 00116796، Mus musculus 4HAUS augmin-like complex, subunit 8 (Haus8), transcript variant 1, mRNA [NM_00116304 Mus musculus EH-domain containing 4 (Ehd4), mRNA [NM_133838]

Mus musculus interleukin 17 receptor D (II17rd), mRNA [NM 134437] regulating synaptic membrane exocytosis 3 [Source:MGI Symbol;Acc:MGI:2443331] [ENSMUST00000071 Mus musculus RAB19, member RAS oncogene family (Rab19), mRNA [NM_011226]

Mus musculus FXYD domain-containing ion transport regulator 2 (Fxyd2), transcript variant b, mRNA [NM_ Mus musculus creatine kinase, mitochondrial 1, ubiquitous (Ckmt1), mRNA [NM_009897]

Mus musculus dehydrogenase/reductase (SDR family) member 7 (Dhrs7), mRNA [NM_025522]

Mus musculus zinc finger protein 286 (Zfp286), mRNA [NM 138949]

Mus musculus polymerase (DNA directed), kappa (Polk), mRNA [NM_012048]

Mus musculus cysteine-rich PDZ-binding protein (Cript), mRNA [NM_019936]

mp78f05.y1 Soares_thymus_2NbMT Mus musculus cDNA clone IMAGE:575361 5'. [AI530666]

Mus musculus $G$ protein-coupled receptor 180 (Gpr180), mRNA [NM_021434]

Mus musculus 11 days pregnant adult female ovary and uterus cDNA, RIKEN full-length enriched library, $\mathrm{C}$ Mus musculus neuron-glia-CAM-related cell adhesion molecule (Nrcam), transcript variant 1, mRNA [NM_1 Mus musculus RIKEN cDNA 2810459M11 gene (2810459M11Rik), transcript variant 1, mRNA [NM 00114 Mus musculus asparaginase homolog (S. cerevisiae) (Aspg), mRNA [NM_001081169]

Mus musculus uncharacterized LOC100862268 (LOC100862268), transcript variant 1, long non-coding RN Mus musculus DEAD (Asp-Glu-Ala-Asp) box polypeptide 4 (Ddx4), transcript variant 2, mRNA [NM_01002؛ Mus musculus ATPase, class I, type 8B, member 5 (Atp8b5), mRNA [NM_177195] Mus musculus dynein, axonemal, intermediate chain 2 (Dnaic2), mRNA [NM_001034878] Mus musculus kelch repeat and BTB (POZ) domain containing 11 (Kbtbd11), mRNA [NM_029116] Mus musculus nudix (nucleoside diphosphate linked moiety X)-type motif 7 (Nudt7), transcript variant 4, mF Mus musculus predicted gene 7120 (Gm7120), transcript variant 2, mRNA [NM_001177666] Mus musculus adult male medulla oblongata cDNA, RIKEN full-length enriched library, clone:6330442E02 Mus musculus Iroquois related homeobox 1 (Drosophila) (Irx1), mRNA [NM_010573] Mus musculus ribosomal protein L34, pseudogene 1 (Rpl34-ps1), mRNA [NM_001199350] Mus musculus doublecortin domain containing 2a (Dcdc2a), transcript variant 1, mRNA [NM_177577] Mus musculus parathyroid hormone 1 receptor (Pth1r), transcript variant 1, mRNA [NM_011199] Mus musculus synaptotagmin IX (Syt9), mRNA [NM_021889] Mus musculus glutathione S-transferase, mu 6 (Gstm6), mRNA [NM 008184] Mus musculus gem (nuclear organelle) associated protein 5 (Gemin $\overline{5}$ ), transcript variant 1, mRNA [NM_00. Mus musculus RUN and FYVE domain containing 3 (Rufy3), transcript variant 3, mRNA [NM_001289776] Q8NFW1_HUMAN (Q8NFW1) Alpha 1 type XXII collagen, partial (33\%) [TC1681647]

Mus musculus protein tyrosine phosphatase, receptor type, $R$ (Ptprr), transcript variant 1, mRNA [NM_011。 Mus musculus microtubule-associated protein 1S (Map1s), mRNA [NM_173013] Mus musculus cDNA sequence BC005764 (BC005764), transcript variant 1, mRNA [NM_181681] Mus musculus zinc finger protein 536 (Zfp536), mRNA [NM_172385]

Mus musculus RALBP1 associated Eps domain containing protein 2 (Reps2), transcript variant 1, mRNA [ $\mathrm{N}$ Mus musculus family with sequence similarity 83, member G (Fam83g), mRNA [NM_178618]

Mus musculus guanine nucleotide binding protein, alpha 14 (Gna14), mRNA [NM_008137]

Mus musculus teashirt zinc finger family member 1 (Tshz1), mRNA [NM_001081300]

Mus musculus adult male testis cDNA, RIKEN full-length enriched library, clone:4930532K22 product:hypo Mus musculus RIKEN cDNA 4930515G01 gene (4930515G01Rik), long non-coding RNA [NR_027872] Mus musculus oxysterol binding protein-like 10 (Osbpl10), mRNA [NM_148958] Mus musculus periaxin (Prx), transcript variant 2, mRNA [NM_019412] Mus musculus Rap guanine nucleotide exchange factor (GEF) 3 (Rapgef3), transcript variant 2, mRNA [NN Mus musculus Rho GTPase activating protein 22 (Arhgap22), mRNA [NM_153800] Mus musculus RIKEN cDNA 2010109A12 gene (2010109A12Rik), mRNA [NM_029363] 
Mus musculus carbonyl reductase 3 (Cbr3), mRNA [NM_173047]

BB713741 RIKEN full-length enriched, 2 cells egg Mus musculus cDNA clone B020049A06 3', mRNA sequ predicted gene 6816 [Source:MGI Symbol;Acc:MGI:3648347] [ENSMUST00000059737] Mus musculus transmembrane protein 65 (Tmem65), mRNA [NM_175212]

Mus musculus pleckstrin homology domain containing, family B (evectins) member 1 (Plekhb1), transcript I Mus musculus histamine receptor H1 (Hrh1), transcript variant 1, mRNA [NM_001252643]

PREDICTED: Mus musculus ADP-ribosylation factor 1-like (LOC102632770), misc_RNA [XR_399117] Mus musculus M-phase phosphoprotein 10 (U3 small nucleolar ribonucleoprotein) (Mphosph10), mRNA [N Mus musculus activated spleen cDNA, RIKEN full-length enriched library, clone:F830106C12 product:hypo Mus musculus centromere protein K (Cenpk), transcript variant 1, mRNA [NM_021790] histone cluster 3, H2ba [Source:MGI Symbol;Acc:MGI:1925553] [ENSMUST00000078267] Mus musculus cation channel, sperm associated 2 (Catsper2), mRNA [NM_153075] Mus musculus microtubule-associated protein 1 A (Map1a), transcript variant 1, mRNA [NM_032393] Mus musculus TRAF-interacting protein (Traip), mRNA [NM_011634]

Mus musculus protein tyrosine phosphatase, non-receptor type 5 (Ptpn5), transcript variant 1, mRNA [NM Mus musculus seven in absentia homolog 3 (Drosophila) (Siah3), mRNA [NM_001128093]

Mus musculus heparanase (Hpse), mRNA [NM_152803]

Mus musculus iduronidase, alpha-L- (Idua), transcript variant 1, mRNA [NM_008325]

Mus musculus predicted gene 2696 (Gm2696), mRNA [NM_001205009]

Mus musculus mannan-binding lectin serine peptidase 2 (Masp2), transcript variant 2, mRNA [NM_010767. Mus musculus ferritin light chain 1 (Ftl1), mRNA [NM_010240]

Mus musculus bisphosphate 3'-nucleotidase 1 (Bpnt1), mRNA [NM_011794]

Mus musculus synaptoporin (Synpr), transcript variant 2, mRNA [NM_001163032]

Mus musculus synuclein, gamma (Sncg), mRNA [NM_011430]

PREDICTED: Mus musculus uncharacterized LOC102634215 (LOC102634215), ncRNA [XR_386425]

Mus musculus H2A histone family, member J (H2afj), mRNA [NM 177688]

Mus musculus coiled-coil domain containing 184 (Ccdc184), mRNA [NM_177716]

Mus musculus transmembrane emp24 protein transport domain containing 5 (Tmed5), mRNA [NM $02887 €$ Mus musculus solute carrier family 6 (neurotransmitter transporter), member 17 (SIc6a17), mRNA [NM_17: Mus musculus carbohydrate ( $\mathrm{N}$-acetylgalactosamine 4-0) sulfotransferase 14 (Chst14), mRNA [NM_02811 Mus musculus C-type lectin domain family 16, member A (Clec16a), transcript variant 1, mRNA [NM_1775 Mus musculus GATA zinc finger domain containing 2A (Gatad2a), transcript variant 1, mRNA [NM_ 145596 Mus musculus inositol 1,4,5-trisphosphate 3-kinase A (Itpka), mRNA [NM_146125]

Mus musculus synaptojanin 2 (Synj2), transcript variant 1, mRNA [NM_001113353]

Mus musculus RIKEN cDNA 2310045N01 gene (2310045N01Rik), mRNA [NM_001145552]

Mus musculus L-3-hydroxyproline dehydratase (trans-) (L3hypdh), mRNA [NM_026038]

Mus musculus UbiA prenyltransferase domain containing 1 (Ubiad1), mRNA [NM_027873]

Mus musculus integrin beta 4 (Itgb4), transcript variant 1, mRNA [NM_001005608]

v-erb-a erythroblastic leukemia viral oncogene homolog 4 (avian) [Source:MGI Symbol;Acc:MGI:104771] [E Mus musculus solute carrier family 27 (fatty acid transporter), member 3 (SIc27a3), mRNA [NM_011988] Mus musculus HscB iron-sulfur cluster co-chaperone homolog (E. coli) (Hscb), mRNA [NM_153571] Mus musculus solute carrier family 48 (heme transporter), member 1 (SIc48a1), mRNA [NM_026353] Mus musculus meiotic nuclear divisions 1 homolog (S. cerevisiae) (Mnd1), mRNA [NM_029797] Mus musculus alcohol dehydrogenase, iron containing, 1 (Adhfe1), transcript variant 1, mRNA [NM_175231 Mus musculus solute carrier family 35, member E1 (SIc35e1), mRNA [NM_177766] Mus musculus late endosomal/lysosomal adaptor, MAPK and MTOR activator 3 (Lamtor3), mRNA [NM_01 Mus musculus a disintegrin-like and metallopeptidase (reprolysin type) with thrombospondin type 1 motif, 1 Mus musculus Holliday junction recognition protein (Hjurp), mRNA [NM_198652]

Mus musculus fatty acid binding protein 5, epidermal (Fabp5), transcript variant 2, mRNA [NM_001272097] Mus musculus calmodulin binding transcription activator 1 (Camta1), transcript variant 1, mRNA [NM_0010 Mus musculus RIKEN cDNA E330013P04 gene (E330013P04Rik), long non-coding RNA [NR_026942] Mus musculus ChaC, cation transport regulator 1 (Chac1), mRNA [NM_026929]

Mus musculus selenium binding protein 1 (Selenbp1), mRNA [NM_009150]

Mus musculus protocadherin beta 4 (Pcdhb4), mRNA [NM_053129]

Mus musculus solute carrier family 13 (sodium-dependent citrate transporter), member 5 (Slc13a5), mRNA Mus musculus 10, 11 days embryo whole body cDNA, RIKEN full-length enriched library, clone:2810451K1 Mus musculus acid phosphatase 1, soluble (Acp1), transcript variant 1, mRNA [NM_001110239] Mus musculus predicted gene 7120 (Gm7120), transcript variant 2, mRNA [NM_001177666] Mus musculus islet cell autoantigen 1-like (Ica1I), mRNA [NM_027407] 
Mus musculus ribonuclease T2A (Rnaset2a), mRNA [NM_001083938]

Mus musculus RIKEN cDNA 1700010114 gene (1700010I14Rik), mRNA [NM_025851]

Mus musculus predicted gene 13157 (Gm13157), mRNA [NM_001127189]

Mus musculus intraflagellar transport 27 (Ift27), mRNA [NM_025931]

Mus musculus RIKEN cDNA 3110070M22 gene (3110070M22Rik), long non-coding RNA [NR_027974]

Mus musculus CD1d1 antigen (Cd1d1), mRNA [NM_007639]

Mus musculus synaptotagmin XV (Syt15), transcript variant a, mRNA [NM_181529]

Mus musculus pannexin 1 (Panx1), mRNA [NM_019482]

Mus musculus small nuclear RNA activating complex, polypeptide 1 (Snapc1), mRNA [NM_178392]

Mus musculus 2 days pregnant adult female ovary cDNA, RIKEN full-length enriched library, clone:E33002

Mus musculus protein kinase, cAMP dependent regulatory, type II beta (Prkar2b), mRNA [NM_011158]

Mus musculus EF-hand calcium binding domain 12 (Efcab12), mRNA [NM_001110506]

Mus musculus olfactomedin-like 3 (Olfml3), mRNA [NM_133859]

Mus musculus lectin, galactose-binding, soluble 2 (Lgals2), mRNA [NM_025622]

Mus musculus cysteine-rich protein 1 (intestinal) (Crip1), mRNA [NM_007763]

Mus musculus Ly6/neurotoxin 1 (Lynx1), mRNA [NM_011838]

Mus musculus transmembrane protein 140 (Tmem140), mRNA [NM_197986]

Mus musculus RIKEN cDNA 4932415M13 gene (4932415M13Rik), long non-coding RNA [NR_073205]

Mus musculus microfibrillar-associated protein 1A (Mfap1a), mRNA [NM_026220]

Mus musculus eukaryotic translation initiation factor 2-alpha kinase 2 (Eif2ak2), mRNA [NM_011163]

Mus musculus RIKEN cDNA 6720483E21 gene (6720483E21Rik), long non-coding RNA [NR_040492]

Mus musculus leukocyte specific transcript 1 (Lst1), mRNA [NM_010734]

Mus musculus malic enzyme 3, NADP(+)-dependent, mitochondrial (Me3), mRNA [NM_181407]

Mus musculus calmodulin binding transcription activator 1 (Camta1), transcript variant 1 , mRNA [NM_0010

Mus musculus guanine nucleotide binding protein (G protein), beta 4 (Gnb4), mRNA [NM_013531]

Mus musculus ribosomal protein S4-like (Rps4I), non-coding RNA [NR_003634]

Mus musculus transmembrane protein 132B (Tmem132b), mRNA [NM_001190352]

Mus musculus a disintegrin-like and metallopeptidase (reprolysin type) with thrombospondin type 1 motif, 1 Mus musculus ATP-binding cassette, sub-family C (CFTR/MRP), member 4 (Abcc4), transcript variant 1, $\mathrm{m}$ Mus musculus malic enzyme 3, NADP(+)-dependent, mitochondrial (Me3), mRNA [NM_181407]

Mus musculus apoptotic chromatin condensation inducer 1 (Acin1), transcript variant 2, mRNA [NM_02319 Mus musculus calcium channel, voltage-dependent, alpha2/delta subunit 3 (Cacna2d3), mRNA [NM_00978 Mus musculus proteasome (prosome, macropain) subunit, beta type 9 (large multifunctional peptidase 2) (F Mus musculus minichromosome maintenance deficient 3 (S. cerevisiae) (Mcm3), mRNA [NM_008563]

Mus musculus solute carrier family 26 (sulfate transporter), member 1 (SIc26a1), mRNA [NM_174870]

Mus musculus solute carrier family 13 (sodium-dependent citrate transporter), member 5 (Slc13a5), mRNA

Mus musculus ring finger protein 43 (Rnf43), mRNA [NM_172448]

Mus musculus zinc finger protein 930 (Zfp930), mRNA [NM_001013379]

Mus musculus solute carrier family 29 (nucleoside transporters), member 4 (SIc29a4), mRNA [NM_146257

Mus musculus ataxia telangiectasia mutated homolog (human) (Atm), mRNA [NM_007499]

Mus musculus purinergic receptor P2X, ligand-gated ion channel, 7 (P2rx7), transcript variant 2, mRNA [NN

Mus musculus strain ICR clone Li(i)01 endogenous retrovirus U3 region, partial sequence. [FJ654066]

Mus musculus meiotic nuclear divisions 1 homolog (S. cerevisiae) (Mnd1), mRNA [NM_029797]

Mus musculus zinc finger homeobox 3 (Zfhx3), mRNA [NM_007496]

Mus musculus 15 days embryo head cDNA, RIKEN full-length enriched library, clone:D930032C08 product Mus musculus RIKEN cDNA 6330403K07 gene (6330403K07Rik), mRNA [NM_134022]

Mus musculus DNA cross-link repair 1C, PSO2 homolog (S. cerevisiae) (Dclre1c), transcript variant 2, mRI Mus musculus RNA polymerase II associated protein 2 (Rpap2), transcript variant 3, mRNA [NM_0011634l Mus musculus inhibitor of four 1 (Mif1) mRNA, complete cds. [DQ459435]

Mus musculus RIKEN cDNA 2010315B03 gene (2010315B03Rik), transcript variant 1, mRNA [NM_00124: Mus musculus zinc finger protein 619 (Zfp619), mRNA [NM_001004139]

Mus musculus olfactomedin-like 1 (Olfml1), mRNA [NM_172907]

Mus musculus RIKEN cDNA A730008H23 gene (A730008H23Rik), mRNA [NM_172505]

Mus musculus dynein regulatory complex subunit 1 (Drc1), mRNA [NM_001033460]

Mus musculus RIKEN cDNA 1600014C10 gene (1600014C10Rik), transcript variant 1, mRNA [NM_00108!

Mus musculus chordin-like 1 (Chrdl1), transcript variant 1, mRNA [NM_001114385]

Mus musculus NLR family, CARD domain containing 5 (NIrc5), mRNA [NM_001033207]

Mus musculus hydroxyprostaglandin dehydrogenase 15 (NAD) (Hpgd), mRNA [NM_008278]

Mus musculus T cell activation Rho GTPase activating protein (Tagap), mRNA [NM_145968] 
Mus musculus immunoglobulin superfamily, member 21 (Igsf21), mRNA [NM_198610]

Mus musculus growth differentiation factor 15 (Gdf15), mRNA [NM_011819]

Mus musculus potassium large conductance calcium-activated channel, subfamily $\mathrm{M}$, beta member 4 (Kcnr Mus musculus BH3 interacting domain death agonist (Bid), mRNA [NM_007544]

Mus musculus chloride channel Kb (Clcnkb), mRNA [NM_019701]

Mus musculus fibulin 2 (Fbln2), transcript variant 1, mRNA [NM 007992]

Mus musculus LIM domain and actin binding 1 (Lima1), transcript variant a, mRNA [NM_001113545]

Mus musculus lung RCB-0558 LLC cDNA, RIKEN full-length enriched library, clone:G730026111 product:uI Mus musculus 12 days embryo male wolffian duct includes surrounding region cDNA, RIKEN full-length en Mus musculus cDNA clone IMAGE:40090117. [BC128469]

Mus musculus 2 days pregnant adult female ovary cDNA, RIKEN full-length enriched library, clone:E33003 Mus musculus tubulin, beta 3 class III (Tubb3), mRNA [NM_023279]

Mus musculus potassium voltage-gated channel, Shal-related family, member 2 (Kcnd2), mRNA [NM 0196 Mus musculus adult male cecum cDNA, RIKEN full-length enriched library, clone:9130022E09 product:hyp Mus musculus CDC42 effector protein (Rho GTPase binding) 1 (Cdc42ep1), mRNA [NM_027219] Mus musculus myosin, heavy polypeptide 1, skeletal muscle, adult (Myh1), mRNA [NM_030679] PREDICTED: Mus musculus predicted gene, 20746 (Gm20746), mRNA [XM_006542883] Mus musculus small nucleolar RNA host gene 6 (Snhg6), long non-coding RN̄A [NR_024067] Mus musculus ring finger protein 41 (Rnf41), transcript variant 2, mRNA [NM_026259] Mus musculus A kinase (PRKA) anchor protein 5 (Akap5), mRNA [NM_001101471] Mus musculus membrane-associated ring finger (C3HC4) 8 (March8), mRNA [NM_027920] Mus musculus RNA polymerase II associated protein 3 (Rpap3), mRNA [NM_028003] Mus musculus UDP-N-acetyl-alpha-D-galactosamine:polypeptide N-acetylgalactosaminyltransferase 15 (G Mus musculus N-myristoyltransferase 2 (Nmt2), transcript variant 1, mRNA [NM_008708] Mus musculus 13 days embryo whole body cDNA, RIKEN full-length enriched library, clone:3930401B19 pI Mus musculus 2-phosphoxylose phosphatase 1 (Pxylp1), transcript variant 1, mRNA [NM 001289645] Mus musculus mitochondrial ribosomal protein S12 (Mrps12), mRNA [NM_011885] Mus musculus transmembrane protein 254a (Tmem254a), transcript variant 1, mRNA [NM 026679] Mus musculus adult male urinary bladder cDNA, RIKEN full-length enriched library, clone:9530030H05 pro Mus musculus 4 days neonate male adipose cDNA, RIKEN full-length enriched library, clone:B430105A11 Mus musculus dynein light chain LC8-type 1 (Dynll1), mRNA [NM_019682]

Mus musculus chemokine (C-C motif) ligand 25 (Ccl25), transcript variant 2, long non-coding RNA [NR_03: Mus musculus transmembrane protein 178 (Tmem178), mRNA [NM 026516]

Mus musculus cell adhesion molecule with homology to L1CAM (Chl1), mRNA [NM_007697]

Mus musculus transmembrane protein 254c (Tmem254c), transcript variant 1, mRNA [NM 001270498] Mus musculus lipoma HMGIC fusion partner-like 3 (Lhfpl3), transcript variant 2, mRNA [NM_001081231] Mus musculus adult male hippocampus cDNA, RIKEN full-length enriched library, clone:2900019G23 prodı Mus musculus adult male hippocampus cDNA, RIKEN full-length enriched library, clone:2900019G23 prodı Mus musculus BMP2 inducible kinase (Bmp2k), mRNA [NM_080708]

Mus musculus leucine rich repeat and sterile alpha motif containing 1 (Lrsam1), mRNA [NM_199302] Mus musculus collagen, type IV, alpha 6 (Col4a6), mRNA [NM_053185] Mus musculus serine/threonine kinase receptor associated protein (Strap), mRNA [NM_011499] Mus musculus purine-nucleoside phosphorylase 2 (Pnp2), mRNA [NM_001123371] Mus musculus dehydrogenase/reductase (SDR family) member 7C (Dhrs7c), mRNA [NM_001013013] Mus musculus RIKEN cDNA A930005H10 gene (A930005H10Rik), transcript variant 2, long non-coding RI Mus musculus pituitary tumor-transforming gene 1 (Pttg1), transcript variant 2, mRNA [NM_013917] Mus musculus anaphase-promoting complex subunit 5 (Anapc5), transcript variant 1, mRNA [NM_021505] PREDICTED: Mus musculus RIKEN cDNA A030001D20 gene (A030001D20Rik), misc_RNA [XR_105107] Mus musculus stathmin-like 3 (Stmn3), mRNA [NM_009133]

Mus musculus $\mathrm{S} 100$ calcium binding protein, zeta (Ŝ0z), mRNA [NM_001081159]

Mus musculus LIM domain only 1 (Lmo1), mRNA [NM_057173]

Mus musculus tissue inhibitor of metalloproteinase 4 (Timp4), mRNA [NM_080639]

Mus musculus integrin alpha 9 (Itga9), transcript variant 1, mRNA [NM_133721]

Mus musculus complement component 2 (within H-2S) (C2), mRNA [NM_013484]

Mus musculus EH-domain containing 3 (Ehd3), mRNA [NM_020578]

Mus musculus potassium intermediate/small conductance calcium-activated channel, subfamily $\mathrm{N}$, membel Mus musculus doubl homeobox B-like 2 (Duxbl2), mRNA [NM_001177538]

Mus musculus lectin, galactose binding, soluble 4 (Lgals4), mRNA [NM_010706]

Mus musculus lectin, galactose binding, soluble 4 (Lgals4), mRNA [NM_010706] 
Mus musculus GATA zinc finger domain containing 2A (Gatad2a), transcript variant 1, mRNA [NM_145596 Mus musculus nuclear receptor subfamily 1, group D, member 2, mRNA (cDNA clone MGC:106146 IMAGE Mus musculus F-box protein 17 (Fbxo17), mRNA [NM_015796]

Mus musculus glutamate receptor, metabotropic 5 (Grm5), transcript variant b, mRNA [NM 001143834] Mus musculus ribosomal protein S3A1 (Rps3a1), mRNA [NM_016959] Mus musculus endothelial cell-specific molecule 1 (Esm1), mRNA [NM_023612] Mus musculus RIKEN cDNA 4833420G17 gene (4833420G17Rik), transcript variant 1, mRNA [NM_02612 Mus musculus nicotinamide nucleotide adenylyltransferase 3 (Nmnat3), mRNA [NM_144533] Mus musculus exonuclease 3'-5' domain containing 1 (Exd1), mRNA [NM_172857] Q3JOL9_RHOS4 (Q3JOL9) Hydrogenase maturation factor F, partial (3\%) [TC1616199]

Mus musculus cell adhesion molecule with homology to L1CAM (ChI1), mRNA [NM_007697]

Mus musculus protein arginine N-methyltransferase 8 (Prmt8), mRNA [NM_201371]

Mus musculus KN motif and ankyrin repeat domains 1 (Kank1), mRNA [NM 181404]

Mus musculus hydroxyprostaglandin dehydrogenase 15 (NAD) (Hpgd), mRN̄A [NM_008278]

Mus musculus leucine rich repeat and sterile alpha motif containing 1 (Lrsam1), mRNA [NM_199302]

Mus musculus collagen, type II, alpha 1 (Col2a1), transcript variant 2, mRNA [NM_001113515]

Mus musculus growth associated protein 43 (Gap43), mRNA [NM_008083]

Mus musculus translocase of outer mitochondrial membrane 22 homolog (yeast) (Tomm22), mRNA [NM_1 Mus musculus adult male bone cDNA, RIKEN full-length enriched library, clone:9830138L07 product:uncla: Mus musculus histocompatibility 2, Q region locus 5 (H2-Q5), non-coding RNA [NR_051981]

Mus musculus pyruvate dehydrogenase (lipoamide) beta (Pdhb), mRNA [NM_024221]

Mus musculus amyloid beta (A4) precursor protein-binding, family A, member 2 (Apba2), transcript variant Mus musculus stathmin-like 4 (Stmn4), mRNA [NM_019675]

Mus musculus Ly6/neurotoxin 1 (Lynx1), mRNA [NM_011838]

Mus musculus butyrylcholinesterase (Bche), mRNA [NM_009738]

Mus musculus SH3-domain GRB2-like 3 (Sh3gl3), transcript variant 1, mRNA [NM 017400]

Mus musculus uncoupling protein 3 (mitochondrial, proton carrier) (Ucp3), mRNA [NM_009464]

Mus musculus stathmin-like 4 (Stmn4), mRNA [NM 019675]

Mus musculus peptidyl arginine deiminase, type II (Padi2), mRNA [NM_008812]

Mus musculus $G$ protein-coupled receptor, family C, group 5, member $\bar{A}$ (Gprc5a), mRNA [NM_181444]

Mus musculus lipoprotein lipase (Lpl), mRNA [NM_008509]

Mus musculus lipoprotein lipase (Lpl), mRNA [NM_008509]

Mus musculus peptidyl arginine deiminase, type II (Padi2), mRNA [NM_008812]

Mus musculus uncoupling protein 2 (mitochondrial, proton carrier) (Ucp2), mRNA [NM_011671]

Mus musculus abhydrolase domain containing 1 (Abhd1), mRNA [NM_021304]

Mus musculus RIKEN cDNA 4833420G17 gene (4833420G17Rik), transcript variant 1, mRNA [NM_02612 Mus musculus serine (or cysteine) peptidase inhibitor, clade A, member 3N (Serpina3n), mRNA [NM_0092. Mus musculus complement component factor i (Cfi), mRNA [NM_007686]

UDP-N-acetyl-alpha-D-galactosamine:polypeptide N-acetylgalactosaminyltransferase 15 [Source:MGI Sym Mus musculus purinergic receptor P2X, ligand-gated ion channel, 7 (P2rx7), transcript variant 1, mRNA [NN Mus musculus phosphatidylethanolamine binding protein 2 (Pbp2), mRNA [NM_029595]

Mus musculus olfactory receptor 224 (Olfr224), mRNA [NM_207695]

Mus musculus processing of precursor 4, ribonuclease P/MRP family, (S. cerevisiae) (Pop4), mRNA [NM_( Mus musculus POU domain, class 6, transcription factor 1 (Pou6f1), mRNA [NM_010127]

Mus musculus protocadherin 17 (Pcdh17), mRNA [NM_001013753]

Mus musculus family with sequence similarity 183, member B (Fam183b), transcript variant 1, mRNA [NM Mus musculus RIKEN cDNA 1810037I17 gene (1810037I17Rik), mRNA [NM_024461]

Mus musculus RIKEN cDNA 1810037 I17 gene (1810037I17Rik), mRNA [NM_024461]

Mus musculus sterile alpha motif domain containing 4 (Samd4), transcript variant 1, mRNA [NM_00103722 Mus musculus serine (or cysteine) peptidase inhibitor, clade A, member 3I (Serpina3i), mRNA [N̄M_00119 Mus musculus collagen, type IV, alpha 6 (Col4a6), mRNA [NM_053185]

Mus musculus XIAP associated factor 1 (Xaf1), transcript variant 1, mRNA [NM_001037713] 


\begin{tabular}{|c|c|c|}
\hline $\operatorname{logFC}$ & AveExpr & adj.P.Val \\
\hline 5.326621277 & 7.971349421 & $1.5155 \mathrm{E}-21$ \\
\hline 4.706882168 & 7.941454285 & $4.18232 \mathrm{E}-23$ \\
\hline 3.159607921 & 8.528529626 & $2.51609 \mathrm{E}-15$ \\
\hline 2.76634258 & 8.199204967 & 1.89631E-15 \\
\hline 2.625034338 & 7.006201591 & $6.07388 \mathrm{E}-14$ \\
\hline 2.57614419 & 6.975926163 & $4.55163 \mathrm{E}-12$ \\
\hline 2.535424208 & 9.5022869 & $5.66539 \mathrm{E}-10$ \\
\hline 2.528377361 & 9.568691036 & $2.68489 \mathrm{E}-12$ \\
\hline 2.4557783 & 8.486798968 & $5.03003 E-15$ \\
\hline 2.227984321 & 11.43046767 & 0.011383754 \\
\hline 2.222558264 & 6.863824084 & 6.99163E-09 \\
\hline 2.221223649 & 7.455657139 & 1.00373E-08 \\
\hline 2.187870963 & 8.102504202 & 1.76297E-14 \\
\hline 2.17951172 & 8.499105459 & 7.34401E-09 \\
\hline 2.155696224 & 9.038758021 & $9.52423 \mathrm{E}-12$ \\
\hline 2.142761169 & 7.714208648 & $2.51609 \mathrm{E}-15$ \\
\hline 2.141524133 & 6.950826515 & $1.26452 \mathrm{E}-13$ \\
\hline 2.123548403 & 7.11520769 & 3.81067E-16 \\
\hline 2.000964933 & 7.331541801 & $1.71376 \mathrm{E}-13$ \\
\hline 1.998798403 & 9.506990359 & 0.000527398 \\
\hline 1.994500273 & 6.927881761 & $1.32742 \mathrm{E}-15$ \\
\hline 1.966656603 & 8.572006753 & $8.45076 \mathrm{E}-09$ \\
\hline 1.91680325 & 9.198570422 & $6.39585 \mathrm{E}-07$ \\
\hline 1.912680866 & 11.20738139 & $6.74098 \mathrm{E}-11$ \\
\hline 1.903303041 & 6.870745089 & $5.0348 \mathrm{E}-13$ \\
\hline 1.832444376 & 8.366008201 & $1.75238 \mathrm{E}-08$ \\
\hline 1.797567531 & 7.969980889 & 1.87675E-06 \\
\hline 1.763559756 & 13.64296854 & 1.62674E-08 \\
\hline 1.750158707 & 9.743132096 & $9.79076 \mathrm{E}-06$ \\
\hline 1.726988441 & 9.069085973 & 0.000151451 \\
\hline 1.725246422 & 12.829438 & 0.002006738 \\
\hline 1.701860019 & 6.732605311 & $1.20027 \mathrm{E}-10$ \\
\hline 1.692648894 & 10.18934601 & $9.90908 \mathrm{E}-05$ \\
\hline 1.680832596 & 8.494281435 & $1.34408 \mathrm{E}-05$ \\
\hline 1.680817626 & 10.32761111 & $1.10036 \mathrm{E}-06$ \\
\hline 1.661805686 & 9.694988353 & 0.00193586 \\
\hline 1.64885182 & 9.034037272 & 246485 \\
\hline 1.602415256 & 8.177045633 & 1.9256E-09 \\
\hline 1.574018429 & 10.33013058 & $72 \mathrm{E}-06$ \\
\hline 1.554543727 & 10.33206306 & 1.77247E-14 \\
\hline 1.5382424 & 11.07205551 & $3.00086 \mathrm{E}-06$ \\
\hline 1.53117695 & 10.52421298 & 0.002874148 \\
\hline 1.517239691 & 7.995104458 & 1.94892E-09 \\
\hline 1.515844412 & 7.480665769 & 0.000172194 \\
\hline 1.506485285 & 9.878089363 & 0.000877619 \\
\hline 1.503395436 & 9.872073547 & 8.60945E-07 \\
\hline 1.501278472 & 10.14705428 & 0.001129742 \\
\hline 1.460709523 & 7.978713091 & 2.92927E-08 \\
\hline 1.408511896 & 15.4731878 & 3.00086E-06 \\
\hline 1.403818102 & 7.624333535 & 6.62656E-06 \\
\hline 1.383573947 & 9.459809864 & 6.70599E-07 \\
\hline 1.382367568 & 11.13618528 & $1.13328 \mathrm{E}-08$ \\
\hline 1.374998141 & 9.208882095 & 7.54693E-07 \\
\hline 1.360071196 & 8.840699972 & $2.13081 \mathrm{E}-07$ \\
\hline 1.288727894 & 8.746606738 & 3.00779E-07 \\
\hline & & \\
\hline
\end{tabular}




\begin{tabular}{|c|c|c|}
\hline & & \\
\hline & & \\
\hline & 11 & \\
\hline 257 & 7.5080 & \\
\hline 9573 & 7.422795425 & $19 \mathrm{E}-0$ \\
\hline 7094651 & 7.59703769 & \\
\hline 25413714 & 11.05468172 & $3.32214 \mathrm{E}-0$ \\
\hline 25400917 & 7.4799979 & 0.001 \\
\hline 247972291 & 7.18748402 & \\
\hline & 7.705143143 & \\
\hline & & \\
\hline & & \\
\hline 1 & $6.6 \varsigma$ & \\
\hline & 11.7 & \\
\hline & 10.7 & \\
\hline & 10.7 & \\
\hline 222 & 6.61 & 1.1 \\
\hline & 9.07 & 0.0 \\
\hline 077 & 10.3 & \\
\hline & & \\
\hline & & \\
\hline & & \\
\hline & & \\
\hline & 9.9 & \\
\hline & 10. & 0.0 \\
\hline & 9.33 & 0.0 \\
\hline & 9.94 & 8.7 \\
\hline 779 & 9.4 & 0.0 \\
\hline & 6.9 & 2.6 \\
\hline & & \\
\hline & & \\
\hline & & \\
\hline & & \\
\hline & 10. & \\
\hline $1.07=$ & & \\
\hline & 11. & \\
\hline 776 & 6.6 & E-06 \\
\hline & 13. & E-07 \\
\hline & 7.5 & \\
\hline & 7.0 & 0.00 \\
\hline & 10. & \\
\hline & & \\
\hline & & \\
\hline & & \\
\hline & & \\
\hline & 7.0 & \\
\hline & 15.3 & E-07 \\
\hline 1.00 & 6.69 & E-06 \\
\hline 1.001 & 8.43 & $\mathrm{E}-08$ \\
\hline & 9.77 & 5445 \\
\hline 0226 & 9.19 & 6641 \\
\hline 253 & 10.4 & 6451 \\
\hline & 7.32 & E-08 \\
\hline & & \\
\hline & 6.42 & \\
\hline & 14.0 & \\
\hline 0.972 & 10.13 & \\
\hline & & \\
\hline & 12.667583 & 0.003856341 \\
\hline
\end{tabular}


1

2

3

4

5

6

7

8

9

10

11

12

13

14

15

16

17

18

19

20

21

22

23

24

25

26

27

28

29

30

31

32

33

34

35

36

37

38

39

40

41

42

43

44

45

46

47

48

49

50

51

52

53

54

55

56

57

58

59

60

\begin{tabular}{|c|c|c|}
\hline & 699007 & \\
\hline & & \\
\hline & 12.01103127 & \\
\hline & & \\
\hline 953245847 & & \\
\hline 952385121 & 6.56148348 & \\
\hline & 9.571912151 & \\
\hline 8888 & 7.16170 & \\
\hline 3743 & 8.5960 & \\
\hline 4699 & 7.9879 & 418551 \\
\hline 7122 & 12.3 & 0.00 \\
\hline 0273 & 9.499 & \\
\hline & 7.015 & \\
\hline & 8.820 & \\
\hline & 9.628 & \\
\hline & $9.57 \mathrm{c}$ & \\
\hline 732 & 10.09 & \\
\hline & & \\
\hline & 7.7967 & 44E-07 \\
\hline & & 2E-08 \\
\hline & 8.85 & \\
\hline & 7.037 & \\
\hline & & \\
\hline & 6.47 & \\
\hline & & \\
\hline & & \\
\hline 687 & 79 & \\
\hline 28 & & \\
\hline & & \\
\hline & & \\
\hline & & \\
\hline & 36 & \\
\hline & 2 & \\
\hline & & \\
\hline & & \\
\hline & & \\
\hline & 47 & \\
\hline 142 & 83 & \\
\hline & & \\
\hline & & \\
\hline & & \\
\hline & & 3E-06 \\
\hline 496 & 186 & \\
\hline & & \\
\hline & & \\
\hline & & \\
\hline & & 51 \\
\hline 915964 & 2077 & 39E-09 \\
\hline & 11.87147738 & \\
\hline & 8.505777767 & 11945 \\
\hline & 7.804274362 & \\
\hline & 7.664091488 & 358E-05 \\
\hline & & $3.29145 \mathrm{E}-05$ \\
\hline & & \\
\hline & 7.115904662 & \\
\hline & 7.747422009 & 0.001299081 \\
\hline & 8.487905687 & \\
\hline & 6.839913205 & $6.44035 \mathrm{E}-0$ \\
\hline 0.809691701 & 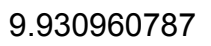 & \\
\hline
\end{tabular}

https://mc04.manuscriptcentral.com/cares 


\begin{tabular}{|c|c|c|}
\hline & & \\
\hline 311273 & 7.553472865 & \\
\hline 302564631 & 572185508 & \\
\hline .79913581 & 11.07024261 & \\
\hline 0.798266378 & 10.21390203 & \\
\hline 796972973. & & \\
\hline & & \\
\hline & & \\
\hline 792549929 & & \\
\hline 791982 & 9522 & \\
\hline 12 & 3654 & \\
\hline 0.789316322 & 8.448857429 & \\
\hline 0.785035418 & 11.98425589 & \\
\hline 0.784649746 & 25413 & 0.0 \\
\hline 0.783418591 & 4978 & \\
\hline 0.782106906 & 4465 & 0.0 \\
\hline 0.776997444 & & \\
\hline 0.774332816 & & \\
\hline 0.772816 & & \\
\hline 0.7719 & & \\
\hline 0.769 & & \\
\hline 0.76926 & 8.2 & \\
\hline 0.7675 & & \\
\hline 0.767356 & 3239 & \\
\hline 0.7660 & 11. & 0.0 \\
\hline 0.76287 & 312 & \\
\hline 0.76068 & & \\
\hline 0.755 & & \\
\hline & & \\
\hline & & \\
\hline 0.74 & & \\
\hline 0.74 & 326 & 0.0 \\
\hline $0.7446 \mathrm{C}$ & 093 & 0.0 \\
\hline 0.73417 & 597 & 0. \\
\hline $0.72 \mathrm{~s}$ & 8.5 & 0.0 \\
\hline 0.72931 & & 23 \\
\hline 0.72 & & 0 \\
\hline & & \\
\hline & & \\
\hline & & \\
\hline & & \\
\hline & & \\
\hline 0.71 & 8.8 & \\
\hline 0.70 & 964 & 0.0 \\
\hline & 609 & 0.0 \\
\hline 0.70 & & \\
\hline 0.70 & 925 & 0.0 \\
\hline 0.69 & & \\
\hline 0.691 & & \\
\hline & & \\
\hline & & \\
\hline & & \\
\hline & & \\
\hline 0.685 & & \\
\hline 0.680 & & 2.4 \\
\hline $0.67927 \varepsilon$ & & 0.00 \\
\hline & & 0.002 \\
\hline & & 0.01540475 \\
\hline & & \\
\hline
\end{tabular}

https://mc04.manuscriptcentral.com/cares 
1

2

3

4

5

6

7

8

9

10

11

12

13

14

15

16

17

18

19

20

21

22

23

24

25

26

27

28

29

30

31

32

33

34

35

36

37

38

39

40

41

42

43

44

45

46

47

48

49

50

51

52

53

54

55

56

57

58

59

60

\begin{tabular}{|c|c|c|}
\hline 0.674397108 & 7.74997714 & \\
\hline & 7.244348901 & \\
\hline & 9.369172174 & \\
\hline 66786877 & 6.852701833 & \\
\hline & & \\
\hline & 8.526 & \\
\hline & 9.312 & \\
\hline & 11.60 & \\
\hline & 8.892 & \\
\hline & 12.5 & \\
\hline & 11.80 & \\
\hline & 14.14 & \\
\hline & 824 & \\
\hline & 9.44 & \\
\hline & 6.92 & \\
\hline & & \\
\hline & 11.0 & \\
\hline & 7.25 & \\
\hline & 8.62 & \\
\hline & 9.28 & \\
\hline & & \\
\hline & 8.98 & \\
\hline & & \\
\hline & & \\
\hline & 8.73 & \\
\hline & & \\
\hline & 8.75 & \\
\hline & & \\
\hline & & \\
\hline & 8.64 & \\
\hline & & \\
\hline & & \\
\hline & & \\
\hline & & \\
\hline & & \\
\hline & 7.16 & 51 \\
\hline & 9.62 & \\
\hline & 8.64 & \\
\hline & & \\
\hline & & \\
\hline & 7.32 & 0.00 \\
\hline & & \\
\hline & & \\
\hline & & \\
\hline & & \\
\hline & 8.53 & \\
\hline & 8.29 & \\
\hline & 8.47 & \\
\hline & & \\
\hline & 6.595 & \\
\hline & 7.155 & \\
\hline & & \\
\hline & 11.9 & \\
\hline & 6.187 & \\
\hline & & \\
\hline & 7.020264708 & 0.01092334 \\
\hline & & \\
\hline & 8.866986993 & 0.02414329 \\
\hline & 17224 & 0.00431750 \\
\hline
\end{tabular}

https://mc04.manuscriptcentral.com/cares 


\begin{tabular}{|c|c|c|}
\hline 594601426 & & \\
\hline 594041539 & 9.155739992 & \\
\hline 593343466 & 12.72558299 & \\
\hline 1697254 & & \\
\hline & & \\
\hline & 1.69706941 & \\
\hline & & \\
\hline & & \\
\hline 2 & & \\
\hline 583352015 & 87 & \\
\hline 0.5807349 & 07 & \\
\hline 0.578759 & 9.2325215 & \\
\hline 0.576549426 & 7.490033464 & 0.046 \\
\hline 5074749 & 7.18473457 & \\
\hline 755514 & 6.807269488 & \\
\hline 0.567 & 11.55214483 & \\
\hline & & \\
\hline & & \\
\hline & & \\
\hline 0.55 & & \\
\hline 0.55 & 11.3 & \\
\hline 0.55 & 20213 & 0.0 \\
\hline 0.55 & 275828 & 450 \\
\hline 0.54 & 484853 & 0.02 \\
\hline 0.54 & 88 & 0.0 \\
\hline & 6.37 & \\
\hline & & \\
\hline & & \\
\hline & & \\
\hline & & \\
\hline & & \\
\hline & $14 . \varepsilon$ & \\
\hline 0.53 & $9.8 \mathrm{c}$ & 54 \\
\hline 28446 & 10.8 & 0.0 \\
\hline 0.53 & 9.17 & 0.0 \\
\hline & 9.11 & \\
\hline & & \\
\hline & 7.2 & \\
\hline & & \\
\hline & & \\
\hline & & \\
\hline & & \\
\hline 0.53 & 39522 & 0.0 \\
\hline 0.52 & 64761 & 0.0 \\
\hline 0.529 & 7.217970946 & 12478 \\
\hline 0.527 & 046652 & 0.00 \\
\hline & & 0.0 \\
\hline 0.526 & 11.42 & 0.03 \\
\hline & & \\
\hline & & \\
\hline 0.525 & & \\
\hline & & \\
\hline & & \\
\hline & & \\
\hline & 6.1661 & $1.95 \varepsilon$ \\
\hline & 7.8646123 & \\
\hline & 7.3237457 & \\
\hline & & \\
\hline & & \\
\hline
\end{tabular}


2

3

4

5

6

7

8

9

10

11

12

13

14

15

16

17

18

19

20

21

22

23

24

25

26

27

28

29

30

31

32

33

34

35

36

37

38

39

40

41

42

43

44

45

46

47

48

49

50

51

52

53

54

55

56

57

58

59

60

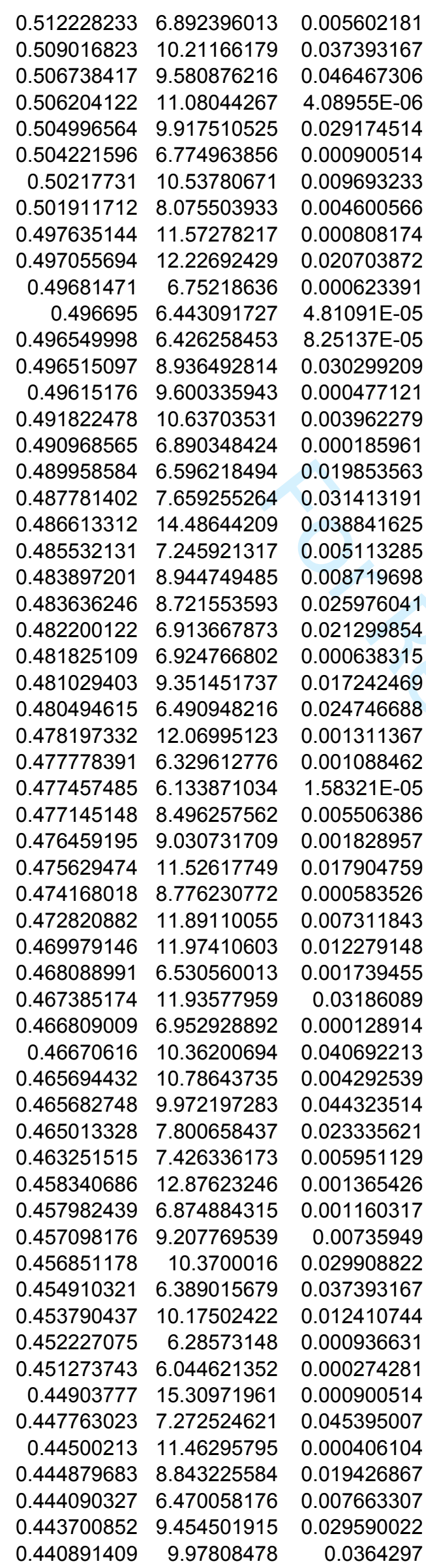

https://mc04.manuscriptcentral.com/cares 


$\begin{array}{rrr}0.440689436 & 6.983094672 & 0.046991837 \\ 0.438953046 & 9.873997921 & 0.045460992 \\ 0.43524986 & 14.53699509 & 0.037393167 \\ 0.427666332 & 13.23524556 & 0.007459508 \\ 0.424137876 & 10.59066735 & 0.008859233 \\ 0.42271889 & 6.292352942 & 0.001815965 \\ 0.42043698 & 6.57986675 & 0.001443363 \\ 0.419476387 & 8.945056584 & 0.000436557 \\ 0.419198928 & 7.577093736 & 0.005240145 \\ 0.41913221 & 6.343168511 & 0.003787885 \\ 0.417164393 & 7.420248711 & 0.00462664 \\ 0.416936983 & 8.769634807 & 0.0265819 \\ 0.416637414 & 7.721938883 & 0.017846136 \\ 0.415844374 & 11.68965048 & 0.04845752 \\ 0.41559322 & 7.125120729 & 0.006593344 \\ 0.415243264 & 8.932685792 & 0.013039063 \\ 0.414414455 & 8.003857616 & 0.024855151 \\ 0.413708974 & 6.218528632 & 0.002123539 \\ 0.413684181 & 8.461838113 & 0.005754049 \\ 0.412405535 & 6.947356535 & 0.046775339 \\ 0.411471092 & 11.92703754 & 0.045134321 \\ 0.409296709 & 9.949768884 & 0.017753758 \\ 0.408617418 & 6.306220274 & 0.012271924 \\ 0.406099667 & 10.10361514 & 0.032432537 \\ 0.40553698 & 12.25146122 & 0.008704796 \\ 0.405340924 & 10.26010917 & 0.000498551 \\ 0.404316557 & 8.001395018 & 0.022577663 \\ 0.40263635 & 13.35054757 & 0.013939533 \\ 0.402091708 & 10.49093052 & 0.031169026 \\ 0.401581925 & 6.726663181 & 0.002578168 \\ 0.398522876 & 9.191087781 & 0.045460992 \\ 0.397935551 & 6.495699396 & 0.011884866 \\ 0.397025619 & 6.260679891 & 0.00123595 \\ 0.394629866 & 10.55175122 & 0.002065838 \\ 0.394162375 & 6.254228174 & 0.019070637 \\ 0.392889528 & 8.867834981 & 0.002218742 \\ 0.392477146 & 7.244947197 & 0.024070563 \\ 0.392156901 & 6.228406796 & 0.017837447 \\ 0.3917772 & 12.77511628 & 0.023798032 \\ 0.391173241 & 6.982595294 & 0.033268917 \\ 0.39117049 & 6.474571593 & 0.005790588 \\ 0.390395955 & 6.37202715 & 0.003880208 \\ 0.390172821 & 6.442100669 & 0.020221663 \\ 0.389765394 & 6.700200388 & 0.003413735 \\ 0.387386265 & 6.58660119 & 0.038259174 \\ 0.38576947 & 7.042352643 & 0.011625638 \\ 0.385361306 & 16.30849845 & 0.004062968 \\ 0.383686004 & 6.568754731 & 0.024070563 \\ 0.381511625 & 6.091046043 & 0.000980465 \\ 0.379830315 & 6.27031141 & 0.033835141 \\ 0.379563561 & 6.283761675 & 0.001546482 \\ 0.378501009 & 6.99491057 & 0.000999501 \\ 0.376192058 & 6.496326483 & 0.000901307 \\ 0.375666934 & 6.692151172 & 0.031546005 \\ 0.372827738 & 6.5520809 & 0.000700661 \\ 0.36593115 & 6.831524973 & 0.023981552 \\ 0.365251988 & 9.103756239 & 0.00069584 \\ & & \\ 0.374149604 & 0.009147095 \\ 0.015647875\end{array}$


1

2

3

4

5

6

7

8

9

10

11

12

13

14

15

16

17

18

19

20

21

22

23

24

25

26

27

28

29

30

31

32

33

34

35

36

37

38

39

40

41

42

43

44

45

46

47

48

49

50

51

52

53

54

55

56

57

58

59

60

\begin{tabular}{|c|c|c|}
\hline 0.36350122 & 7.161944693 & \\
\hline 0.362276281 & 6.964030088 & \\
\hline 361906265 & 9.542409873 & \\
\hline 232 & 6.680187468 & 0.027 \\
\hline 360506202 & 6.583811787 & \\
\hline 360280168 & 6.359031467 & \\
\hline 50064 & 11.36207192 & $0.02734 \varepsilon$ \\
\hline 77757 & 10.16927723 & 0.045 \\
\hline 156 & 6.040017011 & 0.02 \\
\hline 0.358850754 & 8.473046024 & 0.022807 \\
\hline .357228462 & 6.165392545 & 0.00079 \\
\hline 00342 & 7.847411318 & 0.029 \\
\hline 3908 & 6.76590339 & 0.027 \\
\hline 514 & 15.548 & 0.00 \\
\hline 958 & 12.24174198 & \\
\hline 0.34826 & 7.063109429 & 0.036 \\
\hline 3115 & 6.57454886 & \\
\hline & & \\
\hline 716 & 10.60161225 & 0.02 \\
\hline 554 & 16.14117805 & 0.02 \\
\hline 4589 & 9.599327514 & 0.005 \\
\hline 618 & 15.58858 & \\
\hline & & \\
\hline 328027082 & 6.093584714 & 0.004 \\
\hline 398 & 10.64772 & 0.04 \\
\hline 0.3270 & 11.36759244 & 0.01 \\
\hline 088 & 12.32067837 & \\
\hline & & \\
\hline 4724 & 9.9562 & 0.0 \\
\hline 262 & 12.22021 & 0.0 \\
\hline & & \\
\hline 5093 & 69691 & 0.02 \\
\hline 3076 & 7.10 & \\
\hline 0405 & 6.94 & 0.0 \\
\hline .30857958 & 6.411 & 0.0 \\
\hline & & \\
\hline 0.307841 & 6.2490 & 0.0 \\
\hline 0.305411046 & 6.175418302 & \\
\hline 9921 & 9.223 & 0.04 \\
\hline 0.304755981 & 6.483885608 & 0.03 \\
\hline & & \\
\hline 0.297713631 & 6.022117917 & 0.02 \\
\hline 0.292306122 & 6.846252621 & 0.04 \\
\hline 0.288261272 & 12.4664347 & 0.02 \\
\hline 0.287834928 & 11.40734343 & 0.04 \\
\hline 0.286563466 & 10.60793854 & \\
\hline 0.285949357 & 6.264288781 & 0.02 \\
\hline 0.28340742 & 12.70854687 & 0.026 \\
\hline 0.28340483 & 8.406819665 & \\
\hline 0.282838866 & 10.08696021 & 0.03821566 \\
\hline 0.280394525 & 6.499018685 & \\
\hline 0.279371698 & 9.600818144 & 7380 \\
\hline 0.278174735 & 6.186459568 & 0.03762292 \\
\hline 0.27386441 & 11.79810942 & 0.02940697 \\
\hline 0.268127974 & 15.07008493 & 0.02212601 \\
\hline 0.267919866 & 5.980558817 & 0.02592250 \\
\hline 0.267545274 & 14.98538925 & 0.02903915 \\
\hline 0.266502634 & 11.43165245 & 0.01439407 \\
\hline 0.265694877 & 5.93789424 & \\
\hline
\end{tabular}

https://mc04.manuscriptcentral.com/cares 


\begin{tabular}{|c|c|c|}
\hline & & \\
\hline 6250287 & .53968412 & \\
\hline 261423553 & 071820881 & \\
\hline 0.260345438 & 6.151726529 & \\
\hline 0.254480206 & & \\
\hline 244140291 & & \\
\hline & & \\
\hline & & \\
\hline 248200928 & & \\
\hline 2584544 & & \\
\hline 260071046 & & \\
\hline .262671874 & & \\
\hline-0.2646902 & & \\
\hline-0.270984564 & 1603014 & 0.0 \\
\hline-0.272996573 & 9524283 & \\
\hline-0.27415141 & 1129 & \\
\hline-0.275841965 & 3996 & \\
\hline-0.276226719 & & \\
\hline & & \\
\hline-0.297651608 & & \\
\hline$-0.3027 C$ & & \\
\hline-0.302885456 & & 0.0 \\
\hline-0.306241068 & 11. & 0.0 \\
\hline-0.308280506 & 16.6 & 0.0 \\
\hline-0.31280088 & 15. & 0.0 \\
\hline-0.320028198 & 11. & 0.0 \\
\hline-0.324738732 & & \\
\hline & & \\
\hline & & \\
\hline & & \\
\hline-0.3336 & & \\
\hline & & 0.0 \\
\hline-0.338402188 & 6.3 & 0.0 \\
\hline-0.340262798 & 696 & 0.0 \\
\hline-0.34607507 & & \\
\hline 0202078 & & 0.0 \\
\hline & & \\
\hline & & \\
\hline & & \\
\hline & & \\
\hline & & 0.0 \\
\hline & & \\
\hline-0.36707 & 8.3 & $0.0 c$ \\
\hline-0.373063734 & 14. & 7822 \\
\hline-0.373125451 & 10. & 4552 \\
\hline-0.374930748 & 6.1 & 7743 \\
\hline-0.376113158 & 242 & 0.0 \\
\hline & 13.4 & \\
\hline & & \\
\hline & & \\
\hline & & \\
\hline & & \\
\hline-0.383331981 & 7.96 & 0.0 \\
\hline-0.38798432 & & \\
\hline-0.392 & 8.47 & 49524 \\
\hline-0.394704756 & 8.64 & 0.03 \\
\hline & 8.637270 & \\
\hline & 12.14565961 & \\
\hline & & \\
\hline
\end{tabular}


1

2

3

4

5

6

7

8

9

10

11

12

13

14

15

16

17

18

19

20

21

22

23

24

25

26

27

28

29

30

31

32

33

34

35

36

37

38

39

40

41

42

43

44

45

46

47

48

49

50

51

52

53

54

55

56

57

58

59

60

\begin{tabular}{|c|c|c|}
\hline & & \\
\hline & 338211044 & \\
\hline & .50763342 & \\
\hline & & \\
\hline .407062662 & 10.13514035 & \\
\hline & 7.689265574 & \\
\hline & & \\
\hline 0.411 & 72315 & \\
\hline & 2337 & \\
\hline 6155848 & 10.31468171 & \\
\hline 0019862 & 21489 & \\
\hline & 7948 & \\
\hline 47 & 6.602 & \\
\hline 3 & $8.52 \mathrm{c}$ & \\
\hline & 6.834 & \\
\hline 425 & 9.598 & \\
\hline & 12.20 & \\
\hline & & \\
\hline 06 & 13.84 & \\
\hline & & \\
\hline 69 & 6.700 & \\
\hline 68 & 7.142 & \\
\hline & & \\
\hline 78 & 6.892 & \\
\hline & 8.67 & \\
\hline & & \\
\hline 62 & 10. & \\
\hline & & \\
\hline & 8.9 & \\
\hline & & \\
\hline & & \\
\hline & 10. & \\
\hline & & \\
\hline & & \\
\hline & 966 & \\
\hline & & \\
\hline & 6.72 & \\
\hline & & \\
\hline & & \\
\hline & 3503 & \\
\hline & & \\
\hline-0.4 & & \\
\hline & 8.59 & \\
\hline & 052 & \\
\hline & & \\
\hline & & \\
\hline-0.4 & & \\
\hline & 2746 & \\
\hline & & \\
\hline & 7.7806 & \\
\hline & 7.624 & \\
\hline-0.47 & 69326 & \\
\hline & 12.83928633 & \\
\hline & & \\
\hline & 7.753841713 & 0.0069249 \\
\hline & 7.441660797 & \\
\hline-0.486825904 & 11.34420614 & \\
\hline & 11.17769638 & \\
\hline-0.487538832 & & \\
\hline
\end{tabular}

https://mc04.manuscriptcentral.com/cares 


\begin{tabular}{|c|c|c|}
\hline & & \\
\hline 93057428 & 10.15897212 & \\
\hline .495838288 & 8.738892753 & \\
\hline .495867287 & 10.35448148 & \\
\hline 6971605 & .68312203 & \\
\hline & & \\
\hline & & \\
\hline .502660256 & & \\
\hline & & \\
\hline & & \\
\hline 420231 & & \\
\hline 3979684 & & 0.00050924 \\
\hline 6346906 & 10.812 & \\
\hline 9338122 & 6.4567 & 7.1 \\
\hline 737911 & 6.3040 & \\
\hline 561067 & 9.9115 & \\
\hline 50808 & 16.61 & \\
\hline & & \\
\hline & & \\
\hline & & \\
\hline & & 2717 \\
\hline 552 & 12.0 & 0.0 \\
\hline 288 & 7.664 & 0.0 \\
\hline 824 & 11.477 & 0.01 \\
\hline 817 & & \\
\hline-0.5 & 6.85 & $0 .($ \\
\hline & & \\
\hline & & \\
\hline & & \\
\hline & & \\
\hline & 7.2 & 0. \\
\hline & & 0. \\
\hline 42 & 9.96 & 0.0 \\
\hline-0.53 & 8.61 & 379 \\
\hline & & 0 \\
\hline-0.5 & & \\
\hline & & \\
\hline & & \\
\hline & & \\
\hline & & \\
\hline & & 0.0 \\
\hline & & \\
\hline-0.5 & 7.770 & 0.0 \\
\hline-0.5 & 7.7923 & 7771 \\
\hline & 10.3 & 1260 \\
\hline-0.5 & 11.63 & 0.0 \\
\hline & & 0. \\
\hline & 7.19 & \\
\hline & & \\
\hline & & \\
\hline & & \\
\hline & & \\
\hline & & \\
\hline & & 0.0 \\
\hline & 9.2936 & 0.00 \\
\hline & & \\
\hline & & \\
\hline & & \\
\hline & & \\
\hline
\end{tabular}


1

2

3

4

5

6

7

8

9

10

11

12

13

14

15

16

17

18

19

20

21

22

23

24

25

26

27

28

29

30

31

32

33

34

34
35

36

37

38

39

40

41

42

43

44

45

46

47

48

49

50

51

52

53

54

55

56

57

58

59

60

\begin{tabular}{|c|c|c|}
\hline & 981610299 & \\
\hline 0.593172704 & 9.552982166 & \\
\hline 0.593215762 & 6.518906201 & \\
\hline & 6.01148154 & \\
\hline 774845 & .341019473 & \\
\hline & 12.43387167 & \\
\hline 0.599845312 & 8.583303218 & 0.02 \\
\hline 0.601224344 & 7.784270421 & 0.00 \\
\hline & 6031 & \\
\hline & 9.9388 & \\
\hline & $12.67 \mathrm{~g}$ & \\
\hline & 11.963 & \\
\hline & 8.9231 & \\
\hline & 8.43 & \\
\hline & 8.03 & \\
\hline & 8.64 & \\
\hline & 585 & \\
\hline 594 & 13.5 & \\
\hline & 10.3 & \\
\hline & & \\
\hline & 10.8 & \\
\hline & 7.84 & \\
\hline & 375 & \\
\hline & 6.8 & \\
\hline & & \\
\hline & 11.8 & \\
\hline & 10. & \\
\hline & & \\
\hline & 6.62 & \\
\hline & & \\
\hline & 213 & \\
\hline & 45 & \\
\hline & & \\
\hline & 6.80 & \\
\hline & & \\
\hline & & \\
\hline & & \\
\hline & & \\
\hline & 8.8 & \\
\hline & 8.32 & \\
\hline & & \\
\hline & & \\
\hline & 10.8 & \\
\hline & 9.64 & \\
\hline 881529 & 6.5812 & \\
\hline & 12.345 & \\
\hline & & \\
\hline & & \\
\hline & 8.0422 & \\
\hline 75928149 & & 0.00 \\
\hline & 8.3861 & \\
\hline & 10.802 & 0.01 \\
\hline & & \\
\hline & 6.703695122 & \\
\hline-0.682059235 & 7.962313068 & 0.0038787 \\
\hline & 10.13531086 & \\
\hline & 13.75316692 & \\
\hline & 11.21104891 & 0.0058841 \\
\hline-0.688651928 & 6812251 & 0.000481 \\
\hline
\end{tabular}

https://mc04.manuscriptcentral.com/cares 


\begin{tabular}{|c|c|c|}
\hline 3552 & & \\
\hline-0.698752868 & 12.3034 & 0. \\
\hline .70174101 & 03967 & 015 \\
\hline 701847616 & 10.4 & \\
\hline 9050858 & .22405549 & \\
\hline .709331688 & 7.351788502 & \\
\hline 0.710643542 & 12.49347357 & \\
\hline-0.71194352 & 7.574959028 & \\
\hline 3805022 & 9.633 & \\
\hline 21248 & & \\
\hline & & \\
\hline & & \\
\hline & & \\
\hline-0.7 & 669 & \\
\hline-0.72 & 015 & \\
\hline-0.7 & & \\
\hline 613 & 604 & \\
\hline 179 & & \\
\hline 216 & & \\
\hline & & \\
\hline & & \\
\hline & & \\
\hline & & \\
\hline & & \\
\hline-0.76 & & \\
\hline-0.76 & 9.7 & 0.0 \\
\hline-0.77 & 6.9 & 0.0 \\
\hline-0.77 & 9.1 & \\
\hline-0.7 & & \\
\hline & & \\
\hline & & \\
\hline & & \\
\hline & & \\
\hline & & \\
\hline-0.7 & & \\
\hline-0.75 & & \\
\hline-0.80 & & \\
\hline-0.8 & 8.3 & 0.0 \\
\hline & & \\
\hline & & \\
\hline-0.80 & & \\
\hline & & \\
\hline & & \\
\hline & & \\
\hline & & \\
\hline & & \\
\hline-0.8 & 12.7 & 0.0 \\
\hline-0.8 & 7.395 & 9.70 \\
\hline & & \\
\hline-0.8 & 8.20 & \\
\hline-0.84 & & 0.0 \\
\hline-0.84 & 10.3 & 0.0 \\
\hline-0.8 & & 0.00 \\
\hline & & \\
\hline & & \\
\hline & & $0.0027746 \varepsilon$ \\
\hline & & 1.27463E-C \\
\hline & & \\
\hline & & 0.0206085 \\
\hline
\end{tabular}


1

2

3

4

5

6

7

8

9

10

11

12

13

14

15

16

17

18

19

20

21

22

23

24

25

26

27

28

29

30

31

32

33

34

35

36

37

38

39

40

41

42

43

44

45

46

47

48

49

50

51

52

53

54

55

56

57

58

59

60

\begin{tabular}{|c|c|c|}
\hline & .71026659 & \\
\hline & & \\
\hline 6352 & 6173 & \\
\hline & & \\
\hline & 7.824256532 & \\
\hline-0.895878116 & 10.1105168 & \\
\hline 92384 & 6.8840 & \\
\hline & 10.5 & \\
\hline-0.90 & 9.18 & \\
\hline 030723 & 7.239072622 & \\
\hline 29989 & 10.02342185 & \\
\hline & 10.9 & \\
\hline-0.9 & 8.60 & \\
\hline 45 & 8.24 & \\
\hline 28 & 12.9 & \\
\hline & 6.87 & \\
\hline 28 & & \\
\hline & 6.69 & \\
\hline 26 & 10.5 & \\
\hline 76 & 10.3 & \\
\hline 12 & 7.04 & \\
\hline 49 & & \\
\hline & 12.7 & \\
\hline 73 & 8.02 & \\
\hline & 8.63 & \\
\hline 16 & 12.1 & \\
\hline-0.9 & 12.3 & \\
\hline & & \\
\hline & 9.1 & \\
\hline 08 & 12. & \\
\hline & & \\
\hline-0.9 & 8.0 & \\
\hline & 10. & \\
\hline & & \\
\hline & 36 & \\
\hline & & \\
\hline & 8.0 & \\
\hline & 7.2 & \\
\hline & 8.6 & \\
\hline 528 & 8.42 & \\
\hline & & \\
\hline & 56 & \\
\hline & & \\
\hline & & \\
\hline & 271 & \\
\hline & & \\
\hline & 6.8 & \\
\hline & 7.5 & \\
\hline & 9.36 & \\
\hline & 7.702 & \\
\hline & 7.24 & \\
\hline & 10.5 & \\
\hline & 7.487 & \\
\hline & 13.18253392 & \\
\hline & 7.714238037 & $9.10813 \mathrm{E}-0$ \\
\hline & 9.614315788 & \\
\hline & 7.882077667 & 0.0 \\
\hline-1.074317003 & 7.136480655 & 0.02 \\
\hline-1.083238495 & 8716056750 & \\
\hline
\end{tabular}

https://mc04.manuscriptcentral.com/cares 


\begin{tabular}{|c|c|c|}
\hline & & \\
\hline .088687615 & 10.28319672 & \\
\hline .089176143. & 9.227035955 & \\
\hline .093040424 & 627188001 & \\
\hline 3432417 & 365545407 & \\
\hline & & \\
\hline 097432887 & & \\
\hline-1.10377111 & 8.828398051 & \\
\hline & & \\
\hline 11 & $9.549 \varepsilon$ & \\
\hline 162 & & \\
\hline 4640221 & & \\
\hline 1.161813793 & 7.39053 & \\
\hline$-1.1723 c$ & 10.08 & \\
\hline-1.175193414 & 6044 & 2.3315 \\
\hline 176209151 & 8.320 & 0.028 \\
\hline & & \\
\hline & & \\
\hline & & \\
\hline & & \\
\hline 1.20 & 12.0 & 2.82 \\
\hline 08 & 12.7 & 8.8 \\
\hline 147 & 7.273 & 0.000 \\
\hline 6097007 & 8.675 & 2.227 \\
\hline-1.21 & 10.06 & 1.12 \\
\hline 17 & 9.95 & \\
\hline & & \\
\hline & & \\
\hline & & \\
\hline & & \\
\hline & & 8.6 \\
\hline & 7.4 & 0.0 \\
\hline-1.27 & 7.69 & 1.2 \\
\hline-1.27 & 11.4 & 0.00 \\
\hline & 12 & 9.4 \\
\hline & & \\
\hline & & \\
\hline & & \\
\hline & & \\
\hline & & \\
\hline & & 1.23 \\
\hline & & \\
\hline & 6.98 & 2.9 \\
\hline 65 & 6.75 & 0.00 \\
\hline-1.3 & 10.74 & 4.63 \\
\hline & 13.1 & 8.90 \\
\hline & 11.3 & 4.60 \\
\hline & 8.010 & \\
\hline & $9.4 \varsigma$ & 0.02 \\
\hline & & \\
\hline & & \\
\hline & & \\
\hline & & \\
\hline & & \\
\hline & 10.16522609 & $1.02537 \mathrm{E}-\mathrm{C}$ \\
\hline & 9.054592244 & 0.00012463 \\
\hline & & \\
\hline & & 0.01144772 \\
\hline & & \\
\hline
\end{tabular}


1

2

3

4

5

6

7

8

9

10

11

12

13

14

15

16

17

18

19

20

21

22

23

24

25

26

27

28

29

30

31

32

33

34

35

36

37

38

39

40

41

42

43

44

45

46

47

48

49

50

51

52

53

54

55

56

57

58

59

60

\begin{tabular}{|c|c|c|}
\hline-1.417803996 & 10.06687358 & \\
\hline-1.418944898 & 9.618498483 & \\
\hline-1.431784462 & 9.098136864 & \\
\hline & 7.7543 & \\
\hline-1.46036708 & 14.50307495 & \\
\hline-1.476230322 & 7.936182419 & \\
\hline-1.485576479 & 8.419571885 & \\
\hline-1.491097654 & 8.464035327 & \\
\hline 034 & 792 & \\
\hline 6524 & 11.03368948 & \\
\hline 07736 & 10.44255926 & \\
\hline 3841 & 27945 & \\
\hline-1.562962584 & 11.8 & \\
\hline-1.5 & 919 & \\
\hline 5677 & 70501 & \\
\hline 8222 & 77656 & \\
\hline-1.60 & & \\
\hline-1.62 & & \\
\hline & 7.04 & \\
\hline 3464 & & \\
\hline 0724 & 0198 & \\
\hline-1.6 & 9.22 & \\
\hline & & \\
\hline-1.7 & 263 & \\
\hline-1.7 & 10. & \\
\hline-1.72 & 9.34 & \\
\hline-1.7 & 8.7 & \\
\hline & & \\
\hline-1.8 & 12. & \\
\hline-1.8 & & \\
\hline & 3681 & \\
\hline$-1 . c$ & 8.5 & \\
\hline & 13. & \\
\hline-2.0 & 9.27 & 0.0 \\
\hline-2.02 & 9.154942083 & \\
\hline & 11.7 & \\
\hline-2.2 & & \\
\hline$-2.2 \varepsilon$ & 13 & \\
\hline-2.32 & 48441 & \\
\hline-2.566482682 & 9.918835431 & 1.5 \\
\hline-2.708851464 & 7.72208074 & \\
\hline-2.8 & 8.0 & \\
\hline-2.990439512 & 11.37800517 & \\
\hline-3.085193903 & 8.567395379 & \\
\hline-3.096400623 & 9.286752712 & 4.62 \\
\hline-3.146347755 & & \\
\hline-3.150307147 & 11.50210939 & 8.73791E-1 \\
\hline-3.218676372 & 12.76378133 & $9.75281 \mathrm{E}-1$ \\
\hline-3.381084819 & 9.712777677 & $3.4835 \mathrm{E}-1$ \\
\hline-3.517707117 & 8.237049671 & 0.001114 \\
\hline-4.328433054 & 11.49218277 & \\
\hline-6.156879098 & 9.98739195 & $1.65288 \mathrm{E}$ \\
\hline
\end{tabular}


Supplimentary Table 2. List of genes validated by real-time PCR.

\begin{tabular}{|c|c|c|c|c|c|c|}
\hline Gene Symbol & Gene Name & FC uArr & Ave Expr & Adj.p.value & FC pcr & p.value \\
\hline Trim12a & tripartite motif-containing $12 \mathrm{~A}$ & 40.13 & 7.97 & $1.5 \mathrm{E}-21$ & 20.31 & 0.0002 \\
\hline $\mathrm{Ccl} 27 \mathrm{a}$ & $\begin{array}{l}\text { chemokine (C-C motif) ligand } \\
27 \mathrm{~A}\end{array}$ & 8.94 & 8.53 & $2.5 \mathrm{E}-15$ & 4.30 & 0.0107 \\
\hline $\begin{array}{l}\text { Ccl27a } \\
\text { (Pesky) }\end{array}$ & $\begin{array}{l}\text { chemokine (C-C motif) ligand } \\
\text { 27A Pesky }\end{array}$ & 2.83 & 10.15 & $1.1 \mathrm{E}-03$ & 4.30 & 0.0001 \\
\hline Car9 & carbonic anhydrase 9 & 4.68 & 11.43 & $1.1 \mathrm{E}-02$ & 3.83 & 0.0119 \\
\hline Ptchd2 & patched domain containing 2 & 4.00 & 7.33 & $1.7 \mathrm{E}-13$ & 7.33 & 0.0000 \\
\hline Slc2a4 & $\begin{array}{l}\text { solute carrier family } 2 \text { (facilitated } \\
\text { glucose transporter), member } 4\end{array}$ & 3.91 & 8.57 & 8.5E-09 & 5.25 & 0.0009 \\
\hline Bmp4 & bone morphogenetic protein 4 & 3.31 & 12.83 & $2.0 \mathrm{E}-03$ & 3.11 & 0.0002 \\
\hline Kcnab1 & $\begin{array}{l}\text { potassium voltage-gated channel, } \\
\text { shaker-related subfamily, beta } \\
\text { member } 1\end{array}$ & 3.14 & 9.03 & $2.5 \mathrm{E}-04$ & 1.72 & 0.0223 \\
\hline P2ry2 & $\begin{array}{l}\text { purinergic receptor P2Y, G-protein } \\
\text { coupled } 2\end{array}$ & 2.29 & 10.79 & $4.0 \mathrm{E}-04$ & 3.45 & 0.0000 \\
\hline Tnrc18 & trinucleotide repeat containing 18 & 1.70 & 11.77 & $2.5 \mathrm{E}-03$ & 1.79 & 0.0126 \\
\hline TGFb3 & transforming growth factor, beta 3 & 1.46 & 14.82 & $4.5 \mathrm{E}-02$ & 1.80 & 0.0122 \\
\hline Vcp & valosin containing protein & 1.37 & 15.31 & $9.0 \mathrm{E}-04$ & 1.50 & 0.0012 \\
\hline Ankib1 & $\begin{array}{l}\text { ankyrin repeat and IBR domain } \\
\text { containing } 1\end{array}$ & 1.28 & 11.36 & 2.7E-02 & 1.41 & 0.0115 \\
\hline Panx1 & pannexin 1 & -1.8657 & 10.51056 & $2.4 \mathrm{E}-02$ & -4.48 & 0.032 \\
\hline Cacna2d3 & $\begin{array}{l}\text { calcium channel, voltage- } \\
\text { dependent, alpha } 2 / \text { delta subunit } 3\end{array}$ & -1.94831 & 8.02254 & $2.1 \mathrm{E}-02$ & -1.90 & 0.040 \\
\hline Fbln2 & fibulin 2 & -2.13 & 12.94 & $3.5 \mathrm{E}-02$ & -2.17 & 0.016 \\
\hline Grm5 & glutamate receptor, metabotropic 5 & -2.72 & 7.75 & $3.0 \mathrm{E}-02$ & -4.64 & 0.023 \\
\hline Chl1 & $\begin{array}{l}\text { cell adhesion molecule with } \\
\text { homology to L1CAM }\end{array}$ & -2.90 & 10.44 & $1.7 \mathrm{E}-03$ & -2.13 & 0.0410 \\
\hline Col2a1 & collagen, type II, alpha 1 & -3.03 & 10.26 & $3.0 \mathrm{E}-02$ & -2.83 & 0.068 \\
\hline Gap43 & growth associated protein 43 & -3.05 & 10.06 & $2.3 \mathrm{E}-03$ & -3.03 & 0.0497 \\
\hline Lynx1 & Ly6/neurotoxin 1 & -3.29 & 11.50 & $8.9 \mathrm{E}-07$ & -4.62 & 0.0050 \\
\hline Lpl & lipoprotein lipase & -3.89 & 10.54 & $2.1 \mathrm{E}-03$ & -5.57 & 0.0141 \\
\hline Padi2 & $\begin{array}{l}\text { peptidyl arginine deiminase, type } \\
\text { II }\end{array}$ & -3.94 & 13.12 & $2.9 \mathrm{E}-06$ & -2.54 & 0.025 \\
\hline $\mathrm{P} 2 \mathrm{rx} 7$ & $\begin{array}{l}\text { purinergic receptor P2X, ligand- } \\
\text { gated ion channel, } 7\end{array}$ & -5.92 & 9.92 & $1.6 \mathrm{E}-05$ & -2.45 & 0.0004 \\
\hline Pcdh17 & protocadherin 17 & -8.55 & 9.29 & $4.6 \mathrm{E}-09$ & -3.64 & 0.0058 \\
\hline Samd4 & $\begin{array}{l}\text { sterile alpha motif domain } \\
\text { containing } 4\end{array}$ & -10.42 & 9.71 & $3.5 \mathrm{E}-12$ & -24.32 & 0.0000 \\
\hline Col4a6 & collagen, type IV, alpha 6 & -20.09 & 11.49 & $1.7 \mathrm{E}-16$ & -24.89 & 0.0003 \\
\hline
\end{tabular}




\section{Ranked list of genes up-regulated in non-Tg compared to ac AGILENT SurePrint G3 GE 8x60k probc AFFY Mouse430 2 probe}

\begin{tabular}{|c|c|}
\hline A_52_P244193 & 1437502_x_at \\
\hline A_55_P2068673 & 1423281_at \\
\hline A_52_P244193 & 1448182_a_at \\
\hline A_55_P2068673 & 1423280_at \\
\hline A_55_P1959748 & 1433966_x_at \\
\hline A_55_P2144126 & 1437185_s_at \\
\hline A_52_P504268 & 1418736_at \\
\hline A_55_P2022569 & 1435486_at \\
\hline A_51_P404463 & 1460049_s_at \\
\hline A_52_P105537 & 1426851_a_at \\
\hline A_52_P244193 & 1416034_at \\
\hline A_55_P1995205 & 1454694_a_at \\
\hline A_52_P360330 & 1423274_at \\
\hline A_55_P2096867 & 1423537_at \\
\hline A_55_P1964638 & 1453282_at \\
\hline A_51_P126437 & 1420965_a_at \\
\hline A_52_P220440 & 1426712_at \\
\hline A_52_P93837 & 1455961_at \\
\hline A_55_P2092085 & 1436902_x_at \\
\hline A_55_P2033362 & 1427682_a_at \\
\hline A_55_P2081040 & 1434848_at \\
\hline A_52_P229052 & 1441598_at \\
\hline A_52_P285024 & 1454877_at \\
\hline A_52_P257812 & 1415904_at \\
\hline A_55_P2016647 & 1448314_at \\
\hline A_55_P1955457 & 1425458_a_at \\
\hline A_52_P470466 & 1434685_at \\
\hline A_52_P24308 & 1448260_at \\
\hline A_55_P2085142 & 1449254_at \\
\hline A_51_P366061 & 1416514_a_at \\
\hline A_52_P105537 & 1426852_x_at \\
\hline A_55_P2056325 & 1460330_at \\
\hline A_51_P455997 & 1452905_at \\
\hline A_55_P2031021 & 1448595_a_at \\
\hline A_52_P569023 & 1454926_at \\
\hline A_66_P101600 & 1424113_at \\
\hline A_55_P1954221 & 1416529_at \\
\hline A_52_P667287 & 1434418_at \\
\hline A_55_P2100475 & 1455980_a_at \\
\hline A_55_P1991016 & 1428572_at \\
\hline A_51_P455997 & 1439380_x_at \\
\hline A_51_P404463 & 1423261_at \\
\hline A_51_P394997 & 1418047_at \\
\hline A_55_P2065424 & 1422256_at \\
\hline
\end{tabular}


A_51_P200561

A_55_P2032412

A_52_P203316

A_51_P112734

A_52_P502577

A_55_P2151388

A_55_P2011436

A_55_P1966204

A_51_P405606

A_55_P2215880

A_51_P100208

A_55_P2017418

A_52_P87839

A_51_P295085

A_55_P1959748

A_55_P2006629

A_51_P484111

A_51_P163953

A_55_P2000521

A_55_P2003813

A_52_P462472

A_51_P107362

A_55_P2042486

A_55_P2006629

A_51_P278653

A_55_P2052062

A_52_P599317

A_51_P353252

A_51_P230098

A_55_P2026530

A_55_P2074736

A_52_P568235

A_52_P628915

A_55_P2031836

A_55_P2041828

A_52_P331727

A_55_P2110512

A_55_P2031836

A_55_P2007155

A_52_P162486

A_51_P105709

A_55_P2017636

A_51_P254855

A_52_P49042

A_55_P2025033

A_51_P126437

A_55_P2031836

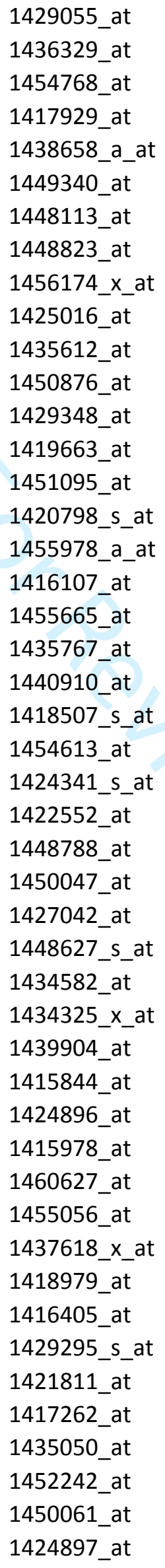




\begin{tabular}{|c|c|}
\hline A_55_P2085060 & 1436094_at \\
\hline A_52_P263518 & 1428156_at \\
\hline A_51_P127738 & 1435933_at \\
\hline A_55_P2111302 & 1448735_at \\
\hline A_55_P1980631 & 1451246_s_at \\
\hline A_52_P351925 & 1452124_at \\
\hline A_51_P317031 & 1418778_at \\
\hline A_55_P2024525 & 1455925_at \\
\hline A_55_P2084646 & 1455516_at \\
\hline A_55_P2158251 & 1440177_at \\
\hline A_51_P465128 & 1452092_at \\
\hline A_52_P925277 & 1435227_at \\
\hline A_55_P1964638 & 1427321_s_at \\
\hline A_51_P405606 & 1420760_s_at \\
\hline A_51_P494293 & 1453245_at \\
\hline A_51_P247157 & 1438988_x_at \\
\hline A_51_P389156 & 1420994_at \\
\hline A_52_P413646 & 1450759_at \\
\hline A_55_P2063462 & 1449286_at \\
\hline A_55_P2412847 & 1426016_a_at \\
\hline A_55_P2100555 & 1450435_at \\
\hline A_51_P366207 & 1418046_at \\
\hline A_66_P114461 & 1443119_at \\
\hline A_55_P2128511 & 1435917_at \\
\hline A_51_P398037 & 1439204_at \\
\hline A_55_P2380970 & 1448083_at \\
\hline A_51_P360492 & 1438852_x_at \\
\hline A_55_P2013336 & 1416558_at \\
\hline A_55_P2033362 & 1427683_at \\
\hline A_51_P289889 & 1452107_s_at \\
\hline A_55_P2003216 & 1460214_at \\
\hline A_52_P263518 & 1428157_at \\
\hline A_51_P427663 & 1450981_at \\
\hline A_55_P2102385 & 1426880_at \\
\hline A_52_P470466 & 1427247_at \\
\hline A_55_P2044587 & 1436662_at \\
\hline A_52_P21 & 1436237_at \\
\hline A_51_P455997 & 1452183_a_at \\
\hline A_55_P2082653 & 1432750_at \\
\hline
\end{tabular}




\section{:utely isolated astrocytes}

\section{AGILENT Gene Symbol}

Cd24a

Stmn2

Cd24a

Stmn2

Asns

B3galnt1

Pak3

1500015010Rik

Nov

Cd24a

Top2a

Map1b

Gap43

Cxadr

Enc1

Slc6a15

Mme

Tmsb10

Egr2

Gpr27

Tmeff2

Sertad4

Lpl

Cdk1

Grb10

D3Bwg0562e

Uchl1

Spp1

Fscn1

Nov

Anxa3

Meg3

Bex1

Sphkap

Lamb1

Emp1

Cers6

Gas213

Basp1

Meg3

1500015010Rik

Neurod6

Sstr2

\section{AFFY Gene Symbol}

$\mathrm{Cd} 24 \mathrm{a}$

Stmn2

$\mathrm{Cd} 24 \mathrm{a}$

Stmn2

Asns

0 Tmsb10

B3galnt1

Pak3

1500015010Rik

Nov

Cd24a

Top2a

Ints6

Gap43

Cxadr

Enc1

Slc6a15

Mme

Tmsb10

Egr2

Tmeff2

Sertad4

Lpl

Cdk1

Grb10

D3Bwg0562e

Uchl1

Spp1

Fscn1

Nov

Anxa3

Meg3

Bex1

Sphkap

Lamb1

Emp1

Cers6

Gas2l3

Basp1

Meg3

1500015010Rik

Neurod6

Sstr2 q-value

0
0
0
0
0
0
0
0
0
0
0
0
0

2.38E-04

2.38E-04

2.38E-04

2.38E-04

2.38E-04

2.38E-04

2.38E-04

2.38E-04

2.38E-04

2.38E-04

2.38E-04

2.38E-04

2.38E-04

2.38E-04

2.38E-04

2.38E-04

2.38E-04

2.38E-04

2.08E-04

3.03E-04

3.92E-04

3.92E-04

3.92E-04

3.92E-04

6.14E-04

7.69E-04

8.33E-04

8.94E-04

8.94E-04

8.94E-04

9.09E-04 


\begin{tabular}{|c|c|c|}
\hline 4930506M07Rik & 4930506M07Rik & $9.09 \mathrm{E}-04$ \\
\hline Egr3 & Egr3 & $9.09 \mathrm{E}-04$ \\
\hline Kenf1 & Kcnf1 & $9.22 \mathrm{E}-04$ \\
\hline Slc7a8 & Slc7a8 & $9.72 \mathrm{E}-04$ \\
\hline S1pr3 & S1pr3 & $9.72 \mathrm{E}-04$ \\
\hline Sostdc1 & Sostdc1 & $9.72 \mathrm{E}-04$ \\
\hline Gm11223 & Stmn1 & 0.001045752 \\
\hline Cxcl12 & Cxcl12 & 0.001045752 \\
\hline Ndrg1 & Ndrg1 & 0.001069182 \\
\hline Ephb2 & Ephb2 & 0.00117284 \\
\hline Opcml & Opcml & 0.001212121 \\
\hline Cfh & Cfh & 0.001212121 \\
\hline Sema3c & Sema3c & 0.001212121 \\
\hline Ogn & Ogn & 0.001206897 \\
\hline \multirow[t]{2}{*}{ Asns } & Asns & 0.001206897 \\
\hline & 0 Pcdha1 | Pcdha10 | Pcdha11 | & 0.001206897 \\
\hline Matn2 & Matn2 & 0.001206897 \\
\hline Nsg2 & Nsg2 & 0.001290323 \\
\hline Lonrf1 & Lonrf1 & 0.001640212 \\
\hline Scn3b & Scn3b & 0.001666667 \\
\hline C77370 & C77370 & 0.001666667 \\
\hline Socs2 & Socs2 & 0.001666667 \\
\hline \multirow[t]{2}{*}{ Dpysl3 } & Dpysl3 & 0.001666667 \\
\hline & 0 Pcdha1 | Pcdha10 | Pcdha11 | & 0.001666667 \\
\hline Rprm & Rprm & 0.001642512 \\
\hline $\mathrm{Cd} 200$ & $\mathrm{Cd} 200$ & 0.001642512 \\
\hline Hs6st2 & Hs6st2 & 0.001642512 \\
\hline Mal2 & Mal2 & 0.00162037 \\
\hline Pbk & Pbk & 0.00162037 \\
\hline Erc2 & Erc2 & 0.001621622 \\
\hline Prkar1b & Prkar1b & 0.002044444 \\
\hline Fstl5 & Fstl5 & 0.002061404 \\
\hline Syt4 & Syt4 & 0.002061404 \\
\hline Gpr85 & Gpr85 & 0.002094017 \\
\hline Tubb3 & Tubb3 & 0.002109705 \\
\hline Thsd7b & Thsd7b & 0.002333333 \\
\hline Lmo7 & Lmo7 & 0.002333333 \\
\hline Gpr85 & Gpr85 & 0.002333333 \\
\hline Akr1c14 & Akr1c14 & 0.002329317 \\
\hline Bgn & Bgn & 0.002329317 \\
\hline Trip13 & Trip13 & 0.002329317 \\
\hline Thbs1 & Pald1 | Thbs1 & 0.00248062 \\
\hline Ptgs2 & Ptgs2 & 0.00248062 \\
\hline D10Bwg1379e & D10Bwg1379e & 0.00248062 \\
\hline Cep55 & Cep55 & 0.002509363 \\
\hline Enc1 & Enc1 & 0.002555556 \\
\hline Gpr85 & Gpr85 & 0.002857143 \\
\hline
\end{tabular}




\begin{tabular}{|c|c|c|}
\hline Vgf & Vgf & 0.002857143 \\
\hline Gng2 & Gng2 & 0.003046595 \\
\hline Scn2a1 & Scn2a1 & 0.003046595 \\
\hline $\mathrm{Cp}$ & $\mathrm{Cp}$ & 0.003017544 \\
\hline Aurkb & Aurkb & 0.003125 \\
\hline Ank3 & Ank3 & 0.003127148 \\
\hline Ccdc109b & Ccdc109b & 0.003127148 \\
\hline Prdm8 & Prdm8 & 0.003127148 \\
\hline Csrnp3 & Csrnp3 & 0.003266667 \\
\hline Grik3 & Grik3 & 0.003432343 \\
\hline Chst15 & Chst15 & 0.00372549 \\
\hline $\mathrm{Bcl} 11 \mathrm{~b}$ & Bcl11b & 0.003883495 \\
\hline Cxadr & Cxadr & 0.003942308 \\
\hline Ndrg1 & Ndrg1 & 0.003942308 \\
\hline 9130024F11Rik & 9130024F11Rik & 0.004025157 \\
\hline $\mathrm{Hn} 1$ & $\mathrm{Hn} 1$ & 0.004049844 \\
\hline B3gnt5 & B3gnt5 & 0.004290124 \\
\hline \multirow[t]{2}{*}{ Bmp6 } & Bmp6 & 0.00440367 \\
\hline & 0 Ntng1 & 0.00440367 \\
\hline Tro & Tro & 0.004384385 \\
\hline L1cam & L1cam & 0.004375 \\
\hline Nap1/2 & Nap1/2 & 0.004483776 \\
\hline Grm7 & Grm7 & 0.004473684 \\
\hline Ociad2 & Ociad2 & 0.004550725 \\
\hline Scn3a & $\operatorname{Scn} 3 a$ & 0.00454023 \\
\hline Nalcn & Nalcn & 0.004586895 \\
\hline Mcm6 & Mcm6 & 0.004661017 \\
\hline Melk & Melk & 0.00464986 \\
\hline Egr2 & Egr2 & 0.004833333 \\
\hline Npnt & Npnt & 0.004903582 \\
\hline Igsf5 & Pcp4 & 0.004903582 \\
\hline Gng2 & Gng2 & 0.004850949 \\
\hline Cnn2 & Cnn2 & 0.004850949 \\
\hline Et|4 & Et/4 & 0.0048 \\
\hline D3Bwg0562e & D3Bwg0562e & 0.00489418 \\
\hline Sorcs1 & Sorcs1 & 0.00488189 \\
\hline Ttc9 & Ttc9 & 0.00488189 \\
\hline Meg3 & Meg3 & 0.004909561 \\
\hline Zfp711 & Zfp711 & 0.004948718 \\
\hline
\end{tabular}




\section{RP-value}

34.223267

39.382553

41.796303

58.87709

78.59536

118.15399

130.00249

130.11092

142.4315

145.698

147.38728

153.62456

155.22014

164.82515

168.33496

169.44853

170.17828

173.7218

175.71043

178.14516

179.20235

181.67484

183.20612

191.8282

193.05821

195.35397

196.87836

196.96948

200.75273

201.90858

202.42519

205.22661

210.77614

218.74706

220.83049

223.22322

225.10469

235.67517

244.70367

256.50943

263.50482

263.89993

263.93997

275.0427 


$$
\begin{aligned}
& 275.65512 \\
& 276.24673 \\
& 277.1194 \\
& 277.63657 \\
& 279.0203 \\
& 279.34738 \\
& 281.23773 \\
& 282.6501 \\
& 284.16025 \\
& 287.8079 \\
& 290.9738 \\
& 291.76346 \\
& 293.47815 \\
& 294.5826 \\
& 296.6824 \\
& 296.96982 \\
& 298.0942 \\
& 306.06696 \\
& 319.91132 \\
& 324.035 \\
& 325.75204 \\
& 327.43738 \\
& 327.51932 \\
& 327.8075 \\
& 329.64703 \\
& 331.24893 \\
& 336.4755 \\
& 338.52478 \\
& 340.3146 \\
& 341.4727 \\
& 349.87045 \\
& 351.45538 \\
& 355.1068 \\
& 358.2844 \\
& 359.173 \\
& 366.26736 \\
& 367.05988 \\
& 371.30234 \\
& 378.1268 \\
& 378.5343 \\
& 378.63394 \\
& 382.84604 \\
& 383.13834 \\
& 383.91983 \\
& 385.2477 \\
& 386.46875 \\
& 399.72336
\end{aligned}
$$




$$
\begin{array}{r}
399.76904 \\
407.29984 \\
407.35904 \\
409.69595 \\
412.1374 \\
413.18622 \\
413.26825 \\
413.3775 \\
423.0475 \\
426.3158 \\
432.8777 \\
438.29535 \\
439.4035 \\
439.8584 \\
444.10327 \\
445.91617 \\
450.83182 \\
455.31924 \\
456.33588 \\
457.64203 \\
458.6556 \\
461.70532 \\
462.412 \\
463.3562 \\
464.0969 \\
464.66165 \\
465.9082 \\
467.39966 \\
470.19315 \\
472.44604 \\
472.52155 \\
473.28952 \\
473.4576 \\
474.54385 \\
476.9111 \\
477.72067 \\
478.11398 \\
480.9673 \\
482.90833
\end{array}
$$




\section{Ranked list of genes down-regulated in non-Tg compared tc} AGILENT SurePrint G3 GE 8x60k probı AFFY Mouse430 2 probe

A_52_P482251

A_51_P208603

A_51_P155873

A_55_P1970090

A_51_P391616

A_51_P406557

A_51_P468260

A_55_P2045299

A_55_P1993503

A_52_P67212

A_51_P355427

A_51_P136303

A_51_P452768

A_52_P266686

A_51_P430929

A_51_P391616

A_51_P268193

A_55_P1955412

A_55_P2427900

A_51_P127297

A_55_P1971599

A_51_P207988

A_55_P2013701

A_51_P468260

A_55_P1955627

A_55_P2003266

A_51_P431543

A_51_P443819

A_55_P1955627

A_55_P1961913

A_55_P2054673

A_51_P321341

A_55_P2162712

A_52_P177847

A_51_P285077

A_66_P109397

A_55_P2033312

A_52_P177847

A_55_P2002849

A_66_P109368

A_51_P504354

A_55_P2123484

A_55_P2007447

A_51_P140641 1448397_at

1418937_at

1428923_at

1449245_at

1441430_at

1435417_at

1419383_at

1457536_at

1434430_s_at

1457495_at

1423405_at

1449316_at

1419559_at

1417151_a_at

1433653_at

1452975_at

1421093_at

1451627_a_at

1455304_at

1449038_at

1416718_at

1424208_at

1427284_a_at

1434342_at

1424945_at

1435918_at

1429647_at

1425408_a_at

1456722_at

1448602_at

1418050_at

1427345_a_at

1435314_at

1428922_at

1424553_at

1457373_at

1434121_at

1452961_at

1434742_s_at

1436569_at

1417985_at

1442917_at

1417946_at

1424652_at 
A_55_P2003266

A_52_P532227

A_55_P2056483

A_52_P622850

A_55_P2156304

A_55_P2391065

A_55_P2036392

A_51_P124126

A_51_P375558

A_55_P2056483

A_51_P396883

A_51_P216702

A_51_P156857

A_55_P1955437

A_55_P2108599

A_55_P2070992

A_55_P2391065

A_55_P2029721

A_55_P2003266

A_55_P2108599

A_52_P85174

A_66_P139666

A_66_P114784

A_55_P1963508

A_55_P2036392

A_51_P169516

A_52_P883557

A_66_P124091

A_66_P105046

A_55_P2105472

A_66_P119457

A_55_P2027900

A_51_P396883

A_55_P2103225

A_52_P141687

A_51_P268697

A_51_P207988

A_51_P302566

A_52_P496956

A_51_P404077

A_51_P334942

A_51_P392005

A_55_P2034067

A_55_P1955412

A_66_P139666

A_51_P312336

A_55_P2002849

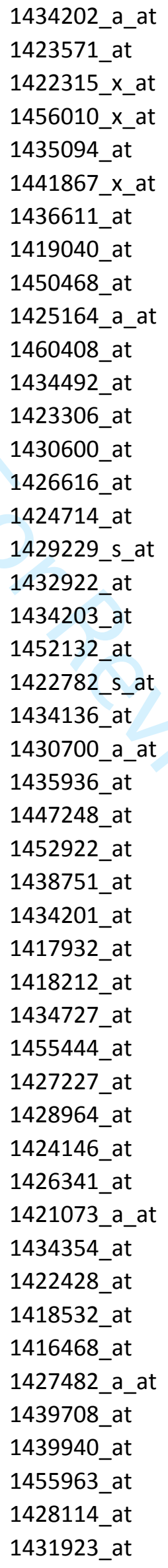


A_52_P376169

A_52_P368306

A_55_P1973033

A_51_P330044

A_52_P681391

A_51_P216702

A_51_P404077

A_52_P401504

A_55_P2396370

A_55_P2025228

A_55_P2388118

A_51_P268697

A_55_P2427900

A_55_P1961014

A_51_P276235

A_55_P2002572

A_55_P1964363

A_55_P1996674

A_55_P1972192

A_55_P1996578

A_55_P1972192

A_55_P2080021

A_55_P1964960

A_51_P510764

A_52_P655687

A_51_P209327

A_55_P1971889

A_55_P1972981

A_55_P2030373

A_51_P479230

A_51_P117477

A_55_P2096043

A_55_P2000369

A_55_P2018666

A_55_P2156304

A_55_P2133007

A_55_P1960416

A_30_P01022245

A_55_P2009752

A_66_P101835

A_55_P1952638

A_52_P457567

A_51_P496309

A_52_P376169

A_52_P174915

A_55_P2051885

A_52_P382886

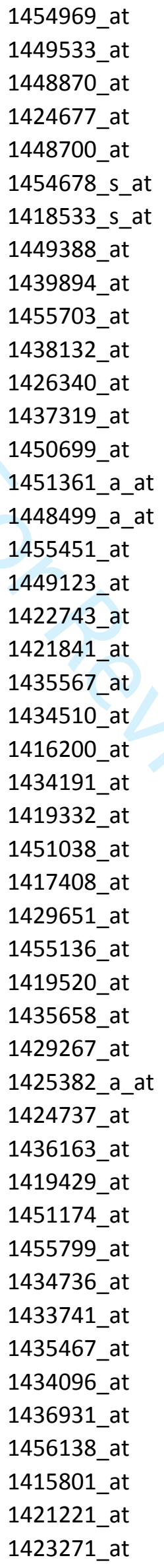


A_52_P309166

A_30_P01017882

A_55_P2061620

A_51_P204715

A_55_P1959923

A_55_P1972192

A_55_P1982454

A_52_P116264

A_55_P1961014

A_55_P2000369

A_55_P2024669

A_52_P309166

A_52_P622850

A_55_P2112270

A_55_P2062737

A_55_P1964363

A_51_P502150

A_55_P2055854

A_66_P112684

A_51_P125842

A_52_P586944

A_51_P426739

A_51_P258690

A_52_P571371

A_51_P131216

A_55_P2049752

A_66_P119457

A_55_P1996578

A_55_P1965616

A_52_P175376

A_51_P242201

A_55_P2078670

A_51_P216702

A_55_P2106901

A_52_P520495

A_55_P2168823

A_52_P62121

A_55_P2091858

A_51_P488991

A_51_P337523

A_51_P455647

A_51_P173709

A_52_P465886

A_55_P2009752

A_55_P2000148

A_51_P309854

A_52_P488427

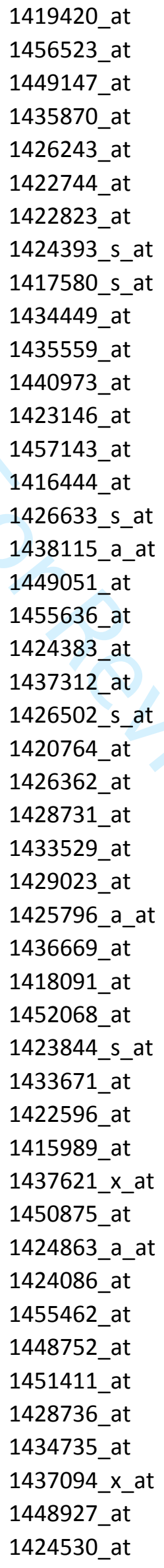




\begin{tabular}{|c|c|}
\hline A_51_P211793 & 1418310_a_at \\
\hline A_55_P1964363 & 1455452_x_at \\
\hline A_55_P2423586 & 1457424_at \\
\hline A_51_P242201 & 1452067_at \\
\hline A_51_P455866 & 1419555_at \\
\hline A_55_P2117119 & 1448507_at \\
\hline A_51_P437135 & 1421100_a_at \\
\hline A_52_P376169 & 1457843_at \\
\hline A_55_P2091858 & 1425983_x_at \\
\hline A_52_P598634 & 1429260_at \\
\hline A_55_P1960108 & 1437418_at \\
\hline A_52_P250400 & 1424701_at \\
\hline A_51_P355427 & 1450974_at \\
\hline A_66_P139785 & 1448908_at \\
\hline A_55_P2027900 & 1421738_at \\
\hline A_51_P352452 & 1443525_at \\
\hline A_51_P170463 & 1456833_at \\
\hline A_55_P2100884 & 1422733_at \\
\hline A_55_P2030373 & 1452308_a_at \\
\hline A_51_P436727 & 1460444_at \\
\hline A_51_P481159 & 1427912_at \\
\hline A_55_P1982454 & 1422824_s_at \\
\hline A_55_P2094831 & 1423343_at \\
\hline A_52_P288251 & 1436698_x_at \\
\hline A_52_P12877 & 1431182_at \\
\hline A_55_P1965931 & 1418013_at \\
\hline A_51_P185247 & 1424007_at \\
\hline A_55_P1991164 & 1448139_at \\
\hline A_51_P355427 & 1435833_at \\
\hline A_55_P1974645 & 1418259_a_at \\
\hline A_55_P2003483 & 1416049_at \\
\hline A_52_P249424 & 1420909_at \\
\hline A_30_P01021563 & 1457257_x_at \\
\hline A_51_P224042 & 1453310_at \\
\hline A_55_P2010871 & 1455717_s_at \\
\hline A_51_P171288 & 1455154_at \\
\hline A_52_P85174 & 1422781_at \\
\hline A_55_P1963154 & 1450154_at \\
\hline A_55_P2027083 & 1419601_at \\
\hline A_55_P2012241 & 1433501_at \\
\hline A_55_P1965616 & 1436670_x_at \\
\hline A_51_P255682 & 1420911_a_at \\
\hline A_52_P195881 & 1428536_at \\
\hline A_52_P512575 & 1451776_s_at \\
\hline A_51_P434670 & 1425597_a_at \\
\hline
\end{tabular}




\section{) acutely isolated astrocytes \\ AGILENT Gene Symbol}

Gjb6

Dio2

Ppp1r3g

Grin2c

Etnppl

Al464131

S100b

Gpc5

Adora2b

2900052N01Rik

Timp4

Cyp4f15

Cyp4f14

$\mathrm{Ntsr} 2$

Fam20a

Etnppl

Slc7a10

Slc1a2

Unc13c

Hsd11b1

Bcan

Ptger4

Ttpa

S100b

Chrdl1

Fam107a

Prss56

2610034M16Rik

Chrdl1

Pygm

Gpld1

Sult1a1

Tph2

Tril

Hhatl

Cdh19

Lgi4

Tril

Aifm3

Prex2

Nrarp

Gm11627

Abhd3

Eva1a
AFFY Gene Symbol

Gjb6

Dio2

Ppp1r3g

Grin2c

Al464131

S100b

Gpc5

Adora2b

2900052N01Rik

Timp4

Cyp4f15

Cyp4f14

Ntsr2

Fam20a

Etnppl

Slc7a10

Slc1a2

Unc13c

Hsd11b1

Bcan

Ptger4

Ttpa

S100b

Chrdl1

Fam107a

Prss56

2610034M16Rik

Chrdl1

Pygm

Gpld1

Sult1a1

Tph2

Tril

Hhatl

Cdh19

Lgi4

Tril

Aifm3

Prex2

Nrarp

Gm11627

Abhd3

Eva1a q-value

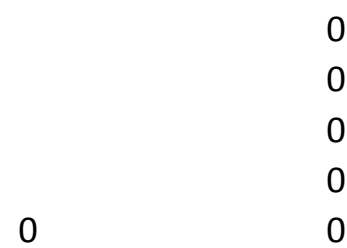

0
0
0
0

0

0

0

0

0

0

0

0

0

0

0

0

0

0

0

0

0

0

0

0

0

0

0

0

0

0

0

0

0

0

5.85E-05

5.85E-05

5.85E-05

5.85E-05

5.85E-05

5.85E-05

5.85E-05

5.85E-05 


\begin{tabular}{|c|c|c|}
\hline Fam107a & Fam107a & $5.85 \mathrm{E}-05$ \\
\hline S1pr1 & S1pr1 & $5.85 \mathrm{E}-05$ \\
\hline Phkg1 & Phkg1 & $5.85 \mathrm{E}-05$ \\
\hline Hes5 & Hes5 & $5.85 \mathrm{E}-05$ \\
\hline Kcnj16 & Kcnj16 & $5.85 \mathrm{E}-05$ \\
\hline Cep128 & Cep128 & $5.85 \mathrm{E}-05$ \\
\hline Slc39a12 & Slc39a12 & $5.85 \mathrm{E}-05$ \\
\hline Cyp2d22 & Cyp2d22 & $5.85 \mathrm{E}-05$ \\
\hline Myoc & Myoc & $5.85 \mathrm{E}-05$ \\
\hline Phkg1 & Phkg1 & $5.85 \mathrm{E}-05$ \\
\hline Gabrg1 & Gabrg1 & $5.85 \mathrm{E}-05$ \\
\hline Eogt & Eogt & $5.85 \mathrm{E}-05$ \\
\hline Smim3 & Smim3 & 1.67E-04 \\
\hline Cmtm5 & Cmtm5 & $1.67 \mathrm{E}-04$ \\
\hline Tlcd1 & Tlcd1 & $1.67 \mathrm{E}-04$ \\
\hline Aldoc & Aldoc & $1.99 \mathrm{E}-04$ \\
\hline \multirow[t]{2}{*}{ Cep128 } & Cep128 & $1.99 \mathrm{E}-04$ \\
\hline & o 4933416E14Rik & $1.99 \mathrm{E}-04$ \\
\hline Fam107a & Fam107a & $1.99 \mathrm{E}-04$ \\
\hline Tlcd1 & Tlcd1 & $1.99 \mathrm{E}-04$ \\
\hline Tlr3 & Tlr3 & 1.99E-04 \\
\hline Tmem229a & Tmem229a & $1.99 \mathrm{E}-04$ \\
\hline Pla2g7 & Pla2g7 & $2.94 \mathrm{E}-04$ \\
\hline Slc13a5 & Slc13a5 & $3.38 \mathrm{E}-04$ \\
\hline Slc39a12 & Slc39a12 & $3.70 \mathrm{E}-04$ \\
\hline Ppp1r3d & Ppp1r3d & $3.70 \mathrm{E}-04$ \\
\hline Slc30a10 & Slc30a10 & $3.70 \mathrm{E}-04$ \\
\hline Chrdl1 & Chrdl1 & $3.85 \mathrm{E}-04$ \\
\hline ॥18 & $\| 18$ & $3.85 \mathrm{E}-04$ \\
\hline Omg & Omg & $3.85 \mathrm{E}-04$ \\
\hline Hepacam & Hepacam & $3.85 \mathrm{E}-04$ \\
\hline Gabra2 & Gabra2 & $3.85 \mathrm{E}-04$ \\
\hline Gabrg1 & Gabrg1 & $3.85 \mathrm{E}-04$ \\
\hline Slc25a18 & Slc25a18 & 4.17E-04 \\
\hline Gpr37|1 & Gpr37l1 & 4.17E-04 \\
\hline Slc1a3 & Slc1a3 & 4.37E-04 \\
\hline Ptger4 & Ptger4 & 4.37E-04 \\
\hline Maob & Maob & 4.37E-04 \\
\hline Acsbg1 & Acsbg1 & $4.37 \mathrm{E}-04$ \\
\hline Fzd2 & Fzd2 & 4.71E-04 \\
\hline Aldh1a1 & Aldh1a1 & $6.13 \mathrm{E}-04$ \\
\hline Car8 & Car8 & 6.13E-04 \\
\hline Myom3 & Myom3 & $6.44 \mathrm{E}-04$ \\
\hline Slc1a2 & Slc1a2 & $6.74 \mathrm{E}-04$ \\
\hline Tmem229a & Tmem229a & $7.41 \mathrm{E}-04$ \\
\hline Slc14a1 & Slc14a1 & 7.69E-04 \\
\hline Aifm3 & Aifm3 & 8.16E-04 \\
\hline
\end{tabular}




Lypd6
Tmem100
Ltbp1
Cyp2j9
G0s2
Eogt
Fzd2
Thbs4
A730056I06Rik
Akt2
Gm5089
Slc1a3
Unc13c
Selenbp1
Pnpla7
Ephx2
Kctd14
Itih3
Phka1
Fgfr3
Phka1
Papss2

Lypd6

Tmem100

8.16E-04

Ltbp1

Cyp2j9

8.16E-04

9.03E-04

G0s2

9.03E-04

Eogt

9.15E-04

Fzd2

9.15E-04

Thbs4

9.15E-04

A730056I06Rik

9.15E-04

Akt2

9.15E-04

Gm5089

9.15E-04

Slc1a3

Unc13c

Selenbp1

Pnpla7

Ephx2

9.94E-04

9.94E-04

9.97E-04

9.97E-04

9.97E-04

Kctd14

0.00100295

Itih3

0.00100295

Phka1

0.00100295

Fgfr3

0.00100295

Phka1

0.00100295

0.00100295

Papss2

0.001014493

II33

Agmo

II33

0.001014493

Agmo

0.001016949

Egfl6

Egfl6

0.001016949

Apln

Apln

0.001016949

F3

Phactr3

F3

0.001288515

Phactr3

0.001305556

Atp1a2

Atp1a2

0.001338798

Nat8

Slc27a1

Nat8

0.001338798

Slc27a1

0.001490515

Acot11

0.001435897

Aqp4

0.001435897

Aqp4

Thrsp

0.001435897

Kcnj16

0.001435897

Kcnj16

Cntfr

0.001435897

Nrros

Nrros

0.001435897

0 Rorb

0.001435897

HIf

HIf

Cd38

0.001450382

Cd38

Fgd6

0.001464646

Fgd6

SIc4a4

Slc4a4

0.001519608

Rfx4

0.001519608

Rfx4

Lypd6

Lypd6

0.001519608

Gja1

0.001519608

Gja1

Bco2

Bco2

0.001545894

Gjb2

0.001545894

0.001558753 


\begin{tabular}{|c|c|c|}
\hline \multirow[t]{2}{*}{ St6galnac5 } & St6galnac5 & 0.001571429 \\
\hline & $0 \mathrm{Gm} 2115$ & 0.001619718 \\
\hline \multirow[t]{2}{*}{ Chst1 } & Chst1 & 0.001619718 \\
\hline & 0 Chpt1 & 0.001757991 \\
\hline Cth & Cth & 0.001757991 \\
\hline Phka1 & Phka1 & 0.001757991 \\
\hline Eps8 & Eps8 & 0.001757991 \\
\hline Adhfe1 & Adhfe1 & 0.001756757 \\
\hline Selenbp1 & Selenbp1 & 0.001756757 \\
\hline Aqp4 & Aqp4 & 0.001834452 \\
\hline Myo6 & Myo6 & 0.00187638 \\
\hline St6galnac5 & LOC552874 | St6galnac5 & 0.00187638 \\
\hline Hes5 & Hes5 & 0.001873638 \\
\hline Gm6556 & Gm6556 & 0.001873638 \\
\hline Elovl2 & Elovl2 & 0.001991342 \\
\hline Kctd14 & Kctd14 & 0.002086022 \\
\hline Slc9a3r1 & Slc9a3r1 & 0.002067511 \\
\hline Ppara & Ppara & 0.002067511 \\
\hline Lsamp & Lsamp & 0.002067511 \\
\hline Tmem51 & Tmem51 & 0.002180293 \\
\hline Bmpr1b & Bmpr1b & 0.0021875 \\
\hline Gpt & Gpt & 0.002283951 \\
\hline Scrg1 & Scrg1 & 0.002283951 \\
\hline Tmem144 & Tmem144 & 0.002357724 \\
\hline Usp54 & Usp54 & 0.002357724 \\
\hline Pamr1 & Pamr1 & 0.002383838 \\
\hline Hepacam & Hepacam & 0.002409639 \\
\hline Fgfr3 & Fgfr3 & 0.00247505 \\
\hline 1700019G17Rik & 1700019G17Rik & 0.002480159 \\
\hline Tfcp2/1 & Tfcp2I1 & 0.002509804 \\
\hline Naaa & Naaa & 0.002509804 \\
\hline Cbs & Cbs & 0.002534113 \\
\hline Eogt & Eogt & 0.00253876 \\
\hline Nkain4 & Nkain4 & 0.002681992 \\
\hline \multirow[t]{2}{*}{ Vcam1 } & Vcam1 & 0.002681992 \\
\hline & 0 Phgdh & 0.002685714 \\
\hline Gpr37 & Gpr37 & 0.002689394 \\
\hline Hipk2 & Hipk2 & 0.002749529 \\
\hline Oaf & Oaf & 0.002752809 \\
\hline Adcy2 & Adcy2 & 0.002811918 \\
\hline Car2 & Car2 & 0.002799264 \\
\hline Gprc5b & Gprc5b & 0.002799264 \\
\hline Gramd3 & Gramd3 & 0.002893773 \\
\hline HIf & HIf & 0.003005465 \\
\hline Dnaic1 & Dnaic1 & 0.003007246 \\
\hline Kcnn2 & Kcnn2 & 0.003028674 \\
\hline Sec14l2 & Sec14I2 & 0.003028674 \\
\hline
\end{tabular}




Rlbp1
Kctd14
Eya1
Naaa
Elf5
Efhd1
Dab1
Lypd6
Hipk2
1190007l07Rik
Pcdh20
Timp4
Ppap2b
Gabra2
Dbx2
Gpr17
Fix1

Rlbp1
Kctd14
Eya1
Naaa
Elf5

Efhd1

Dab1

Lypd6

Hipk2

1810014B01Rik

0 Gm3515 | Rmst | Rmst

Pcdh20

Timp4

Ppap2b

Gabra2

Dbx2

Gpr17

Fjx1

Atp1a2

Arrb1

Cbr3

Eps8

Slco1c1

Tmem204

Hspa8

Cml1

Gdf10

Mlc1

Mlc1

Timp4

Entpd2

Gldc

Vegfa

Ppil6

Daam2

Gli3

TIr3

Folh1

Kcnj10

Ctso

1700019G17Rik

Mfge8

Kcng4

Hopx

Qk
0.003065954

0.003191489

0.003227513

0.003245614

0.003246073

0.00328125

0.00338488

0.00338488

0.003434856

0.003434856

0.003434856

0.003451179

0.003500838

0.003582089

0.003582089

0.003596059

0.003596059

0.003788618

0.003788618

0.00381877

0.003864734

0.003974359

0.004051037

0.004063492

0.004060031

0.004166667

0.004178404

0.004205607

$0 \quad 0.004178187$

0.004178187

0.004178187

0.004272727

0.004272727

0.004272727

0.004519519

0.004519519

0.004538691

0.004538691

0.004592592

0.004631268

0.004625551

0.004649123

0.004905386

0.004942029

0.004978355 


\section{RP-value}

24.644915

26.695889

27.522926

33.58823

43.206196

61.288677

62.309135

62.836388

64.41103

69.695885

69.91839

71.759315

72.41244

73.50246

75.417435

83.77455

94.02736

94.02997

103.75071

106.39344

108.31859

112.75734

113.002426

116.54206

124.52192

125.80281

127.0251

136.35811

139.29944

141.49802

142.41406

142.93747

143.23853

147.61919

149.85312

152.98512

158.6776

166.2588

167.47302

168.47269

169.36873

171.65129

172.2331

175.3014 


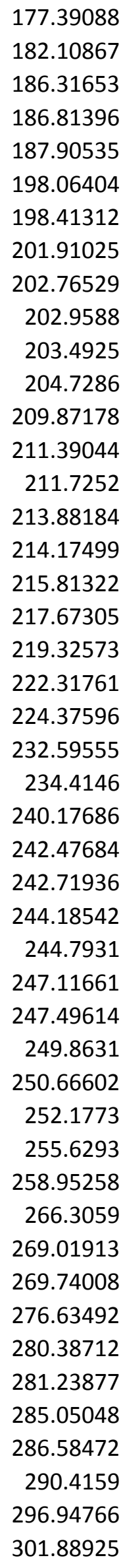


302.3299

303.2233

307.74292

308.7409

310.9743

313.14096

313.46243

313.74786

317.3688

317.8436

319.70502

321.36166

323.77533

324.88193

325.97708

330.44644

332.7287

333.43552

335.09216

335.79083

336.33533

338.11765

338.93024

342.0699

342.99194

343.24338

350.84372

353.40848

357.3133

357.7657

362.4375

362.84885

365.73975

368.32013

368.90146

369.5671

370.3712

370.90448

373.62262

376.78854

379.4678

379.59576

379.71426

380.60217

381.85162

382.44742

384.4294 
384.6726

386.04007

387.0892

396.22092

397.13812

397.1438

397.16418

400.7253

400.85852

404.258

407.03204

407.91922

408.72513

409.5564

414.5429

418.4479

419.91495

419.9687

421.51254

425.69516

428.70026

431.65393

431.71558

433.46893

434.47955

435.32947

438.09555

439.92416

441.28415

443.32953

444.2281

444.37793

446.0623

451.50034

451.54767

453.3818

454.6325

457.33798

458.19244

461.158

461.62555

462.0157

463.79324

466.17126

467.1176

468.19165

468.2247

https://mc04.manuscriptcentral.com/cares 


$$
\begin{array}{r}
468.82596 \\
474.52576 \\
475.7964 \\
476.19968 \\
479.54742 \\
480.16354 \\
485.2855 \\
485.30936 \\
488.38437 \\
488.5409 \\
488.7794 \\
489.06485 \\
491.48804 \\
494.9017 \\
495.88098 \\
496.7033 \\
497.11383 \\
501.4433 \\
501.52478 \\
503.03995 \\
505.13177 \\
509.17627 \\
512.2961 \\
512.92303 \\
514.40344 \\
516.1878 \\
517.0673 \\
518.43933 \\
519.4627 \\
519.7193 \\
519.98596 \\
523.78125 \\
523.8691 \\
523.92285 \\
531.1601 \\
531.2857 \\
533.3345 \\
533.9539 \\
536.3649 \\
537.08685 \\
537.97784 \\
538.7643 \\
543.3847 \\
548.8445
\end{array}
$$




\section{Ranked list of genes up-regulated in non-Tg astrocytes com|} AGILENT SurePrint G3 GE 8x60k probc AFFY Mouse430 2 probe

\begin{tabular}{|c|c|}
\hline A_52_P622850 & 1456010_x_at \\
\hline A_52_P150950 & 1452090_a_at \\
\hline A_55_P2045299 & 1457536_at \\
\hline A_51_P274488 & 1417051_at \\
\hline A_51_P396883 & 1460408_at \\
\hline A_55_P2113981 & 1434819_at \\
\hline A_55_P2130249 & 1418791_at \\
\hline A_51_P274488 & 1447825_x_at \\
\hline A_55_P2172470 & 1423231_at \\
\hline A_52_P883557 & 1438751_at \\
\hline A_55_P1990309 & 1452894_at \\
\hline A_52_P150950 & 1425898_x_at \\
\hline A_55_P2051849 & 1416828_at \\
\hline A_52_P309166 & 1419420_at \\
\hline A_55_P1992049 & 1434141_at \\
\hline A_55_P2061620 & 1449147_at \\
\hline A_51_P127297 & 1449038_at \\
\hline A_52_P515880 & 1420955_at \\
\hline A_66_P108810 & 1449465_at \\
\hline A_55_P1972981 & 1429651_at \\
\hline A_55_P1997525 & 1419028_at \\
\hline A_52_P568235 & 1439904_at \\
\hline A_55_P2057132 & 1423515_at \\
\hline A_52_P229959 & 1420877_at \\
\hline A_51_P394997 & 1418047_at \\
\hline A_55_P2027900 & 1455444_at \\
\hline A_52_P298394 & 1423183_at \\
\hline A_55_P1954486 & 1424719_a_at \\
\hline A_55_P1990309 & 1428741_at \\
\hline A_55_P2164629 & 1416211_a_at \\
\hline A_51_P509643 & 1436853_a_at \\
\hline A_52_P38208 & 1424248_at \\
\hline A_55_P1977431 & 1419473_a_at \\
\hline A_55_P2062737 & 1416444_at \\
\hline A_55_P2075298 & 1433965_at \\
\hline A_55_P1962303 & 1428361_x_at \\
\hline A_51_P396883 & 1427227_at \\
\hline A_55_P1984307 & 1440225_at \\
\hline A_52_P560006 & 1455374_at \\
\hline A_52_P179068 & 1420872_at \\
\hline A_55_P1954034 & 1441317_x_at \\
\hline A_51_P187352 & 1455554_at \\
\hline A_55_P2143688 & 1458492_x_at \\
\hline A_52_P124472 & 1422835_at \\
\hline
\end{tabular}


A_55_P1954486

A_51_P154379

A_51_P256384

A_51_P359173

A_51_P259603

A_51_P249193

A_55_P1976278

A_55_P1993777

A_55_P2011290

A_55_P2143688

A_52_P356093

A_52_P250400

A_55_P2132512

A_55_P2037662

A_55_P1953753

A_55_P1969650

A_51_P463401

A_52_P309166

A_51_P270355

A_55_P2013601

A_52_P655687

A_52_P377537

A_52_P26357

A_51_P268193

A_66_P112684

A_52_P531731

A_66_P112684

A_55_P2001793

A_55_P2020602

A_51_P187352

A_52_P883557

A_55_P2105472

A_55_P1962303

A_51_P222993

A_55_P2027900

A_55_P2094831

A_52_P282500

A_55_P2009752

A_51_P189814

A_55_P2143688

A_52_P72965

A_51_P477458

A_52_P201558

A_55_P1965694

A_55_P1956567

A_52_P309166

A_55_P2236268

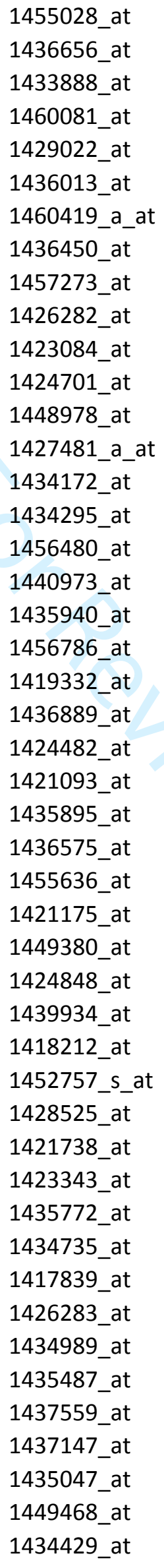




\begin{tabular}{|c|c|}
\hline A_55_P1982454 & 1422823_at \\
\hline A_51_P237255 & 1436275_at \\
\hline A_52_P40695 & 1435308_at \\
\hline A_55_P2046744 & 1450713_at \\
\hline A_66_P118059 & 1450466_at \\
\hline A_52_P136782 & 1417466_at \\
\hline A_52_P221756 & 1435026_at \\
\hline A_51_P233153 & 1451499_at \\
\hline A_55_P1953391 & 1418314_a_at \\
\hline A_55_P2143499 & 1460570_at \\
\hline A_55_P2013888 & 1438782_at \\
\hline A_55_P2001793 & 1436483_at \\
\hline A_52_P66226 & 1417529_at \\
\hline A_55_P2427900 & 1455304_at \\
\hline A_52_P8324 & 1429175_at \\
\hline A_52_P622850 & 1423146_at \\
\hline A_55_P1982454 & 1422824_s_at \\
\hline A_52_P628915 & 1415844_at \\
\hline A_55_P2014229 & 1448110_at \\
\hline A_55_P2108151 & 1417184_s_at \\
\hline A_51_P509643 & 1418493_a_at \\
\hline A_55_P2164629 & 1448254_at \\
\hline A_51_P438657 & 1439808_at \\
\hline A_51_P337523 & 1455462_at \\
\hline A_55_P2075298 & 1454728_s_at \\
\hline A_51_P488180 & 1434759_at \\
\hline A_55_P2057132 & 1457412_at \\
\hline A_55_P1954693 & 1416783_at \\
\hline A_55_P2075298 & 1423597_at \\
\hline A_52_P494622 & 1455034_at \\
\hline A_66_P123643 & 1423471_at \\
\hline A_55_P2086213 & 1435957_at \\
\hline A_52_P265937 & 1450683_at \\
\hline A_55_P2332926 & 1428866_at \\
\hline A_51_P359173 & 1441927_at \\
\hline A_55_P2148748 & 1446190_at \\
\hline A_55_P2012572 & 1456392_at \\
\hline A_52_P201558 & 1457743_at \\
\hline A_55_P2078040 & 1460608_at \\
\hline A_52_P298394 & 1435851_at \\
\hline A_55_P2018176 & 1455161_at \\
\hline A_55_P2013601 & 1439557_s_at \\
\hline A_51_P109840 & 1420484_a_at \\
\hline A_51_P477458 & 1421436_at \\
\hline
\end{tabular}




\begin{tabular}{|c|c|c|}
\hline AGILENT Gene Symbol & AFFY Gene Symbol & q-value \\
\hline Hes5 & Hes5 & 0 \\
\hline Olfm3 & Olfm3 & 0 \\
\hline Gpc5 & Gpc5 & 0 \\
\hline Pcdh8 & Pcdh8 & 0 \\
\hline Gabrg1 & Gabrg1 & 0 \\
\hline St6gal2 & St6gal2 & 0 \\
\hline Sh3gl2 & Sh3gl2 & 0 \\
\hline Pcdh8 & Pcdh8 & 0 \\
\hline Nrgn & Nrgn & 0 \\
\hline Slc30a10 & Slc30a10 & 0 \\
\hline Elav14 & Elavl4 & 0 \\
\hline Olfm3 & Olfm3 & 0 \\
\hline Snap25 & Snap25 & 0 \\
\hline St6galnac5 & St6galnac5 & 0 \\
\hline Gucy1a3 & Gucy1a3 & 0 \\
\hline Chst1 & Chst1 & 0 \\
\hline Hsd11b1 & Hsd11b1 & 0 \\
\hline Vsnl1 & Vsnl1 & $1.85 \mathrm{E}-04$ \\
\hline Reln & Reln & $1.85 \mathrm{E}-04$ \\
\hline Phactr3 & Phactr3 & $1.85 \mathrm{E}-04$ \\
\hline Arpp21 & Arpp21 & $1.85 \mathrm{E}-04$ \\
\hline Fstl5 & Fstl5 & $1.85 \mathrm{E}-04$ \\
\hline Scn8a & Scn8a & $1.85 \mathrm{E}-04$ \\
\hline Sept6 & Sept6 & $1.85 \mathrm{E}-04$ \\
\hline Neurod6 & Neurod6 & $1.85 \mathrm{E}-04$ \\
\hline Gabra2 & Gabra2 & $1.85 \mathrm{E}-04$ \\
\hline Lgi1 & Lgi1 & $1.85 \mathrm{E}-04$ \\
\hline Mapt & Mapt & $1.85 \mathrm{E}-04$ \\
\hline Elavl4 & Elavl4 & $1.85 \mathrm{E}-04$ \\
\hline Ptn & Ptn & $2.22 \mathrm{E}-04$ \\
\hline Snca & Snca & $2.22 \mathrm{E}-04$ \\
\hline Arpp21 & Arpp21 & $2.22 \mathrm{E}-04$ \\
\hline Cck & Cck & $2.22 \mathrm{E}-04$ \\
\hline Elovl2 & Elovl2 & $2.22 \mathrm{E}-04$ \\
\hline Atp8a1 & Atp8a1 & $2.22 \mathrm{E}-04$ \\
\hline Hba-a1 & Hba-a1 | Hba-a2 & 3.70E-04 \\
\hline Gabrg1 & Gabrg1 & $3.70 \mathrm{E}-04$ \\
\hline Gpr116 & Gpr116 & 4.39E-04 \\
\hline Kcnj3 & Kcnj3 & $5.13 \mathrm{E}-04$ \\
\hline Gucy1b3 & Gucy1b3 & $5.13 \mathrm{E}-04$ \\
\hline Jakmip1 & Jakmip1 & 5.13E-04 \\
\hline Kcnma1 & A830039N20Rik & 5.56E-04 \\
\hline $\mathrm{Ntm}$ & $\mathrm{Ntm}$ & $6.20 \mathrm{E}-04$ \\
\hline Kcnd2 & Kcnd2 & $6.82 \mathrm{E}-04$ \\
\hline
\end{tabular}


Mapt

Tmem150c

Atp2b2

Syt7

Adcyap1r1

Gsg1l

Prkcb

Rbfox3

Tenm2

$\mathrm{Ntm}$

B3galt2

Pcdh20

Ngef

Atp1a3

Cnr1

Rasgrp1

Fry

St6galnac5

Dclk1

Ldb2

Egfl6

Gabra1

Arhgef7

Slc7a10

Lsamp

Grin3a

Lsamp

Myt1l

Pacsin1

Kcnma1

Slc30a10

Omg

Hba-a1

Pnmal1

Gabra2

Slco1c1

Kif21b

HIf

Cldn5

Ntm

Unc80

Grid2

Rgs7bp

Gabrg2

Rab3c

St6galnac5

Syt16
Mapt

6.82E-04

Tmem150c

8.70E-04

Atp2b2

0.00106383

Syt7

0.00106383

Adcyap1r1

0.00106383

Gsg1l

Prkcb

0.001133333

Rbfox3

Tenm2

Ntm

B3galt2

Pcdh20

Ngef

Atp1a3

Cnr1

Rasgrp1

Fry

LOC552874 | St6galnac5

Dclk1

Ldb2

Egfl6

Gabra1

Arhgef7

Slc7a10

Lsamp

Grin3a

Lsamp

Myt1I

Pacsin1

Kcnma1

Slc30a10

Omg

Hba-a1 | Hba-a2

4930488B01Rik | Pnmal1

Gabra2

Slco1c1

Kif21b

HIf

Cldn5

Ntm

Unc80

Grid2

Rgs7bp

Gabrg2

Rab3c

St6galnac5

Syt16
0.001133333

0.001133333

0.001133333

0.001133333

0.001133333

0.001133333

0.001133333

0.001133333

0.001073446

0.001222222

0.001256831

0.001290323

0.001290323

0.001302083

0.001302083

0.001302083

0.001302083

0.00127451

0.00127451

0.001476191

0.001690141

0.001851852

0.001851852

0.001936937

0.001955556

0.002017544

0.002350427

0.002489452

0.002541667

0.00255144

0.00255144

0.002570281

0.002570281

0.002570281

0.00251938

0.002528736

0.002528736

0.00258427

0.00258427

0.002564103
0.002207792 


\begin{tabular}{|c|c|c|}
\hline Eps8 & Eps8 & 0.002572464 \\
\hline Kcnip2 & Kcnip2 & 0.002724014 \\
\hline Fut9 & Fut9 & 0.003049645 \\
\hline Cspg5 & Cspg5 & 0.003087719 \\
\hline Cdk5r2 & Cdk5r2 & 0.003090278 \\
\hline Rgs5 & Rgs5 & 0.003090278 \\
\hline Spock2 & Spock2 & 0.003061225 \\
\hline Cadps2 & Cadps2 & 0.003131313 \\
\hline Rbfox1 & Rbfox1 & 0.003166667 \\
\hline Pgbd5 & Pgbd5 & 0.003168317 \\
\hline Cntn4 & Cntn4 & 0.003168317 \\
\hline Myt1l & Myt1l & 0.003139159 \\
\hline Rab33a & Rab33a & 0.003141026 \\
\hline Unc13c & Unc13c & 0.003141026 \\
\hline Tmem178 & Tmem178 & 0.003144654 \\
\hline Hes5 & Hes5 & 0.003302181 \\
\hline Eps8 & Eps8 & 0.003487654 \\
\hline Syt4 & Syt4 & 0.00351682 \\
\hline Sema4a & Sema4a & 0.00351682 \\
\hline Hbb-b1 & Hbb-b1 | Hbb-b2 | Hbb-bs | Hbb-bt & 0.00351682 \\
\hline Snca & Snca & 0.003452381 \\
\hline Ptn & Ptn & 0.00359882 \\
\hline Ipcef1 & Ipcef1 & 0.00371345 \\
\hline Adcy2 & Adcy2 & 0.00371345 \\
\hline Atp8a1 & Atp8a1 & 0.003706896 \\
\hline Lrrtm3 & Lrrtm3 & 0.003732194 \\
\hline Scn8a & Scn8a & 0.0040113 \\
\hline Tac1 & Tac1 & 0.0040113 \\
\hline Atp8a1 & Atp8a1 & 0.004194445 \\
\hline $\mathrm{Nr} 4 \mathrm{a} 2$ & $\mathrm{Nr} 4 \mathrm{a} 2$ & 0.004269972 \\
\hline Ptbp2 & Ptbp2 & 0.004371585 \\
\hline Kcnq3 & B830032F12 | Kcnq3 & 0.004371585 \\
\hline Tagln3 & Tagln3 & 0.004354839 \\
\hline 2810037022Rik & 2810037022Rik & 0.0044 \\
\hline Syt7 & Syt7 & 0.004417989 \\
\hline Dclk1 & Dclk1 & 0.004435696 \\
\hline Negr1 & Negr1 & 0.004427083 \\
\hline Rgs7bp & 0 & 0.004418605 \\
\hline Cacna1b & Cacna1b & 0.004435897 \\
\hline Lgi1 & Lgi1 & 0.004435897 \\
\hline Al504432 & Al504432 & 0.004671717 \\
\hline Ldb2 & Ldb2 & 0.004786967 \\
\hline Vtn & Vtn & 0.004900497 \\
\hline Grid2 & Grid2 & 0.005 \\
\hline
\end{tabular}




\section{RP-value}

38.228867

40.14826

45.530594

62.825222

63.489967

70.426056

72.795235

75.77436

77.42768

77.601295

78.4775

79.4883

85.087296

85.21892

86.613976

86.902824

94.64061

107.83927

108.764145

110.133865

110.722885

113.11523

113.57372

120.68429

124.78282

124.88154

128.00078

129.13066

129.43092

131.86943

132.60532

135.56566

138.43987

138.47098

139.53728

143.77821

153.75385

156.69489

157.85565

162.09242

163.97165

167.04994

169.75223

176.13744 


$$
\begin{aligned}
& 179.00458 \\
& 186.50255 \\
& 189.62704 \\
& 190.53268 \\
& 190.71278 \\
& 192.45544 \\
& 195.93103 \\
& 197.03783 \\
& 197.31279 \\
& 197.84146 \\
& 198.58455 \\
& 201.48653 \\
& 202.16022 \\
& 202.53145 \\
& 203.75371 \\
& 206.5847 \\
& 209.93912 \\
& 211.64128 \\
& 212.32985 \\
& 213.5691 \\
& 213.70602 \\
& 215.07327 \\
& 216.10878 \\
& 217.23347 \\
& 217.45518 \\
& 233.61598 \\
& 243.20815 \\
& 247.11243 \\
& 247.14339 \\
& 249.70981 \\
& 251.43748 \\
& 253.3812 \\
& 257.16528 \\
& 268.65164 \\
& 275.85614 \\
& 277.00516 \\
& 277.91016 \\
& 278.34482 \\
& 281.2168 \\
& 281.3428 \\
& 282.6356 \\
& 284.27158 \\
& 287.12534 \\
& 287.5331 \\
& 289.21994 \\
& 291.4899 \\
& 293.7496
\end{aligned}
$$




$$
\begin{array}{r}
295.9357 \\
299.10336 \\
307.84622 \\
310.2925 \\
311.68964 \\
311.8501 \\
311.99994 \\
312.87704 \\
314.05276 \\
315.99564 \\
316.35825 \\
317.2488 \\
319.63144 \\
319.81186 \\
321.23337 \\
324.1635 \\
329.6535 \\
330.8359 \\
330.9058 \\
332.35577 \\
333.18903 \\
336.3066 \\
339.2922 \\
340.9835 \\
341.86188 \\
343.79172 \\
349.89343 \\
349.94983 \\
353.92282 \\
355.45477 \\
356.17947 \\
356.44672 \\
358.22116 \\
358.854 \\
360.29373 \\
360.94888 \\
361.6654 \\
363.14523 \\
364.91345 \\
364.97015 \\
370.66614 \\
374.21698 \\
375.81137 \\
382.1414 \\
\hline
\end{array}
$$




\section{Ranked list of genes down-regulated in non-Tg astrocytes $\mathrm{Cl}$} AGILENT SurePrint G3 GE 8x60k probı AFFY Mouse430 2 probe

A_55_P2007155

A_55_P2092826

A_51_P295085

A_52_P278336

A_55_P2085142

A_55_P2056325

A_51_P207622

A_55_P2017418

A_51_P404463

A_55_P2032678

A_55_P1990111

A_52_P679105

A_55_P2039284

A_55_P2087607

A_51_P405606

A_52_P292792

A_52_P175376

A_51_P450527

A_55_P2017636

A_51_P405606

A_51_P207622

A_55_P2111302

A_55_P2017418

A_51_P207622

A_51_P465281

A_55_P2146254

A_55_P2039284

A_66_P111562

A_55_P2051681

A_55_P2087607

A_55_P2115798

A_52_P220879

A_51_P427663

A_51_P405606

A_51_P341736

A_30_P01023502

A_52_P185907

A_52_P220879

A_55_P2055487

A_55_P1963154

A_51_P472726

A_52_P220879

A_66_P114451

A_52_P93837 1418979_at

1448213_at

1419663_at

1416164_at

1449254_at

1460330_at

1456084_x_at

1450876_at

1460049_s_at

1416121_at

1422629_s_at

1431057_a_at

1425964_x_at

1417494_a_at

1456174_x_at

1455627_at

1418091_at

1423505_at

1421811_at

1423413_at

1437718_x_at

1448735_at

1423153_x_at

1437685_x_at

1419573_a_at

1424254_at

1422943_a_at

1417419_at

1424885_at

1417495_x_at

1426260_a_at

1455900_x_at

1450981_at

1420760_s_at

1439364_a_at

1421375_a_at

1448326_a_at

1433428_x_at

1426210_x_at

1450154_at

1423946_at

1437277_x_at

1437409_s_at

1455961_at 


\begin{tabular}{|c|c|}
\hline A_51_P404463 & 1423261_at \\
\hline A_55_P2087607 & 1448734_at \\
\hline A_55_P2110713 & 1419091_a_at \\
\hline A_51_P131408 & 1418572_x_at \\
\hline A_51_P465281 & 1455439_a_at \\
\hline A_51_P515605 & 1427883_a_at \\
\hline A_66_P111562 & 1448698_at \\
\hline A_55_P2055487 & 1451969_s_at \\
\hline A_51_P342567 & 1419706_a_at \\
\hline A_51_P222657 & 1438948_x_at \\
\hline A_55_P2064043 & 1423760_at \\
\hline A_66_P104815 & 1448613_at \\
\hline A_55_P2016647 & 1448314_at \\
\hline A_55_P2459897 & 1434719_at \\
\hline A_51_P414889 & 1445897_s_at \\
\hline A_55_P1952379 & 1416125_at \\
\hline A_52_P263095 & 1452217_at \\
\hline A_51_P315682 & 1439764_s_at \\
\hline A_55_P1980631 & 1451246_s_at \\
\hline A_52_P304720 & 1418476_at \\
\hline A_51_P376238 & 1416625_at \\
\hline A_55_P2033997 & 1439096_at \\
\hline A_51_P222657 & 1456251_x_at \\
\hline A_55_P2003033 & 1446951_at \\
\hline A_51_P405606 & 1450976_at \\
\hline A_55_P2025033 & 1452242_at \\
\hline A_55_P1997604 & 1448558_a_at \\
\hline A_30_P01030135 & 1424768_at \\
\hline A_51_P131408 & 1418571_at \\
\hline A_55_P2154709 & 1417667_a_at \\
\hline A_55_P1995205 & 1454694_a_at \\
\hline A_55_P2104894 & 1422437_at \\
\hline A_52_P413646 & 1450759_at \\
\hline A_52_P602847 & 1424825_a_at \\
\hline A_52_P372151 & 1420930_s_at \\
\hline A_51_P157042 & 1416953_at \\
\hline A_52_P223127 & 1435399_at \\
\hline A_55_P2086949 & 1422033_a_at \\
\hline A_51_P222657 & 1416695_at \\
\hline A_52_P52303 & 1440142_s_at \\
\hline A_51_P484111 & 1455978_a_at \\
\hline A_55_P2002577 & 1422438_at \\
\hline A_51_P464387 & 1417013_at \\
\hline A_30_P01029470 & 1460603_at \\
\hline A_55_P2108837 & 1448232_x_at \\
\hline A_51_P392687 & 1456292_a_at \\
\hline A_55_P2004781 & 1416239_at \\
\hline
\end{tabular}



A_52_P679105
1437671_x_at
A_55_P2171116
1426808_at
A_52_P35048
1416168_at
A_52_P114905
1455239_at
A_51_P392687
1450641_at
A_51_P411271
1416543_at
A_51_P168613
1438684_at
A_55_P1978060
1423061_at
A_55_P2028015
1426910_at
A_66_P111562
1417420_at
A_51_P237752
1424130_a_at
A_55_P2105025
1455872_at
A_51_P321341
1427345_a_at
A_52_P426870
1438233_at
A_55_P2087607
1417496_at
1448276_at
1456312_x_at
A_55_P2013948
1416613_at
1422861_s_at
A_55_P2056241
1448475_at
A_55_P2213209
1423754_at
A_51_P128876
1434845_at 


\begin{tabular}{|c|c|c|}
\hline AGILENT Gene Symbol & AFFY Gene Symbol & q-value \\
\hline Akr1c14 & Akr1c14 & 0 \\
\hline Anxa1 & Anxa1 & 0 \\
\hline Ogn & Ogn & 0 \\
\hline Fbln5 & Fbln5 & 0 \\
\hline Spp1 & Spp1 & 0 \\
\hline Anxa3 & Anxa3 & 0 \\
\hline Fmod & Fmod & 0 \\
\hline Cfh & Cfh & 0 \\
\hline 1500015010Rik & 1500015010Rik & 0 \\
\hline Lox & Lox & 0 \\
\hline Shroom3 & Shroom3 & 0 \\
\hline Prss23 & Prss23 & 0 \\
\hline Hspb1 & Hspb1 & 0 \\
\hline $\mathrm{Cp}$ & $\mathrm{Cp}$ & 0 \\
\hline Ndrg1 & Ndrg1 & 0 \\
\hline Col8a1 & Col8a1 & 1.19E-04 \\
\hline Tfcp2I1 & Tfcp2l1 & 1.19E-04 \\
\hline Tagln & Tagln & 1.19E-04 \\
\hline Thbs1 & Pald1 | Thbs1 & 1.19E-04 \\
\hline Ndrg1 & Ndrg1 & 1.19E-04 \\
\hline Fmod & Fmod & 1.19E-04 \\
\hline $\mathrm{Cp}$ & $\mathrm{Cp}$ & 1.19E-04 \\
\hline Cfh & Cfh & 1.19E-04 \\
\hline Fmod & Fmod & 1.19E-04 \\
\hline Lgals1 & Lgals1 & 1.19E-04 \\
\hline Ifitm1 & Ifitm1 & 1.19E-04 \\
\hline Hspb1 & Hspb1 & 1.19E-04 \\
\hline Ccnd1 & Cond1 & $2.15 \mathrm{E}-04$ \\
\hline Klhdc8a & Klhdc8a & 2.15E-04 \\
\hline \multirow[t]{2}{*}{$\mathrm{Cp}$} & $\mathrm{Cp}$ & $2.15 \mathrm{E}-04$ \\
\hline & 0 Ugt1a1 | Ugt1a10 | Ugt1a2 | Ugt1a5 & 4.17E-04 \\
\hline $\operatorname{Tgm} 2$ & Tgm2 & 4.90E-04 \\
\hline Cnn2 & Cnn2 & 4.90E-04 \\
\hline Ndrg1 & Ndrg1 & $4.88 \mathrm{E}-04$ \\
\hline \multirow[t]{2}{*}{ Mmp2 } & Mmp2 & $4.88 \mathrm{E}-04$ \\
\hline & 0 S100a6 & $4.88 \mathrm{E}-04$ \\
\hline Crabp1 & Crabp1 & $4.88 \mathrm{E}-04$ \\
\hline Tgm2 & Tgm2 & 4.88E-04 \\
\hline Parp3 & Parp3 & $4.88 \mathrm{E}-04$ \\
\hline Folh1 & Folh1 & $4.88 \mathrm{E}-04$ \\
\hline Pdlim2 & Pdlim2 & $6.20 \mathrm{E}-04$ \\
\hline Tgm2 & $\operatorname{Tgm} 2$ & $6.20 \mathrm{E}-04$ \\
\hline Gpr126 & Gpr126 & $6.82 \mathrm{E}-04$ \\
\hline Mme & Mme & 7.41E-04 \\
\hline
\end{tabular}




\begin{tabular}{|c|c|c|}
\hline 1500015010Rik & 1500015010Rik & 8.70E-04 \\
\hline $\mathrm{Cp}$ & $\mathrm{Cp}$ & 0.001020408 \\
\hline Anxa2 & Anxa2 & 0.001020408 \\
\hline Tnfrsf12a & Tnfrsf12a & 0.001020408 \\
\hline Lgals1 & Lgals1 & 0.001069182 \\
\hline Col3a1 & Col3a1 & 0.001069182 \\
\hline Cond1 & Cond1 & 0.001069182 \\
\hline Parp3 & Parp3 & 0.001069182 \\
\hline Akap12 & Akap12 & 0.001358025 \\
\hline Tspo & Tspo & 0.001369048 \\
\hline Cd44 & Cd44 & 0.001369048 \\
\hline Ecm1 & Ecm1 & 0.001637427 \\
\hline Cdk1 & Cdk1 & 0.001724138 \\
\hline$A 2 m$ & $\mathrm{~A} 2 \mathrm{~m}$ & 0.001777778 \\
\hline Ifi35 & Ifi35 & 0.001777778 \\
\hline Fkbp5 & Fkbp5 & 0.001912568 \\
\hline Ahnak & Ahnak & 0.002239583 \\
\hline $\operatorname{lgf} 2 b p 2$ & Igf $2 b p 2$ & 0.002239583 \\
\hline Aurkb & Aurkb & 0.002239583 \\
\hline Crlf1 & Crlf1 & 0.002575758 \\
\hline Serping1 & Serping1 & 0.002575758 \\
\hline Ddo & Ddo & 0.00254902 \\
\hline Tspo & Tspo & 0.00254902 \\
\hline P4ha3 & P4ha3 & 0.002560386 \\
\hline Ndrg1 & Ndrg1 & 0.002571429 \\
\hline Cep55 & Cep55 & 0.002676056 \\
\hline \multirow[t]{2}{*}{ Pla2g4a } & Pla2g4a & 0.002685185 \\
\hline & 0 Cald1 & 0.002844445 \\
\hline Tnfrsf12a & Tnfrsf12a & 0.002844445 \\
\hline Pter & Pter & 0.002844445 \\
\hline Top2a & Top2a & 0.002850877 \\
\hline Col5a2 & Col5a2 & 0.00278481 \\
\hline Bmp6 & Bmp6 & 0.00278481 \\
\hline Glycam1 & Glycam1 & 0.00278481 \\
\hline Ctnnal1 & Ctnnal1 & 0.002705882 \\
\hline Ctgf & Ctgf & 0.002705882 \\
\hline Synpo2 & Synpo2 & 0.002705882 \\
\hline Cntf & Cntf | Zfp91 | Zfp91Cntf & 0.002705882 \\
\hline Tspo & Tspo & 0.002705882 \\
\hline Gfap & Gfap & 0.002705882 \\
\hline Matn2 & Matn2 & 0.002720307 \\
\hline Ephx1 & Ephx1 & 0.002720307 \\
\hline \multirow[t]{2}{*}{ Hspb8 } & Hspb8 & 0.002954545 \\
\hline & 0 Samd9l & 0.003183521 \\
\hline Tuba1c & Gm6682 | Tuba1c & 0.003333333 \\
\hline Vim & Vim & 0.003443223 \\
\hline Ass1 & Ass1 | Gm5424 & 0.003514493 \\
\hline
\end{tabular}




$\begin{array}{llr}\text { Prss23 } & \text { Prss23 } & 0.003942652 \\ \text { Lgals3 } & \text { Lgals3 } & 0.00393617 \\ \text { Serpinf1 } & \text { Serpinf1 } & 0.003964912 \\ \text { Ifitm10 } & \text { Ifitm10 } & 0.00395189 \\ \text { Vim } & \text { Vim } & 0.00395189 \\ \text { Nfe2I2 } & \text { Nfe2I2 } & 0.003973064 \\ \text { Nuak1 } & \text { Nuak1 } & 0.003973064 \\ \text { Arvcf } & \text { Arvcf } & 0.004125413 \\ \text { Pawr } & \text { Pawr } & 0.004125413 \\ \text { Ccnd1 } & \text { Ccnd1 } & 0.004150327 \\ \text { Ptrf } & \text { Ptrf } & 0.004174757 \\ \text { Fam167a } & \text { Fam167a } & 0.004285714 \\ \text { Sult1a1 } & \text { Sult1a1 } & 0.004285714 \\ \text { Fam178a } & \text { Fam178a } & 0.004299066 \\ \text { Cp } & \text { Cp } & 0.004299066 \\ \text { Tspan4 } & \text { Tspan4 } & 0.004567901 \\ \text { Gsn } & \text { Gsn } & 0.004617737 \\ \text { Cyp1b1 } & \text { Cyp1b1 } & 0.004761905 \\ \text { Pdlim5 } & \text { Pdlim5 } & 0.004761905 \\ \text { LOC269472 } & \text { Olfml3 } & 0.004761905 \\ \text { Ifitm3 } & \text { Ifitm3 } & 0.004778761 \\ & \text { OGm20204 | Gm20204 } & 1.1075892\end{array}$




\section{RP-value}

17.760506

21.299797

44.875797

45.48008

46.285477

46.47518

61.248623

69.88809

71.779144

76.01784

79.818085

82.06712

83.2879

86.75464

98.28696

101.41915

101.56774

104.86032

107.77535

109.78165

112.0289

118.08096

119.00356

119.12291

122.52708

123.0083

124.67552

133.05362

134.14148

138.51173

144.09425

155.89572

156.913

158.77759

161.34007

162.31116

163.87465

164.34166

164.54904

164.72581

168.93918

169.91945

174.94891

184.028 


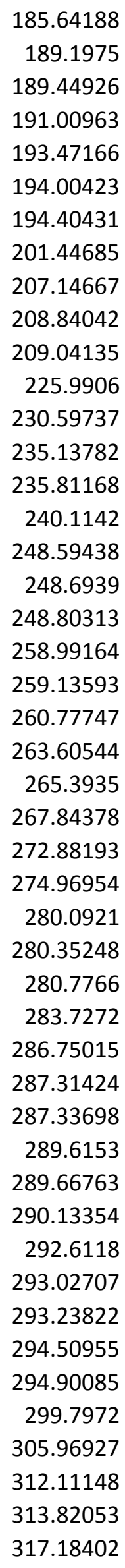




$$
\begin{array}{r}
326.07068 \\
327.4596 \\
329.2706 \\
330.53345 \\
332.79706 \\
333.51178 \\
333.60986 \\
337.1255 \\
337.49805 \\
339.68878 \\
343.50995 \\
344.82422 \\
345.2598 \\
347.7215 \\
347.7488 \\
352.6393 \\
354.39297 \\
356.665 \\
357.09262 \\
357.35934 \\
358.40176 \\
8950.983
\end{array}
$$


1

2

3

4

5

6

7

8

9

\begin{tabular}{|c|c|c|c|c|c|}
\hline ProbeName & GeneSymbol & GeneName & Description & $\log F C$ & AveExpr \\
\hline A_55_P2180869 & Ocel1 & occludin/ELL dor & m Mus musculus occlt & 1.912680866 & 11.20738139 \\
\hline A_66_P105689 & Trim34a & tripartite motif-c & cc Mus musculus tripa & 1.832444376 & 8.366008201 \\
\hline A_55_P2085142 & Spp1 & secreted phosph & c Mus musculus secre & f 1.726988441 & 9.069085973 \\
\hline A_55_P2130129 & Kcnab1 & potassium voltag & g๕ Mus musculus pota & 1.64885182 & 9.034037272 \\
\hline A_51_P451458 & Mamdc2 & MAM domain co & r Mus musculus MA^ & 1.503395436 & 9.872073547 \\
\hline A_52_P209484 & Tmem88 & transmembrane & F Mus musculus trans & 1.360071196 & 699972 \\
\hline A_55_P2137611 & Irgm2 & immunity-relate & d Mus musculus imm & 1.142352135 & 9.334410797 \\
\hline A_55_P2056325 & Anxa3 & annexin $\mathrm{A} 3$ & Mus musculus ann€ & $=1.066791$ & 34223 \\
\hline A_51_P237383 & Rnase4 & ribonuclease, $\mathrm{RN}$ & İ Mus musculus ribor & 1.058616352 & 10.71759463 \\
\hline A_66_P140976 & Rpl15 & ribosomal protei & ir Mus musculus ribos & 0.958862454 & 12.01103127 \\
\hline A_51_P140237 & Fhl2 & four and a half $L$ & ulus four & 0.944947122 & 12.347438 \\
\hline A_55_P2176248 & & 0 & BB001409 RIKEN fu & 0.789714112 & 15.19223654 \\
\hline A_52_P604629 & Csrnp1 & & i. Mus musculus cyst $\epsilon$ & 0.636119273 & 10.39213101 \\
\hline A_51_P149562 & Apbb2 & 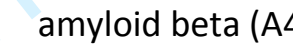 & 4) Mus musculus amyl & | 0.59661532 & 12.68664241 \\
\hline A_55_P2033445 & Tnfrsf1b & & a Mus musculus tume & 0.547667986 & 6.372954616 \\
\hline A_52_P563617 & Ssbp4 & single stranded [ & Mus musculus singl & 0.543415824 & 12.00448531 \\
\hline A_51_P124748 & Tgfb3 & transforming gro & Mus musculus trans & 0.54195087 & 14.82434995 \\
\hline A_55_P2006525 & Adamtsl4 & ADAMTS-like 4 & Mus musculus ADA & 0.52369464 & 11.96438562 \\
\hline A_52_P363216 & Gcnt2 & glucosaminyl ( $\mathrm{N}-$ & a Mus musculus gluce & 0.443700852 & 9.454501915 \\
\hline A_51_P411728 & 2900026A02Rik & RIKEN cDNA 290 & O Mus musculus RIKE & 0.412405535 & 6.947356535 \\
\hline A_55_P2383283 & 2310001H17Rik & RIKEN cDNA 231 & O Mus musculus adul 1 & 0.390172821 & 6.442100669 \\
\hline A_66_P107790 & Myl12a & myosin, light cha & i Mus musculus myo: & 0.385361306 & 16.30849845 \\
\hline A_51_P266774 & Mfn2 & mitofusin 2 & Mus musculus mito & 0.341591338 & 11.2941752 \\
\hline
\end{tabular}




\begin{tabular}{rrr}
\hline \multicolumn{1}{c}{ adj.P.Val } & Orre-Log_FC & Orre-adj.P.Val \\
\hline $6.74098 \mathrm{E}-11$ & 0.671147264 & 0.017244532 \\
$1.75238 \mathrm{E}-08$ & 1.040119507 & 0.010939297 \\
0.000151451 & 1.985300298 & 0.000905207 \\
0.000246485 & 2.159113265 & 0.016663479 \\
$8.60945 \mathrm{E}-07$ & 1.706249745 & 0.023407529 \\
$2.13081 \mathrm{E}-07$ & 1.300105703 & 0.012253122 \\
0.043028606 & 1.227755556 & 0.012427216 \\
$8.60945 \mathrm{E}-07$ & 1.853241477 & 0.000707922 \\
0.00045366 & 1.145738027 & 0.020785778 \\
$3.02697 \mathrm{E}-06$ & 0.916973791 & 0.007254029 \\
0.002411981 & 1.050243221 & 0.044629988 \\
$3.57358 \mathrm{E}-09$ & 0.527909643 & 0.035359423 \\
0.01141401 & 1.06350743 & 0.046781615 \\
0.042282655 & 1.17925114 & 0.002867076 \\
0.003725106 & 0.932289499 & 0.019878077 \\
0.001311367 & 0.597294453 & 0.014909633 \\
0.045134321 & 2.050401115 & 0.009907979 \\
0.034730892 & 1.060613269 & 0.023818682 \\
0.029590022 & 1.329891434 & 0.0203074 \\
0.046775339 & 0.856409089 & 0.040114262 \\
0.020221663 & 1.92503457 & 0.038211201 \\
0.004062968 & 1.460600323 & 0.000771054 \\
0.00320903 & 0.7318489 & 0.047061769
\end{tabular}




\begin{tabular}{|c|c|c|c|c|}
\hline ProbeName & GeneSymbol & Description & $\operatorname{logFC}$ & AveExpr \\
\hline _52_P267391 & Trim12a & tripartite motif-coı Mus musculus tripa & 5.326621277 & 7.971349421 \\
\hline 55_P1974432 & Gm5067 & predicted gene 50 । Mus musculus $13 \mathrm{~d}$ i & 4.706882168 & 7.941454285 \\
\hline _55_P2070576 & Ccl27a & hemokine ( $\mathrm{C}-\mathrm{C} \mathrm{m}$. Mus musculus chen & 3.159607921 & 8.528529626 \\
\hline _55_P2205650 & 2610507I01Rik & RIKEN cDNA 2610: Mus musculus RIKEI & 2.76634258 & 8.199204967 \\
\hline _55_P2064659 & Trim12a & tripartite motif-coı Mus musculus tripa & 2.625034338 & 7.006201591 \\
\hline _55_P2256646 & C130078N14 & uncharacterized pı Mus musculus $16 \mathrm{~d}$; & 2.57614419 & 6.975926163 \\
\hline _55_P2068731 & Gm20878 & predicted gene, $2 \mathrm{C}$ Mus musculus pred & 2.535424208 & 9.5022869 \\
\hline _52_P360330 & Map1b & microtubule-assoc Mus musculus micrı & 2.528377361 & \\
\hline 55_P2068733 & Gm20878 & predicted gene, 2 C Mus musculus pred & 2.4557783 & 8968 \\
\hline _55_P2092750 & Car9 & carbonic anhydras Mus musculus carbi & 2.227984321 & 46767 \\
\hline 55_P1981756 & Vmn2r123 & vomeronasal 2, rer Mus musculus vomı & 2.222558264 & \\
\hline _51_P349888 & Ang2 & angiogenin, ribonı Mus musculus angic & 2.221223649 & 7.45 \\
\hline _52_P88793 & Zfp933 & zinc finger protein Mus musculus zinc 1 & 2.187870963 & 202 \\
\hline _52_P238846 & Bpnt1 & bisphosphate 3'-nı Mus musculus bispr & 2.17951172 & 8.499105459 \\
\hline _51_P288549 & Jmjd7 & jumonji domain co Mus musculus jumc & 2.155696224 & 9.038758021 \\
\hline _66_P130730 & Zfp963 & zinc finger protein Mus musculus zinc 1 & 2.1427 & \\
\hline _55_P2112986 & Klk1k & kallikrein 1-relatec Mus musculus kallik & 2.141524133 & \\
\hline 55_P2134591 & BC049715 & cDNA sequence $B($ Mus musculus $c D N /$ & 2.123548403 & 7.11520769 \\
\hline _55_P2097151 & Ptchd2 & d domain cc Mus musculus patcl & 2.000 & 1801 \\
\hline -55_P2073965 & BC049715 & cDNA sequence $B($ Mus musculus $c D N /$ & 1.994500273 & 6.92 \\
\hline _51_P217498 & Slc2a4 & solute carrier fami Mus musculus solut & 1.966656603 & 8.57 \\
\hline 55_P2045886 & Stk3 & serine/threonine $\mathrm{k}$ Mus musculus serin & 1.9168 & 422 \\
\hline A_55_P2108773 & 4930427 & RIKEN cDNA 4930< Mus musculus RIKEI & 1.903303041 & 5089 \\
\hline A_66_P134474 & Ang3 & angiogenin, ribonı Mus musculus angic & 1.797 & 889 \\
\hline A_55_P1964628 & LOC102633627 & 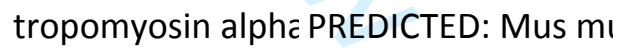 & 1.763 & \\
\hline _51_P293069 & Mfsd7b & musculus majo & 1.750158707 & 096 \\
\hline A_55_P2154387 & Bmp4 & torphogene Mus musculus bon€ & 1.725246422 & 9438 \\
\hline A_51_P413 & Klk1b3 & 1 & 1.701 & \\
\hline _51_P112627 & St6galr & ST6 (alpha-N-acet) Mus musculus ST6 ( & 1.692648894 & 4601 \\
\hline A_51_P512820 & Dera & 2-deoxyribose-5-p Mus musculus 2-de & 1.680832596 & 1435 \\
\hline _55_P2095603 & Ccdc65 & coil domain Mus musculus coile & 1.6808176 & 111 \\
\hline A_51_P477121 & Pmaip1 & phorbol-12-myrist: Mus musculus phor & 1.661805686 & 9.694988353 \\
\hline A_55_P2373852 & 2310058N22Rik & RIKEN cDNA 2310 C Mus musculus adult & 1.602415256 & 633 \\
\hline A_55_P2144597 & 9030025 & RIKEN cDNA 9030C Mus musculus RIKEI & 1.574018429 & 058 \\
\hline A_51_P180724 & Mlh1 & mutL homolog 1 (E Mus musculus mutl & 1.554543727 & 10.33206306 \\
\hline A_51_P142896 & Cd59a & CD59a antigen Mus musculus CD5؟ & 1.5382424 & 11.07205551 \\
\hline A_55_P2003561 & Luzp2 & leucine zipper prot Mus musculus leuci & 1.53117695 & 10.52421298 \\
\hline A_51_P417321 & Zfp236 & zinc finger protein Mus musculus zinc 1 & 1.517239691 & 7.995104458 \\
\hline A_51_P115953 & Ctxn3 & cortexin $3 \quad$ Mus musculus cort $\epsilon$ & 1.515844412 & 7.480665769 \\
\hline A_55_P2068723 & Ccl27a & chemokine ( $\mathrm{C}-\mathrm{C} \mathrm{m}$ ) Mus musculus chen & 1.506485285 & 9.878089363 \\
\hline A_55_P2068734 & Ccl27a & chemokine ( $\mathrm{C}-\mathrm{C} \mathrm{m}$ ) Mus musculus chen & 1.501278472 & 10.14705428 \\
\hline A_55_P2015912 & Zfp961 & zinc finger protein Mus musculus zinc 1 & 1.460709523 & 7.978713091 \\
\hline A_55_P1961395 & Pdpn & podoplanin $\quad$ Mus musculus podc & 1.408511896 & 15.4731878 \\
\hline A_51_P358722 & Lancl3 & LanC lantibiotic syı Mus musculus LanC & 1.403818102 & 7.624333535 \\
\hline A_55_P2053551 & Mast4 & microtubule assoc Mus musculus micrı & 1.383573947 & 9.459809864 \\
\hline
\end{tabular}


A_55_P2019577 1500011B03Rik

A_52_P532687 Ermard

A_52_P490207 Ermard

A_55_P2059357 Myo7a

A_51_P507899 Ttc8

A_55_P2194064 BC023969

A_55_P2067727 Mxra7

A_55_P2197338 Tnfsf13os

A_51_P345316 Cep76

A_55_P2065726 Snx29

A_55_P2179793

A_52_P642012

A_52_P616332

A_51_P179504

A_55_P2169963

A_52_P559545

A_55_P2180196

A_52_P135707

A_52_P598634

A_52_P587738

A_55_P2380428

A_66_P122158

A_52_P592909

A_52_P549977

A_51_P125368

A_55_P2019054

A_55_P1987196

A_55_P1960167

A_51_P480013

A_55_P2120866

A_51_P418908

A_55_P1975874

A_51_P286814

A_55_P2108883

A_55_P2083213

A_51_P490747

A_52_P311853

A_55_P2065059

A_55_P1998401

A_55_P2079158

A_55_P2153941

A_55_P1974602

A_51_P155174

A_55_P2097156

A_52_P295104

A_55_P2151138

A_55_P2201612
BC006965

Atp10d

Ang3

Gm13152

Cercam

Ccdc32

Creb3

1190007I07Rik

P2ry2

5430416G10Rik

Pisd-ps3

Dgat2

Fam32a

Hars

Acacb

Gm3448

Bcat2

Car11

Gm7120

Larp1

Bcl2I15

Ncor2

AV356131

Purb

Al593442

Ddit4l

Wnt2

Eif2ak4

Fam154b

Zfp386

Map2k7

Zfp672

Ptchd2

Smim5

Dynlt1f

Slc30a7
RIKEN cDNA 1500 ( Mus musculus RIKEI 1.382367568

ER membrane assc Mus musculus ER m 1.374998141

ER membrane assc Mus musculus ER m 1.288727894

myosin VIIA Mus musculus myo: 1.280346209

tetratricopeptide r Mus musculus tetra 1.276939334

cDNA sequence BC Mus musculus lung 1.267825845

matrix-remodellin؛ Mus musculus matr 1.262225358

tumor necrosis fac PREDICTED: Mus mI 1.258335257

centrosomal prote Mus musculus centı 1.257149573

sorting nexin 29 sorting nexin 29 [So 1.257094651

0

0 GAG_IPMAE (P3179

1.25413714

CDNA sequence BC Mus musculus CDN/ 1.25400917

ATPase, class V, ty Mus musculus ATPa 1.247972291

angiogenin, ribonı Mus musculus angic 1.243578938

predicted gene 13 Mus musculus pred 1.236720209

cerebral endotheli Mus musculus cerel 1.218979981

coiled-coil domain coiled-coil domain c 1.210677001

cAMP responsive $\epsilon$ Mus musculus CAM 1.208699556

RIKEN cDNA 1190 C Mus musculus RIKEI 1.196215216

purinergic recepto Mus musculus purir 1.195637072

RIKEN cDNA 5430<Mus musculus 6 da) 1.190354222

phosphatidylserint Mus musculus phos 1.175927068

diacylglycerol O-ac Mus musculus diacy 1.173956077

family with sequer Mus musculus famil 1.173692938

histidyl-tRNA syntl Mus musculus histic 1.172348692

acetyl-Coenzyme $/$ Mus musculus acet) 1.168117595

predicted gene 34. Mus musculus pred 1.167901495

branched chain an Mus musculus bran 1.149749383

carbonic anhydras Mus musculus carbr 1.147119938

predicted gene 71: Mus musculus pred 1.137640533

La ribonucleoprotє Mus musculus La rik 1.137191779

BCLI2-like $15 \quad$ Mus musculus BCLl; 1.121558908

nuclear receptor c Mus musculus nucle 1.109882524

expressed sequen، Mus musculus adult 1.104483644

purine rich elemer Mus musculus purir 1.07740577

expressed sequenı Mus musculus exprı 1.075390771

DNA-damage-indu Mus musculus DNA. 1.07360728

wingless-related N Mus musculus wing 1.071997776

eukaryotic translat eukaryotic translati 1.065720732

family with sequer Mus musculus famil 1.060527374

zinc finger protein Mus musculus zinc 11.059050992

mitogen-activated Mus musculus mito 1.042915394

zinc finger protein Mus musculus zinc 11.034852851

patched domain c( Mus musculus patcl 1.034050443

small integral men Mus musculus smal 1.026324687

dynein light chain 'Mus musculus dyne 1.011748282

solute carrier fami Mus musculus solut 1.003319035
11.13618528

9.208882095

8.746606738

7.864933543

10.06822585

7.04159718

11.32684755

7.508009275

7.422795425

7.59703769

11.05468172

7.4799979

7.18748402

7.705143143

6.482225328

8.680124663

6.693191129

11.79138848

10.74842945

10.79148861

6.615967993

9.074216706

10.39327982

11.4060602

11.44773653

8.899943125

9.058289041

9.932383882

10.42364956

9.945520617

9.48531631

6.994147168

14.09285663

10.05893668

10.71629864

7.060184729

11.17332195

6.654513312

7.579710465

7.097166998

10.08765784

7.206717795

9.47375292

6.365934281

7.028001519

15.31604468

6.695524338 
A_55_P2109544 Trnt1

A_66_P120770 Ywhaz

A_55_P1983268 4930444P10Rik

A_51_P191520 Stard10

A_66_P130366 Stk38l

A_55_P2293414 1700001C19Rik

A_55_P2018330 Gm13298

A_51_P470989 Paip1

A_55_P2127587 Smcr8

A_55_P1972490 Psg16

A_55_P1955483 Grb14

A_66_P123055 Gm10845

A_51_P275496 BC026762

A_52_P682745 Dock4

A_52_P20639 Rd3

A_51_P494863 Vmac

A_55_P2021094 Tmem181b-ps

A_55_P2149382 Gm3448

A_55_P2221647 Al605517

A_55_P1989524 Fndc1

A_51_P159293 Zbbx

A_55_P1995924 Gm13157

A_51_P452820 Rpl31

A_52_P123738 Rnf41

A_52_P222230

A_51_P327564 Glb1

A_51_P422335 Zfp420

A_55_P2146749 Rps13

A_51_P375558 Муос

A_51_P123604 Ppwd1

A_55_P1962756 TtIl2

A_51_P316801 Wdr60

A_51_P228193 Ociad1

A_55_P1998995 Speg

A_52_P489778 Ablim1

A_52_P512553 Atg1612

A_65_P08864 Dph5

A_55_P1985428 Atg1612

A_52_P325477 Trim16

A_66_P108434 Ccdc65

A_55_P1970033 Per1

A_51_P409893 Prkar2a

A_55_P2161465 Gm10516

A_55_P2031547 Vmn2r121

A_55_P2062133 Etv3

A_51_P314153 Nr2c2ap

A_51_P140607 Asun
tRNA nucleotidyl t Mus musculus tRNA 1.001272322 tyrosine 3-monoos Mus musculus tyros 0.997607559 RIKEN cDNA 4930< Mus musculus RIKEI 0.994940226 START domain con Mus musculus STAR 0.994448253 serine/threonine $k$ Mus musculus serin 0.993625574 RIKEN cDNA 1700C Mus musculus RIKEI 0.990165687 predicted gene 13: Mus musculus pred 0.986638051 polyadenylate binc Mus musculus poly: 0.972419001 Smith-Magenis syr Mus musculus Smitl 0.96794916 pregnancy specific PREDICTED: Mus mI 0.96746518 growth factor rece Mus musculus grow 0.961537973 predicted gene 10: Mus musculus pred 0.961441464 cDNA sequence BC Mus musculus cDN/ 0.956225426 dedicator of cytoki Mus musculus dedic 0.953245847 retinal degeneratic Mus musculus retin 0.952385121 vimentin-type inte Mus musculus vime 0.951203788 transmembrane pı Mus musculus pred 0.950888888 predicted gene 34. Mus musculus pred 0.949503743 expressed sequenc Mus musculus adult 0.946454699 fibronectin type III Mus musculus fibro 0.939270273 zinc finger, B-box c Mus musculus zinc 10.938960672 predicted gene 13 Mus musculus pred 0.936224377 ribosomal protein Mus musculus ribos 0.935299927 ring finger protein Mus musculus ring 10.934464172 0

0 Mus musculus adult 0.932941732 galactosidase, bet: Mus musculus galac 0.930537406 zinc finger protein Mus musculus zinc 10.926862021 ribosomal protein Mus musculus ribos 0.926468304 myocilin Mus musculus myor 0.91987638 peptidylprolyl ison Mus musculus pept 0.918453225 tubulin tyrosine lig Mus musculus tubu 0.915979939 WD repeat domair Mus musculus WD r 0.91300515 OCIA domain cont: Mus musculus OCIA 0.902626422 SPEG complex locı Mus musculus SPEC 0.890537687 actin-binding LIM | Mus musculus actin 0.888374228 autophagy related Mus musculus auto 0.884566919 DPH5 homolog (S. Mus musculus 4 da) 0.882305084 autophagy related Mus musculus auto 0.878149304 tripartite motif-coı Mus musculus tripa 0.875770007 coiled-coil domain Mus musculus coile $\quad 0.8752307$ period circadian $\mathrm{cl}$ Mus musculus peric $\quad 0.87102614$ protein kinase, cAIMus musculus prott 0.870456096 predicted gene 10: Mus musculus pred 0.869660084 vomeronasal 2, rer Mus musculus vomı 0.868193142 ets variant gene 3 Mus musculus ets v 0.865016273 nuclear receptor 2 Mus musculus nucle 0.857212287 asunder, spermatc Mus musculus asun 0.854600958
8.431184682

9.774694003

9.194778744

10.48259345

7.328617183

7.960315863

6.426310477

10.13248007

6.547066031

12.66758381

10.67699007

12.63602144

10.39653181

10.42480206

6.56148348

9.571912151

7.161708914

8.596002824

7.987944032

9.499426291

7.015945354

8.820762873

9.628451691

9.579431688

10.09808608

12.02546985

7.796718242

15.57163982

7.037334081

7.857252107

6.474892893

9.671748455

12.05023919

9.842623979

10.72166376

10.25934981

6.622110984

11.34750968

7.500056636

6.761612792

11.30179325

8.073370053

8.602021347

6.340248683

8.229462351

10.25241127

11.24260127 
A_55_P2012694 Kcnh5

A_52_P253179 Igfbp3

A_55_P1968977 Stk38I

A_55_P1982454 Eps8

A_55_P2000007 LOC102639358

A_51_P481821 Spcs3

A_51_P480202 Dlx2

A_55_P2062688 Msi1

A_55_P2023697 Zfp386

A_51_P320022 Atp10a

A_51_P356579 Mxra7

A_55_P1967514 Dnah7a

A_52_P217710 Fzd6

A_55_P2036723 Stk36

A_55_P1978226 Park2

A_55_P1999958

A_66_P112301

A_51_P244824

A_55_P1969431

A_55_P2145521

A_55_P2259125

A_55_P2206605

A_55_P2151143

A_51_P333965

A_52_P456561

A_55_P2183735

A_52_P565279

A_51_P448391

A_55_P1985693

A_51_P269634

A_55_P2033480

A_55_P2170509

A_51_P143468

A_52_P436590

A_52_P547589

A_55_P1990134

A_55_P2002226

A_66_P101108

A_55_P1987151

A_55_P1988882

A_55_P2144280

A_55_P2255737

A_55_P2045114

A_55_P2186558

A_55_P2212498

A_55_P2067652

A_55_P1995992

\section{C230072F16Rik}

Dapp1

Lyrm5

Stk38l

D7Ertd143e

5830444B04Rik

Dynlt1c

Cisd2

Abcd1

Pisd

Cecr5

Nkiras1

Fhdc1

Zfp14

Gm13298

Yipf4

KIhl26

Wbscr17

Spag1

Cox18

Dzip1

Tnrc18

Nlrp5

Sept9

Nnt

Tmem242

Tmem 242

C030005K06Rik

Boc

Gm14432 potassium voltage Mus musculus pota: 0.853264496 insulin-like growth Mus musculus insul 0.843238604 serine/threonine k Mus musculus serin 0.839143396 epidermal growth Mus musculus epidı 0.83854701 uncharacterized L( PREDICTED: Mus mı 0.837915964 signal peptidase cc Mus musculus signa 0.834205312 distal-less homeok Mus musculus dista 0.831967305 musashi RNA-bind Mus musculus musi 0.824555268 zinc finger protein Mus musculus zinc 10.824299376 ATPase, class V, ty Mus musculus ATPa 0.823382272 matrix-remodellinı Mus musculus matr 0.821065949 dynein, axonemal, Mus musculus dyne 0.816310076 frizzled homolog 6 Mus musculus frizzl 0.813851943 serine/threonine $k$ Mus musculus serin 0.812564111 Parkinson disease Mus musculus Parki 0.809691701 0

0 Mus musculus 2 cel 0.805965228 RIKEN cDNA C230( PREDICTED: Mus mI 0.80311273 dual adaptor for $p$ l Mus musculus dual 0.802564631 LYR motif containil Mus musculus LYR r 0.79913581 serine/threonine k Mus musculus serin 0.798266378 DNA segment, Chr Mus musculus DNA 0.796972973 RIKEN cDNA 5830 <Mus musculus RIKEI 0.79643716 dynein light chain Mus musculus dyne 0.795550998 CDGSH iron sulfur Mus musculus CDG! 0.792549929 ATP-binding casset Mus musculus ATP- 0.789316322 phosphatidylserinє Mus musculus phos 0.785035418 cat eye syndrome Mus musculus cat e 0.784649746 NFKB inhibitor int $\epsilon$ Mus musculus NFKE 0.783418591 FH2 domain conta Mus musculus FH2 10.782106906 zinc finger protein Mus musculus zinc 10.776997444 predicted gene 13: Mus musculus pred 0.774332816 Yip1 domain famil Mus musculus Yip1 0.772816822 kelch-like $26 \quad$ Mus musculus kelct 0.77198704 Williams-Beuren s'Mus musculus Willi; 0.769558866 sperm associated : Mus musculus sperı 0.769269498 cytochrome c oxid Mus musculus cytor 0.76750367 DAZ interacting pr. Mus musculus DAZ 0.767356331 trinucleotide repei Mus musculus trinu 0.76600373 NLR family, pyrin c Mus musculus NLR 0.762876701 septin $9 \quad$ Mus musculus septi 0.760689134 nicotinamide nucle Mus musculus nicot 0.755399056 0 0 Mus musculus $16 \mathrm{di} \quad 0.751739655$ transmembrane pı Mus musculus trans 0.746315876 transmembrane pı Mus musculus trans 0.745602274 RIKEN cDNA C030( Mus musculus 16 di 0.744696963 biregional cell adh Mus musculus bireg 0.744606754 predicted gene 14، Mus musculus pred 0.734175842
8.804906186 11.69049314

9.521077412

8.848665964

8.84202077

11.87147738

8.505777767

7.664091488

11.04881999

9.570566289

7.115904662

7.747422009

8.487905687

6.839913205

9.930960787

9.252652714

7.553472865

8.572185508

11.07024261

10.21390203

7.136638653

6.400644648

14.87675664

8.447908953

8.448857429

11.98425589

7.912325413

9.121554978

11.3884465

8.491684404

6.342741632

11.90136956

10.29776949

12.28938237

8.242367136

10.11076173

6.538813239

11.76838989

7.174930312

13.17396197

6.408132733

7.646039652

12.40287643

11.74115813

7.496432326

10.68001093

10.19189597 
A_51_P263246 Dusp8

A_51_P433091 Purb

A_52_P106620 Tnfrsf11b

A_51_P324934 Mcm3

A_51_P193302 Mrps7

A_51_P342707 Pold2

A_55_P2163729 Tvp23a

A_55_P2085333 Fbxo44

A_55_P1967820 Al661453

A_55_P1980292 Purb

A_55_P1964638 Cxadr

A_55_P1956918 Adamts5

A_55_P2037883 Ino80

A_55_P2173927 Insr

A_51_P487913 2600006K01Rik

A_55_P2028370

A_55_P1991851

A_55_P2144285

A_55_P1955568

A_55_P1976993

A_55_P2140118

A_52_P260696

A_55_P2154943

A_52_P627068

A_55_P2211937

A_55_P2045658

A_55_P2322029

A_55_P1979929

A_52_P646979

A_55_P2146500

A_51_P127915

A_51_P383629

A_52_P67200

A_55_P2026818

A_55_P1995874

A_55_P2122633

A_66_P110742

A_51_P414548

A_55_P2035717

A_55_P1974088

A_55_P2044982

A_55_P1974855

A_52_P14526

A_52_P484838

A_55_P2161695

A_51_P102507

A_55_P2000798
Speg

Nnt

Ext12

Strn

Qpct

Arnt2

LOC102633020

Disp2

E130101M22

Nme6

3830406C13Rik

Prcp

D16Ertd472e

Ccdc107

Rnasek

Vps4a

Stt3b

Slc4a7

Gm14326

Airn

Casp7

Pgap2

Stard6

Zfp74

Zfp868

Zyg11b

Rfxank

Kdelc1

Vps33a

Ccdc144b dual specificity phr Mus musculus dual 0.729339237

purine rich elemer Mus musculus purir 0.729313164

tumor necrosis fac Mus musculus tumc 0.724063196

minichromosome | Mus musculus 2 da) 0.723982874

mitchondrial ribos Mus musculus mitcl 0.719882585

polymerase (DNA I Mus musculus polyr 0.718347678

trans-golgi networ Mus musculus trans 0.714329056

F-box protein 44 Mus musculus F-bo: 0.711686096

expressed sequenc Mus musculus exprı 0.707301965

purine rich elemer Mus musculus purir $\quad 0.70306024$

coxsackie virus anc Mus musculus coxsi 0.702794142

a disintegrin-like a Mus musculus a disi 0.701754973

INO80 homolog (S Mus musculus INO8 0.693705043 insulin receptor Mus musculus insul 0.691688622 RIKEN CDNA 2600 (Mus musculus 10 di 0.69118068

0 BC009829 Sin3A ass 0.690461183

SPEG complex locı Mus musculus SPEC 0.687259254 nicotinamide nucle Mus musculus nicot 0.68724313 exostoses (multipl. Mus musculus exos 0.685268426 striatin, calmoduliı Mus musculus strial 0.680102547 glutaminyl-peptid€ Mus musculus gluta 0.678864447 aryl hydrocarbon r Mus musculus aryl \& 0.677915652 uncharacterized L( PREDICTED: Mus mı 0.676814658 dispatched homolı Mus musculus dispe 0.674397108 uncharacterized pı Mus musculus 0 da) 0.671839525 NME/NM23 nucler Mus musculus NME 0.671023993 RIKEN CDNA 3830 LPREDICTED: Mus mI 0.66786877 prolylcarboxypept Mus musculus proly 0.66627182 DNA segment, Chr Mus musculus DNA 0.664518727 coiled-coil domain coiled-coil domain c 0.664242439 ribonuclease, RNa: Mus musculus ribor 0.660400658 vacuolar protein si Mus musculus vacu 0.659040627 STT3, subunit of th Mus musculus STT3 0.65786142 solute carrier fami Mus musculus solut 0.65742112 predicted gene 14: Mus musculus pred 0.655155313 antisense Igf2r RN. Mus musculus 12 di 0.655073738 0

0 predicted gene 994! 0.652494644 caspase $7 \quad$ Mus musculus caspi 0.652297056 post-GPI attachme Mus musculus post- 0.649830054 StAR-related lipid I Mus musculus StAR 0.644654813 zinc finger protein Mus musculus zinc 10.643862306 zinc finger protein Mus musculus zinc 10.642298042 zyg-Il family memk Mus musculus zyg-I $\quad 0.6399025$ regulatory factor $X$ Mus musculus regul 0.638156992 KDEL (Lys-Asp-Glu. Mus musculus KDEL 0.633604449 vacuolar protein sı Mus musculus vacu 0.632936888 coiled-coil domain Mus musculus coile 0.630687382
8.550859775 14.36585406

9.178956716 11.08620242 10.07382699 10.00505646 10.78639596 8.802084472 7.567482964 12.08650609 10.81708985 8.144140925 7.913768321 7.865164283 7.997964919 6.864620982 9.934616638 12.56592392 11.19675553 7.201136783 8.702572561 11.15522419 6.813185302 7.74997714 7.244348901 9.369172174 6.852701833 7.089942414 8.526185962 9.312354149 11.60556053 8.892115274 12.59516264 11.80618119 9.473693824 9.447841546 6.927579723 9.262799375 11.01902213 7.252685872 8.623261586 9.44786431 8.984744753 10.24113363 8.732323513 10.47605776 8.797090317 
A_55_P2049095 Atat1

A_51_P312748 Oxsm

A_55_P2035667 Gyk

A_55_P2048483 D17Zt10e

A_66_P105736 Net1

A_52_P244463 D16Ertd472e

A_51_P487027 Kcnk2

A_51_P233027 Rmdn3

A_51_P310676 Galr2

A_51_P119031 Naa30

A_55_P2133220 Arhgef39

A_55_P2028522 Stac

A_51_P253897 Psca

A_51_P261560 Tex33

A_51_P186798

A_55_P2030383 Frmd4a

A_55_P2098578 Tubd1

A_51_P181722 Rbks

A_51_P280532 Supt16

A_55_P2118891 Zfp882

A_55_P1989341 Ntng1

A_52_P644534 Dhrsx

A_66_P138585 4833419G08Rik

A_55_P1998947 Trim16

A_51_P234544 Azin1

A_52_P1187949 Trim5

A_51_P367780 Adamtsl2

A_55_P2146996 Wdr52

A_55_P2173073 Zfp931

A_51_P283292 Gm14326

A_52_P376360 Pdgfc

A_55_P1954436 Gm7967

A_55_P1988424 Tpi1

A_65_P02321 Usp36

A_51_P343851 Tgfbrap1

A_55_P1967538 Hunk

A_51_P246727 Mlxip

A_55_P2187043 Tpm1

A_51_P454103 Manba

A_52_P494514 Insr

A_55_P2057994 Mif4gd

A_55_P2007243 Kcnc1

A_51_P441494 BC100451

A_52_P74576 Ccdc65

A_55_P1993876 Otud7a

A_51_P382928 Cstf3

A_52_P259558 Ogfod1

alpha tubulin acet! Mus musculus alphi $0.630435448 \quad 9.141766982$ 3-oxoacyl-ACP syn Mus musculus 3-oxi $0.629777447 \quad 9.01476485$ glycerol kinase Mus musculus glyce $0.628787466 \quad 8.648364662$ DNA segment, Chr Mus musculus D17N 0.6287779626 .992196756 neuroepithelial cel Mus musculus neur $\quad 0.6286504 \quad 8.454326837$ DNA segment, Chr Mus musculus DNA 0.6284068529 .597217161 potassium channe Mus musculus pota: 0.6261681749 .661418919 regulator of micro Mus musculus regul $0.625932519 \quad 8.988246549$ galanin receptor 2 Mus musculus galar 0.6227244327 .162505651 $\mathrm{N}$ (alpha)-acetyltra Mus musculus N(alk $0.621554593 \quad 9.624039204$ Rho guanine nucle Mus musculus Rho | $0.616734048 \quad 8.642150922$ src homology thre Mus musculus src h $0.616010766 \quad 7.041364257$ prostate stem cell Mus musculus prost $0.615881617 \quad 6.42562448$ testis expressed 3: Mus musculus testi؛ 0.6153863397 .329209425

0

0 Mus musculus adult 0.61322874

FERM domain con Mus musculus FERN 0.612959998 tubulin, delta 1 Mus musculus tubu 0.612758828 ribokinase Mus musculus ribok 0.61159276 suppressor of Ty 1 Mus musculus supp 0.61157395 zinc finger protein Mus musculus zinc 10.610913984 netrin G1 Mus musculus netri 0.60958472 dehydrogenase/re Mus musculus dehy 0.609560651 RIKEN CDNA 4833/Mus musculus 0 da) 0.607587363 tripartite motif-col Mus musculus tripa 0.604488449 antizyme inhibitor Mus musculus antiz 0.602800226 tripartite motif-col tripartite motif-con 0.599764892 ADAMTS-like 2 Mus musculus ADAI 0.599729357 WD repeat domair Mus musculus WD r 0.597251838 zinc finger protein Mus musculus zinc +0.595265308 predicted gene 14: Mus musculus pred 0.595082261 platelet-derived gr Mus musculus plate 0.594601426 predicted gene 79I PREDICTED: Mus mI 0.594041539 triosephosphate is Mus musculus triosı 0.593343466 ubiquitin specific $k$ Mus musculus ubiq 0.591697254 transforming grow Mus musculus trans 0.589821522 hormonally upregı Mus musculus horr 0.588246584 MLX interacting pr Mus musculus MLX 0.588227683 tropomyosin 1, alk Mus musculus tropc 0.585164286 mannosidase, bete Mus musculus manı 0.584351312 insulin receptor Mus musculus insul 0.583352015 MIF4G domain cor Mus musculus MIF4 0.5807349 potassium voltage Mus musculus pota: 0.578759804 CDNA sequence BC Mus musculus CDN 0.576549426 coiled-coil domain Mus musculus coile 0.575074749 OTU domain conte Mus musculus OTU 0.56755514 cleavage stimulati Mus musculus cleav 0.567207944 2-oxoglutarate anc Mus musculus 2-oxı 0.564501633
9.016423032

10.90964601

8.954822571 9.245800439

8.530965999 8.294079552

8.476360633

9.25671223

6.595782617

7.155449916

11.96811488

6.187168827

7.341050691

7.020264708

8.866986993

9.015347234

10.96578443

9.155739992

12.72558299

7.853283573

7.554407441 11.69706941

8.368515877 15.06169489 9.614489926

8.423305987

11.5472407

9.232521563

7.490033464

7.18473457

6.807269488

11.55214483

8.350975048 
A_55_P2037081

A_52_P412529

A_51_P403636

A_55_P2062469

A_55_P1992715

A_55_P2113356

A_55_P2060278

A_55_P2039556

A_51_P308029

A_55_P2034600

A_55_P2241204

A_66_P113662

A_55_P1985554

A_55_P2148641

A_55_P1996086

A_55_P1966958

A_52_P144794

A_55_P1969166

A_55_P1973447

A_55_P2052563

A_55_P2078213

A_52_P110068

A_55_P2147791

A_55_P2026109

A_55_P2354336

A_66_P125212

A_55_P2076927

A_52_P558382

A_55_P2030721

A_52_P505143

A_51_P144648

A_55_P2147457

A_55_P2013396

A_55_P2172566

A_52_P137691

A_55_P2187038

A_52_P662098

A_51_P315555

A_55_P2144075

A_65_P17492

A_51_P213099

A_55_P2212259

A_51_P149621

A_55_P2047962

A_55_P1966863

A_52_P6828

A_55_P2102998
2610305D13Rik

Fbxo3

Smad7

Col12a1

Igfbp3

Miip

Fam45a

Pak6

2010107G23Rik

Gm5523

1500015A07Rik

Tmem62

B4galt4

Rab4a

Gm14325

Mef2d

Tceanc2

Fxr2

Ybx2

Id1

1700020I14Rik

Rqcd1

Fam129c

Rpap1

Mapk1ip1l

Ints10

Mapk8

Ankle1

Jrk

Vps13b

Gm6410

Gm14305

Insr

Trappc5

Tpm1

Net1

Nars

Pofut2

Med29

Ntng1

4930539J05Rik

Stt3b

Gjc2

$\operatorname{Mad} 212$

$\mathrm{Xk}$

Gm3893
RIKEN cDNA 2610ミ Mus musculus RIKEI 0.564272206

F-box protein 3 Mus musculus F-bo: 0.56094732

SMAD family mem Mus musculus SMA 0.558336877

collagen, type XII, : Mus musculus colla: 0.555681636 insulin-like growth insulin-like growth $f 0.554260468$ migration and inva Mus musculus migri 0.552280288 family with sequer Mus musculus famil 0.549155306 p21 protein (Cdc4; Mus musculus p21। 0.547684608 RIKEN cDNA 20101 Mus musculus RIKEI 0.54748727 glyceraldehyde-3-ן Mus musculus pred 0.546447402 RIKEN cDNA 1500 C Mus musculus RIKEI 0.54219336 transmembrane pı Mus musculus trans 0.538267077 UDP-Gal:betaGlcN Mus musculus UDP. 0.537628446 RAB4A, member R Mus musculus RAB $\angle 0.537243189$ predicted gene 14: Mus musculus pred 0.537186059 myocyte enhancer Mus musculus myor 0.536620471 transcription elon€ Mus musculus trans 0.53455295 fragile X mental re Mus musculus fragil 0.531489988 Y box protein $2 \quad$ Y box protein 2 [Sol 0.531057305 inhibitor of DNA bi Mus musculus inhib 0.53033599 RIKEN cDNA 1700 (Mus musculus 11 di 0.530002687 rcd1 (required for Mus musculus rcd1 0.529942113 family with sequer Mus musculus famil 0.529236236 RNA polymerase II Mus musculus RNA 0.527509786 0

0 Mus musculus activ 0.526987573 mitogen-activated Mus musculus mito 0.526302456 integrator comple: Mus musculus integ 0.525810261 mitogen-activated Mus musculus mito 0.525327826 ankyrin repeat anc Mus musculus anky 0.525007257 jerky Mus musculus jerky 0.522539156 vacuolar protein sı Mus musculus vacu 0.519678366 predicted gene 64 PREDICTED: Mus mI 0.519481968 predicted gene 14: Mus musculus pred 0.517683154 insulin receptor Mus musculus insul 0.515697068 trafficking protein Mus musculus traffi 0.513420389 tropomyosin 1, alf Mus musculus tropc 0.51307192 neuroepithelial cel Mus musculus neur 0.512228233 asparaginyl-tRNA $\subseteq$ Mus musculus aspa 0.509016823 protein O-fucosyltı Mus musculus protє 0.506738417 mediator complex Mus musculus medi 0.506204122 netrin G1 Mus musculus 0 da) 0.504996564 RIKEN cDNA 4930: Mus musculus RIKEI 0.504221596 STT3, subunit of th Mus musculus STT3 0.50217731 gap junction prote Mus musculus gap j 0.501911712 MAD2 mitotic arre Mus musculus MAD 0.497055694 Kell blood group p Mus musculus Kell $k \quad 0.49681471$ predicted gene 38! Mus musculus pred $\quad 0.496695$
6.91795213

9.33493547

8.78753881

11.31206825

7.33220213

8.129275828

10.93484853

6.8612788

9.996108859

9.212421977

8.26476772

9.893821557

10.83785546

9.172580706

9.111250799

7.75838435

7.249346109

12.34194972

7.109701064

13.47147372

6.877639522

8.065464761

7.217970946

11.77046652

8.20102921

11.42316344

10.3795043

6.850921359

7.995435084

8.542489357

6.7346537

6.166119882

7.864612391

7.323745759

9.062810254

15.71376893

6.892396013

10.21166179

9.580876216

11.08044267

9.917510525

6.774963856

10.53780671

8.075503933

12.22692429

6.75218636

6.443091727 
A_55_P2090152

A_55_P2041095 Six4

A_51_P282179 Mtor

A_51_P397375 Pet112

A_55_P1953226 Gm14484

A_55_P2017140 Vmn2r88

A_55_P2121344 Nudcd3

A_52_P85765 Stard6

A_55_P2161958 2410141K09Rik

A_55_P2167160 Hdhd2

A_55_P2023818 Cysltr1

A_55_P2051666 Nfkbib

A_55_P2154049 Myo18a

A_55_P2121038 Gm10825

A_52_P180826 Ndufaf7

A_55_P2155479 Eps8

A_55_P2169775 Ano3

A_55_P2341950 Crebzf

A_55_P2108808 Tubgcp4

A_55_P2021099 Tmem181a

A_52_P329314

A_55_P2015715 Rab4a

A_55_P2266880 Kirrel

A_51_P215190 Efcab11

A_51_P517381 Cers2

A_55_P2134645 Fam227a

A_55_P2115995 Sms

A_51_P211341 Eif3j1

A_55_P1998194 Snrpn

A_52_P65494 lqgap2

A_52_P404895 Tmem62

A_55_P2042146 Fech

A_55_P2081398

A_51_P221132 L2hgdh

A_55_P1974442 Sumf2

A_55_P2105563 1700104L18Rik

A_51_P257684 Stau1

A_52_P412452 Cntn6

A_55_P2187171 Sv2c

A_55_P1961645 Vcp

A_55_P1955172 Camk2d

A_55_P2433218 Bloc1s6

A_51_P292368 Tmco6

A_55_P2168118 4933406C10Rik

A_55_P2094881 Fgfr2

A_55_P2326337 BC026513

A_66_P111216 4632427E13Rik

vomeronasal 2, rer Mus musculus vomı 0.4965499986 .426258453 sine oculis-related Mus musculus sine $\quad 0.496515097 \quad 8.936492814$ mechanistic target Mus musculus mect $0.49615176 \quad 9.600335943$ PET112 homolog (! Mus musculus PET1 $0.491822478 \quad 10.63703531$ predicted gene 14. Mus musculus pred 0.4909685656 .890348424 vomeronasal 2, rer Mus musculus vomı 0.4899585846 .596218494 NudC domain cont Mus musculus Nud( 0.48661331214 .48644209 StAR-related lipid I Mus musculus StAR $0.485532131 \quad 7.245921317$ RIKEN cDNA 24101 Mus musculus RIKEI 0.4838972018 .944749485 haloacid dehaloge Mus musculus halo: 0.4836362468 .721553593 cysteinyl leukotrie Mus musculus cyst $\epsilon \quad 0.4822001226 .913667873$ nuclear factor of $k$ Mus musculus nucle 0.4818251096 .924766802 myosin XVIIIA myosin XVIIIA [Sour $0.481029403 \quad 9.351451737$ predicted gene 10: Mus musculus pred 0.4804946156 .490948216 NADH dehydrogen Mus musculus NAD 0.47819733212 .06995123 epidermal growth Mus musculus epidı 0.4777783916 .329612776 anoctamin $3 \quad$ PREDICTED: Mus mI 0.4774574856 .133871034 CREB/ATF bZIP tra Mus musculus CREE $0.477145148 \quad 8.496257562$ tubulin, gamma co Mus musculus tubu 0.4764591959 .030731709 transmembrane pı Mus musculus trans $0.475629474 \quad 11.52617749$

0

0 Mus musculus activ 0.474168018

RAB4A, member R Mus musculus RAB $\angle 0.472820882$ kin of IRRE like (Dr Mus musculus kin o 0.469979146 EF-hand calcium b Mus musculus EF-hi 0.468088991 ceramide synthas $\in$ Mus musculus cerar 0.467385174 family with sequer family with sequenc 0.466809009 spermine synthas $€$ Mus musculus sperı 0.46670616 eukaryotic transla1 Mus musculus euka 0.465694432 small nuclear ribor Mus musculus smal 0.465682748 IQ motif containin: Mus musculus IQ m 0.465013328 transmembrane pı Mus musculus trans 0.463251515 ferrochelatase Mus musculus ferro 0.458340686 0 0 Mus musculus 3 da) 0.457982439 L-2-hydroxyglutarc Mus musculus L-2-h 0.457098176 sulfatase modifyin Mus musculus sulfa 0.456851178 RIKEN CDNA 17001 Mus musculus RIKEI 0.454910321 staufen (RNA bind Mus musculus stauf 0.453790437 contactin $6 \quad$ Mus musculus conti 0.452227075 synaptic vesicle gl) Mus musculus syna| 0.451273743 valosin containing Mus musculus valos 0.44903777 calcium/calmoduli Mus musculus 13 di 0.447763023 biogenesis of lysos Mus musculus bioge 0.44500213 transmembrane ar Mus musculus trans 0.444879683 RIKEN cDNA 4933/Mus musculus RIKEI 0.444090327 fibroblast growth † Mus musculus fibro 0.440891409 cDNA sequence $B$ C Mus musculus CDN/ 0.440689436 RIKEN cDNA $4632 \angle$ Mus musculus 13 di 0.438953046
8.776230772

11.89110055

11.97410603

6.530560013

11.93577959

6.952928892 10.36200694

10.78643735

9.972197283

7.800658437

7.426336173

12.87623246

6.874884315

9.207769539

10.3700016 6.389015679

10.17502422

6.28573148 6.044621352 15.30971961 7.272524621 11.46295795 8.843225584 6.470058176 9.97808478 6.983094672 9.873997921 
A_55_P1962174 Rab8a

A_55_P2018681 G630016G05Rik

A_52_P417990 Zfp868

A_51_P125183 Coq5

A_55_P2261772 Lzts1

A_55_P2163363 Clec2f

A_55_P1998392 Eif2ak4

A_55_P2098305 Coq5

A_55_P2032478 Sfxn5

A_55_P2092776 Apoo

A_55_P2012241 Ctso

A_55_P2452384 Mga

A_52_P663526 Nmrk1

A_55_P1974063 Gm2545

A_55_P2050592 Gm5785

A_55_P1970578 Aamdc

A_52_P299358 Lclat1

A_55_P2288285 2310007J06Rik

A_55_P2115968 Gm14325

A_51_P336391 Tmem18

A_52_P67983 Lcmt2

A_51_P114456 Tceanc2

A_55_P1962384 Churc1

A_55_P2053206 Kdm4c

A_55_P1979246 Cep192

A_51_P341010 Ercc8

A_55_P2388687 1700003G18Rik

A_55_P2043430

A_55_P2078675

A_55_P2174273

A_52_P147666

A_55_P2085731

A_55_P2160737

A_55_P2170911

A_52_P425510

A_55_P2104532

A_52_P600946

A_55_P1999883

A_52_P916539

A_55_P2045535

A_55_P1973896

A_55_P2012107

A_52_P151905

A_55_P2169909

A_55_P2186460

A_51_P381230

A_55_P2161485

\section{Slco5a1}

Slc30a7

Stk38

Gm10366

Dnah7a

Acacb

Ccdc88c

Gm14499

Zbtb34

Rsg1

Vmn2r86

Vmn2r10

Gm5132

Ostm1

Emx2os

Zhx2

Ptchd2

RAB8A, member R Mus musculus RAB\& 0.424137876 RIKEN cDNA G6301 PREDICTED: Mus mI 0.42271889 zinc finger protein Mus musculus zinc 10.42043698 coenzyme Q5 hom Mus musculus coen 0.419476387 leucine zipper, put leucine zipper, puta 0.419198928 C-type lectin doma Mus musculus C-tyk 0.41913221 eukaryotic translat eukaryotic translatiı 0.417164393 coenzyme Q5 hom Mus musculus coen 0.416936983 sideroflexin $5 \quad$ sideroflexin 5 [Sourı 0.416637414 apolipoprotein O Mus musculus apoli 0.415844374 cathepsin O Mus musculus cathe 0.41559322 MAX gene associat Mus musculus MAX 0.415243264 nicotinamide ribos Mus musculus nicot 0.414414455 predicted gene 25، Mus musculus 2 da) 0.413708974 predicted gene 57: PREDICTED: Mus mı 0.413684181 adipogenesis assor Mus musculus adipr 0.411471092 Iysocardiolipin acy Mus musculus lysoc 0.409296709 RIKEN cDNA 2310 (Mus musculus adult 0.408617418 predicted gene 14: Mus musculus pred 0.406099667 transmembrane pı Mus musculus trans 0.40553698 leucine carboxyl m Mus musculus leuci 0.405340924 transcription elonģ Mus musculus trans 0.404316557 churchill domain c Mus musculus churı 0.40263635 Iysine (K)-specific ( Mus musculus Iysinı 0.402091708 centrosomal prote Mus musculus adult 0.401581925 excision repaiross- Mus musculus excis 0.398522876 RIKEN cDNA 1700 C Mus musculus 10 di 0.397935551

0

0

0 immunoglobulin ka| 0.397025619

0 Q8BSH3_MOUSE (C 0.394629866

solute carrier orga Mus musculus solut 0.394162375 solute carrier fami Mus musculus solut 0.392889528 serine/threonine k Mus musculus serin 0.392477146 0

0 T cell receptor alph; 0.392156901 predicted gene 10: Mus musculus RCB- 0.391173241 dynein, axonemal, Mus musculus dyne 0.39117049 acetyl-Coenzyme / Mus musculus acet) 0.390395955 coiled-coil domain Mus musculus coile 0.389765394 predicted gene 14، Mus musculus pred 0.387386265 zinc finger and BTE Mus musculus zinc $1 \quad 0.38576947$ REM2 and RAB-likt REM2 and RAB-like 0.383686004 vomeronasal 2, rer Mus musculus vomı 0.381511625 vomeronasal 2, reı Mus musculus vomı 0.379830315 predicted gene 51: Mus musculus pred 0.379563561 osteopetrosis asso osteopetrosis assoc 0.378501009 Emx2 opposite stri Mus musculus Emx; 0.376192058 zinc fingers and hc Mus musculus zinc 10.375666934 patched domain c(Mus musculus patcl 0.372827738
10.59066735

6.292352942

6.57986675

8.945056584

7.577093736

6.343168511

7.420248711

8.769634807

7.721938883

11.68965048

7.125120729

8.932685792

8.003857616

6.218528632

8.461838113

11.92703754

9.949768884

6.306220274

10.10361514

12.25146122

10.26010917

8.001395018

13.35054757

10.49093052

6.726663181

9.191087781

6.495699396

6.260679891

10.55175122

6.254228174

8.867834981

7.244947197

6.228406796

6.982595294

6.474571593

6.37202715

6.700200388

6.58660119

7.042352643

6.568754731

6.091046043

6.27031141

6.283761675

6.99491057

6.496326483

6.692151172

6.5520809 
A_55_P2160910 Faim2

A_55_P1986208 Ccnb1ip1

A_52_P622694 Adal

A_55_P2293967 Tbc1d5

A_55_P1953984 Gm11033

A_66_P130911 Proser2

A_52_P88818 Clybl

A_55_P2033481 Gm13298

A_66_P122377 Cd84

A_51_P320357 Grin2b

A_52_P504330 Ankib1

A_51_P394154 Ddx51

A_55_P2055642 2810407A14Rik

A_52_P219913 Cdan1

A_51_P220150 Angptl7

A_51_P228865 Zfp72

A_55_P1993094 Mesdc1

A_55_P2005853 Nacc2

A_55_P2057247 Etohi1

A_51_P226962 Capn15

A_51_P343429 Slc25a37

A_55_P2025363 Ccnl2

A_55_P2002918 Klc2

A_55_P1977649 Hoxd8

A_55_P2031382 Crebzf

A_55_P2124016 Nipsnap1

A_55_P2057268 Magi1

A_55_P2139713 Phf13

A_65_P10433 Rpp14

A_55_P2005680 Ipo11

A_55_P2134351 Lancl3

A_66_P122053 Kcnq5

A_55_P2002220 Dzip1

A_55_P1956687 Rab37

A_52_P221588 Gm5382

A_55_P2062787 Mfap3

A_55_P2094484 Gm14137

A_52_P18765 Hsbp1l1

A_55_P1983999 Desi1

A_51_P495581 Tlr1

A_55_P2197134 A930018M24Rik

A_52_P146403 Arhgef38

A_55_P2066219 Gm3455

A_51_P390775 Ube2e1

A_51_P494006 Scaf8

A_51_P243134 Adcy6

A_55_P1955851 Al593442
Fas apoptotic inhik Mus musculus Fas a 0.3715931156 .831524973

cyclin B1 interactir Mus musculus cyclit 0.3697102916 .103756239

adenosine deamin Mus musculus aden 0.3658722397 .522421143

TBC1 domain fami Mus musculus TBC1 0.3652519889 .774149604

predicted gene 11। Mus musculus lung 0.363501227 .161944693

proline and serine Mus musculus 15 di 0.3622762816 .964030088

citrate lyase beta I Mus musculus citrał 0.3619062659 .542409873

predicted gene 13: Mus musculus pred 0.3611372326 .680187468

CD84 antigen Mus musculus CD8< 0.3605062026 .583811787

glutamate recepto Mus musculus gluta $0.360280168 \quad 6.359031467$

ankyrin repeat anc Mus musculus anky $0.360250064 \quad 11.36207192$

DEAD (Asp-Glu-Ala Mus musculus DEA[ $0.359877757 \quad 10.16927723$

RIKEN cDNA 2810 L PREDICTED: Mus mI 0.3589511566 .040017011

congenital dyseryt Mus musculus cong $0.358850754 \quad 8.473046024$

angiopoietin-like 7 Mus musculus angic $0.357228462 \quad 6.165392545$

zinc finger protein Mus musculus zinc 10.3553003427 .847411318

mesoderm develo| Mus musculus mesc $0.350713908 \quad 6.76590339$

nucleus accumben Mus musculus nucle $0.348924514 \quad 15.54803706$

ethanol induced 1 Mus musculus etha| $0.348267114 \quad 7.063109429$

calpain $15 \quad$ Mus musculus calpc $0.344518115 \quad 6.57454886$

solute carrier fami Mus musculus solut $0.337178716 \quad 10.60161225$

cyclin L2 Mus musculus cyclit 0.3344045899 .599327514

kinesin light chain Mus musculus kines 0.32810048411 .33603232

homeobox D8 Mus musculus hom 0.3280270826 .093584714

CREB/ATF bZIP tra Mus musculus CREE 0.32752939810 .64772186

4-nitrophenylphos Mus musculus 4-nit $0.327066316 \quad 11.36759244$

membrane associa Mus musculus mem $0.32681088 \quad 12.32067837$

PHD finger protein Mus musculus PHD 0.3258652917 .367699171

ribonuclease P 14 : Mus musculus ribor 0.3212847249 .956294104

importin $11 \quad$ Mus musculus impc $0.319029262 \quad 12.22021613$

LanC lantibiotic syı Mus musculus LanC 0.3189403726 .245579756

potassium voltage Mus musculus pota: 0.3126250936 .374769691

DAZ interacting pri Mus musculus mRN 0.3104980767 .108950154

RAB37, member R. Mus musculus RAB $\equiv 0.3086904056 .946824503$

predicted gene 53: Mus musculus pred $0.30857958 \quad 6.411918635$

microfibrillar-assoı Mus musculus micrı $0.308502076 \quad 11.29521395$

predicted gene 14: Mus musculus pred $0.307841674 \quad 6.249040867$

heat shock factor I Mus musculus heat $0.305411046 \quad 6.175418302$

desumoylating iso| Mus musculus desu 0.3050509219 .223225353

toll-like receptor 1 Mus musculus toll-li $0.304755981 \quad 6.483885608$

RIKEN cDNA A930(Mus musculus adult $0.303146333 \quad 6.14613498$

Rho guanine nucle Mus musculus Rho। 0.2977136316 .022117917

predicted gene 34! Mus musculus adult $0.292306122 \quad 6.846252621$

ubiquitin-conjugat Mus musculus ubiq। $0.288261272 \quad 12.4664347$

SR-related CTD-ass Mus musculus SR-r€ $0.287834928 \quad 11.40734343$

adenylate cyclase I Mus musculus aden $0.286563466 \quad 10.60793854$

expressed sequenı Mus musculus exprı $0.285949357 \quad 6.264288781$ 
Page 149 of 190

Current Alzheimer Research

$\begin{array}{lll}1 & & \\ 2 & \text { A_55_P2024555 } & \text { Ppap2a } \\ 3 & \text { A_55_P2108868 } & \text { Nufip1 } \\ 4 & \text { A_51_P375693 } & \text { Tmem135 } \\ 5 & \text { A_55_P1982494 } & \text { 1700012L04Rik } \\ 6 & \text { A_51_P129100 } & \text { Sec63 } \\ 7 & \text { A_55_P2003228 } & \text { Rerg } \\ 8 & \text { A_55_P2060722 } & \text { Uso1 } \\ 9 & \text { A_55_P2052210 } & \text { Gdi2 } \\ 10 & \text { A_55_P1970887 } & \text { Vmn2r16 } \\ 11 & \text { A_65_P10180 } & \text { Rad23b } \\ 12 & \text { A_55_P2049483 } & \\ 13 & \text { A_52_P328078 } & \text { Atp5b } \\ 14 & \text { A_55_P2217548 } & \text { 4921515G04Rik } \\ 15 & \text { A_51_P263290 } & \text { Galnt9 } \\ 16 & \text { A_55_P2078815 } & \text { Xk } \\ 17 & \text { A_55_P2107447 } & \text { Rab37 } \\ 18 & \text { A_51_P150608 } & \text { Jagn1 } \\ 19 & \text { A_52_P580582 } & \text { Nppa } \\ 20 & & \\ 21 & & \end{array}$

phosphatidic acid | Mus musculus phos $0.28340742 \quad 12.70854687$

nuclear fragile X m Mus musculus nucle $0.28340483 \quad 8.406819665$

transmembrane pı Mus musculus trans $0.282838866 \quad 10.08696021$

RIKEN cDNA 1700C Mus musculus RIKEI 0.2803945256 .499018685

SEC63-like (S. cere Mus musculus SEC6 $0.279371698 \quad 9.600818144$

RAS-like, estrogen Mus musculus RAS- 0.2781747356 .186459568

USO1 vesicle docki Mus musculus USO: 0.2738644111 .79810942

guanosine diphosk Mus musculus guan $0.268127974 \quad 15.07008493$

vomeronasal 2, reı Mus musculus vomı 0.2679198665 .980558817

RAD23b homolog I Mus musculus RAD: 0.26650263411 .43165245

$\begin{array}{llll}0 & 0 \\ 0 & \text { UI-M-GIO-cek-h-17-I } 0.265694877 & 5.93789424\end{array}$

ATP synthase, $\mathrm{H}+\mathrm{t}$ Mus musculus ATP : 0.2625647916 .25543189

RIKEN cDNA 4921: Mus musculus adult $0.261423553 \quad 6.071820881$

UDP-N-acetyl-alph Mus musculus UDP. $0.260345438 \quad 6.151726529$

Kell blood group p Mus musculus Kell $k 0.254480206 \quad 6.21155289$

RAB37, member R. Mus musculus RAB 0.2441402917 .029616615

jagunal homolog 1 Mus musculus jagur $0.2368731 \quad 13.19987516$

natriuretic peptid€ Mus musculus natri $0.227610422 \quad 6.588381243$

https://mc04.manuscriptcentral.com/cares 


\begin{tabular}{rrr}
\hline adj.P.Val & Orre-Log_FC & Orre-adj.P.Val \\
\hline $1.5155 \mathrm{E}-21$ & 0.494015964 & 0.101276807 \\
$4.18232 \mathrm{E}-23$ & -0.208741888 & 0.86222962 \\
$2.51609 \mathrm{E}-15$ & 0.509514034 & 0.173245869 \\
$1.89631 \mathrm{E}-15$ & -0.721426483 & 0.168073428 \\
$6.07388 \mathrm{E}-14$ & 0.706101378 & 0.198794714 \\
$4.55163 \mathrm{E}-12$ & -0.645492101 & 0.561180497 \\
$5.66539 \mathrm{E}-10$ & 0.382869744 & 0.300412533 \\
$2.68489 \mathrm{E}-12$ & 1.530274252 & 0.10751908 \\
$5.03003 \mathrm{E}-15$ & 0.607561951 & 0.180640491 \\
0.011383754 & 0.708072732 & 0.095841743 \\
$6.99163 \mathrm{E}-09$ & 0.417157621 & 0.732697156 \\
$1.00373 \mathrm{E}-08$ & 0.348358531 & 0.722818593 \\
$1.76297 \mathrm{E}-14$ & -0.461955973 & 0.69554264 \\
$7.34401 \mathrm{E}-09$ & -0.265301426 & 0.85895072 \\
$9.52423 \mathrm{E}-12$ & 0.132054376 & 0.806731065 \\
$2.51609 \mathrm{E}-15$ & -0.319929586 & 0.487450339 \\
$1.26452 \mathrm{E}-13$ & -0.30277199 & 0.776862477 \\
$3.81067 \mathrm{E}-16$ & 0.423154735 & 0.751201622 \\
$1.71376 \mathrm{E}-13$ & -0.511838677 & 0.522961414 \\
$1.32742 \mathrm{E}-15$ & -0.104540487 & 0.937077956 \\
$8.45076 \mathrm{E}-09$ & 1.128015324 & 0.251670799 \\
$6.39585 \mathrm{E}-07$ & 0.393027073 & 0.583894548 \\
$5.0348 \mathrm{E}-13$ & -0.042822396 & 0.97312979 \\
$1.87675 \mathrm{E}-06$ & -0.077991108 & 0.910601338 \\
$1.62674 \mathrm{E}-08$ & 0.700674909 & 0.157310536 \\
$9.79076 \mathrm{E}-06$ & -0.346039523 & 0.750752793 \\
0.002006738 & 0.363396889 & 0.739710744 \\
$1.20027 \mathrm{E}-10$ & -0.321280956 & 0.739926932 \\
$9.90908 \mathrm{E}-05$ & 1.089900036 & 0.110643562 \\
$1.34408 \mathrm{E}-05$ & -0.328214025 & 0.839521089 \\
$1.10036 \mathrm{E}-06$ & 0.437062716 & 0.598329105 \\
0.00193586 & 0.689481662 & 0.092676384 \\
$1.9256 \mathrm{E}-09$ & 0.861615264 & 0.251034963 \\
$4.0172 \mathrm{E}-06$ & -0.363493756 & 0.486203135 \\
$1.77247 \mathrm{E}-14$ & -0.184267324 & 0.609527127 \\
$3.00086 \mathrm{E}-06$ & 1.268073293 & 0.059985475 \\
0.002874148 & -1.61715276 & 0.065441134 \\
$1.94892 \mathrm{E}-09$ & -0.181696617 & 0.81968488 \\
0.000172194 & -0.157933401 & 0.719679153 \\
0.000877619 & -0.128163665 & 0.838921279 \\
0.001129742 & -0.086616262 & 0.918491027 \\
\hline $.92927 \mathrm{E}-08$ & -0.489734962 & 0.595972008 \\
$3.00086 \mathrm{E}-06$ & -1.135394748 & 0.284746979 \\
\hline $.62656 \mathrm{E}-06$ & 1.171930897 & 0.186324197 \\
\hline
\end{tabular}




\begin{tabular}{|c|c|c|}
\hline 1.13328E-08 & -0.113275734 & 0.894025902 \\
\hline 7.54693E-07 & -0.227365388 & 0.707333422 \\
\hline 3.00779E-07 & -0.088017319 & 0.938485136 \\
\hline 0.000151278 & -0.100899014 & 0.849011492 \\
\hline $3.2388 \mathrm{E}-06$ & -0.349722197 & 0.719898927 \\
\hline $6.28876 \mathrm{E}-05$ & -0.664151751 & 0.338510783 \\
\hline 0.001641709 & 0.742797052 & 0.080935733 \\
\hline 4.49879E-06 & -0.135059276 & 0.882935769 \\
\hline 1.0619E-08 & -0.169947384 & 0.892526632 \\
\hline $1.65847 \mathrm{E}-12$ & -0.307697854 & 0.641328681 \\
\hline $3.32214 \mathrm{E}-07$ & -0.424691967 & 0.444760948 \\
\hline 0.001489104 & -1.157946462 & 0.330185942 \\
\hline 8.45947E-07 & 0.845414186 & 0.365791074 \\
\hline 0.000101522 & 0.707672554 & 0.168664396 \\
\hline 3.57358E-09 & 0.553731131 & 0.647569617 \\
\hline $1.34579 E-06$ & -0.320991989 & 0.516380365 \\
\hline 2.60497E-08 & 0.213144118 & 0.725963984 \\
\hline 1.9256E-09 & -0.204806123 & 0.573717182 \\
\hline 5.21515E-09 & -0.481573263 & 0.072468507 \\
\hline 0.000396451 & -0.127132138 & 0.878705558 \\
\hline 1.14267E-07 & -0.845345713 & 0.302189147 \\
\hline 0.011911037 & -0.436640002 & 0.425322426 \\
\hline $6.61822 \mathrm{E}-08$ & 0.12927286 & 0.907153397 \\
\hline $2.33153 \mathrm{E}-08$ & 0.28379144 & 0.580809949 \\
\hline 0.002800997 & -0.145749407 & 0.784052969 \\
\hline $4.13642 \mathrm{E}-05$ & 0.226120011 & 0.69554264 \\
\hline 0.000407261 & 0.086866091 & 0.887775569 \\
\hline 0.001686638 & -0.63980779 & 0.082399826 \\
\hline 0.000808174 & -0.121826605 & 0.820857056 \\
\hline 8.79673E-09 & -0.536393529 & 0.698553736 \\
\hline 0.000189232 & -0.175484885 & 0.809427246 \\
\hline $2.63238 \mathrm{E}-05$ & -0.013684635 & 0.992257293 \\
\hline $6.28876 \mathrm{E}-05$ & 0.029962335 & 0.948802911 \\
\hline 1.11737E-05 & 0.22137264 & 0.824760267 \\
\hline 0.016323043 & 0.470246434 & 0.601138648 \\
\hline $2.84266 \mathrm{E}-07$ & -0.067080822 & 0.964389247 \\
\hline 9.61673E-06 & -0.339238132 & 0.790694488 \\
\hline 5.55739E-06 & 0.059781564 & 0.975871464 \\
\hline $1.9168 \mathrm{E}-05$ & -0.013439338 & 0.990510763 \\
\hline 0.000427139 & 0.447834803 & 0.738308088 \\
\hline 1.43E-09 & 0.000976591 & 0.999393864 \\
\hline $2.43926 \mathrm{E}-06$ & -0.680573205 & 0.1180523 \\
\hline 9.88697E-06 & -0.519690316 & 0.070583758 \\
\hline $2.75871 \mathrm{E}-10$ & -1.035950628 & 0.39089491 \\
\hline 0.013270961 & 1.792970376 & 0.152119333 \\
\hline 8.16187E-07 & 0.536623925 & 0.067094894 \\
\hline $1.0803 \mathrm{E}-06$ & -0.402527074 & 0.714474121 \\
\hline
\end{tabular}




\begin{tabular}{|c|c|c|}
\hline 9.10813E-08 & -0.0884533 & 0.944831525 \\
\hline 0.017215445 & 0.214479384 & 0.728038356 \\
\hline 0.000426641 & -0.350065867 & 0.568731758 \\
\hline 0.000396451 & 0.350261399 & 0.700220974 \\
\hline 5.39858E-08 & -0.000770162 & 0.999311806 \\
\hline 0.002746233 & 0.518897284 & 0.531876039 \\
\hline 5.32555E-10 & -0.523888849 & 0.455688565 \\
\hline 2.66649E-05 & -0.082641617 & 0.901318559 \\
\hline 2.33153E-08 & -0.067664713 & 0.944831525 \\
\hline 0.003856341 & -0.311484797 & 0.644089282 \\
\hline 0.032023049 & 0.801679878 & 0.272374934 \\
\hline $9.25444 \mathrm{E}-05$ & -0.533474707 & 0.197890713 \\
\hline 0.000580121 & -0.694080695 & 0.335852093 \\
\hline 0.000245655 & -0.445709032 & 0.40001535 \\
\hline $6.56904 \mathrm{E}-05$ & 0.090970165 & 0.946152468 \\
\hline $6.41213 \mathrm{E}-05$ & 0.145174467 & 0.855871263 \\
\hline 0.000200769 & -0.46867821 & 0.647058366 \\
\hline 0.000226702 & 0.122331107 & 0.860951268 \\
\hline 0.004185517 & 1.215372159 & 0.133330557 \\
\hline 0.037876068 & -0.289080588 & 0.654741331 \\
\hline 0.00732291 & 0.255868136 & 0.796533306 \\
\hline 9.48239E-05 & 0.485161194 & 0.724124183 \\
\hline 0.009804102 & 0.271313713 & 0.438743872 \\
\hline $7.88421 \mathrm{E}-07$ & -0.359228447 & 0.540486499 \\
\hline 0.000228528 & 0.1005591 & 0.923754161 \\
\hline 2.70552E-05 & -0.479348533 & 0.066856402 \\
\hline $9.56544 \mathrm{E}-07$ & -0.473266278 & 0.656861723 \\
\hline $1.46332 \mathrm{E}-08$ & 0.419511615 & 0.06877729 \\
\hline 0.000484742 & 0.461133994 & 0.435461001 \\
\hline 0.000186626 & -0.189919105 & 0.979780693 \\
\hline 4.55977E-07 & -0.628082537 & 0.577504928 \\
\hline $2.54921 \mathrm{E}-07$ & -0.154880818 & 0.887577752 \\
\hline 0.001153761 & 0.045572013 & 0.937286143 \\
\hline 0.000797911 & 0.822685256 & 0.164250851 \\
\hline $6.2652 E-05$ & 0.854454407 & 0.210081543 \\
\hline 4.05156E-05 & 0.18406571 & 0.853191719 \\
\hline $7.2128 \mathrm{E}-07$ & -0.614345578 & 0.612173152 \\
\hline 0.000826698 & 0.040459997 & 0.965681273 \\
\hline 2.04915E-05 & 0.744022505 & 0.486270386 \\
\hline 2.04688E-05 & 1.107747447 & 0.293728889 \\
\hline 1.12404E-07 & -0.636971337 & 0.158299143 \\
\hline 0.001247848 & 0.834773853 & 0.220903957 \\
\hline 0.013650381 & -0.309420255 & 0.840838909 \\
\hline 7.09229E-05 & 0.303300143 & 0.828466698 \\
\hline $1.20561 \mathrm{E}-05$ & 0.457626773 & 0.377351703 \\
\hline 0.008704796 & -0.291424239 & 0.243290114 \\
\hline 3.24393E-06 & -0.245466726 & 0.736884352 \\
\hline
\end{tabular}




\begin{tabular}{|c|c|c|}
\hline 0.001553895 & -0.2390392 & 0.775066835 \\
\hline 4.12901E-05 & 0.934771118 & 0.233602249 \\
\hline 0.000798312 & 0.22319446 & 0.796455726 \\
\hline 0.022055148 & -0.917940314 & 0.133641052 \\
\hline 7.47489E-09 & -0.694356039 & 0.078477358 \\
\hline 0.005950991 & 0.222865538 & 0.713806716 \\
\hline 0.00411945 & -0.509013023 & 0.465995005 \\
\hline $6.18358 \mathrm{E}-05$ & -0.927245633 & 0.240098905 \\
\hline $3.29145 E-05$ & -0.153864655 & 0.882847667 \\
\hline 0.002079095 & 1.35097666 & 0.085173746 \\
\hline 6.61661E-05 & 0.565810581 & 0.482680368 \\
\hline 0.001299081 & -0.177746544 & 0.80242013 \\
\hline 0.009131232 & 1.15840701 & 0.083102374 \\
\hline $6.44035 \mathrm{E}-05$ & -0.167372128 & 0.817273275 \\
\hline 0.003737457 & -0.909960332 & 0.066944948 \\
\hline 0.013677795 & -0.257058331 & 0.807223941 \\
\hline 0.04676798 & 0.484827214 & 0.571087362 \\
\hline 0.007172418 & -0.613699324 & 0.232972235 \\
\hline 0.002578168 & -0.072342826 & 0.93189109 \\
\hline 0.003231136 & 0.094367677 & 0.880089213 \\
\hline 0.000182659 & -0.201062051 & 0.785580413 \\
\hline $5.14628 \mathrm{E}-07$ & -0.072300221 & 0.964362185 \\
\hline $8.79832 E-05$ & 0.269129185 & 0.306411953 \\
\hline 0.00131247 & -0.152166942 & 0.887775569 \\
\hline 0.034321854 & 0.047372976 & 0.944629072 \\
\hline 0.001106059 & -0.657205133 & 0.107121807 \\
\hline 0.001416146 & -0.916051136 & 0.303085671 \\
\hline 7.31445E-05 & -0.283027394 & 0.739465708 \\
\hline 0.000125368 & -0.86592828 & 0.303518048 \\
\hline 3.99813E-05 & -0.410576823 & 0.656382082 \\
\hline $4.47682 \mathrm{E}-06$ & -0.554958189 & 0.621006089 \\
\hline 0.002072259 & -0.021108935 & 0.977261173 \\
\hline $2.04915 \mathrm{E}-05$ & 0.033525302 & 0.975783078 \\
\hline 0.016104221 & 1.821419607 & 0.189486201 \\
\hline 0.037863432 & -0.547783208 & 0.529143074 \\
\hline 0.000243506 & -0.026415405 & 0.97801984 \\
\hline 3.64274E-05 & 0.077953171 & 0.967021702 \\
\hline 0.002490508 & 0.087207661 & 0.887681593 \\
\hline 0.00047951 & 0.655819425 & 0.306656823 \\
\hline 0.013601838 & -0.344164461 & 0.410612403 \\
\hline 9.57333E-05 & -0.406650886 & 0.65354995 \\
\hline 0.009041276 & 0.478964704 & 0.418875176 \\
\hline 0.000988354 & 0.430537155 & 0.141438755 \\
\hline 0.00070165 & 0.378128234 & 0.342897773 \\
\hline 0.002292724 & -0.766273403 & 0.288269591 \\
\hline 0.007946676 & -0.534980075 & 0.41006599 \\
\hline 0.000374165 & -0.34410392 & 0.835063179 \\
\hline
\end{tabular}




\begin{tabular}{|c|c|c|}
\hline 0.003487058 & 0.780210233 & 0.254192071 \\
\hline 0.009131232 & 0.321292713 & 0.635367869 \\
\hline 0.013552166 & 0.558429729 & 0.388103578 \\
\hline 0.001686638 & 0.32439975 & 0.336941261 \\
\hline 0.001437493 & -0.083828839 & 0.877278291 \\
\hline 0.000345837 & -0.000894789 & 0.999107759 \\
\hline 0.000679021 & -0.475438804 & 0.569349756 \\
\hline 0.001450271 & -1.093896936 & 0.063107309 \\
\hline 0.024168112 & 0.955367737 & 0.183856842 \\
\hline 0.007247914 & 0.163323672 & 0.801843562 \\
\hline 0.007124108 & 0.721428476 & 0.343167684 \\
\hline 0.000380742 & 1.019376532 & 0.283366476 \\
\hline 0.00041466 & -0.047047455 & 0.967305665 \\
\hline 5.83498E-05 & 0.049656902 & 0.966150288 \\
\hline 0.019807083 & -0.279990455 & 0.713080554 \\
\hline 0.000159695 & -0.132772037 & 0.916677914 \\
\hline 5.66966E-06 & 0.435967444 & 0.206028172 \\
\hline 0.001867808 & -0.015299325 & 0.982726396 \\
\hline 0.000304942 & -0.474992494 & 0.323144086 \\
\hline 2.44643E-05 & 0.060060723 & 0.965395371 \\
\hline 0.002995138 & 1.123802351 & 0.058030957 \\
\hline 0.015404752 & -0.891573262 & 0.066989223 \\
\hline 0.0004735 & -0.329687077 & 0.669004414 \\
\hline 0.006661276 & -0.199950193 & 0.821033175 \\
\hline 0.029648475 & -0.877640248 & 0.31770382 \\
\hline 0.000394056 & -0.291408661 & 0.461045722 \\
\hline 1.66026E-05 & 0.295933448 & 0.80352741 \\
\hline 0.001266613 & 0.322045332 & 0.689215673 \\
\hline 0.001081614 & 0.452102103 & 0.529614566 \\
\hline 0.0158393 & -0.324175339 & 0.707027795 \\
\hline 0.038259174 & 0.247975874 & 0.32585137 \\
\hline 0.000415434 & 0.05079392 & 0.974584058 \\
\hline 0.003478829 & -0.663674202 & 0.162323347 \\
\hline 0.022577663 & 0.265453786 & 0.649672614 \\
\hline 3.99168E-05 & -0.736401868 & 0.471473845 \\
\hline 0.028405759 & 1.054215428 & 0.186012227 \\
\hline 0.000430633 & 0.28692132 & 0.780315128 \\
\hline 0.000452844 & 0.518092073 & 0.186366127 \\
\hline 0.013658962 & -0.49518021 & 0.230379161 \\
\hline 0.001443363 & 0.358380188 & 0.784426947 \\
\hline 3.20959E-05 & 0.004751345 & 0.997529424 \\
\hline 0.004087672 & -0.335252168 & 0.522632048 \\
\hline 0.020743492 & -0.447086891 & 0.49049781 \\
\hline 1.29096E-05 & -0.209494913 & 0.482680368 \\
\hline 0.020376763 & -0.218976164 & 0.865562087 \\
\hline 8.88541E-06 & 0.056266654 & 0.865562087 \\
\hline 0.002995138 & -0.846060792 & 0.173571483 \\
\hline
\end{tabular}




\begin{tabular}{|c|c|c|}
\hline 0.015496846 & -0.215456949 & 0.805001544 \\
\hline 0.008859233 & -0.448682911 & 0.485931171 \\
\hline 0.020857286 & -0.927601622 & 0.294121955 \\
\hline 0.000454218 & -0.382266557 & 0.369181579 \\
\hline 0.001325376 & 1.550632462 & 0.226406862 \\
\hline 0.024375049 & -0.036989877 & 0.979021192 \\
\hline 0.028521196 & -0.477180893 & 0.546591892 \\
\hline 0.00041681 & 0.007261906 & 0.994595766 \\
\hline 0.025433517 & -0.093872308 & 0.929526296 \\
\hline $3.24669 \mathrm{E}-05$ & -0.010881313 & 0.971976742 \\
\hline 0.006713462 & 0.669881453 & 0.430902741 \\
\hline 0.004203953 & 1.363007003 & 0.241387475 \\
\hline 0.001828957 & 0.155422066 & 0.806004571 \\
\hline 0.000430633 & -0.427253092 & 0.526950381 \\
\hline 0.00010589 & -0.705852549 & 0.473978686 \\
\hline 0.012561396 & -0.634432983 & 0.153566319 \\
\hline 0.000116895 & -0.272014313 & 0.803968048 \\
\hline 0.000509744 & -0.152951946 & 0.681379676 \\
\hline 0.006150735 & 0.297291172 & 0.768133169 \\
\hline 0.002925808 & -0.240046812 & 0.846394604 \\
\hline 0.041927762 & 0.307554775 & 0.816896693 \\
\hline 0.005506386 & 0.222849563 & 0.569392931 \\
\hline 0.000169155 & -0.246304142 & 0.820181198 \\
\hline 0.000475896 & 0.686029806 & 0.620916602 \\
\hline 7.35276E-06 & -0.27653707 & 0.630500872 \\
\hline 0.007377634 & 0.493585051 & 0.80444631 \\
\hline 0.031503546 & 1.068265984 & 0.073982416 \\
\hline 0.010923347 & 0.449900989 & 0.567548087 \\
\hline 0.024143297 & -0.216322332 & 0.909818319 \\
\hline 0.004317506 & -0.348045294 & 0.822944058 \\
\hline 0.011374659 & 0.626042573 & 0.273568523 \\
\hline 0.003849801 & -0.765261452 & 0.363636322 \\
\hline 0.00664579 & -0.301494635 & 0.402215667 \\
\hline 0.000436557 & -0.218451418 & 0.718797153 \\
\hline 0.000156036 & -0.048167815 & 0.961241863 \\
\hline 7.9413E-05 & -0.950805388 & 0.195702861 \\
\hline 0.037863432 & -0.018757085 & 0.987634819 \\
\hline 0.000657637 & -0.157600064 & 0.889055593 \\
\hline 0.005840253 & 0.302636723 & 0.452939088 \\
\hline 0.028038373 & 0.132361918 & 0.935027293 \\
\hline 0.042035244 & 0.118383595 & 0.788655542 \\
\hline 0.017242469 & -0.755568108 & 0.190437322 \\
\hline 0.046236001 & -0.310355268 & 0.762837691 \\
\hline 0.008859233 & 0.001066837 & 0.999188052 \\
\hline 1.18222E-06 & -1.175110165 & 0.073973802 \\
\hline 0.023335621 & 0.0555574 & 0.93849707 \\
\hline 0.031561579 & -0.079450384 & 0.957896512 \\
\hline
\end{tabular}




\begin{tabular}{|c|c|c|}
\hline 0.032697401 & 0.688208935 & 0.31276316 \\
\hline 0.00249327 & -0.070584257 & 0.952277735 \\
\hline 0.003629961 & 0.584400523 & 0.241387475 \\
\hline 0.046852148 & 0.824878455 & 0.143089223 \\
\hline 0.029709291 & 0.109393481 & 0.94179244 \\
\hline 0.00044563 & -0.461760959 & 0.387877307 \\
\hline 0.023335621 & -0.307400082 & 0.41255602 \\
\hline 0.000157282 & -0.039583476 & 0.969011697 \\
\hline 0.012598717 & 0.039609011 & 0.962044455 \\
\hline 0.028611202 & -0.445990908 & 0.481499461 \\
\hline 0.005506386 & -0.38415053 & 0.62148525 \\
\hline 0.00046548 & -0.70143702 & 0.155331251 \\
\hline 0.041100571 & 0.739990514 & 0.118739514 \\
\hline 0.000808174 & -0.299701907 & 0.665678899 \\
\hline 0.01953751 & -0.410564657 & 0.782573703 \\
\hline 0.006661276 & 0.161534799 & 0.829428306 \\
\hline 0.000638315 & -0.167801345 & 0.852564257 \\
\hline 0.00131247 & -0.070787852 & 0.917328671 \\
\hline 0.000825665 & 0.98064071 & 0.089124397 \\
\hline 0.010940804 & 0.848640758 & 0.244140776 \\
\hline 0.007946676 & 0.36984154 & 0.537272761 \\
\hline 0.029190736 & -0.001157728 & 0.999188052 \\
\hline 0.035212478 & -0.253539346 & 0.762536461 \\
\hline 0.003090924 & -0.368517533 & 0.314554428 \\
\hline 0.015496846 & -1.117810861 & 0.09766441 \\
\hline 0.034395337 & 0.036944341 & 0.956569933 \\
\hline 0.00493993 & -0.65405935 & 0.13081168 \\
\hline 0.008867603 & -0.099729189 & 0.956584257 \\
\hline 0.018497258 & -0.073480585 & 0.932310572 \\
\hline 0.007701888 & 0.012853701 & 0.994087385 \\
\hline 0.030029463 & 0.121394979 & 0.935215116 \\
\hline 1.95839E-05 & -0.793489759 & 0.158339236 \\
\hline 0.024043042 & -0.129009417 & 0.954448943 \\
\hline 0.049589673 & 0.044852796 & 0.974990923 \\
\hline 0.041376089 & 0.033404964 & 0.978829022 \\
\hline 0.005188458 & -0.212470321 & 0.853158253 \\
\hline 0.005602181 & 1.301580636 & 0.220573714 \\
\hline 0.037393167 & 0.379629983 & 0.533681232 \\
\hline 0.046467306 & 0.492570948 & 0.436290983 \\
\hline 4.08955E-06 & -0.288726801 & 0.460089169 \\
\hline 0.029174514 & -0.222622793 & 0.97631301 \\
\hline 0.000900514 & -0.028064963 & 0.973975774 \\
\hline 0.009693233 & -0.54678341 & 0.269371974 \\
\hline 0.004600566 & -0.247951441 & 0.674404764 \\
\hline 0.020703872 & -0.346943928 & 0.333829539 \\
\hline 0.000623391 & 0.600949978 & 0.547936057 \\
\hline 4.81091E-05 & -0.051556416 & 0.959425896 \\
\hline
\end{tabular}




\begin{tabular}{|c|c|c|}
\hline $8.25137 \mathrm{E}-05$ & 0.363462327 & 0.69743526 \\
\hline 0.030299209 & 0.70921475 & 0.434970443 \\
\hline 0.000477121 & 0.069076449 & 0.960088457 \\
\hline 0.003962279 & -0.171575713 & 0.757933106 \\
\hline 0.000185961 & 0.036722769 & 0.981027383 \\
\hline 0.019853563 & -0.287138827 & 0.547075277 \\
\hline 0.038841625 & 0.0838073 & 0.81118057 \\
\hline 0.005113285 & 0.252025296 & 0.826250144 \\
\hline 0.008719698 & 0.345188501 & 0.74627502 \\
\hline 0.025976041 & -0.398948993 & 0.664699942 \\
\hline 0.021299854 & 0.739066466 & 0.25958492 \\
\hline 0.000638315 & 0.33077076 & 0.487358898 \\
\hline 0.017242469 & -0.498002627 & 0.665843012 \\
\hline 0.024746688 & -0.041784754 & 0.962589633 \\
\hline 0.001311367 & -0.119588325 & 0.767171241 \\
\hline 0.001088462 & -0.814284961 & 0.44937218 \\
\hline $1.58321 \mathrm{E}-05$ & 0.508147405 & 0.626683973 \\
\hline 0.005506386 & -0.231131049 & 0.858664863 \\
\hline 0.001828957 & -0.182604214 & 0.85836927 \\
\hline 0.017904759 & -0.167700769 & 0.746997852 \\
\hline 0.000583526 & -0.269019904 & 0.718483887 \\
\hline 0.007311843 & -0.403265935 & 0.149857641 \\
\hline 0.012279148 & 1.160020381 & 0.082791767 \\
\hline 0.001739455 & -0.530236881 & 0.799742846 \\
\hline 0.03186089 & 0.16689926 & 0.754136576 \\
\hline 0.000128914 & -0.635935741 & 0.481740298 \\
\hline 0.040692213 & 0.167677269 & 0.780017796 \\
\hline 0.004292539 & -0.003097365 & 0.997529424 \\
\hline 0.044323514 & -0.51116543 & 0.744069389 \\
\hline 0.023335621 & 0.334123981 & 0.764031706 \\
\hline 0.005951129 & -0.368147628 & 0.7646352 \\
\hline 0.001365426 & 0.18092837 & 0.604727295 \\
\hline 0.001160317 & -0.142957279 & 0.943425827 \\
\hline 0.00735949 & -0.740731328 & 0.334041182 \\
\hline 0.029908822 & -0.701036553 & 0.183410388 \\
\hline 0.037393167 & -0.904430891 & 0.307538015 \\
\hline 0.012410744 & 0.218606887 & 0.768994627 \\
\hline 0.000936631 & 0.175560706 & 0.924896921 \\
\hline 0.000274281 & 0.788534892 & 0.643577188 \\
\hline 0.000900514 & -0.28442055 & 0.343595006 \\
\hline 0.045395007 & 0.172772326 & 0.899276266 \\
\hline 0.000406104 & -0.029480025 & 0.965793698 \\
\hline 0.019426867 & -0.434039082 & 0.326536514 \\
\hline 0.007663307 & 0.011258361 & 0.994091938 \\
\hline 0.0364297 & -0.804105358 & 0.057154205 \\
\hline 0.046991837 & -0.029024172 & 0.984844455 \\
\hline 0.045460992 & -0.212689296 & 0.837908527 \\
\hline
\end{tabular}




\begin{tabular}{|c|c|c|}
\hline 0.008859233 & 0.21980235 & 0.641328681 \\
\hline 0.001815965 & -0.582889627 & 0.397558407 \\
\hline 0.001443363 & -0.13612728 & 0.942752316 \\
\hline 0.000436557 & 0.020523539 & 0.965687723 \\
\hline 0.005240145 & 0.648987078 & 0.054550377 \\
\hline 0.003787885 & -0.224446068 & 0.866986051 \\
\hline 0.00462664 & -0.478613636 & 0.486471978 \\
\hline 0.0265819 & -0.059380032 & 0.965395371 \\
\hline 0.017846136 & -0.926849189 & 0.379040092 \\
\hline 0.04845752 & -0.464529931 & 0.352597412 \\
\hline 0.006593344 & -0.612657903 & 0.484158734 \\
\hline 0.013039063 & -0.004819594 & 0.997880787 \\
\hline 0.024855151 & 0.052749739 & 0.973792363 \\
\hline 0.002123539 & -0.690837258 & 0.336715167 \\
\hline 0.005754049 & 0.065292494 & 0.932165063 \\
\hline 0.045134321 & 0.005237598 & 0.993382611 \\
\hline 0.017753758 & 0.305573926 & 0.621024513 \\
\hline 0.012271924 & 0.091117171 & 0.9113261 \\
\hline 0.032432537 & 0.032273139 & 0.986777992 \\
\hline 0.008704796 & -0.489213865 & 0.0938992 \\
\hline 0.000498551 & 0.124974499 & 0.805881378 \\
\hline 0.022577663 & 0.853345617 & 0.186703471 \\
\hline 0.013939533 & 0.381575637 & 0.14586291 \\
\hline 0.031169026 & 0.171714118 & 0.860576816 \\
\hline 0.002578168 & -0.624817452 & 0.402980206 \\
\hline 0.045460992 & 0.069329707 & 0.922009705 \\
\hline 0.011884866 & -0.275782444 & 0.732100629 \\
\hline 0.00123595 & -0.529322414 & 0.456153539 \\
\hline 0.002065838 & 0.494317038 & 0.653504295 \\
\hline 0.019070637 & 0.165468001 & 0.888250591 \\
\hline 0.002218742 & -0.161633414 & 0.848697356 \\
\hline 0.024070563 & 0.074879779 & 0.97613886 \\
\hline 0.017837447 & -0.565702765 & 0.684637804 \\
\hline 0.033268917 & 0.302469121 & 0.703011767 \\
\hline 0.005790588 & -0.442627261 & 0.694814664 \\
\hline 0.003880208 & 0.292218797 & 0.649878919 \\
\hline 0.003413735 & -0.74649748 & 0.408779467 \\
\hline 0.038259174 & 0.102981184 & 0.882886237 \\
\hline 0.011625638 & 0.451269207 & 0.741812924 \\
\hline 0.024070563 & -0.215859272 & 0.804349383 \\
\hline 0.000980465 & -0.692942156 & 0.269295995 \\
\hline 0.033835141 & -0.127629905 & 0.917233217 \\
\hline 0.001546482 & 0.114442583 & 0.871047947 \\
\hline 0.000999501 & -0.683099295 & 0.202356518 \\
\hline 0.000901307 & -1.122325298 & 0.30076787 \\
\hline 0.031546005 & -0.578347539 & 0.621024513 \\
\hline 0.000700661 & -0.431376522 & 0.35777877 \\
\hline
\end{tabular}




\begin{tabular}{|c|c|c|}
\hline 0.023981552 & -0.8450685 & 0.056580423 \\
\hline 0.00069584 & -0.106675285 & 0.927611507 \\
\hline 0.009147095 & -0.206316836 & 0.835705566 \\
\hline 0.015647875 & 0.17310591 & 0.690923639 \\
\hline 0.00182502 & -0.091661669 & 0.939991451 \\
\hline 0.013702304 & 0.633017671 & 0.468289747 \\
\hline 0.032477374 & -0.630367896 & 0.24296452 \\
\hline 0.027965593 & -0.258360935 & 0.734886231 \\
\hline 0.02297612 & -0.105586197 & 0.933087338 \\
\hline 0.00502983 & -0.475957488 & 0.565707244 \\
\hline 0.027348192 & -0.090015396 & 0.927474829 \\
\hline 0.045460992 & 0.175682654 & 0.699175643 \\
\hline 0.029562874 & -0.254488236 & 0.877943331 \\
\hline 0.022807884 & -0.182920156 & 0.837032199 \\
\hline 0.000795197 & -0.828098252 & 0.171915981 \\
\hline 0.029545568 & -0.09203968 & 0.964362185 \\
\hline 0.027136656 & 0.053729555 & 0.953254268 \\
\hline 0.004137647 & -0.612109143 & 0.081082635 \\
\hline 0.036958473 & -0.410944635 & 0.843474251 \\
\hline 0.01953751 & -0.18256197 & 0.746641381 \\
\hline 0.023487716 & -0.487994372 & 0.39445159 \\
\hline 0.005980399 & -0.215275609 & 0.709046721 \\
\hline 0.022276408 & 0.280191903 & 0.328943221 \\
\hline 0.004503427 & 1.966160529 & 0.112804984 \\
\hline 0.042907697 & -0.091797784 & 0.90348464 \\
\hline 0.016323043 & 0.348195883 & 0.556938943 \\
\hline 0.00438292 & -0.149724691 & 0.805316244 \\
\hline 0.013741568 & 0.334509501 & 0.53737695 \\
\hline 0.014616597 & -0.465638588 & 0.216701127 \\
\hline 0.031632846 & -0.042794899 & 0.952185198 \\
\hline 0.04111064 & 0.9322916 & 0.12538621 \\
\hline 0.022577663 & 0.376433338 & 0.748119776 \\
\hline 0.038259174 & -0.700573535 & 0.461646396 \\
\hline 0.013829316 & -0.007444889 & 0.994595766 \\
\hline 0.019963223 & 0.193461308 & 0.748151145 \\
\hline 0.045020123 & -0.463016025 & 0.231343292 \\
\hline 0.008896115 & -0.071720797 & 0.958254307 \\
\hline 0.002139643 & -0.246673356 & 0.917846987 \\
\hline 0.045313536 & -0.202359587 & 0.724238845 \\
\hline 0.037458535 & 0.655697306 & 0.389624379 \\
\hline 0.000922085 & -0.6213534 & 0.471845336 \\
\hline 0.021502423 & -0.498189824 & 0.642314642 \\
\hline 0.043087117 & -0.174642245 & 0.806563934 \\
\hline 0.029254681 & -0.282875435 & 0.478335484 \\
\hline 0.047302553 & -0.190999745 & 0.726889473 \\
\hline 0.045840929 & 1.240114553 & 0.080677841 \\
\hline 0.023205635 & -0.067376118 & 0.944315462 \\
\hline
\end{tabular}




$\begin{array}{rrr}0.026533243 & 0.35946619 & 0.345679794 \\ 0.043648966 & -0.311143834 & 0.723711472 \\ 0.038215668 & -0.60975336 & 0.112666331 \\ 0.00638709 & 0.301275366 & 0.74330113 \\ 0.03373804 & 0.269081489 & 0.636054626 \\ 0.037622926 & 1.104599641 & 0.167841309 \\ 0.029406977 & -0.07133184 & 0.926678896 \\ 0.022126019 & 0.019095725 & 0.972308321 \\ 0.025922503 & -0.183055671 & 0.804321056 \\ 0.014394077 & -0.042228316 & 0.957896512 \\ 0.045460992 & 0.38651112 & 0.77894764 \\ 0.04557964 & -0.193157067 & 0.588569274 \\ 0.004203953 & -0.67525574 & 0.478371423 \\ 0.048646043 & 0.908993348 & 0.074897277 \\ 0.04557964 & 0.362902411 & 0.499762677 \\ 0.03366818 & -0.031770126 & 0.969956952 \\ 0.04580648 & -0.534179197 & 0.10751908 \\ 0.021502423 & -0.306600745 & 0.961860984\end{array}$




$\begin{array}{ll}\text { ProbeName } & \text { GeneSymbol } \\ \text { A_51_P277006 } & \text { Chst8 } \\ \text { A_51_P155458 } & \text { Dok7 } \\ \text { A_55_P2054350 } & \text { Fbxo44 } \\ \text { A_55_P2005055 } & \text { Pepd } \\ \text { A_66_P116461 } & \text { Mro } \\ \text { A_51_P502150 } & \text { Slc9a3r1 } \\ \text { A_55_P1967539 } & \text { Hunk } \\ \text { A_51_P209183 } & \text { Cxcl14 } \\ \text { A_55_P2127258 } & \text { Dok7 } \\ \text { A_52_P2710 } & \text { Cml5 } \\ \text { A_55_P2049211 } & \text { Pisd-ps1 } \\ \text { A_51_P475228 } & \text { Armc6 } \\ \text { A_55_P1955548 } & \text { Ezr } \\ \text { A_55_P1979330 } & \text { Dapp1 } \\ \text { A_55_P1994062 } & \text { Emx2os } \\ \text { A_55_P2054300 } & \text { Alg8 } \\ \text { A_55_P2427685 } & \text { Agl } \\ \text { A_55_P2158866 } & \text { Nme6 } \\ \text { A_55_P2181334 } & \text { 6030419C18Rik } \\ \text { A_55_P2133165 } & \text { Wwc1 } \\ \text { A_55_P2086983 } & \text { Atxn2 } \\ \text { A_55_P1965674 } & \text { Alg2 } \\ \text { A_51_P473953 } & \text { Arhgef26 } \\ \text { A_55_P2022434 } & \text { Gpi1 } \\ \text { A_55_P2030433 } & \text { Gpi1 } \\ \text { A_55_P2061809 } & \text { Ndufc2 } \\ \text { A_51_P342669 } & \text { Pgam1 } \\ & \end{array}$

\section{GeneName Description logFC AveExpr}

carbohydrate ( $\mathrm{N}$-a Mus musculus carb، 1.9987984039 .506990359

docking protein 7 docking protein $7[\leqslant 1.09970404710 .92169511$

F-box protein 44 Mus musculus F-bo: $1.094375528 \quad 11.4403973$

$\begin{array}{llll}\text { peptidase D } \quad \text { Mus musculus pept } 0.978903734 & 14.00742747\end{array}$

maestro Mus musculus mae: 0.919902196

solute carrier fami Mus musculus solut 0.874304136

hormonally upregı Mus musculus horn 0.856801394

chemokine (C-X-C Mus musculus chen 0.84718745

docking protein 7 Mus musculus dock 0.831719821

camello-like $5 \quad$ Mus musculus camı 0.791982403

phosphatidylserine Mus musculus phos 0.722973556

armadillo repeat ci Mus musculus arme 0.679278376

ezrin

Mus musculus ezrin 0.656022612

dual adaptor for pl Mus musculus dual 0.642553318

Emx2 opposite stri Mus musculus Emx: 0.603575825

asparagine-linked Mus musculus aspa 0.546261365

amylo-1,6-glucosic Mus musculus amyl 0.533416528

NME/NM23 nucler Mus musculus NME 0.497635144

RIKEN cDNA 6030 < Mus musculus RIKE 0.487781402 WW, C2 and coilec Mus musculus WW 0.43524986 ataxin 2 Mus musculus ataxi 0.427666332 asparagine-linked Mus musculus aspa $\quad 0.3917772$ Rho guanine nucle Mus musculus Rho 0.348289958 glucose phosphate Mus musculus glucc 0.336512554 glucose phosphate Mus musculus glucc 0.331171618 NADH dehydrogen Mus musculus NAD 0.267545274 phosphoglycerate Mus musculus phos 0.26250287
8.853408542

11.64877569

12.39458915

11.79136499

7.804274362

6.561499522

12.65183916

10.62667145

14.14145509

9.285773136

6.937128745

10.05023853

11.83440315

11.57278217

7.659255264

14.53699509 13.23524556

12.77511628

12.24174198

16.14117805

15.58858241

14.98538925

15.53968412 


\begin{tabular}{rrr}
\multicolumn{1}{c}{ adj.P.Val } & Orre-Log_FC & Orre-adj.P.Val \\
0.000527398 & -1.020809588 & 0.001347252 \\
0.007527662 & -1.84709202 & 0.009582176 \\
$3.56729 \mathrm{E}-05$ & -1.227709635 & 0.015712097 \\
$1.69542 \mathrm{E}-06$ & -0.729933657 & 0.028285973 \\
0.001379325 & -1.030168616 & 0.011926881 \\
0.001417212 & -1.376082523 & 0.003271452 \\
$5.70527 \mathrm{E}-07$ & -0.773139893 & 0.041430647 \\
0.008896115 & -1.724736378 & 0.001170971 \\
0.017565766 & -1.582479166 & 0.007184006 \\
$1.34579 \mathrm{E}-06$ & -1.652541234 & 0.017572167 \\
0.001443363 & -0.883124413 & 0.033965586 \\
0.001789253 & -0.676496203 & 0.045520561 \\
0.001756325 & -1.66632014 & 0.022479083 \\
0.04355309 & -0.862129878 & 0.036372988 \\
0.003464519 & -1.089412003 & 0.004521647 \\
0.001092598 & -1.123162944 & 0.003275647 \\
0.00106005 & -0.931225482 & 0.007552042 \\
0.000808174 & -0.481070518 & 0.049567233 \\
0.031413191 & -0.919446508 & 0.019012689 \\
0.037393167 & -1.066002187 & 0.038947926 \\
0.007459508 & -0.812278155 & 0.035762889 \\
0.023798032 & -0.822906418 & 0.00479841 \\
0.033356178 & -1.226972592 & 0.016222111 \\
0.022431714 & -0.53157885 & 0.025429949 \\
0.03292527 & -0.476276205 & 0.04722026 \\
0.029039158 & -0.875073385 & 0.005907455 \\
0.02976617 & -0.761186395 & 0.006498149
\end{tabular}




\begin{tabular}{|c|c|c|c|c|}
\hline ProbeName & GeneSymbol & Description & $\operatorname{logFC}$ & AveExpr \\
\hline A_52_P393314 & P2rx7 & purinergic receptor Mus musculus puriner! & -2.566483 & 9.918835431 \\
\hline A_55_P2091359 & Padi2 & peptidyl arginine $d \in$ Mus musculus peptidy & -1.825425 & 12.92514476 \\
\hline A_55_P2212603 & Apba2 & amyloid beta (A4) p Mus musculus amyloid & -1.686821 & 9.225208525 \\
\hline A_51_P355427 & Timp4 & tissue inhibitor of $r$ Mus musculus tissue ir & -1.339929 & 9.342884771 \\
\hline A_66_P125862 & A930005H10Rik & RIKEN cDNA A9300। Mus musculus RIKEN c & -1.307057 & 10.74695408 \\
\hline A_51_P337944 & Bmp2k & BMP2 inducible kin: Mus musculus BMP2 ir & -1.289259 & 10.84824895 \\
\hline A_55_P2123683 & Chrdl1 & chordin-like $1 \quad$ Mus musculus chordin & -1.06929 & 9.614315788 \\
\hline A_55_P2044242 & Slc13a5 & solute carrier famil) Mus musculus solute $\mathrm{c}$ & -0.979917 & 8.652415373 \\
\hline A_51_P474422 & Ift27 & intraflagellar transp Mus musculus intraflą & -0.888869 & 14.3953448 \\
\hline A_55_P1961014 & Selenbp1 & selenium binding pr Mus musculus seleniur & -0.846845 & 10.7388478 \\
\hline A_55_P1953387 & Fabp5 & fatty acid binding pı Mus musculus fatty aci & -0.83057 & 14.93510115 \\
\hline A_51_P331661 & Ubiad1 & UbiA prenyltransfer Mus musculus UbiA pr & -0.803178 & 11.61194832 \\
\hline A_55_P2097178 & Idua & iduronidase, alpha-l Mus musculus iduronic & -0.749596 & 9.374207676 \\
\hline A_55_P2045007 & Hrh1 & histamine receptor Mus musculus histamiı & -0.709332 & 7.351788502 \\
\hline A_51_P481159 & Cbr3 & carbonyl reductase Mus musculus carbony & -0.692289 & 12.81832935 \\
\hline A_55_P2075213 & Kbtbd11 & kelch repeat and BT Mus musculus kelch re & -0.631394 & 8.672056845 \\
\hline A_55_P2003541 & Nrcam & neuron-glia-CAM-rE Mus musculus neuron- & -0.62582 & 12.7418749 \\
\hline A_51_P312437 & Dhrs7 & dehydrogenase/red Mus musculus dehydrc & -0.621441 & 13.58264256 \\
\hline A_51_P108581 & Adrbk2 & adrenergic receptor Mus musculus adrener & -0.593173 & 9.552982166 \\
\hline A_55_P2018666 & Thrsp & thyroid hormone re Mus musculus thyroid & -0.558281 & 12.163245 \\
\hline A_55_P1961140 & Mettl3 & methyltransferase I Mus musculus methylt & -0.557766 & 11.63462664 \\
\hline A_66_P104309 & Myl2 & myosin, light polypє Mus musculus myosin, & -0.539752 & 7.415807899 \\
\hline A_55_P2063316 & Mgll & monoglyceride lipa؛ Mus musculus monogl' & -0.50398 & 13.43596113 \\
\hline A_55_P1971025 & Paqr6 & progestin and adipc Mus musculus progest & -0.501593 & 9.802427856 \\
\hline A_51_P475628 & Paqr6 & progestin and adipc Mus musculus progest & -0.470051 & 8.884532746 \\
\hline A_55_P2114863 & Mgll & monoglyceride lipa؛ Mus musculus monogl' & -0.396421 & 12.14565961 \\
\hline A_55_P1967648 & Flcn & folliculin $\quad$ Mus musculus folliculir & -0.346075 & 11.043084 \\
\hline
\end{tabular}




\begin{tabular}{rrr}
\multicolumn{1}{c}{ adj.P.Val } & Orre-Log_FC & Orre-adj.P.Val \\
\hline $1.55236 \mathrm{E}-05$ & -0.660266968 & 0.010635995 \\
$9.79076 \mathrm{E}-06$ & -1.027116115 & 0.028719125 \\
$6.98037 \mathrm{E}-05$ & -1.163609233 & 0.013387224 \\
0.032589815 & -1.42855087 & 0.001652405 \\
$4.63284 \mathrm{E}-09$ & -0.628049827 & 0.035295834 \\
$1.04732 \mathrm{E}-05$ & -0.9738674 & 0.022042658 \\
0.04580648 & -1.20652802 & 0.031366614 \\
0.005110931 & -1.80202445 & 0.000760241 \\
$6.05153 \mathrm{E}-06$ & -1.29464124 & 0.000998868 \\
0.003924362 & -1.208689375 & 0.003292044 \\
$8.32806 \mathrm{E}-05$ & -1.712356135 & 0.000684927 \\
0.00018418 & -0.856881408 & 0.021458953 \\
0.001598833 & -0.674001543 & 0.04663989 \\
$4.04131 \mathrm{E}-05$ & -1.616890585 & 0.033268661 \\
0.00382622 & -1.874528145 & 0.000448772 \\
0.031576242 & -1.32556604 & 0.022634684 \\
0.037915449 & -1.670179548 & 0.046244563 \\
0.022276408 & -0.997172138 & 0.002664326 \\
0.004939663 & -1.021467372 & 0.038072462 \\
0.010457288 & -1.643213697 & 0.000637333 \\
0.014038193 & -0.672886374 & 0.015790447 \\
0.01463281 & -0.942381393 & 0.0008304 \\
0.000509247 & -1.306765827 & 0.00174223 \\
0.021096766 & -1.10399589 & 0.012942182 \\
0.026168571 & -0.955519771 & 0.019897063 \\
0.001056578 & -1.517268335 & 0.002863738 \\
0.022807884 & -0.708070717 & 0.028413072
\end{tabular}




\begin{tabular}{|c|c|}
\hline ProbeName & GeneSymbol \\
\hline A_55_P1966838 & Xaf1 \\
\hline A_52_P516409 & Col4a6 \\
\hline A_55_P1966774 & Serpina3i \\
\hline A_66_P130813 & Samd4 \\
\hline A_52_P303176 & 1810037I17Rik \\
\hline A_55_P2132207 & 1810037I17Rik \\
\hline A_55_P2137527 & Fam183b \\
\hline A_52_P111031 & Pcdh17 \\
\hline A_55_P2026420 & Pou6f1 \\
\hline A_52_P206492 & Pop4 \\
\hline A_55_P2105944 & Olfr224 \\
\hline A_55_P1952482 & $\mathrm{Pbp} 2$ \\
\hline A_51_P462428 & Galnt15 \\
\hline A_55_P2026270 & Cfi \\
\hline A_51_P159453 & Serpina3n \\
\hline A_52_P613498 & 4833420G17Rik \\
\hline A_52_P157450 & Abhd1 \\
\hline A_51_P297105 & Ucp2 \\
\hline A_55_P2074656 & Padi2 \\
\hline A_52_P257812 & Lpl \\
\hline A_52_P597775 & Gprc5a \\
\hline A_55_P2045642 & Stmn4 \\
\hline A_66_P108247 & Ucp3 \\
\hline A_55_P2176792 & Sh3gl3 \\
\hline A_66_P105175 & Bche \\
\hline A_51_P128667 & Lynx1 \\
\hline A_51_P194249 & Stmn4 \\
\hline A_55_P1953972 & Pdhb \\
\hline A_55_P2315012 & 4930458D05Rik \\
\hline A_55_P1968276 & Tomm22 \\
\hline A_55_P2004179 & Col2a1 \\
\hline A_55_P2162344 & Lrsam1 \\
\hline A_51_P458778 & Hpgd \\
\hline A_55_P2014304 & Kank1 \\
\hline A_52_P165773 & Prmt8 \\
\hline A_55_P2093774 & Chl1 \\
\hline \multicolumn{2}{|l|}{ A_55_P2140212 } \\
\hline A_55_P2040600 & Exd1 \\
\hline A_55_P2106525 & Nmnat3 \\
\hline A_55_P2058791 & 4833420G17Rik \\
\hline A_52_P257625 & Esm1 \\
\hline A_55_P2033425 & Grm5 \\
\hline A_55_P2010936 & Fbxo17 \\
\hline A_55_P2148534 & $\mathrm{Nr} 1 \mathrm{~d} 2$ \\
\hline A_51_P129149 & Gatad2a \\
\hline
\end{tabular}

\section{GeneName Description logFC}

XIAP associated fa Mus musculus XIAP $\quad-6.156879098$ collagen, type IV, Mus musculus colla -4.328433054 serine (or cystein€ Mus musculus serir -3.517707117 sterile alpha motit Mus musculus steri -3.381084819 RIKEN cDNA 1810 Mus musculus RIKE $\quad-3.218676372$ RIKEN cDNA 1810 Mus musculus RIKE -3.150307147 family with seque Mus musculus fami -3.146347755 protocadherin 17 Mus musculus prot -3.096400623 POU domain, clas: Mus musculus POU -3.085193903 processing of prec Mus musculus proc -2.990439512 olfactory receptor Mus musculus olfac $\quad-2.853577013$ phosphatidylethaı Mus musculus phos -2.708851464 UDP-N-acetyl-alpt UDP-N-acetyl-alphe -2.327912086 complement com| Mus musculus com -2.283011075 serine (or cystein€ Mus musculus serir - 2.257787149 RIKEN cDNA 4833. Mus musculus RIKE -2.135137596 abhydrolase dom: Mus musculus abhy -2.028719361 uncoupling protei Mus musculus uncc -2.008925416 peptidyl arginine ( Mus musculus pept -1.981559202 lipoprotein lipase Mus musculus lipor -1.966798117 G protein-couplec Mus musculus G pr -1.882880186 stathmin-like 4 Mus musculus statł -1.765390964 uncoupling protei Mus musculus uncc -1.732205788 SH3-domain GRB2 Mus musculus SH3- -1.728042937 butyrylcholinester Mus musculus buty -1.719959388 Ly6/neurotoxin 1 Mus musculus Ly6/ -1.71821413 stathmin-like 4 Mus musculus statt -1.693097435 pyruvate dehydro Mus musculus pyru -1.683490724 RIKEN cDNA 4930 Mus musculus adul $\quad-1.6590401$ translocase of out Mus musculus tran: -1.623250362 collagen, type II, a Mus musculus colla -1.60138222 leucine rich repea Mus musculus leuci -1.595495677 hydroxyprostaglar Mus musculus hydr -1.568266039 KN motif and ank) Mus musculus KN n -1.562962584 protein arginine N Mus musculus prot $\quad-1.54223841$ cell adhesion molı Mus musculus cell ; - -1.534507736

0 Q3JOL9_RHOS4 (Q3 -1.53256524

exonuclease 3'-5' Mus musculus exor -1.525678034 nicotinamide nucl Mus musculus nico -1.491097654 RIKEN cDNA 4833. Mus musculus RIKE -1.485576479 endothelial cell-sk Mus musculus endc -1.476230322 glutamate receptc Mus musculus gluté -1.445020115 F-box protein 17 Mus musculus F-bo -1.431784462 nuclear receptor $\leqslant$ Mus musculus nuclı -1.418944898 GATA zinc finger c Mus musculus GAT، -1.417803996
AveExpr

9.98739195

11.49218277 8.237049671

9.712777677

12.76378133

11.50210939

10.8863997

9.286752712 8.567395379

11.37800517 8.061203017

7.72208074

10.82348441

7.59612213

11.293936

11.72763205

9.154942083

9.278059744

13.12004985

8.512458682

10.6940361

9.267843384

8.742824015

9.346406711

10.67728495

11.50331263

9.25076796

12.85410198

7.042019248

12.76321099

10.26377656

11.18370501

7.811720919 11.84753693 7.044327945 10.44255926 11.03368948 8.520248792 8.464035327 8.419571885 7.936182419 7.754319196 9.098136864 9.618498483 10.06687358 
A_51_P497100 Lgals4 lectin, galactose b Mus musculus lecti -1.394429143 A_55_P2063256 Lgals4

A_66_P125035 Duxbl2

A_51_P309854 Kcnn2

A_51_P360918 Ehd3

A_51_P497985 C2

A_52_P331762 Lmo1

A_55_P2013223 S100z

A_51_P140690 Stmn3

A_55_P2213214 A030001D20Rik

A_55_P2011659 Anapc5

A_55_P1987499 Pttg1

A_55_P1994339 Dhrs7c

A_55_P1976898 Pnp2

A_52_P650379 Strap

A_52_P447196 Col4a6

A_55_P2007673 Lrsam1

A_55_P1954724

A_55_P2029746

A_51_P241210 Lhfpl3

A_55_P1967553 Tmem254c

A_52_P441294 Chl1

A_52_P8324 Tmem178

A_55_P2027852 Ccl25

A_52_P250555 Dynll1

A_55_P2209258 B430105A11Rik

A_55_P2088720

A_55_P2110290 Tmem254a

A_51_P506937 Mrps12

A_55_P2115151 Pxylp1

A_55_P1957213 3930401B19Rik

A_55_P2133205 Nmt2

A_55_P2006035 Galnt15

A_55_P2154977 Rpap3

A_55_P2076303 March8

A_52_P1042732 Akap5

A_52_P48546 Rnf41

A_66_P122415 Snhg6

A_55_P1983958 Gm20746

A_55_P2147280 Myh1

A_52_P573255 Cdc42ep1

A_55_P2334927

A_52_P124472

A_55_P2041828

A_55_P2057622

A_55_P2079116

A_55_P2056557

lectin, galactose b Mus musculus lecti -1.384406462

doubl homeobox | Mus musculus douk -1.384107062

potassium interm Mus musculus pota -1.382825841

EH-domain contai Mus musculus EH-d -1.374346569

complement com|Mus musculus com -1.354036194

LIM domain only: Mus musculus LIM $\quad-1.330942564$

S100 calcium bind Mus musculus S10C -1.326491423

stathmin-like 3 Mus musculus statk -1.321998206

RIKEN cDNA A030 PREDICTED: Mus m -1.312346267

anaphase-promot Mus musculus anar -1.310212874

pituitary tumor-tr Mus musculus pitui -1.308267592

dehydrogenase/ $r \in$ Mus musculus deh) -1.305209065

purine-nucleoside Mus musculus purii -1.301681346

serine/threonine I Mus musculus serir -1.299661285

collagen, type IV, Mus musculus colla -1.292810345

leucine rich repea Mus musculus leuci -1.290806296
0

0

lipoma HMGIC fus Mus musculus lipor -1.284340644

transmembrane $p$ Mus musculus tran: -1.277819902

cell adhesion molı Mus musculus cell : $\quad-1.27532299$

transmembrane $p$ Mus musculus tran: -1.273076613

chemokine ( $\mathrm{C}-\mathrm{C}$ m Mus musculus cher -1.259566696

dynein light chain Mus musculus dyn $\epsilon-1.249217853$

RIKEN cDNA B430 Mus musculus 4 da' -1.246870514

0

0 Mus musculus adul -1.241041739

transmembrane $\mathrm{p}$ Mus musculus tran: -1.240039813

mitochondrial ribc Mus musculus mito -1.237578376

2-phosphoxylose |Mus musculus 2-ph -1.232574717

RIKEN cDNA 3930 Mus musculus $13 \mathrm{~d}-1.217322024$

$\mathrm{N}$-myristoyltransfi Mus musculus N-m -1.216097007

UDP-N-acetyl-alpr Mus musculus UDP $\quad-1.210849447$

RNA polymerase I Mus musculus RNA -1.208079308

membrane-associ Mus musculus men -1.204814812

A kinase (PRKA) aı Mus musculus A kir -1.204530816

ring finger protein Mus musculus ring -1.200195931

small nucleolar R^ Mus musculus smal -1.186454912

predicted gene, 21PREDICTED: Mus m -1.176907362

myosin, heavy pol Mus musculus myo -1.176209151

CDC42 effector pr Mus musculus CDC، -1.175193414

$-1.17230089$

potassium voltag€ Mus musculus pota -1.161813793

tubulin, beta 3 cla Mus musculus tubu -1.154640221

occludin/ELL dom Mus musculus 2 da' -1.118246162

0 Mus musculus cDN، -1.104652911

0 Mus musculus $12 \mathrm{~d}-1.104080784$
9.083050324

9.160897964

8.150682833

9.054592244

10.16522609

9.530156157

7.548138306

6.933102342

9.494028319

8.010692055

11.35954614

13.17460908

6.754466092

6.985190238

10.59363689

8.244840928

11.45712366

10.32515557

11.11618368

8.019613107

12.04771936

11.41555856

7.695362808

7.416547542

8.532070831

9.199220507

9.778383825

10.20028868

13.28474287

9.952113468

10.06362884

8.675213006

7.273377309

12.74712555

12.02120848

8.904980631

8.519484927

12.55778235

14.13568133

8.320743949

13.7926044

10.08795111

7.390531663

9.179596699

6.968431276

9.549861633

7.188119824 
LIM domain and a Mus musculus LIM $\quad-1.097432887$

fibulin 2

Mus musculus fibul -1.093996254

chloride channel k Mus musculus chlo| -1.093432417

BH3 interacting dc Mus musculus BH3 -1.093040424

potassium large ci Mus musculus pota -1.089176143

immunoglobulin s Mus musculus imm -1.084637957

T cell activation RI Mus musculus T cel -1.083238495

hydroxyprostaglar Mus musculus hydr -1.074317003

NLR family, CARD Mus musculus NLR $\quad-1.072914512$

RIKEN cDNA 1600 Mus musculus RIKE - -1.066481948

dynein regulatory Mus musculus dyn -1.055443982

RIKEN cDNA A730 Mus musculus RIKE - -1.054299776

olfactomedin-like Mus musculus olfac -1.047088892

zinc finger protein Mus musculus zinc -1.046286275

RIKEN cDNA 2010 Mus musculus RIKE -1.043721456

predicted gene 49 Mus musculus inhik -1.025819062

RNA polymerase I Mus musculus RNA -1.015013348

DNA cross-link rer Mus musculus DNA -1.012283165

RIKEN cDNA 6330 Mus musculus RIKE -1.011200848

RIKEN cDNA C130 Mus musculus $15 \mathrm{~d} \quad-1.010625441$

meiotic nuclear di Mus musculus meic -1.001459002

0

0 Mus musculus strai

purinergic receptc Mus musculus puriı ataxia telangiecta: Mus musculus atax solute carrier fam Mus musculus solut zinc finger protein Mus musculus zinc ring finger protein Mus musculus ring solute carrier fam Mus musculus solut minichromosome Mus musculus mini calcium channel, I Mus musculus calci apoptotic chroma Mus musculus apor malic enzyme $3, \wedge$ Mus musculus mali ATP-binding casse Mus musculus ATPa disintegrin-like a Mus musculus a dis transmembrane $p$ Mus musculus tran: calmodulin bindin Mus musculus calm malic enzyme $3, \wedge$ Mus musculus mali RIKEN cDNA 6720. Mus musculus RIKE eukaryotic transla Mus musculus euke microfibrillar-asso Mus musculus micr RIKEN cDNA 4932. Mus musculus RIKE Ly6/neurotoxin 1 Mus musculus Ly6/ lectin, galactose-b Mus musculus lecti EF-hand calcium $k$ Mus musculus EF-h protein kinase, cA Mus musculus prot predicted gene 12 Mus musculus 2 da' 1.001231056 $-0.99870721$ $-0.997462528$ $-0.992982222$ $-0.992804216$ $-0.980674207$ $-0.969683986$ $-0.968129022$ $-0.962221758$ $-0.95576469$ $-0.953796608$ $-0.95071448$ $-0.94559252$ $-0.943684784$ $-0.938806673$ $-0.938219484$ $-0.934845512$ $-0.932749376$ $-0.929622826$ $-0.927067258$ $-0.91552837$ $-0.914295845$ $-0.90932356$ $-0.908929989$ $-0.908030723$ small nuclear RNA Mus musculus smal -0.902064505
9.4342313 12.94250986 7.365545407 9.627188001 9.227035955 7.380111698 8.716056759 7.136480655 7.882077667 7.714238037 13.18253392 7.487791722 10.54004959 7.246426253 7.702890424 9.365154479 7.598731978 6.842454651 13.89378848 8.214180271 9.396695121 6.997009656 6.940510888 8.427413446 8.686096717 7.209102318 8.064939843 7.481536136 11.14341804 8.022540062 12.63445814 12.11485534 9.199235192 7.148584453 12.32166788 8.029084983 12.78076322 7.048698444 10.38326438 10.59280982 6.697441234 6.878404826 8.245668406 10.96212449 10.02342185 7.239072622 9.180275957 
A_51_P517430 Cd1d1

A_55_P1986639 3110070M22Rik

A_55_P2008722 Gm13157

A_51_P116687

A_55_P2040371

A_51_P147034

A_55_P2183914

A_55_P2072391

A_55_P1963508

A_52_P529195

A_51_P134812

A_51_P497240

A_55_P2023707

A_65_P01247

A_55_P2095909

A_52_P650325

A_52_P116264

A_66_P138584

A_55_P2059179

A_55_P2105220

A_55_P1958597

A_55_P2119892

A_55_P1998299

A_51_P408989

A_51_P348617

A_55_P2124026

A_51_P273609

A_55_P2179834

A_52_P558609

A_55_P2059986

A_51_P215374

A_55_P2105362

A_55_P2169227

A_55_P2099540

A_55_P2121156

A_55_P2187076

A_55_P2088223

A_55_P2185950

A_55_P1983959

A_55_P2185504

A_55_P1999240

A_55_P2021266

A_55_P2044602

A_55_P1955726

A_51_P254045

A_55_P2157966

A_55_P2007771
1700010I14Rik

Rnaset2a

Ica1l

Gm7120

Acp1

Slc13a5

Pcdhb4

Chac1

E330013P04Rik

Camta1

Hjurp

Lamtor3

Slc35e1

Adhfe1

Mnd1

Slc48a1

$\mathrm{Hscb}$

Slc27a3

Erbb4

Itgb4

L3hypdh

2310045N01Rik

Synj2

Itpka

Gatad2a

Clec16a

Chst14

Slc6a17

Tmed5

Ccdc184

H2afj

LOC102634215

Sncg

Synpr

Bpnt1

Ftl1

Masp2

Gm2696

Hpse

Siah3

Ptpn5

Traip

Map1a

Catsper2
CD1d1 antigen Mus musculus CD1، -0.895878116

RIKEN cDNA 3110 Mus musculus RIKE

predicted gene 13 Mus musculus pred -0.883596352

RIKEN cDNA 1700 Mus musculus RIKE

ribonuclease T2A Mus musculus ribol

islet cell autoantic Mus musculus islet

$-0.869114138$

$-0.868364731$

predicted gene 71 Mus musculus pred

acid phosphatase Mus musculus acid

$-0.864147811$

$-0.852700759$

solute carrier fam Mus musculus solut -0.850735708

protocadherin bet Mus musculus prot -0.849945399

ChaC, cation trans Mus musculus Chac -0.844774071

RIKEN cDNA E330। Mus musculus RIKE -0.844765396

calmodulin bindin Mus musculus calm - 0.835058655

Holliday junction I Mus musculus Holli

late endosomal/ly Mus musculus late

$-0.829270832$

$-0.827157354$

solute carrier fam Mus musculus solut

alcohol dehydroge Mus musculus alcol

$-0.820653163$

meiotic nuclear di Mus musculus meic

$-0.815750524$

solute carrier fam Mus musculus solut

$-0.807694136$

solute carrier fam Mus musculus solut

$-0.806533897$

v-erb-a erythroble v-erb-a erythroblas

$-0.805175492$

integrin beta 4 Mus musculus intę

L-3-hydroxyprolin Mus musculus L-3-r

$-0.797873579$

RIKEN cDNA 2310 Mus musculus RIKE

$-0.796333366$

synaptojanin 2 Mus musculus syna

$-0.791329143$

inositol 1,4,5-trisp Mus musculus inosi

$-0.788523626$

GATA zinc finger c Mus musculus GAT،

$-0.782587668$

C-type lectin dom Mus musculus C-ty!

$-0.774440114$

carbohydrate ( $\mathrm{N}-\bar{c}$ Mus musculus carb

$-0.773816524$

solute carrier fam Mus musculus solut

$-0.773417223$

transmembrane e Mus musculus tran:

$-0.771893147$

coiled-coil domair Mus musculus coile

$-0.771250879$

$\mathrm{H} 2 \mathrm{~A}$ histone famil Mus musculus $\mathrm{H} 2 \mathrm{~A}$

$-0.769429041$

uncharacterized L PREDICTED: Mus $m$

synuclein, gamma Mus musculus synu

synaptoporin Mus musculus syna

$-0.760669861$

bisphosphate 3'-n Mus musculus bispl

$-0.756583454$

ferritin light chain Mus musculus ferri

$-0.753556952$

mannan-binding li Mus musculus man

$-0.753143511$

predicted gene 26 Mus musculus pred

$-0.750089216$

heparanase

Mus musculus hepa

$-0.747821613$

seven in absentia Mus musculus seve

$-0.733318565$

$-0.726347174$

protein tyrosine $\mathrm{p}$ Mus musculus prot

TRAF-interacting $₹$ Mus musculus TRAI

$-0.721879342$

microtubule-assor Mus musculus micr

$-0.721370838$

cation channel, sp Mus musculus catic
$-0.89528171$

$-0.87477972$

$-0.82154184$

$-0.81004159$

$-0.804716433$

$-0.766437171$

$-0.76410798$

10.1105168

7.824256532

9.60656173

7.97858127

13.71026659

8.69777082

7.927760277

8.912448535

8.000988516

7.043555936

10.36841888

8.72807961

8.292413142

7.395492019

8.550491325

8.104474982

8.914064684

8.886104688

13.10075273

12.28189497

10.39221354

7.44387188

8.351358579

9.429268854

13.13596643

9.277978073

7.336973459

11.70726936

9.135758847

10.15616747

7.374150143

9.178735545

6.917791641

9.782121086

6.673007895

7.636786619

6.512934163

8.664448436

15.30605533

6.990580432

10.2949764

6.970430604

7.304939267

6.546976015

9.180452669

9.367794738

$-0.71932791$

8.371543358 
A_55_P2036813

A_55_P2106150

A_55_P1975120

A_55_P2115127

A_55_P2096368

A_51_P451588

A_55_P1979027

A_55_P1954092

A_51_P413507

A_55_P2108784

A_51_P116906

A_55_P2149951

A_51_P461108

A_55_P2169311

A_51_P477019

A_51_P394833

A_66_P106611

A_55_P1956812

A_55_P1985410

A_51_P218953

A_55_P2060991

A_51_P431870

A_55_P1984690

A_52_P434306

A_55_P2031496

A_55_P2111148

A_55_P2031692

A_52_P348031

A_51_P327874

A_52_P640922

A_55_P2045085

A_52_P1092823

A_55_P2108820

A_55_P2014100

A_55_P1953103

A_55_P2064257

A_55_P1974487

A_51_P223569

A_66_P131169

A_52_P338956

A_51_P310164

A_55_P2028936

A_55_P2181009

A_55_P2417936

A_51_P353392

A_55_P2000533

A_52_P664404
Cenpk

Gm10777

Mphosph10

LOC102632770

Plekhb1

Tmem65

2010109A12Rik

Arhgap22

Rapgef3

Prx

Osbpl10

4930515G01Rik

Rnaset2a

Tshz1

Gna14

Fam83g

Reps2

Zfp536

BC005764

Map1s

Ptprr

Rufy3

Gemin5

Gstm6

Syt9

Pth1r

Dcdc2a

Rpl34-ps1

Irx1

Adamts3

Gm7120

Nudt7

Dnaic2

Atp8b5

Ddx4

LOC100862268

Aspg

2810459M11Rik

5033425G24Rik

Gpr180

AW125324

Cript

Polk

Zfp286
0

centromere prote Mus musculus cent

$-0.714894858$

$-0.714021248$

$-0.713805022$

predicted gene 10 Mus musculus acti

$-0.71194352$

M-phase phospho Mus musculus M-p

$-0.710643542$

pleckstrin homolo Mus musculus plecl

$-0.709050858$

transmembrane $p$ Mus musculus tran:

\section{0}

0 BB713741 RIKEN fu

RIKEN cDNA 2010 Mus musculus RIKE

Rho GTPase activa Mus musculus Rho

Rap guanine nucle Mus musculus Rap

periaxin

Mus musculus peric

oxysterol binding Mus musculus oxys

RIKEN cDNA 4930 Mus musculus RIKE

ribonuclease T2A Mus musculus adul

teashirt zinc finge Mus musculus teas

guanine nucleotid Mus musculus guar

family with seque Mus musculus fami

RALBP1 associater Mus musculus RALE

zinc finger protein Mus musculus zinc

cDNA sequence Bı Mus musculus CDN

microtubule-asso( Mus musculus micr

protein tyrosine $\mathrm{p}$ Mus musculus prot

0 Q8NFW1_HUMAN

RUN and FYVE doI Mus musculus RUN gem (nuclear orga Mus musculus gem

glutathione S-tran Mus musculus glutc synaptotagmin IX Mus musculus syna parathyroid horm Mus musculus para doublecortin dom Mus musculus douk ribosomal protein Mus musculus ribos Iroquois related h Mus musculus Iroqı a disintegrin-like c Mus musculus adul predicted gene 71 Mus musculus pred nudix (nucleoside Mus musculus nudi dynein, axonemal Mus musculus dyn€ ATPase, class I, tyן Mus musculus ATP: DEAD (Asp-Glu-Ali Mus musculus DEAl uncharacterized $\mathrm{L}$ Mus musculus unct asparaginase hom Mus musculus aspa RIKEN cDNA 2810 Mus musculus RIKE RIKEN cDNA 5033. Mus musculus $11 \mathrm{~d}$ $G$ protein-couplec Mus musculus $G$ pr expressed sequen mp78f05.y1 Soares. cysteine-rich PDZ- Mus musculus cyste polymerase (DNA Mus musculus poly zinc finger protein Mus musculus zinc
$-0.701847616$

$-0.698752868$

$-0.688651928$

$-0.688137356$

$-0.685272422$

$-0.684956015$

$-0.682059235$

$-0.681579817$

$-0.680902955$

$-0.678681382$

$-0.677677589$

$-0.675928149$

$-0.674603957$

$-0.674028528$

$-0.66926502$

$-0.667960126$

$-0.667881529$

$-0.667355779$

$-0.663256388$

$-0.65688668$

$-0.655180632$

$-0.654460663$

$-0.650364411$

$-0.649492628$

$-0.648502424$

$-0.646761588$

$-0.638849777$

$-0.638107189$

$-0.637864703$

$-0.630274633$

$-0.629303175$

$-0.62905002$

$-0.628680049$

$-0.628241548$

$-0.626909774$

$-0.625547854$

$-0.624550444$

$-0.623425317$

$-0.622867594$

$-0.622037502$

$-0.622037367$

10.43484322

10.33973892

9.633629823

7.574959028

12.49347357

14.22405549

10.42426536

12.30346033

6.812251

11.21104891

13.75316692

10.13531086

7.962313068

6.703695122

7.43885386

10.80255508

8.386191975

6.64692581

8.042257813

10.56982114

7.535671867

12.34555259

6.581299389

9.649754062

10.86869213

10.40659555

9.210465339

8.327190519

8.882151868

7.587100373

14.32799093

7.349592041

6.65699864

6.806996401

10.25545194

8.390636213

6.822180076

6.622449063 
A_55_P2072656 Ckmt1

A_55_P2137941 Fxyd2

A_55_P2143251 Rims3

A_51_P253732 II17rd

A_55_P2457154 G2e3

A_51_P101573 Klc4

A_55_P2167323 Cort

A_52_P257686 Rwdd3

A_66_P111430 2410006H16Rik

A_55_P1968858 Cadps

A_52_P67270

A_55_P1970299

A_55_P2061371

A_55_P2131340

A_51_P421223

A_51_P465273

A_52_P367675

A_55_P2019113

A_55_P1968908

A_51_P151586

A_55_P2084332

A_55_P1971174

A_55_P2227580

A_55_P2223282

A_51_P230439

A_52_P211418

A_52_P357055

A_55_P1968200

A_55_P2007273

A_55_P2137701

A_55_P2000304

A_51_P192139

A_66_P128445

A_66_P122699

A_55_P1998872

A_52_P71105

A_55_P2008936

A_51_P116487

A_55_P2106235

A_66_P126415

A_52_P343627

A_51_P437478

A_55_P2053497

A_55_P2097340

A_51_P468762

A_51_P298802

A_55_P2057127
4930515G01Rik

Mttp

Gm15698

Churc1

Fv1

Mettl17

Acin1

Apol7b

Hypk

Gsg2

Pigp

$\mathrm{Cd} 1 \mathrm{~d} 2$

A130072N09Rik

B130019D13Rik

Ppfibp2

G2e3

Ccdc91

Hjurp

Pole

Gm13247

Terf1

Zfp74

Bend7

Cux2

Slitrk5

Sertad3

Slc2a9

Lsm4

Syngr2

Gm8013

Rbp7

Zfp566

Poli

Camkv

Alkbh6

Bfsp2

Nim1k creatine kinase, $m$ Mus musculus crea'

FXYD domain-con Mus musculus FXY[

$-0.61884061$

$-0.611672447$

regulating synapti regulating synaptic

$-0.607351966$

interleukin 17 reci Mus musculus inter -0.607331836

G2/M-phase speci Mus musculus G2/ $\mathrm{r}$

$-0.603883427$

kinesin light chain Mus musculus kine: -0.601913768

cortistatin

Mus musculus corti

$-0.601224344$

RWD domain cont Mus musculus RW[

$-0.599845312$

RIKEN cDNA 2410 Mus musculus RIKE

Ca2+-dependent $\subseteq$ Mus musculus Ca2-

RIKEN cDNA 4930 Mus musculus RIKE

$-0.598883168$

$-0.594774845$

microsomal triglyc Mus musculus micr

$-0.593215762$

$-0.5865943$

transcription elon Mus musculus pred

churchill domain c Mus musculus chur

$-0.584142169$

$-0.583044972$

Friend virus susce Mus musculus Frier

methyltransferas $€$ Mus musculus metl

apoptotic chroma Mus musculus apor

apolipoprotein $L 7$ Mus musculus apol

huntingtin interac Mus musculus hunt

germ cell-specific Mus musculus gern

phosphatidylinosi Mus musculus phos

CD1d2 antigen Mus musculus CD1،

RIKEN cDNA A130 Mus musculus $16 \mathrm{~d}$

RIKEN cDNA B130 Mus musculus 9.5 c

PTPRF interacting Mus musculus PTPF

G2/M-phase speci Mus musculus G2/

coiled-coil domair Mus musculus coile

Holliday junction I Mus musculus Holli

$-0.581308579$

$-0.579305966$

$-0.579116752$

$-0.572998316$

$-0.572592964$

$-0.57106147$

$-0.569703885$

$-0.567292844$

$-0.559844118$

$-0.558378263$

$-0.55630513$

$-0.554271362$

$-0.553942714$

$-0.55155621$

polymerase (DNA Mus musculus poly

$-0.550291814$

$-0.546813814$

$-0.546075343$

telomeric repeat $k$ Mus musculus telor

zinc finger protein Mus musculus ES ct

$B E N$ domain cont $B E N$ domain contai

$-0.539117791$

$-0.538424509$

cut-like homeobo: cut-like homeobox

$-0.53739685$

SLIT and NTRK-likє Mus musculus SLIT

$-0.535909484$

SERTA domain cor Mus musculus SERT

$-0.532389964$

solute carrier fam Mus musculus solut -0.532021351

LSM4 homolog, U Mus musculus LSM- -0.530560598

synaptogyrin 2 Mus musculus syna

$-0.529964886$

predicted gene 8C PREDICTED: Mus m -0.528496734

retinol binding prc Mus musculus retin -0.528055686

zinc finger protein Mus musculus zinc

$-0.525914817$

polymerase (DNA Mus musculus poly -0.523897824

CaM kinase-like vi Mus musculus CaM -0.523781288

alkB, alkylation re Mus musculus alkB,

$-0.523356552$

beaded filament $s$ Mus musculus beac

$-0.522173308$

NIM1 serine/threr Mus musculus NIM

$-0.521263457$
11.75980585

8.641529844

8.439466739

8.923142563

9.938832239

11.14096031

7.784270421

8.583303218

12.43387167

9.341019473

6.518906201

8.981610299

7.129086897

11.95122776

6.783421774

8.739388881

9.293635462

8.668444439

11.62842225

8.077335091

8.212483905

7.798990811

6.673560482

7.195029296

10.34894026

7.792367244

7.770909696

7.033226409

10.13382839

7.656052718

9.872026358

6.409237197

7.861391583

7.345768936

8.610305633

9.861783381

7.214637148

13.57404857

13.79897881

6.685350041

6.853990708

9.49558264

11.47795665

7.664345723

12.08819889

7.956404228

6.49076766 
A_55_P2145139

A_55_P2143075 Ubc

A_51_P240801 Tmem173

A_55_P2007919 Akr1c19

A_51_P331827 Slc25a41

A_55_P1975832 1810009N02Rik

A_55_P1961241 LOC102634598

A_55_P2123831 Thap6

A_55_P1968245 Ftl1

A_55_P2037812 Palmd

A_55_P1974233 Dtd2

A_55_P2045096 Hjurp

A_55_P2067513 Slc10a3

A_55_P2021892 Ccdc114

A_51_P382369 Zfp608

A_55_P2077263 Cenpk

A_55_P2018847 Crlf2

A_55_P1991500 Obfc1

A_51_P487547 Ccdc91

A_55_P2032318 4930522L14Rik

A_52_P640386 Usp53

A_66_P137383 Ddx55

A_51_P341789 Sugp1

A_51_P302942 Rasl10a

A_66_P133993 Gm5093

A_66_P128927 Gm6306

A_55_P1963687 Tsen54

A_55_P2049448 Phtf1

A_55_P2153496 Ppp2r3d

A_51_P245546 Synrg

A_55_P1967500 Nell1

A_51_P216605 Hbp1

A_55_P2157902 Igsf10

A_51_P239286 Bcl2l12

A_55_P2157360 Tagap1

A_55_P2308743 A430106A12Rik

A_55_P2115225 Fap

A_52_P327402 Cds1

A_55_P2039061 Trim12c

A_55_P2051313 Gstk1

A_52_P434841 Coa4

A_51_P291682 Tmed4

A_55_P2013203 Oxtr

A_55_P2000027 Spink2

A_52_P734742 Lrrc73

A_55_P2003951 Tmem192

A_55_P2032147 Wnt9a
0

\section{ubiquitin C}

0 predicted gene 106

transmembrane $\mathrm{p}$ Mus musculus tran:

aldo-keto reducta Mus musculus aldo

solute carrier fam Mus musculus solut

RIKEN cDNA 1810 Mus musculus RIKE

uncharacterized L PREDICTED: Mus $m$

THAP domain con Mus musculus THAI

ferritin light chain Mus musculus ferri

palmdelphin Mus musculus paln

D-tyrosyl-tRNA de Mus musculus D-ty।

Holliday junction I Mus musculus Holli

solute carrier fam Mus musculus solu1

coiled-coil domair Mus musculus 3 da'

zinc finger protein Mus musculus zinc

centromere prote Mus musculus cent

cytokine receptor. Mus musculus cyto

oligonucleotide/o Mus musculus oligc

coiled-coil domair Mus musculus coile

RIKEN cDNA 4930 Mus musculus adul

ubiquitin specific | Mus musculus ubiq

DEAD (Asp-Glu-Ali Mus musculus DEAl

SURP and $G$ patch Mus musculus SURI

RAS-like, family 1( Mus musculus RAS-

predicted gene 50 PREDICTED: Mus $m$

predicted gene 63 PREDICTED: Mus $\mathrm{m}$

tRNA splicing end، Mus musculus tRN/

putative homeodc Mus musculus puta

protein phosphat: Mus musculus prot

synergin, gamma Mus musculus syne

NEL-like 1

Mus musculus NEL-

high mobility grou Mus musculus high

immunoglobulin s Mus musculus imm

BCL2-like 12 (proli Mus musculus BCL2

$T$ cell activation $G$ Mus musculus $T$ cel

RIKEN cDNA A430 Mus musculus adul

fibroblast activatic Mus musculus fibro

CDP-diacylglycero Mus musculus CDP.

tripartite motif-co Mus musculus tripa

glutathione S-tran Mus musculus glutí

cytochrome c oxic Mus musculus cyto

transmembrane e Mus musculus tran:

oxytocin receptor Mus musculus oxyt

serine peptidase i Mus musculus serir

leucine rich repea Mus musculus leuci

transmembrane $\mathrm{p}$ Mus musculus tran:

wingless-type MN Mus musculus wing
$-0.517379933$

$-0.515250808$

$-0.513561067$

$-0.512737911$

$-0.509338122$

$-0.506346906$

$-0.503420231$

$-0.50292242$

$-0.502896156$

$-0.502660256$

$-0.498511647$

$-0.496971605$

$-0.495867287$

$-0.495838288$

$-0.493057428$

$-0.491159912$

$-0.487538832$

$-0.48748131$

$-0.486825904$

$-0.486588132$

$-0.481768787$

$-0.481484634$

$-0.480862132$

$-0.479764409$

$-0.478687639$

$-0.473064458$

$-0.472348364$

$-0.467956686$

$-0.464874953$

$-0.463458339$

$-0.461407325$

$-0.460215485$

$-0.457935852$

$-0.45634682$

$-0.456021568$

$-0.455161398$

$-0.452820717$

$-0.451307076$

$-0.448747311$

$-0.447816462$

$-0.446903622$

$-0.443998054$

$-0.442780027$

$-0.442537681$

$-0.442136942$

$-0.440106598$

$-0.439482762$
6.649375349

16.61461059

9.911571688

6.304006357

6.456710962

10.81241498

9.192490397

9.608058208

17.27428723

7.526994087

8.995692324

6.68312203

10.35448148

8.738892753

10.15897212

9.796181967

12.22311955

11.17769638

11.34420614

7.441660797

7.753841713

7.7673737

12.83928633

7.097069326

7.624410673

7.780634724

12.43909794

11.76027675

7.113971128

10.42186201

6.769094052

8.599211577

8.96305814

9.648729447

8.846243503

7.933766962

6.722753058

6.871725015

8.022101966

11.34736659

9.46071069

10.73423118

8.023385113

6.96804014 8.936830228

12.66518165

10.92706414 
A_55_P1977454

A_55_P2011692

A_52_P73559

A_52_P673499

A_55_P2025514

A_51_P122141

A_55_P2074291

A_52_P448870

A_51_P348652

A_55_P1970676

A_55_P2027152

A_55_P2330545

A_55_P1981461

A_55_P2183597

A_55_P1974477

A_55_P2153797

A_51_P372156

A_55_P2109585

A_55_P2167898

A_55_P2471798

A_52_P179729

A_52_P595642

A_51_P517001

A_52_P409457

A_55_P2069012

A_52_P108321

A_55_P2104572

A_52_P552589

A_52_P561377

A_55_P2057941

A_55_P2088711

A_51_P456838

A_51_P167374

A_55_P1967983

A_52_P47781

A_55_P2175915

A_51_P234627

A_51_P241943

A_51_P246677

A_52_P275678

A_51_P421140

A_51_P268843

A_55_P1956418

A_51_P184223

A_52_P117576

A_55_P2044967

A_51_P131025
4930570G19Rik

Gm7241

Shmt1

Pnpla3

Mamstr

Fbxo6

Rab26

Spast

Sh3yl1

Ssh1

Zfp81

5430410E06Rik

Tbc1d2

Msh3

Prnp

4930563E22Rik

Plekha7

Nat9

Snap23

Txnl4a

Smim 7

D130040H23Rik

Ppcdc

Pidd1

Ccdc71

6330416G13Rik

Map4k1

Fam160b1

1700049G17Rik

Sgsm1

Fbxl21

Gpatch1

Use1

Slitrk3

Ccl28

Nubpl

Sap30I

Rec8

Gpr135

Tubb6

Rasip1

Efr3b

Pcdhb7

Casp3

Zfp74

Ngdn
RIKEN cDNA 4930 Mus musculus RIKE 0

0 Mus musculus $12 \mathrm{~d}$

predicted pseudo! PREDICTED: Mus $\mathrm{m}$

serine hydroxyme Mus musculus serir

patatin-like phosp Mus musculus pata

MEF2 activating $n$ Mus musculus MEF

F-box protein 6 Mus musculus F-bo

RAB26, member R Mus musculus RAB:

spastin

Mus musculus spas

Sh3 domain YSC-li Mus musculus Sh3

slingshot homolo๕ Mus musculus sling

zinc finger protein zinc finger protein \&

RIKEN cDNA 5430. Mus musculus 6 da'

TBC1 domain fam Mus musculus TBC:

mutS homolog 3 (Mus musculus mut!

prion protein Mus musculus prior

RIKEN CDNA 4930 Mus musculus RIKE

pleckstrin homolo Mus musculus plecl

$\mathrm{N}$-acetyltransfera: Mus musculus $\mathrm{N}$-ac

synaptosomal-ass synaptosomal-asso

thioredoxin-like 4، Mus musculus thior

small integral mer Mus musculus smal

RIKEN cDNA D130 Mus musculus RIKE

phosphopantothe Mus musculus phos

p53 induced deatl leucine-rich and de

coiled-coil domair Mus musculus coile

RIKEN cDNA 6330. Mus musculus RIKE

mitogen-activatec Mus musculus mito

family with seque Mus musculus fami

RIKEN cDNA 1700 Mus musculus RIKE

small $G$ protein sį̨ Mus musculus smal

F-box and leucine. Mus musculus F-bo

$G$ patch domain cı Mus musculus $G$ pa

unconventional Si Mus musculus uncc

SLIT and NTRK-likє Mus musculus SLIT

chemokine ( $\mathrm{C}-\mathrm{C}$ m Mus musculus cher

nucleotide bindin̨ Mus musculus nuclı

SAP30-like

Mus musculus SAP:

REC8 meiotic reco Mus musculus REC\&

$G$ protein-couplec Mus musculus $G$ pr

tubulin, beta 6 cla Mus musculus tubu

Ras interacting prı Mus musculus Ras i

EFR3 homolog B (! Mus musculus EFR

protocadherin bet Mus musculus prot

caspase 3

Mus musculus casp

zinc finger protein PREDICTED: Mus $m$

neuroguidin, EIF4I Mus musculus neur
$-0.435903908$

$-0.434179405$

$-0.430169066$

$-0.430087668$

$-0.428814069$

$-0.427829791$

$-0.425180806$

$-0.424956589$

$-0.424725762$

$-0.424228425$

$-0.422795069$

$-0.422072903$

$-0.420509947$

$-0.420158886$

$-0.420019862$

$-0.416155848$

$-0.411011206$

$-0.40841751$

$-0.407279096$

$-0.407062662$

$-0.406120836$

$-0.406112892$

$-0.400511742$

$-0.398509715$

$-0.397772469$

$-0.396032959$

$-0.394704756$

$-0.3923582$

$-0.38798432$

$-0.383331981$

$-0.3821107$

$-0.379835667$

$-0.37911143$

$-0.3789746$

$-0.376113158$

$-0.374930748$

$-0.373125451$

$-0.373063734$

$-0.367071601$

$-0.36573505$

$-0.364131056$

$-0.361460853$

$-0.360229036$

$-0.356613104$

$-0.351742878$

$-0.350202078$

$-0.340262798$
7.161056162

8.677359589

9.586233165

7.142456297

6.700148086

6.52676216

13.84031149

8.087186472

12.20201077

9.598710633

6.834679035

8.529420477

6.602185969

10.38577948

10.7021489

10.31468171

7.679272315

10.278925

7.689265574

10.13514035

12.55293121

10.50763342

6.338211044

6.177407482

9.97175954

8.637270977

8.641996503

8.471880485

12.37367207

7.964018539

6.60586313

6.874468646

9.9860935

13.46950848

6.396932242

6.151763833

10.36569262

14.28047258

8.342925093

6.810300528

14.78805775

6.858814738

9.792465029

7.918822651

8.091329134

6.389182029

13.42970696 


$\begin{array}{ll}\text { A_55_P2067131 } & \text { Dclre1c } \\ \text { A_55_P2027077 } & \text { Shc2 } \\ \text { A_55_P2026054 } & \text { Usp37 } \\ \text { A_55_P2012096 } & \text { Bmp8a } \\ \text { A_55_P2143499 } & \text { Pgbd5 } \\ \text { A_55_P1999829 } & \text { Thoc7 } \\ \text { A_51_P244558 } & \text { Rab3gap2 } \\ \text { A_55_P2002113 } & \text { Rpl36 } \\ \text { A_51_P289414 } & \text { Spg11 } \\ \text { A_52_P133578 } & \text { Gpr158 } \\ \text { A_66_P130612 } & \text { 2810408A11Rik } \\ \text { A_51_P208377 } & \text { Trappc5 } \\ \text { A_55_P2177233 } & \text { Abhd5 } \\ \text { A_55_P1968683 } & \text { Anks1b } \\ \text { A_51_P288505 } & \text { Tradd } \\ \text { A_51_P133747 } & \text { Ppp1r3e } \\ \text { A_51_P476711 } & \text { Skiv2l2 } \\ \text { A_55_P2041372 } & \text { Gm3693 } \\ \text { A_51_P242356 } & \text { Fam114a2 } \\ \text { A_51_P146303 } & \text { Mvb12a } \\ \text { A_55_P1960621 } & \text { Gm20764 } \\ \text { A_51_P107433 } & \text { Mrpl34 }\end{array}$

DNA cross-link rer Mus musculus DNA $\quad-0.338402188 \quad 6.315412162$

SHC (Src homolog Mus musculus SHC $\quad-0.33820507 \quad 7.375840756$ ubiquitin specific|Mus musculus ubiq $-0.333676116 \quad 6.919608859$ bone morphogenє Mus musculus bont $-0.330118616 \quad 7.33400305$ piggyBac transpos Mus musculus pigg' $\quad-0.32667779 \quad 6.992213644$ THO complex 7 hc Mus musculus THO $-0.324738732 \quad 13.25310654$ RAB3 GTPase actii Mus musculus RAB: $\quad-0.320028198 \quad 11.01221532$ ribosomal protein Mus musculus ribos -0.30828050616 .65947439$ spastic paraplegia Mus musculus spas $\quad-0.306241068 \quad 11.47302291$ G protein-couplec Mus musculus G pr $\quad-0.302885456 \quad 6.416264013$ RIKEN cDNA 2810. Mus musculus RIKE $\quad-0.30270723 \quad 7.686468818$ trafficking protein Mus musculus traff $-0.297651608 \quad 13.16186953$ abhydrolase domi Mus musculus abhy $\quad-0.2762267199 .274185661$ ankyrin repeat anı Mus musculus anky -0.2758419656 .319708996$ TNFRSF1A-associa Mus musculus TNFF $\quad-0.27415141 \quad 11.49981129$ protein phosphat: Mus musculus prot $-0.272996573 \quad 6.29524283$ superkiller viraliciı Mus musculus supe $-0.270984564 \quad 11.41603014$ predicted gene 36 PREDICTED: Mus m $\quad-0.2646902 \quad 6.199455621$ family with seque Mus musculus fami $\quad-0.262671874 \quad 12.80714837$ multivesicular boc Mus musculus mult $\quad-0.260071046 \quad 12.17524294$ predicted gene, 21 PREDICTED: Mus m -0.258454404 15.49930838 mitochondrial ribc Mus musculus mito $-0.248200928 \quad 12.30902572$ 


\begin{tabular}{|c|c|c|}
\hline adj.P.Val & Orre-Log_FC & Orre-adj.P.Val \\
\hline $1.65288 \mathrm{E}-09$ & 1.142724869 & 0.160126428 \\
\hline $1.68799 \mathrm{E}-16$ & 1.678279674 & 0.148286687 \\
\hline 0.00111415 & 0.276521938 & 0.856285005 \\
\hline $3.4835 \mathrm{E}-12$ & 0.849713018 & 0.247609338 \\
\hline $9.75281 \mathrm{E}-16$ & 0.065397702 & 0.920220832 \\
\hline $8.73791 E-16$ & 0.032101359 & 0.971052049 \\
\hline 4.37739E-06 & 1.601979349 & 0.136939403 \\
\hline 4.62079E-09 & -1.246342593 & 0.095148351 \\
\hline $1.53107 \mathrm{E}-11$ & -0.4348927 & 0.346208983 \\
\hline 1.19839E-16 & -0.29463285 & 0.553202296 \\
\hline $2.76734 \mathrm{E}-14$ & -0.227918918 & 0.76436986 \\
\hline $5.32555 \mathrm{E}-10$ & -0.565335125 & 0.64712269 \\
\hline 1.10279E-05 & 0.963523397 & 0.221847465 \\
\hline 0.00086345 & 0.372087289 & 0.620916602 \\
\hline 0.022418234 & 1.275249124 & 0.081367361 \\
\hline $2.19306 \mathrm{E}-10$ & -0.399166545 & 0.510246289 \\
\hline $1.9464 \mathrm{E}-13$ & -0.671109095 & 0.1423435 \\
\hline 0.003448159 & 0.710352137 & 0.080953995 \\
\hline $2.85216 \mathrm{E}-06$ & -1.037247785 & 0.050508329 \\
\hline 0.000396451 & 1.606931711 & 0.058847733 \\
\hline 4.87063E-05 & 0.057324928 & 0.930977484 \\
\hline 0.016968618 & 0.665876263 & 0.227829936 \\
\hline 0.005579854 & 0.288125691 & 0.591016158 \\
\hline $1.20027 \mathrm{E}-10$ & 0.852487476 & 0.203223242 \\
\hline $1.00373 \mathrm{E}-08$ & 0.87696271 & 0.228263976 \\
\hline $8.90582 \mathrm{E}-07$ & 0.177948445 & 0.737984738 \\
\hline 0.032298961 & 0.680503994 & 0.232991983 \\
\hline $1.47938 \mathrm{E}-09$ & -0.612991048 & 0.078478102 \\
\hline 1.12499E-07 & 0.619121571 & 0.164250851 \\
\hline $5.03003 \mathrm{E}-15$ & 0.191531928 & 0.600619589 \\
\hline 0.030428182 & -0.154790328 & 0.854360891 \\
\hline 0.000514259 & -0.421653429 & 0.122025146 \\
\hline 0.004381404 & 0.725679062 & 0.37780821 \\
\hline 8.03792E-08 & 0.147025135 & 0.90705714 \\
\hline 0.003583798 & 0.9132766 & 0.171140015 \\
\hline 0.001739455 & -0.273496341 & 0.863827852 \\
\hline $6.69066 \mathrm{E}-07$ & -0.091756023 & 0.893142157 \\
\hline $3.00086 \mathrm{E}-06$ & -1.012174185 & 0.25474915 \\
\hline 4.59997E-05 & -0.757915491 & 0.066325331 \\
\hline $1.17782 \mathrm{E}-08$ & -0.353650207 & 0.70042947 \\
\hline 0.001772617 & -0.112859725 & 0.962512328 \\
\hline 0.029882996 & 0.594754598 & 0.427097363 \\
\hline 8.79673E-09 & -0.265966628 & 0.529308318 \\
\hline 1.10279E-05 & -0.675059016 & 0.223691543 \\
\hline 1.11591E-09 & 0.421854979 & 0.289475858 \\
\hline
\end{tabular}




\begin{tabular}{|c|c|c|}
\hline 0.009286241 & -0.295782195 & 0.686189715 \\
\hline 0.011447725 & -0.305899627 & 0.691832878 \\
\hline 2.57183E-07 & -0.613424673 & 0.283946905 \\
\hline 0.000124631 & -1.584282679 & 0.053011793 \\
\hline 1.02537E-05 & 0.628280133 & 0.408457847 \\
\hline 0.030972985 & -0.559354504 & 0.187239299 \\
\hline 0.02720596 & -1.230463843 & 0.186703471 \\
\hline 0.004452627 & 0.051163701 & 0.969437555 \\
\hline 0.026361683 & 0.545463528 & 0.417027811 \\
\hline $3.1151 \mathrm{E}-07$ & -0.364747065 & 0.56625963 \\
\hline 4.60298E-08 & 0.662787674 & 0.052881943 \\
\hline 8.90883E-09 & -0.14863164 & 0.756692535 \\
\hline 0.000380425 & 0.088500281 & 0.969964566 \\
\hline $2.95281 \mathrm{E}-10$ & -0.526342386 & 0.511302615 \\
\hline $2.0813 \mathrm{E}-07$ & 0.044819377 & 0.953255298 \\
\hline 1.23069E-07 & -0.071202692 & 0.882404727 \\
\hline 0.004785393 & -0.403521443 & 0.091993005 \\
\hline 1.29869E-07 & 0.04377789 & 0.9628957 \\
\hline 1.87675E-06 & -0.11163067 & 0.888541003 \\
\hline 0.038078984 & -0.360765364 & 0.681260954 \\
\hline 9.48239E-05 & -0.502842706 & 0.160623939 \\
\hline 0.006661276 & -0.528933679 & 0.707697644 \\
\hline $1.24605 \mathrm{E}-05$ & 0.616650301 & 0.144912553 \\
\hline 0.000338177 & -0.570124245 & 0.474083165 \\
\hline 8.64891E-08 & 0.27076903 & 0.802552215 \\
\hline $6.28876 \mathrm{E}-05$ & -0.539482757 & 0.457925459 \\
\hline 0.000177844 & -0.083079696 & 0.925518302 \\
\hline $6.73139 \mathrm{E}-05$ & -0.346003991 & 0.778235249 \\
\hline 8.02961E-12 & -0.265245317 & 0.354709911 \\
\hline 1.13328E-08 & 0.295428837 & 0.90816598 \\
\hline 1.12499E-07 & 0.03003259 & 0.973326255 \\
\hline $2.22782 \mathrm{E}-06$ & -0.093002614 & 0.949245873 \\
\hline 0.000213749 & 0.909025335 & 0.386634945 \\
\hline 8.8279E-07 & -0.026648062 & 0.964173244 \\
\hline $2.82752 \mathrm{E}-06$ & 0.416415697 & 0.24076864 \\
\hline $3.56596 \mathrm{E}-07$ & 0.113710815 & 0.932303384 \\
\hline 0.000235777 & 0.058359829 & 0.960840137 \\
\hline 2.89973E-05 & 0.21152987 & 0.456262298 \\
\hline 1.18222E-06 & 0.347243353 & 0.287095476 \\
\hline 0.028177835 & 0.107025665 & 0.960938727 \\
\hline 2.33153E-08 & -0.14847078 & 0.866071086 \\
\hline 8.83882E-05 & -0.725308474 & 0.385110835 \\
\hline 0.001479941 & -0.957921963 & 0.121507432 \\
\hline 0.020221663 & -1.000079548 & 0.088013122 \\
\hline 0.000989719 & 0.181901546 & 0.865463843 \\
\hline 0.044450672 & -0.832723385 & 0.174878739 \\
\hline $5.25828 \mathrm{E}-07$ & -0.878381793 & 0.330185942 \\
\hline
\end{tabular}




\begin{tabular}{|c|c|c|}
\hline 0.002522403 & 0.849196584 & 0.11410773 \\
\hline 0.034978678 & 1.066752424 & 0.061811889 \\
\hline 5.16704E-06 & 0.228183729 & 0.803256511 \\
\hline 0.00045366 & 0.269147757 & 0.620916602 \\
\hline 0.007902317 & 0.826185911 & 0.218557489 \\
\hline 0.029919663 & -0.801326888 & 0.221233965 \\
\hline 8.1122E-08 & 0.327987134 & 0.529096273 \\
\hline 0.021399671 & 0.212515961 & 0.816645104 \\
\hline 0.023855751 & 1.042514284 & 0.073196754 \\
\hline $9.10813 \mathrm{E}-08$ & 0.462573009 & 0.435684592 \\
\hline 0.000879567 & 0.556041176 & 0.24217521 \\
\hline 8.45947E-07 & -0.36270771 & 0.67416452 \\
\hline 0.000940356 & -1.11932272 & 0.077538087 \\
\hline 7.78121E-07 & -0.49529212 & 0.639877775 \\
\hline 1.86279E-07 & -0.535744497 & 0.763800599 \\
\hline $2.66078 \mathrm{E}-06$ & -0.599168408 & 0.455341412 \\
\hline 1.11113E-06 & -0.431074839 & 0.713563846 \\
\hline 1.146E-09 & -0.412211363 & 0.623298435 \\
\hline 0.000514259 & 0.007129223 & 0.994595766 \\
\hline 4.25257E-06 & -0.906668501 & 0.187157373 \\
\hline 4.0172E-06 & 0.868287968 & 0.462835421 \\
\hline $6.43246 \mathrm{E}-09$ & -0.045700265 & 0.957349204 \\
\hline 0.000808877 & -0.463734507 & 0.483573822 \\
\hline 5.91303E-07 & -0.107053089 & 0.955717495 \\
\hline 0.012006226 & 0.956031506 & 0.075117433 \\
\hline 8.8279E-07 & -0.146960514 & 0.937741007 \\
\hline 4.04338E-05 & 0.494367531 & 0.707273068 \\
\hline 0.001494303 & -0.86883404 & 0.111021145 \\
\hline 2.49994E-05 & 0.315838681 & 0.299230857 \\
\hline 0.021283873 & 0.701831504 & 0.486599911 \\
\hline 0.000787448 & -0.211887665 & 0.701618101 \\
\hline 0.004873422 & -0.16006652 & 0.822085473 \\
\hline 0.00065398 & 0.705026697 & 0.149580938 \\
\hline 0.006646131 & 0.07136628 & 0.93329 \\
\hline $1.26079 \mathrm{E}-06$ & 0.62528796 & 0.207764268 \\
\hline 0.00422092 & 0.687410102 & 0.348274964 \\
\hline 0.017320417 & -0.123620704 & 0.93274864 \\
\hline 0.000865869 & -0.545100208 & 0.61634742 \\
\hline 0.004072369 & 0.634433589 & 0.522202668 \\
\hline 0.000139969 & 0.190076477 & 0.782540795 \\
\hline 0.000920963 & 0.427011528 & 0.662385586 \\
\hline $2.07012 \mathrm{E}-07$ & 0.106961267 & 0.910425311 \\
\hline $1.25841 \mathrm{E}-05$ & 0.003710877 & 0.997529424 \\
\hline 0.000160711 & -0.481064873 & 0.612373412 \\
\hline 0.024168112 & 1.14716478 & 0.181086511 \\
\hline $2.68225 \mathrm{E}-07$ & -0.294849957 & 0.42823224 \\
\hline 8.09056E-05 & 0.084060238 & 0.936683764 \\
\hline
\end{tabular}




\begin{tabular}{|c|c|c|}
\hline 0.007663307 & -0.799612857 & 0.087898753 \\
\hline 5.17071E-06 & 0.028982082 & 0.974657389 \\
\hline $1.40072 \mathrm{E}-05$ & 0.671385905 & 0.358784597 \\
\hline 7.34401E-09 & -0.185177946 & 0.994687287 \\
\hline $1.70362 \mathrm{E}-05$ & -0.153604513 & 0.658218205 \\
\hline 0.020608568 & 0.523662472 & 0.164475504 \\
\hline $5.3738 \mathrm{E}-05$ & -0.269786955 & 0.886173565 \\
\hline $1.27463 \mathrm{E}-06$ & 0.234373467 & 0.785625151 \\
\hline 0.003962279 & -1.658537825 & 0.174152657 \\
\hline $2.1228 \mathrm{E}-05$ & -0.785319459 & 0.333027028 \\
\hline 0.002858407 & 0.696637811 & 0.215594182 \\
\hline 0.029201966 & -1.029696787 & 0.181302122 \\
\hline 0.049928301 & 0.728811047 & 0.434228048 \\
\hline $9.70552 \mathrm{E}-06$ & -0.267104034 & 0.803228826 \\
\hline 0.00072474 & 0.119862456 & 0.952728519 \\
\hline 0.000194832 & 0.292638801 & 0.661185095 \\
\hline 0.00106562 & -0.939166723 & 0.221631516 \\
\hline 0.000343269 & 0.741731038 & 0.522632048 \\
\hline 0.004781667 & -0.366905188 & 0.117899055 \\
\hline 0.003049657 & -0.341114529 & 0.126943494 \\
\hline 0.019104179 & 0.652007915 & 0.43452554 \\
\hline 0.002510213 & -0.751272165 & 0.336696332 \\
\hline 0.002006738 & 0.814092784 & 0.264353543 \\
\hline $3.09102 \mathrm{E}-06$ & -0.363507293 & 0.625172597 \\
\hline 0.000226702 & 0.156997633 & 0.726845359 \\
\hline 0.001739455 & 0.834649216 & 0.063338074 \\
\hline $5.89518 \mathrm{E}-05$ & -0.163584726 & 0.830946743 \\
\hline 4.13642E-05 & 0.675810424 & 0.052881943 \\
\hline $1.36808 \mathrm{E}-05$ & -0.298528624 & 0.766520964 \\
\hline 0.000946017 & 0.176759778 & 0.80965285 \\
\hline 0.000513175 & 1.550754844 & 0.12547417 \\
\hline 0.00765806 & -0.766589405 & 0.52212154 \\
\hline 0.000477922 & -0.20109441 & 0.859532605 \\
\hline 0.000274281 & 0.202085117 & 0.716982172 \\
\hline 0.003787885 & -0.320302848 & 0.796581787 \\
\hline 0.000521962 & 1.360014488 & 0.10709461 \\
\hline 0.004880303 & 0.862857734 & 0.373386265 \\
\hline 0.000348616 & -0.416912256 & 0.721126308 \\
\hline 0.001552902 & 0.297216072 & 0.218608045 \\
\hline 0.001177609 & -0.623807483 & 0.375028914 \\
\hline 0.00890242 & -0.094315912 & 0.844975737 \\
\hline 0.00772897 & 1.411452151 & 0.073164284 \\
\hline $2.21618 \mathrm{E}-05$ & -0.0299814 & 0.962982937 \\
\hline 0.042698802 & -0.845915365 & 0.362927592 \\
\hline 0.006862966 & -0.592263973 & 0.335614812 \\
\hline 0.000286965 & 0.135677751 & 0.845628431 \\
\hline 0.04360833 & -0.634643815 & 0.281681955 \\
\hline
\end{tabular}




\begin{tabular}{|c|c|c|}
\hline 0.002352939 & 0.537367839 & 0.319240532 \\
\hline 0.001838118 & 0.099328743 & 0.965738256 \\
\hline 2.61434E-05 & -0.580371835 & 0.128047507 \\
\hline 0.00166382 & 0.169980767 & 0.924413675 \\
\hline 0.001053055 & 0.596935662 & 0.291364104 \\
\hline 0.003868229 & -0.790273395 & 0.061903151 \\
\hline 0.003464519 & 0.06891614 & 0.940965161 \\
\hline 0.014137811 & 0.678359418 & 0.149406144 \\
\hline 0.00048132 & -0.005507094 & 0.995037772 \\
\hline 0.005884175 & -0.000641415 & 0.999188052 \\
\hline 0.033061509 & -1.173235205 & 0.072464531 \\
\hline 0.028183984 & 1.273370764 & 0.053918806 \\
\hline 0.003878745 & 0.773465828 & 0.136838677 \\
\hline 3.67631E-06 & -0.250196249 & 0.730176352 \\
\hline $4.67766 \mathrm{E}-05$ & -0.193793319 & 0.823862712 \\
\hline 0.010314396 & 0.325885265 & 0.685160061 \\
\hline 0.002240467 & 1.152263142 & 0.146354644 \\
\hline 0.000285574 & 0.378889794 & 0.699928626 \\
\hline $9.19636 \mathrm{E}-05$ & 0.510347831 & 0.523508617 \\
\hline 0.042062516 & 0.080698546 & 0.943489946 \\
\hline 0.029423564 & 0.934807474 & 0.191076752 \\
\hline 0.000787448 & -0.255616985 & 0.366479729 \\
\hline 0.000481993 & 1.355335776 & 0.10751908 \\
\hline 0.026508362 & -0.007114081 & 0.995331835 \\
\hline 0.009390759 & -0.758024335 & 0.315758931 \\
\hline 0.00191871 & -0.146261428 & 0.677327414 \\
\hline 0.001769598 & -0.679286967 & 0.130688021 \\
\hline 0.002858407 & -1.358564611 & 0.470354265 \\
\hline 0.017812734 & 0.99453454 & 0.288434003 \\
\hline 0.034713642 & 0.086919777 & 0.920220832 \\
\hline 0.002850385 & 0.66253016 & 0.674404764 \\
\hline 0.045840929 & -0.402373451 & 0.781449844 \\
\hline 0.033043056 & -1.234861806 & 0.501844606 \\
\hline 0.000260743 & -0.280319878 & 0.575827905 \\
\hline 0.045599099 & -0.851254047 & 0.063254458 \\
\hline 0.002541053 & -0.248993552 & 0.816984648 \\
\hline 0.033835141 & -0.336948904 & 0.788558621 \\
\hline 0.00011653 & -0.418256406 & 0.392745892 \\
\hline 0.012006226 & -1.278191806 & 0.118739514 \\
\hline 0.041100571 & 1.151585772 & 0.052723907 \\
\hline 0.002510213 & -0.848286438 & 0.257895081 \\
\hline 0.002730458 & -0.346497835 & 0.60105635 \\
\hline 0.00011653 & -0.213543303 & 0.847281021 \\
\hline 0.023007997 & 0.155785852 & 0.846495297 \\
\hline 0.000674134 & -0.041710334 & 0.95306947 \\
\hline 0.002881586 & 0.619262352 & 0.543422253 \\
\hline 0.000140998 & -0.901765436 & 0.145010359 \\
\hline
\end{tabular}




\begin{tabular}{|c|c|c|}
\hline 0.002176214 & -0.095271676 & 0.871431046 \\
\hline 0.001991538 & 0.48159877 & 0.258961956 \\
\hline 0.027324146 & -0.369490654 & 0.624907028 \\
\hline 0.007527662 & 0.342720668 & 0.663728782 \\
\hline 0.020217114 & -0.270696356 & 0.730176271 \\
\hline 4.5911E-06 & 0.090277607 & 0.850567703 \\
\hline 0.000661633 & -0.375116071 & 0.376961844 \\
\hline 0.028553269 & -0.494162913 & 0.574010703 \\
\hline 0.008685806 & 0.375827546 & 0.197666255 \\
\hline 0.013601838 & 0.122358191 & 0.898443164 \\
\hline 0.000323863 & -0.116362408 & 0.860738453 \\
\hline 0.001301568 & -0.312938927 & 0.787463434 \\
\hline 8.22893E-05 & 0.170140193 & 0.921782071 \\
\hline 0.003210504 & 0.259876732 & 0.438452738 \\
\hline 0.000514259 & -0.277241027 & 0.621873235 \\
\hline 0.000260743 & -0.076770896 & 0.950362378 \\
\hline 0.007172418 & -0.075961804 & 0.917867404 \\
\hline 0.006429046 & 0.031392711 & 0.980311264 \\
\hline 0.015764004 & 0.363949983 & 0.320973793 \\
\hline 0.012348642 & 0.14592951 & 0.876257923 \\
\hline 0.037707131 & -0.650754337 & 0.494334927 \\
\hline 0.007993662 & -0.292873763 & 0.747474484 \\
\hline 0.006859216 & -0.219000675 & 0.89778475 \\
\hline 0.000797928 & -0.242679997 & 0.757718301 \\
\hline 0.021112606 & -0.14289943 & 0.848697356 \\
\hline 0.021637715 & -0.154867287 & 0.944533634 \\
\hline 0.019045055 & 0.602354516 & 0.427097363 \\
\hline 0.015607478 & -0.140469787 & 0.905219448 \\
\hline 0.013025023 & -0.354096889 & 0.723642875 \\
\hline 0.003090152 & -0.139223339 & 0.877278291 \\
\hline 0.001913361 & 0.213873744 & 0.724262095 \\
\hline 0.004785393 & -0.219696937 & 0.841493976 \\
\hline 0.04119468 & 0.1570334 & 0.848697356 \\
\hline 0.000425856 & -0.284125666 & 0.605869922 \\
\hline 0.01653793 & -1.194971489 & 0.084520342 \\
\hline 0.022158916 & -0.240595816 & 0.742005943 \\
\hline 0.000542704 & 0.467797447 & 0.570206141 \\
\hline 0.005407874 & 0.393055825 & 0.12055488 \\
\hline 0.000482925 & 0.532179195 & 0.080234718 \\
\hline 0.000115359 & 0.801928198 & 0.380687411 \\
\hline 0.027688077 & -0.478976559 & 0.718152474 \\
\hline 0.00626386 & -0.517260931 & 0.123316917 \\
\hline 0.019650551 & -0.286561287 & 0.491010741 \\
\hline 0.04557964 & 0.377740907 & 0.746641381 \\
\hline 0.030153144 & 0.13577772 & 0.686886526 \\
\hline 0.041662717 & -0.276054081 & 0.63577198 \\
\hline 0.004162645 & -1.005040354 & 0.166109453 \\
\hline
\end{tabular}




\begin{tabular}{|c|c|c|}
\hline 0.017101652 & -0.020456264 & 0.980974266 \\
\hline 0.001826457 & -0.255143155 & 0.595695059 \\
\hline 0.020615605 & -0.068581325 & 0.903742757 \\
\hline 0.014657171 & -0.373089331 & 0.572426694 \\
\hline 7.18774E-05 & -0.749372736 & 0.270791925 \\
\hline 0.017983828 & -0.335332834 & 0.371033405 \\
\hline 0.011985314 & -1.050870318 & 0.168238154 \\
\hline 0.005449864 & -0.412169954 & 0.687700428 \\
\hline 0.001724246 & -0.017363805 & 0.972308321 \\
\hline 0.043349698 & 0.661116342 & 0.50677422 \\
\hline 0.009341844 & 0.014145369 & 0.98851048 \\
\hline 0.017101652 & -0.312552559 & 0.700453503 \\
\hline 0.003987601 & 0.417026779 & 0.162591324 \\
\hline 0.003787885 & -0.272331501 & 0.735415385 \\
\hline 0.010600787 & -0.573014145 & 0.159828032 \\
\hline 0.00945011 & 0.410455624 & 0.648321778 \\
\hline 0.023855751 & 0.439018315 & 0.105716718 \\
\hline 0.023806401 & 0.330493939 & 0.324324776 \\
\hline 0.023431976 & 0.683448852 & 0.207046344 \\
\hline 0.00616295 & -0.155556489 & 0.913752916 \\
\hline 0.006924917 & 0.29181634 & 0.759460171 \\
\hline 0.048012898 & -0.453884017 & 0.563235386 \\
\hline 0.001299081 & 0.023107602 & 0.95527923 \\
\hline 0.010301701 & 0.240262954 & 0.800273179 \\
\hline 0.007124108 & 0.087532184 & 0.930824187 \\
\hline 0.004312655 & -0.103611753 & 0.893460837 \\
\hline 0.018497258 & -0.068005788 & 0.88829473 \\
\hline 0.015496846 & 0.152089146 & 0.869910406 \\
\hline 0.003849801 & 0.346927894 & 0.795848217 \\
\hline 0.024841441 & 0.217045023 & 0.471401257 \\
\hline 0.013999126 & 0.626241783 & 0.379023915 \\
\hline 0.049458458 & -0.646266334 & 0.247609338 \\
\hline 0.016394025 & 0.46227378 & 0.611574175 \\
\hline 0.038353074 & -0.229113621 & 0.62171436 \\
\hline 0.004382502 & 0.471411262 & 0.295336754 \\
\hline 0.047627976 & -0.436165386 & 0.472872632 \\
\hline 0.020805431 & 1.08588179 & 0.258168507 \\
\hline 0.033356178 & 0.95926223 & 0.181086511 \\
\hline 0.03327132 & 0.531505036 & 0.497571846 \\
\hline 0.001997079 & -0.452585095 & 0.26155099 \\
\hline 0.014533428 & -0.301472041 & 0.414746594 \\
\hline 0.000645478 & -0.105064776 & 0.889718554 \\
\hline 0.031479346 & -0.504360067 & 0.575013211 \\
\hline 0.033404783 & -0.94966159 & 0.479502812 \\
\hline 0.026533243 & -0.438472552 & 0.446475861 \\
\hline 0.03239946 & -0.0774077 & 0.846672512 \\
\hline 0.03366818 & -0.255451735 & 0.817224457 \\
\hline
\end{tabular}




\begin{tabular}{|c|c|c|}
\hline 0.003868229 & -0.705241745 & 0.135046744 \\
\hline 0.010074878 & -0.498865268 & 0.64759635 \\
\hline 0.007060905 & -0.116108895 & 0.922009705 \\
\hline 0.001081614 & -0.058486427 & 0.965738256 \\
\hline 0.046707673 & -0.164545305 & 0.817302191 \\
\hline 0.011549849 & 0.583828781 & 0.133878139 \\
\hline 0.02006507 & 0.166117733 & 0.581252142 \\
\hline 0.04564252 & 0.278102326 & 0.792689996 \\
\hline 0.021600942 & -0.061664499 & 0.937846213 \\
\hline 0.035885563 & -0.12149351 & 0.906045122 \\
\hline 0.024269528 & 0.200648873 & 0.851391995 \\
\hline 0.019991441 & 0.056133517 & 0.969713104 \\
\hline 0.038760156 & 0.266715159 & 0.66770638 \\
\hline 0.024043042 & -0.54078503 & 0.683080867 \\
\hline 0.024111685 & 0.362224632 & 0.597324086 \\
\hline 0.015600748 & -0.444145898 & 0.467954352 \\
\hline 0.003617399 & -0.081265056 & 0.911524404 \\
\hline 0.042331176 & -0.887787865 & 0.116666958 \\
\hline 0.032812416 & 0.1798536 & 0.820612819 \\
\hline 0.035055714 & 0.124164769 & 0.83947853 \\
\hline 0.003195338 & -0.229052491 & 0.504847906 \\
\hline 0.035347485 & -0.060938774 & 0.925344008 \\
\hline 0.007536899 & -0.958030843 & 0.325413539 \\
\hline 0.000797786 & -0.251777072 & 0.876023417 \\
\hline 0.019045055 & -0.106859353 & 0.931131433 \\
\hline 0.005406086 & 0.420496097 & 0.386286082 \\
\hline 0.032589815 & -0.634757534 & 0.084478031 \\
\hline 0.004495245 & 0.267576995 & 0.842345222 \\
\hline 0.019707684 & -0.091015065 & 0.850282216 \\
\hline 0.046812846 & -0.568231975 & 0.588960812 \\
\hline 0.045460992 & -0.031342498 & 0.97116895 \\
\hline 0.045460992 & -0.658086795 & 0.482355396 \\
\hline 0.031561579 & -0.369802893 & 0.43031213 \\
\hline 0.011921076 & 0.29336219 & 0.286725759 \\
\hline 0.012170708 & 0.594873029 & 0.540951479 \\
\hline 0.01297431 & -0.485113822 & 0.482069252 \\
\hline 0.022845522 & -0.118484724 & 0.858829701 \\
\hline 0.031178225 & -0.327333102 & 0.250189628 \\
\hline 0.007124108 & 0.457608806 & 0.53705357 \\
\hline 0.04749154 & -0.032075555 & 0.973007929 \\
\hline 0.025807202 & 0.476032164 & 0.361728506 \\
\hline 0.012758369 & 1.016629561 & 0.084086573 \\
\hline 0.03415496 & -0.196870488 & 0.778859269 \\
\hline 0.038841625 & -0.501864997 & 0.523701005 \\
\hline 0.023768374 & -0.375630633 & 0.611095514 \\
\hline 0.002802275 & 0.224633837 & 0.721293011 \\
\hline 0.019104179 & -0.22046459 & 0.382713303 \\
\hline
\end{tabular}




$\begin{array}{rrr}0.044338313 & -0.573988076 & 0.478984484 \\ 0.040192545 & 0.052799756 & 0.947334503 \\ 0.044323514 & 0.035927247 & 0.973756367 \\ 0.011374659 & -0.376639788 & 0.686877497 \\ 0.010016526 & 0.037337733 & 0.975104936 \\ 0.019623357 & -0.019798497 & 0.976504324 \\ 0.011549849 & -0.08962169 & 0.878705558 \\ 0.020703872 & 0.34278695 & 0.14119703 \\ 0.007637052 & -0.11906947 & 0.822282608 \\ 0.017904759 & -0.328768607 & 0.740603358 \\ 0.034189408 & -0.147430423 & 0.926867442 \\ 0.006053019 & -0.43180461 & 0.06239882 \\ 0.025700496 & 0.107948939 & 0.893594985 \\ 0.014123041 & 0.172159431 & 0.915313614 \\ 0.04136958 & 0.468783765 & 0.068280016 \\ 0.049928301 & 0.687589264 & 0.464792247 \\ 0.024400901 & 0.269695413 & 0.588960812 \\ 0.013472871 & 0.988885315 & 0.446051422 \\ 0.046805456 & 0.293560558 & 0.28906354 \\ 0.032215926 & -0.112124245 & 0.783414487 \\ 0.019807083 & 0.480621118 & 0.086088543 \\ 0.026179106 & -0.45390268 & 0.134324512\end{array}$




\begin{tabular}{|c|c|c|c|c|}
\hline ProbeName & GeneSymbol & Description & logFC & AveExpr \\
\hline A_51_P259296 & Lpl & Mus musculus lipopre & -1.961023445 & 10.53863681 \\
\hline A_55_P1978465 & H2-Q5 & histocompatibility 2 Mus musculus histoci & -1.663988464 & 9.535892008 \\
\hline A_55_P2096867 & Gap43 & growth associated $\mathrm{p}$ Mus musculus growtl & -1.608736505 & 10.06212415 \\
\hline A_51_P292357 & Rps3a1 & ribosomal protein S: Mus musculus riboso & -1.46036708 & 14.50307495 \\
\hline A_51_P382970 & Itga9 & integrin alpha $9 \quad$ Mus musculus integri & -1.351976919 & 8.757361984 \\
\hline A_55_P2116650 & & 0 Mus musculus lung $R$ & -1.10377111 & 8.828398051 \\
\hline A_55_P1960735 & Gdf15 & growth differentiati Mus musculus growtl & -1.088687615 & 10.28319672 \\
\hline _55_P2106459 & Zfhx3 & zinc finger homeobc Mus musculus zinc fir & -1.010052712 & 8.340668201 \\
\hline A_51_P369803 & Psmb9 & proteasome (prosor Mus musculus protea & -0.963065252 & 10.67436193 \\
\hline A_55_P2001628 & Rps4l & ribosomal protein S، Mus musculus riboso & -0.939220016 & 12.10141308 \\
\hline A_51_P464308 & Gnb4 & guanine nucleotide Mus musculus guanir & -0.9391572 & 5817 \\
\hline A_55_P2052834 & Lst1 & leukocyte specific tr Mus musculus leukoc & -0.935420349 & 9.52643648 \\
\hline A_52_P463977 & Tmem140 & transmembrane pro Mus musculus transm & -0.919020928 & 9.96346538 \\
\hline A_55_P2163774 & Crip1 & cysteine-rich proteir Mus musculus cysteir & -0.915395828 & 12.97283366 \\
\hline A_51_P191782 & Olfml3 & olfactomedin-like 3 Mus musculus olfactc & -0.914211713 & 8.602884528 \\
\hline A_51_P135340 & Panx1 & pannexin 1 Mus musculus panne & -0.89971548 & 10.51056148 \\
\hline A_55_P2000973 & Syt15 & synaptotagmin XV Mus musculus synapt & -0.899192384 & 6.884 \\
\hline A_55_P2004960 & 9130017K11Rik & RIKEN cDNA 913001 Mus musculus 10, 11 & -0.852685098 & 7.532819994 \\
\hline A_52_P489295 & Adamts1 & a disintegrin-like anı Mus musculus a disin & -0.827379384 & 12.74595804 \\
\hline A_66_P112024 & & $0 \quad 0$ predicted gene 6816 & -0.70174101 & 11.68203967 \\
\hline A_51_P414126 & Rab19 & RAB19, member RA: Mus musculus RAB19 & -0.609561157 & 8.039804732 \\
\hline A_51_P455807 & Ehd4 & EH-domain containi Mus musculus EH-doI & -0.606539442 & 11.96303719 \\
\hline A_55_P2035951 & Haus8 & 4HAUS augmin-like Mus musculus 4HAUS & -0.604556892 & 12.67961674 \\
\hline A_55_P2121846 & & $0 \quad 0$ ribosomal protein S3, & -0.593846476 & 16.01148154 \\
\hline A_52_P593037 & Acsl5 & acyl-CoA synthetase Mus musculus acyl-Cr & -0.535221942 & 9.966696844 \\
\hline A_52_P472302 & Fxyd6 & FXYD domain-conta Mus musculus FXYD c & -0.531319508 & 15.21101819 \\
\hline A_51_P469968 & $\mathrm{H} 2-\mathrm{M} 3$ & histocompatibility 2 Mus musculus histoce & -0.519228813 & 8.643048954 \\
\hline A_51_P105520 & Nomo1 & nodal modulator 1 Mus musculus nodal & -0.452860024 & 13.6108246 \\
\hline A_55_P2079009 & Slco $2 b 1$ & solute carrier organ Mus musculus solute & -0.433713878 & 6.892808091 \\
\hline A_55_P1992160 & Mbp & myelin basic proteir Mus musculus myelin & -0.413993257 & 6.478592337 \\
\hline A_55_P2056186 & Siva1 & SIVA1, apoptosis-inc Mus musculus SIVA1, & -0.380111236 & 12.3637751 \\
\hline A_55_P2058953 & Rpl13a & ribosomal protein L: Mus musculus riboso & -0.330979256 & 15.77584397 \\
\hline A_55_P2041893 & Gm6404 & predicted gene 640 ¿ PREDICTED: Mus mus & -0.31280088 & 15.50642516 \\
\hline A_55_P2071326 & Rpl36 & ribosomal protein L: Mus musculus riboso & -0.285050556 & 15.50237923 \\
\hline
\end{tabular}




\begin{tabular}{rrr}
\multicolumn{1}{r}{ adj.P.Val } & Orre-Log_FC & Orre-adj.P.Val \\
0.002138263 & 2.300310172 & 0.020588553 \\
0.005659542 & 1.095400427 & 0.010853939 \\
0.002286646 & 1.534549449 & 0.020310107 \\
$1.30 \mathrm{E}-07$ & 1.159248599 & 0.022479083 \\
$9.24 \mathrm{E}-09$ & 1.432290312 & 0.030984266 \\
0.002824029 & 1.103008775 & 0.035505379 \\
0.00234358 & 1.475890823 & 0.029959263 \\
$6.68 \mathrm{E}-05$ & 1.571058738 & 0.04406641 \\
0.021316806 & 1.195568717 & 0.009768585 \\
0.000466592 & 0.7724143 & 0.022479083 \\
0.003570734 & 1.462830423 & 0.043323509 \\
0.008269886 & 0.855486614 & 0.014706557 \\
0.006768385 & 1.194912543 & 0.01368295 \\
0.013328699 & 1.479869423 & 0.020679583 \\
0.005752664 & 1.314593295 & 0.015213125 \\
0.024043042 & 0.703522655 & 0.011848366 \\
$7.61 \mathrm{E}-05$ & 1.206623188 & 0.042870119 \\
0.002774688 & 0.863387317 & 0.010153149 \\
0.002352939 & 1.18883491 & 0.028313328 \\
0.015404752 & 0.843857777 & 0.009585061 \\
0.007993662 & 0.975357457 & 0.021980244 \\
0.032697401 & 1.880255118 & 0.001780435 \\
0.011775353 & 0.850035903 & 0.023924257 \\
0.01463281 & 1.09546824 & 0.001887175 \\
0.011511499 & 1.283903087 & 0.006122076 \\
0.04119468 & 1.031697808 & 0.026852476 \\
0.008188084 & 0.786736671 & 0.027384624 \\
0.017975634 & 0.690060618 & 0.005963268 \\
0.01463281 & 1.097696112 & 0.023980247 \\
0.00429445 & 1.146770973 & 0.004021838 \\
0.010471067 & 0.943997477 & 0.013463823 \\
0.008188084 & 0.85748709 & 0.002800163 \\
0.028012031 & 0.758588 & 0.015596586 \\
0.001807237 & 0.72597553 & 0.008348755
\end{tabular}




\begin{tabular}{|c|c|c|c|c|}
\hline Category & Term & Count & PValue & Genes \\
\hline JP_KEYWORDS & Phosphoprotein & 282 & $1.62 \mathrm{E}-06$ & RAB3GAP2 \\
\hline OTERM_CC_DIRECT & GO:0005737 cytoplasm & 248 & $1.61 \mathrm{E}-04$ & RAB3GAP2 \\
\hline JP_KEYWORDS & Alternative splicing & 203 & 7.76E-09 & RAB3GAP2 \\
\hline UP_SEQ_FEATURE & splice variant & 189 & 1.17E-04 & RAB3GAP2 \\
\hline JP_KEYWORDS & Cytoplasm & 178 & $3.13 E-06$ & RAB3GAP2 \\
\hline SOTERM_MF_DIRECT & GO:0000166 nucleotide bindi & 81 & 0.006219 & GNA14, NA \\
\hline UP_KEYWORDS & Transport & 80 & 0.001058 & KCNC1, SLC \\
\hline UP_KEYWORDS & Nucleotide-binding & 78 & $2.53 \mathrm{E}-04$ & GNA14, NA \\
\hline GOTERM_BP_DIRECT & GO:0006810 transport & 78 & 0.002528 & KCNC1, SLC \\
\hline UP_KEYWORDS & Transferase & 74 & $3.19 \mathrm{E}-04$ & NDUFAF7, : \\
\hline GOTERM_MF_DIRECT & GO:0016740 transferase acti & 67 & 0.002022 & STK38, DPF \\
\hline UP_KEYWORDS & ATP-bin & 59 & 0.002958 & , NAF \\
\hline UP_KEYWORDS & Mitochondrion & 51 & $6.38 \mathrm{E}-04$ & NDUFAF7, \\
\hline GOTERM_CC_DIRECT & GO:0042995 cell projection & 39 & 7.83E-04 & LZTS1, SSH \\
\hline JP_KEYWORDS & Cell projection & 37 & 4.93E-04 & LZTS1, CRIF \\
\hline GOTERM_CC_DIRECT & GO:0048471 perinuclear regiı & 35 & 0.005406 & SNCG, YWr \\
\hline GOTERM_BP_DIRECT & GO:0006915 apoptotic proce: & 30 & 0.00565 & FGFR2, BID \\
\hline UP_KEYWORDS & Magnesium & 27 & 0.00652 & GNA14, STI \\
\hline INTERPRO & IPR001909:Krueppel-associat $\epsilon$ & 23 & 0.001542 & ZFP14, ZFP \\
\hline SMART & SM00349:KRAB & 22 & 0.007798 & 3 ZFP14, ZFP: \\
\hline OTERM_CC_DIRECT & GO:0005929 cilium & 18 & 0.00273 & RSG1, RAB \\
\hline UP_KEYWORDS & Polymorphism & 17 & 4.83E-04 & POLK, NLRF \\
\hline UP_KEYWORDS & Microtubule & 17 & 0.005302 & MAP1A, M \\
\hline UP_KEYWORDS & Cilium & 14 & 0.004355 & RSG1, RAB \\
\hline GOTERM_CC_DIRECT & GO:0043197 dendritic spine & 13 & 0.002169 & ANKS1B, KC \\
\hline GOTERM_BP_DIRECT & GO:0006914 autophagy & 12 & 0.004471 & LLEC16A, C \\
\hline UP_KEYWORDS & Nucleotidyltransferase & 8 & 0.006925 & NMNAT3, 1 \\
\hline GOTERM_BP_DIRECT & GO:0010918 positive regulati & 4 & 0.001443 & BID, TMEM \\
\hline INTERPRO & IPR026074:Microtubule associ & 3 & 0.00251 & MAP1S, M/ \\
\hline
\end{tabular}




\section{Fold Enrichment $\quad$ FDR $<10 \%$}

$\begin{array}{rr}1.258874304 & 0.002189798 \\ 1.209474637 & 0.22835511 \\ 1.444362564 & 1.05 \mathrm{E}-05 \\ 1.26870976 & 0.193801331 \\ 1.374326461 & 0.004233428 \\ 1.329545455 & 9.015407645 \\ 1.430952067 & 1.421628831 \\ 1.512105977 & 0.342463426 \\ 1.389752615 & 4.319319894 \\ 1.521294975 & 0.430194122 \\ 1.446407005 & 3.01979113 \\ 1.471883281 & 3.929882471 \\ 1.639085939 & 0.859654985 \\ 1.771367719 & 1.108038993 \\ 1.855620862 & 0.664640029 \\ 1.635633747 & 7.421532919 \\ 1.708589247 & 9.411594779 \\ 1.762151554 & 8.471744169 \\ 2.105922577 & 2.435743388 \\ 1.85439727 & 9.69841229 \\ 2.264975425 & 3.814035677 \\ 2.765794136 & 0.651727069 \\ 2.189587025 & 6.941046186 \\ 2.492366382 & 5.73475535 \\ 2.840571657 & 3.041949506 \\ 2.74336865 & 7.51980065 \\ 3.579263 & 8.974974792 \\ 16.23159785 & 2.487140808 \\ 34.15257048 & 3.9369346\end{array}$




$\begin{array}{ll}\text { Category } & \text { Term } \\ \text { UP_KEYWORDS } & \text { Membrane } \\ \text { GOTERM_CC_DIRECT } & \text { GO:0005730 nucleolus } \\ \text { UP_KEYWORDS } & \text { Ribosomal protein } \\ \text { GOTERM_MF_DIRECT } & \text { GO:0003735 structural constituent of ribosome } \\ \text { UP_KEYWORDS } & \text { Ribonucleoprotein }\end{array}$

Count PValue Genes

190.008025 9130017K1

60.007182 RPL13A, RF

40.002175 RPL13A, RF

40.00485 RPL13A, RF

40.006979 RPL13A, RF 


\section{Fold Enrichment $\quad$ FDR $<10 \%$}

$1.654266958 \quad 8.508138478$

$4.670308789 \quad 7.376940471$

$14.89655172 \quad 2.373863877$

$11.01388889 \quad 5.012444472$

$9.818181818 \quad 7.437309492$ 
Supplementary table 9. Comparison with young-versus-old mouse dataset

\begin{tabular}{|c|c|c|}
\hline Up-Up & Up-Zero & Up-Down \\
$\mathrm{UU}=12$ & $\mathrm{U} 0=516$ & $\mathrm{UD}=8$ \\
$\boldsymbol{E}[\mathrm{uu}]=7.2$ & $\boldsymbol{E}[\mathrm{u} 0]=523.4$ & $\boldsymbol{E}[\mathrm{ud}]=5.4$ \\
$p . \mathrm{v} .=0.0592$ & $p . v .=0.0308$ & $p . v .=0.1819$ \\
\hline Down-Down & Down-Zero & Down-Up \\
$\mathrm{DD}=8$ & $\mathrm{D} 0=439$ & $\mathrm{DU}=10$ \\
$\boldsymbol{E}[\mathrm{dd}]=4.6$ & $\boldsymbol{E}[\mathrm{d} 0]=446.3$ & $\boldsymbol{E}[\mathrm{du}]=6.1$ \\
$p . \mathrm{v} .=0.0972$ & $p . \mathrm{v} .=0.0248$ & $p . v .=0.0893$ \\
\hline
\end{tabular}


Supplementary table 10. Comparison with human AD dataset

\begin{tabular}{|c|c|c|}
\hline Up-Up & Up-Zero & Up-Down \\
$\mathrm{UU}=1$ & $\mathrm{U} 0=246$ & $\mathrm{UD}=3$ \\
$\boldsymbol{E}[\mathrm{uu}]=1.2$ & $\boldsymbol{E}[\mathrm{u} 0]=246.6$ & $\boldsymbol{E}[\mathrm{ud}]=2.2$ \\
$p . \mathrm{v} .=0.709$ & $p . v .=0.447$ & $p . v .=0.375$ \\
\hline Down-Down & Down-Zero & Down-Up \\
DD $=3$ & D0 $=242$ & DU $=0$ \\
$\boldsymbol{E}[\mathrm{dd}]=2.1$ & $\boldsymbol{E}[\mathrm{d} 0]=241.6$ & $\boldsymbol{E}[\mathrm{du}]=1.2$ \\
$p . \mathrm{v} .=0.363$ & $p . \mathrm{v} .=0.653$ & $p . \mathrm{v} .=1.000$ \\
\hline
\end{tabular}

